www.anatomy.org.tr

doi:10.2399/ana.14.001s

\title{
Abstracts for the 16th National Congress of Anatomy 11th-14th September, 2014, Malatya, Turkey
}

\section{Invited Lectures}

$$
\text { (I-1 - I-10) }
$$

\section{I-1}

\section{Current views on Turkish higher education \\ Bilgic S \\ Department of Anatomy, Faculty of Medicine, Ondokuz Mayıs University, Samsun, Turkey}

First university during republic period in Turkey was established on 1933. The numbers of universities increased to 7 in 1959,12 in 1973, 27 in 1982, 29 in 1987 (of one is private), 53 in 1992 (of 2 are private), 93 in 2006 ( 25 private), 177 in 2014 (73 private). Based on TUIK date, gross schooling rate is $74 \%$ and net schooling rate is $38.50 \%$ in higher education. However, based on the date of Higher Education Council, these rate are above \% 80 and $\% 40$ respectively with the number of students approaching to 5.5 million. Today, the number of teaching staff reached to 141,647 . Of this, $86.20 \%(122,116)$ employed by the state universities, $\% 13.44(19,188)$ are in private universities and $0.26 \%$ are in private vocational high schools. Total number of academic staff is 63,271 . Of this, $84.76 \%(53,630)$ employed in state universities. $31.43 \%$ of academic staff are professors, $19.97 \%(12,634)$ associate professors and $48.60 \%$ are assistant professor. Total number of students are $5,449,961$. Of this $93.38 \%(5,089,291)$ are studying in state universities, $6.44 \%(350,999)$ are private universities and $0.18 \%$ (9671) are in private vocational high schools.

\section{I-2}

Developing and adult human brain stem atlases and gene expressions

Şengül G

Department of Anatomy, Faculty of Medicine, Ege University, Bornova, Izmir, Turkey
Traditional mapping of the human brainstem, as of other anatomical structures, is based on information from Nissl and myelin-stained sections, and information from connections and physiology. Using chemical markers for marking has started with acetylcholinesterase staining from 1982 onwards, continuing with tyrosine hydroxylase staining for monoamines, and antibody markers (i.e. calbindin, parvalbumin). Using gene expression for defining anatomical structures is a new concept, after recent developments in molecular biology and biotechnology. With in situ hybridization (ISH), it is possible to localize DNA and RNA within intact cells and tissues without destroying the structure. Anatomical organization of the human brainstem is a complex mixture of compact neuronal groups (nuclei) and dispersed cell areas with varying cytoarchitecture. There are 438 structures we have defined so far in the human brainstem (Paxinoset al., 2012). I have performed the analysis of the human brainstem for Allen Institute BRAINSPAN Atlas of the Developing Human Brain and Atlas of the Human Brain projects, and prepared reference atlases for the developing 21 post-conception weeks (pcw) human brainstem. As examples, calretiningene CALB2 was expressed in the solitary nucleus, dorsal paramedian nucleus, and cochlear nucleus, glutamate gene GRIK2 in the gracile and cuneate nuclei, neuropeptide Y gene NPY in the intermediate reticular nucleus, and somatostatingene SST in locus coeruleus and medial parabrachial nucleus. This presentation will show how expression of genes in the brainstem define anatomy of the numerous nuclei, and the digital reference atlases created for 15 and $21 \mathrm{pcw}$,and adult human brain.(www.alleninstitute.org)

Keywords: Atlas, brainstem, human 


\section{I-3} Corticospinal neurons: in health and in sickness, until
death shall separate us

Ulupinar $\mathrm{E}^{*}$,**

* Department of Anatomy, Faculty of Medicine, Eskisehir Osmangazi Universit, Eskisehir, Turkey; **Department of Health Sciences Institute, Interdisciplinary Neuroscience, Eskisehir Osmangazi University, Turkey

Neurodegenerative diseases comprise a group of pathologies characterized by progressive and irreversible loss of neurons in certain regions of the brain. The most striking symptom of these disorders is that progression process more specifically against certain types of neurons. In Amyotrophic Lateral Sclerosis (ALS), there was a selective loss of lower and upper motor neurons in the spinal cord, brain stem and motor cortex regions. Diversity observed in the degeneration patterns of neurons suggest that interactions between intrinsic factors and environmental influences play an important role in the etiopathogenesis of this disorder. In this talk, I will first discuss why these specific neuronal populations are more prone to injury among the millions of neurons in the cortex. Then, I will focus on the molecules and mechanisms causing degeneration of these neurons. And finally, our data from an animal model having a genetic mutation that causes the selective loss of corticospinal motor neurons will be presented.

\section{I-4}

Anatomical errors in anatomy atlases

Sinav A

Professor\&Rector, SANKO University, Gaziantep, Turkey

Throughout history, anatomical information has been conveyed visually from teacher to student. Even in our modern era with advanced imaging technologies, still, no one can imagine an anatomy curriculum without hand illustrated anatomy atlases. The anatomical illustrations in atlases are used as self study materials by students, as slides for lectures etc... Atlases are especially crucial in countries where cadavers are not readily available for dissection. In such cases, anatomical illustrations provide students the sole point of access to internal structure of the human body. Unfortunately, atlas illustrations sometimes misrepresent anatomical information. The explanations for such inaccuracies are numerous. First, some medical illustrators do not possess sufficient understanding of anatomical details. Second, illustrators may pay more attention to the artistic features of their illustrations than to their anatomic accuracy. Finally, and most importantly, medical illustrators often use existing illustrations as resources rather than drawing on observations from actual dissections. This practice propagates errors through generations. Anatomy education can be considered the foundation of medical sciences. Therefore, visual materials must be prepared with painstaking accuracy. Anatomists need to assume responsibility for collaborating illustrators, in order that the mistakes of history may be rectified.
I-5

The National Core Curriculum-2014

(Undergraduate Medical Education) and the use in the anatomy education

Songur A

Department of Anatomy, Faculty of Medicine, Afyon Kocatepe University, Afyonkarahisar, Turkey

Since 1980s the increase in the number of medical faculties led to diversification of education models and programs in different medical faculties which have diverse infrastructure and capabilities. This condition indicated the need for preparing a framework program in order to train doctors with basic knowledge and skills. When the National Core Curriculum (NCC), prepared with contributions from medical faculties, was accepted in the early 2000s, it was decided that NCC should be revised every two years. However it still remains unchanged today. Medical Deans Council (TipDEK) took the decision to prepare a new NCC in the meeting in December 2012. Core curriculum was restructured and NCC-2014 was prepared with the contribution of numerous medical faculties, departments, faculty members and participators in the one year period following April 2013. NCC-2014 was discussed and approved by Council of Universities on 12 May 2014 and by Council of Higher Education in general assembly meeting on 19 June 2104. Educational outcomes and competence based approach were taken into account during the preparation of NCC-2014. NCC-2014 is for especially medical faculties and also all institutions interested in medical education. It is targeted at providing a framework for undergraduate medical education, defining the basis of medical education in national scale and thus establishing a standard for the undergraduate educational practice in accordance with international medical education principles and approach. It is advised that anatomy lectures, which have an important place in pre-clinical curriculum, should be associated with core diseases/clinical problems or basic clinical skills stated in NCC-2014. We think that associating anatomy lectures with NCC-2014 and giving anatomy lectures based on clinical problems will attract more interest from students and will facilitate learning. Furthermore students will not have to waste time on detailed information which is not clinically relevant.

Key words: National core curriculum, medical faculty, undergraduate medical education, anatomy.

\section{I-6 \\ What do we know about the status of hosting infective agent of the cadavers which has been stored in differ- ent conditions? How do we protect ourselves?}

Kurtoğlu Z

Department of Anatomy, Faculty of Medicine, Mersin University, Mersin, Turkey

The aim of the presentation is to assess the awareness of the anatomists, students, clinicians and staffs about the possible 
infection from cadavers, and the habits on the prevention of infection. In the first part of presentation, the results of questionnaire that prepared under the guidance of Anatomy Department of Mersin University will be shared. The questionnaire has been applied to 45 academic staffs from the Department of Anatomy, 44 surgeon who participated in cadaver dissections actively, and medical students from six different universities of Turkey. The results showed that we are not aware of the risk of infection and insufficient to take measures regarding the protection. According to data, it is clear that all individuals coming into contact with cadavers (i.e. anatomist academics, surgeons working in cadaver, undergraduate students and staffs carrying and maintain the cadavers, etc.) need to improve their knowledge in regard to the risk of infection. The second part of presentation includes literature information about the durability status of main infective agents (Tuberculosis, Hepatitis B and C, HIV, Creutzfeldt-Jacob Disease (CJD) and other prions) at the wet or dry environment and at the storage conditions with formaldehyde in liquid or with freezing in -20 degree. Finally, to regulate the contact process with the cadavers that protected in different formats, in a healthy manner, it is proposed to create an algorithm, prepared based on scientific data and having applicability, and to promote the implementation of this algorithm in all the anatomy labs.

\section{I-7}

\section{The historical and cultural past of Malatya}

\section{Karagöz M}

Department of History, Faculty of Arts and Sciences, Inönü University, Malatya, Turkey

$$
\begin{aligned}
& \text { Aspuzu is a nightingale garden which is rich and sacred } \\
& \text { Aspuzu is a great land which is reminiscent of Heaven } \\
& \text { Niyazi-î̀ Misrî } \\
& \text { (Aspuzu is the anchient name of present Malatya) }
\end{aligned}
$$

History has cut the umbulical cord of some residential areas at the World geography. Those areas are the surroundings that have been built by human hands and given cultural identity. Those kinds of residential places are the environments that contribute to joint civilization. Malatya is a city like that. There has been approximately a 10,000-year residential layout around Malatya. Findings indicated that there has been a State Order or Govermental System as from mid-4,000 BC have been unearthed. Thus, It has the characteristic of being one of the the oldest civilisation areas of the World. Culture and civilizations that have been introduced as from the ancient times have contributed to the joint human civilization due to Malatya's geography and cultural wealth of human communities' living in Malatya during history . Cultural and civic wealth continues to present time. Adequacy of geographical facilities has kept social and economic life lively; thus, societies lived in history have left a rich historical heritage. Provided that begining before Aslantepe, at present in Malatya, it is possible to find evidences coming from the Hittites, Romans, Seljuks, Ottomans and finally, Modern Republic's natural and cultural heritage.
I-8

\section{The publication and evaluation problems experienced before and after the examination for associated professorship}

Şakul BU

Department of Anatomy, Faculty of Medicine, Istanbul Medipol University, Istanbul, Turkey

The examination for associated professorship in academic progress is one of the most important and hard examinations for a candidate in a lifetime. The academician's studies in which he/she has done, contributed, exerted or his/her name has been mentioned without an effort until that time is assessed by the jury members. Obtaining the board of ethics' approval, obtaining it when and by whose name, or whose names would be written in which order; unfortunately; are such problems not known sufficiently or even probably ignored at both scholar and assistant levels, but still keeping itselves actual. Because of this; getting the approval of the board of ethics should be considered after signing up a protocol contract, covering the issues of who will take part in the study, perform which duties to what degrees and even how will the name order be determined before beginning such a study. Besides; one should be delicate on the matters of article's originality and mentioning the sources of quotations. Also there is a big burden of work and responsibilities to the jury members when it comes to evaluating the publications. Judging through a righteous review would prevent aggrieving the candidate. Blaming the candidates by violation of ethics or even plagiarism without a precise review would mean at least a 6 months' time of delay. The publications should be read very thoroughly and especially very carefully if an accusation of plagiarism is being considered. In addition to this; it should never be forgotten that the candidate in question has been working as an assistant professor, teaching assistant or as a specialist for at least 4 years. So it would be inappropriate to treat the candidates as a newly graduate assistant. Again, it should be borne in mind that groundless accusations may lead to administrative and legal inquiries against the accusers.

Keywords: Examination for associated professorship, ethics, plagiarism

\section{1-9}

\section{Groundbreaking anatomical reflections in Michelangelo's and Leonardo da Vinci's masterpieces}

Durgun B

Department of Anatomy, Faculty of Medicine, Çukurova University, Adana, Turkey

Objectives: Here, it is presented a historical perspective on Michelangelo and Leonardo da Vinci, their fascination for human anatomy and its symbolic use in their art.

Methods: Review and analysis of the reflections of human anatomy in the works of two artists.

Results: During the Renaissance, a substantial number of art works as well as scientific and medical treaties were produced, leading to an increased number of studies in human anatomy. 
It is not an easy task classify or separate the Renaissance exponents by their occupation due to the fact that many of them practiced more than one activity in an excellent way, as in the case of the sculptor, architect, poet, painter and a master anatomist Michelangelo Bounarroti (1475 -1564); and the artist, scientist, inventor, visionary, philosopher and the greatest anatomist of all time, Leonardo da Vinci (1452-1519). Both Michelangelo and Leonardo performed numerous cadaver dissections to expand their perspective in sculpture and painting. Michelangelo he did not publish any detailed drawings of his cadaver dissections since he probably destroyed his numerous anatomic sketches and notes. Unlike Michelangelo, Leonardo documented his detailed understanding of anatomy in numerous illustrations.

Conclusion: While in Florence during a continuous period of at least two years (1503-1505), Michelangelo came in contact with Leonardo repeatedly. Two genius men embodied the ideal of a continuing dialogue between art and science. They also reflected their profound anatomical knowledge on their masterpieces as realistic and symbolic manner. Some of them are Leonardo's "Virgin of the Rocks", "Mona Lisa", "Last Supper" and "Vitruvian Man"; and Michelangelo's "Creation of Adam", "Separation of Light and Darkness" and "David". The possible presence of the representation of anatomical structures in their masterpieces may be interesting, but the interpretation will inevitably be hypothetical.

Keywords: Michelangelo, Leonardo da Vinci, Anatomy, Renaissance and Anatomy

\section{I-10}

Functional and morphological approach to hippocampus in rat-pup model of hypoxic-ischemic encephalopathy

Adigüzel E

Department of Anatomy, Faculty of Medicine, Pamukkale University, Denizli, Turkey
Behavioral tests are used for evaluation of hippocampal function. Only histological methods are used to evaluate hippocampal structures. In this study hippocampal structures and function were evaluated in rat-pup model of hypoxic-ischemic encephalopathy. Four groups of rat-pups were used in the study; the first and second groups had been treated with subcutaneous $50 \mathrm{mg} / \mathrm{kg} /$ day grape-seed-extract from tenth day to the fifteenth day postnatally. The modified Levin model of hypoxic-ischemicencephalopathy was applied to the first and fourth groups in postnatal twelfth day. The third group was shameoperated group. Water maze and eight arm maze tests were applied ten weeks later subsequently. The rats were sacrificed, and serial sections of the brains were obtained. Parvalbumin, Calretinin, Neu Nimmunostaining were done, and H-SCORE method was used for evaluation. Total pyramidal neuron number was estimated in hippocampal pyramidal neuron layer by using stereological methods. The first group had lower performance than the other groups in water maze test. The performance of the fourth group was the lowest in eight arm maze $(\mathrm{p}<0.05)$. The third group had higher brain weight than those of the others. There was no statistically significant difference among the groups regarding the total pyramidal neuron number of hippocampus. H-SCORE of Parvalbumin + pyramidal neuron was higher and NeuN + pyramidal neurons was lower in the first group ( $\mathrm{p}<0,05$ for two parameters). We found that, subcutaneous injection of rape seed extract had not statisticallysignificant effect on total pyramidal neuron number, but it caused high number of Parvalbumin+, low number of $\mathrm{NeuN}+$ hippocampal pyramidal neuron in rat-pup model of hypoxic-ischemic encephalopathy. Differences between behavioral tests results indicated that the method preference is important in functional brain study.

This study was supported by TÜBİTAK (108S157) and Pamukkale Üniversitesi BAP (2008TPF005)

Keywords: Rat, pyramidal neuron, hippocampus, learning, memory 


\title{
Oral Presentations
}

\author{
(0-1 - 0-34)
}

\section{0-1}

\section{Investigation of branching variations of portal vein with multidetector computed tomography}

Ulusoy $M^{*}$, Koplay $M^{* *}$, Bolatlı $G^{*}$, Acar $M^{* * *}$, Zararsı I I*

*Department of Anatomy, Faculty of Medicine, Mevlana University, Konya, Turkey; **Department of Radiology, Faculty of Medicine, Selçuk University, Konya, Turkey; ${ }^{* *}$ Department of Physiotherapy and Rehabilitation, Vocational School of Health Services, Mevlana University, Konya, Turkey

Objectives: The aim of this study was to determine the types, prevalence and clinical implications of branching variations of portal vein (PV) in liver using multi detector computed tomography (MDCT).

Methods: Tomography images of 374 patients (172 male, 202 female) whose routine abdominal MDCT imaging were performed and without any liver pathology were evaluated. Frequency and types of PV variations were determined.

Results: Normal intrahepatic branching PV (139 males, 165 females) was identified as Type I. Variations of PV other than Type I were observed in 33 male and 37 female patients. Trifurcation of right and left PV branching from the main root of PV was identified as Type II (5.9\%). This variation was determined in 9 male $(5.2 \%)$ and 13 female (6.4\%) patients. In Type III branching variation (5.1\%), the right posterior PV was branching as a separate branch from the main PV. Type III variation was observed in 15 male $(8.7 \%)$ and 4 female (1.9\%) patients. Branching into three veins of right $\mathrm{PV}$ was assigned as Type IV (4\%). Type IV variant was observed in 5 male $(2.9 \%)$ and 10 female $(4.9 \%)$. Type V $(2.9 \%)$ variation was presenting as laying of the left PV to the opposite direction after deriving from the main PV. This variation was determined in 4 male $(2.3 \%)$ and 7 female $(3.5 \%)$ patients.

Conclusion: We have determined in our study that branching variations of $\mathrm{PV}$ were frequently seen. We can suggest that being aware of the variations of $\mathrm{PV}$ will decrease the probable risks in invasive interventions, transplantation and surgical procedures of the liver.

Keywords: Portal vein, Branching variations, MDCT

\section{0-2}

The effect of Ankaferd Blood Stopper as a drug on human umbilical vein endothelial cell culture (HUVEC)

Nisari $M^{*}$, Ulger $\mathrm{H}^{*}$, Torun $\mathrm{Y}^{* *}$, Özdemir $\mathrm{MA}^{* * *}$, Kaya $\mathrm{E}^{* * * *}$ *Department of Anatomy, Erciyes University School of Medicine, Kayseri, Turkey; ${ }^{*}$ Education and Research Hospital, Kayseri, Turkey; $* * *$ Department of Health of Child and IIIness, Erciyes University Faculty of Medicine, Kayseri, Turkey; ${ }^{* * *}$ Department of Microbiology, Faculty of Medicine, Erciyes University Kayseri, Turkey
Ankaferd BloodStopper (ABS) is an herbal extract which has been used historically as a haemostatic agent in traditional Turkish medicine. ABS comprises of standardized mixture of herbs $T$. vulgaris, G. glabra, V. vinifera, A. officinarum and U. dioica. Basic effect mechanism of ABS is the formation of an encapsulated protein web which represents the focus points for the vital erythrocyte masses. The haemostatic effects have been demonstrated by in vitro and in vivo studies. The aim of this study was to investigate the in vitro effects of the different times and doses of ABS on in vitro endothelial cell proliferation. Human umbilical cord obtained at Caesarean sections from Erciyes University hospital. After washing PBS, the cord vein lumen was filled with PBS containing $10 \mathrm{mg} / \mathrm{ml}$ collagenase and incubated 10 minute at $37^{\circ} \mathrm{C}$. The contents of the vein were gently flushed out with an equal volume of $30 \mathrm{ml}$ medium collected in a conical centrifuge tube then centrifugated at $1000 \mathrm{rpm}$ for 10 minute yielded a small white pellet which was resuspended in culture medium. The cells were plated in $2 \mathrm{ml}$ of medium at T-25 plastic flasks. The endothelial cells were passaged when confluent density was approached. After obtaining enough cells, the cells were counted by Thoma slide. Then cells were divided into control and five experimental groups. $5 \%$, $\% 25$ ve $\% 50$ ABS were added to medium of each experimental group $(n=16)$. After 48 hours culture period, the effects of ABS were determined by cell counting. It was microscopically observed that cells arised from the surface and adhered to each other after the ABS application to the HUVECs. Also, after 24 hours cells returned the normal growth and physiology. After 48 hours culture period, the effects of ABS were determined by cell counting. While the mean cell number of control group and experiment groups were $5.68 \times 10^{4}( \pm 1.7)$, $6.56 \times 10^{4}( \pm 1.09) 7.12 \times 10^{4}( \pm 1.14), 7.43 \times 10^{4}( \pm 0.89)$ respectively. There was a reduction on cell number in experimental groups when compared to the control. This reduction was statistically significant $(\mathrm{p}<0.05)$. In this study, we observed that Ankaferd has dual diverse dynamic reversible actions depend on dose and concentration in the model of HUVEC. ABS might have a role on numerous cellular mechanisms as well as its hemostatic actions.

Key words: ABS, HUVEC, umbilical cord

\section{0-3}

Determination of the bone and cartilage fields during forelimb development of rat using double staining and calculation of bone/cartilage ratios by stereology

Öztürk $M^{*}$, Unur E*, Acer $N^{*}$, Ertekin T*, Çınar Ş**, Meker $\mathrm{M}^{*}$, Tahta $\mathrm{Y}^{*}$

*Department of Anatomy, Faculty of Medicine, Erciyes University, Kayseri, Turkey; ${ }^{* \star}$ First and Emergency Aid, Ivrindi Health Services Vocational School, Balıkesir University, Balıkesir, Turkey 
This study aimed to calculate bone and cartilage ratios of forelimb belonging to newborn rats and fetuses in this study. In our study, fetuses and newborns are obtained from 21 pregnant rats. 7 groups are established and 3 of them were prenatal groups (16th, 18th and 20th days of pregnancy) and 4 of them were postnatal groups (birthday, 3rd, 7th and 12th days after birth). Fetuses and newborns were stained using double stained method. Times of primer and secondary ossification centers were observed in stained forelimb under stereomicroscope. Photos of bones were taken. Bone and cartilage surface ratios were calculated by using ImageJ program on these photos. In our study, primer ossification centers were observed in clavicula, scapula and humerus in 18th day of pregnancy and in radius and ulna on the 20th day of pregnancy. Secondary ossification centers were observed in scapula and humerus on the birthday and in radius and ulna on the 7 th day after birth. In 18th days fetus group that we used in our study, bone-cartilage ratio was observed \%43 in clavicula, \%20 in scapula, $\% 15$ in humerus but ulna and radius still remained cartilage. In 12 days newborn group, bone-cartilage ratio reached \%100 in clavicula, \%76 in scapula, \%69 in humerus, \%71 in radius and $\% 75$ in ulna. We hope that determination of bone and cartilage fields belonging to rat fore limb and development time of primer and secondary ossification centers will be a resource for future developmental and teratological studies.

This study was supported by Erciyes University Scientific Research Projects Unit TSY-11-3723

Keywords: Rat, bones, double staining, ossification, forelimb, stereology

\section{0-4}

\section{Anatomic and radiologic re-assessment of bile} duct length

$\underline{\text { Kocabiyik N*}}$, Imre $N^{*}$, Guven I**, Gulsen $M^{* * *}$, Yildiz $S^{*}$, Unlü $\mathrm{A}^{* * * *}$, Yazar $\mathrm{F}^{*}$

*Department of Anatomy, Gulhane Military Medical Faculty, Ankara, Turkey; ${ }^{*}$ Department of Radiology, Gulhane Military Medical Faculty, Ankara, Turkey; ***Department of Gastroenterology, Gulhane Military Medical Faculty, Ankara, Turkey; ****Department of General Surgery, Gulhane Military Medical Faculty

Despite numerous data relating to the anatomy of bile duct, its reported dimensions are showing differences. Accordingly, dimensions of bile duct was elaborated using different samples by prospectively performed cadaveric examinations and retrospective assessment of recorded MRCP (magnetic resonance cholangiopancreatography) and ERCP (endoscopic retrograde cholangiopancreatography) images obtained from the hospital database. The study involved 25 adult cadavers fixated in $10 \%$ formaldehyde and 100 MRCP and ERCP images. Extrahepatic bile ducts were exposed in the visceral aspect of cadaveric liver. The length of ducts between the right and left hepatic duct bifurcation to hepatopancreatic ampulla and bile duct (from cystic junction to hepatopancreatic ampulla) were respectively measured using digital calipers. Besides, the cystic duct and common bile duct length were also measured. The archived
MRCP images were measured retrospectively. Bile duct length was measured using digital calipers on MIP (Maximum Intensity Projection) images at the Radiology Department Imaging Workstation. The mean length of cadaveric bile duct, cystic duct and common bile duct was $60 \pm 10.2 \mathrm{~mm}, 21 \pm 5.95$ $\mathrm{mm}$ and $23.5 \pm 9.71 \mathrm{~mm}$, respectively. The mean MRCP and ERCP length of choledoc duct was $58 \pm 9.95 \mathrm{~mm}$ and $59 \pm 9.85$ $\mathrm{mm}$, respectively. In the literature, the reported length of bile duct varies between $50-150 \mathrm{~mm}$. In contrast to these findings, the duct length seems much shorter in our study. We think that the updated data derived from this study is likely to provide a better guide to clinicians for gall bladder, pancreas and duodenum interventions and contribute to the literature.

Keywords: Common bile duct, cystic duct, common hepatic duct

\section{0-5}

Estimation of the volume of the tympanic cavity, cochlea and spiral ganglion neurons using stereological methods in the human newborn cadavers

Acer $\mathrm{N}^{*}$, Kesici $\mathrm{H}^{* *}$, Unur E*, Sönmez $\mathrm{MF}^{* * *}$, Ertekin T*, Arslan $A^{*}$, Bilgen $M^{* * * *}$, Çoşkun $A^{* * * * *}$, Erkan $M^{* * * * * *}$

*Department of Anatomy, Faculty of Medicine, Erciyes University, Kayseri, Turkey; ${ }^{*}$ Department of Histology-Embriyology, Faculty of Medicine, Gaziosmanpaşa University, Tokat, Turkey; ***Department of Histology-Embriyology, Faculty of Medicine, Erciyes University, Kayseri, Turkey; $* * * *$ Department of Biostatistic, Faculty of Medicine, Adnan Menderes University, Aydın, Turkey; $* * * *$ Department of Pediatric Radiology, Faculty of Medicine, Erciyes University, Kayseri, Turkey; $* * * * *$ Department of Ear Nose Throat, Faculty of Medicine, Erciyes University, Kayseri, Turkey

The aim of this study is to calculate the tympanic cavity (TC) and cochlea volume with manual tracing technique on computed tomography images of temporal bone with a software developed in house using MATLAB platform. Images with slice thickness of $0.25 \mathrm{~mm}$ were obtained from 5 newborn cadavers and analyzed independently by two expert researchers using the software and the results were compared. The borders of TC and cochlea were delineated manually and measurement of interested area was calculated on masked images. To confirm the agreement of the histological and computed tomography (CT) data were compared. Then, the area measurements from all sections were added for the calculation of total volume. Also, we estimated neuron number of spiral ganglion using optical fractitoner method. The neurons within the counting areas sampled were counted with the optical disector technique. The total number of the newborns spiral ganglion neurons was estimated by multiplying the number of neurons counted by the reciprocal of the aggregate sampling fraction. We found an average of $38715 \pm 1200$ neurons with a coefficient of variation of 0.19 . Dice similarity coefficient, Jaccard, sensitivity and specificity measures were used as a statistical validation metric to evaluate the performance of manual volume measurement. Good agreement was observed between MAT$\mathrm{LAB}$ and ImageJ on TC volume results. Validation results 
showed that the average Dice values being above 0.8 indicating the software which allows consistent and reliable volume measurements. In conclusion, these softwares are accurate and robust in TC volume, providing an objective tool for quantitative assessment related to tympanic membrane perforations. This approach is a great benefit for image-based analysis and planning in the field of computer-assisted surgery as well as volume calculation. This software can potentially be applied to measure other anatomical volumes from radiological images.

This study was supported by the Department of Scientific Research Projects of Erciyes University. (project number: TOA-11-3618).

Key words: Temporal bone, tympanic cavity, cochlea, volume, hacim, spiral ganglion, stereology

\section{0-6}

Anatomical investigation of insertion of tendo calcanei

\section{Gümüșalan $\mathrm{Y}^{*}$, Üzel $\mathrm{M}^{* *}$, Tuğtağ B*}

*Department of Anatomy, Faculty of Medicine, Fatih University, Istanbul, Turkey; **Department of Orthopaedics and Traumatology, Faculty of Medicine, Kahramanmaraş Sütçü Imam University, Kahramanmaraş, Turkey

Objectives: Achilles' tendon (tendo calcanei), in addition to being the most durable tendon of the body, is one of the structures experiencing pathologic cases such as tendinopathies as well as being ruptured most frequently. Understanding the distances of muscular fibres to the site of insertion of lateral and medial heads of gastrocnemius and soleus muscles forming the Achilles' tendon; site, form and angle of insertion of the tendon to the calcaneus is very important from the point of both understanding the biomechanical function of the tendon and decrease the risk of iatrogenic injury that may occur during the surgical treatment. We aimed to demonstrate the anatomical features of insertion of calcaneal tendon in this study.

Methods: Gastrocnemius and soleus muscles in the posterior compartment of leg were dissected from the origins to the insertions in 25 lower extremities amputated because of ischemic causes without any congenital structural anomaly. The insertional area, angle of insertion to the calcaneus, dimensions at the insertion and thinnest point of Achilles' tendon, as well as tendon lengths from the insertion to the medial and lateral heads were all measured.

Results: In 25 lower extremities dissected, average length of Achilles' tendon to the lateral head was $18,9 \mathrm{~cm}$, to the medial head was $18,6 \mathrm{~cm}$. Its dimensions were measured as $16 \times 7 \mathrm{~mm}$ at its thinnest point and $32 \times 7 \mathrm{~mm}$ at its insertion. A bursa located between calcaneus and tendo calcanei was determined having a shape of isosceles triangle and its margins were measured as 22-22-31 $\mathrm{mm}$. Distal to this triangle, Achilles' tendon was inserting to the calcaneus by expanding from medial to lateral. In addition, calcaneal tendon was not in the exact coronal plane while inserting into the calcaneus, and it inserted by making a lateralward angle of approximately $15^{\circ}$ with the coronal plane.

Conclusion: The insertion of Achilles' tendon which is known as 'Enthesis' organ was the study subject to many investigators.
Kager's fat pad and retrocalcaneal bursa in close contact with the tendon decrease the stress between the bone and soft tissue. It is thought that this situation is closely related with the form of insertion of the tendon to the calcaneus. In contrast to the definition in classical textbooks, Achilles' tendon inserts posterior to the calcaneus not directly at its middle point or top, but most fibers at medial to the midline and expanding laterally and to the plantar aspect of tuber calcanei.

Conclusion: In conclusion, we think that this reality is directly related with biomechanics of the foot, hence the burden on the tendon may slide medially especially during walking and affect the gate.

Keywords: Achilles' tendon, calcaneus, insertion

\section{0-7}

Opinions of university students on organ donation and whole body donation in anatomy education: Baskent University sample

Öktem $\mathrm{H}^{*}$, Özşahin E*, Yildirim RV**, Kürkçüoğlu A*, Yazici $\mathrm{C}^{* * *}$, Pelin $\mathrm{C}^{*}$

*Department of Anatomy, Faculy of Medicine, Baskent University, Ankara, Turkey; **Department of History of Medicine and Ethics, Faculy of Medicine, Baskent University, Ankara, Turkey; ${ }^{* * *}$ Department of Biostatistics, Faculy of Medicine, Baskent University, Ankara, Turkey

Objectives: Although artificial models, simulators reflecting the natural structure or computer based three dimensional images and even virtual cadavers are now being widely used in anatomy education, human cadaver dissection still keeps importance in medical curriculum. In addition their experiences in anatomy laboratory and the time spent for cadaver dissections makes the medical students get used to the notion of death. On the other hand so many people lose their lives due to multiple organ insufficiencies. It is well known that organ transplantation is their last chance to gain back their life quality. However both organ donation and whole body donation still keep on being a great problem. The aim of the present study is to evaluate the opinions of university students receiving training in the field of health on organ and whole body donation.

Methods: 643 subjects with a mean age of $20.96 \pm 7.56$ years participated to the study. All the subjects were the students of the faculties giving education in the field of health in Başkent University. The participants were requested to fill a questioner containing 20 three-point Likert scale questions.

Results: Although $71.5 \%$ of students said "yes" to the statement "I would like to be useful after death", 59.5\% of them do not want their body to be used as teaching or experimental material. $85.4 \%$ of the participants think cadaveric dissection aids the medical students to upgrade their knowledge on medical science. However $38.9 \%$ of them think medical students disrespect to the individual who donated his body to education during their practice. $78.1 \%$ of the students are willing for being organ donors. However of the ones who had accepted to donate their organs $73.3 \%$ refused to donate their bodies $(\mathrm{p}<0.001)$.

Keywords: Body donation, organ donation, medical education 


\section{0-8}

Neurotoxic effects of experimental epilepsy, lamotrigine and phenytoin on the brain tissue of newborn rats

Soysal H* , Doğan $Z^{* *}$, Eşrefoğlu $M^{* * *}$, Türköz $Y^{* * * *}$

*Department of Anatomy, Faculty of Medicine, Baskent University, Ankara, Turkey; **Department of Anatomy, Faculty of Medicine, Adıyaman University, Adıyaman, Turkey; ***Department of Embryology and Histology, Faculty of Medicine, Bezmialem

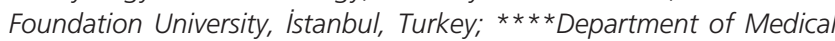
Biochemistry, Faculty of Medicine, Inönü University, Malatya, Turkey

Objectives: The aim of the study is to observe the postnatal effects of Lamotrigine (LTG) and Phenytoin (PHT) on the fetal brain tissues of the pregnant rats that the experimental epilepsy (EE) is practised on. Also it's aimed to determine the protective role of folic acid (FA).

Methods: In our study 27 rats were used. Pregnant rats divided into 10 groups in such away that Control $(\mathrm{n}=2), \mathrm{EE}(\mathrm{n}=3)$, PHT $(\mathrm{n}=2)$, PHT+EE $(\mathrm{n}=3), \mathrm{PHT}+\mathrm{FA}(\mathrm{n}=3), \mathrm{PHT}+\mathrm{FA}+\mathrm{EE}(\mathrm{n}=3)$, LTG $(n=3)$, LTG+EE $(n=2)$, LTG+FA $(n=2), L T G+F A+E E$ $(\mathrm{n}=4)$. During pregnancy we applied $2 \mathrm{mg} / \mathrm{kg}$ LTG to Lamotirgine groups, $25 \mathrm{mg} / \mathrm{kg}$ PHT to Phenytoin groups and $400 \mu \mathrm{g} / \mathrm{kg}$ FA to Folic acid groups every day. On the 13th day of the pregnancy rats were implemented by sterotaxic initiative. After birth on day 0th (70), 21th (75) and 38th (65) days of 210 newborn rats brain tissues were examined underlined light microscope.

Results: Layers of the cerebral cortex of 0, 21 and 38 day-old rats arised from pregnant rats which experimental epilepsy applied and lamotrigine used during pregnancy were observed perivascular edema, hemorrhage, cell infiltration, vacuolization. Phenytoin and folic acid were used in the postnatal group 0,21 and 38 day-old rats cellular properties of the layers of the cerebral cortex were same with control group.

Conclusion: Epileptic seizures and the use of lamotrigine during pregnancy was observed to cause pathological damage in the brain tissue throughout postnatal period. Phenytoin did not effectuate any tissue damage and the protective role of folic acid wasn't observed. As a result we believed that epileptic seizures and the use of lamotrigine during pregnancy can be possible to create in the brain tissue of offspring.

Keywords: Experimental epilepsy, phenytoin, folic acid, lamotrigine

\section{0-9}

\section{Evaluation of relation between the transvers foramina} and dominance of vertebral arteries

$\underline{\text { Karacan } \mathrm{K}^{*}}$, Karacan $\mathrm{A}^{* *}$, Acar B*** , Acar T***

*Department of Anatomy, Faculty of Medicine, Sakarya University, Sakarya, Turkey; **Department of Radiology, Sakarya University School of Medicine, Sakarya, Turkey; ${ }^{* * \star D}$ Department of Neurosurgery, Sakarya University School of Medicine, Sakarya, Turkey

The transvers foramina (TF) of cervical vertebra are known to transmit the vertebral arteries (VAs) and accompanying venous and sympathetic plexuses. The vertebral arteries (VAs) arise from the superoposterior aspect of the subclavian's first part, ascending through the foramina in all cervical transverse processes expect the seventh, to curve medially behind the lateral mass of the atlas and then ascending to enter the cranium via the foramen magnum. The diameters of VAs are usually unequal in size, the left being larger than right. Recent studies have suggested that inequality of VA blood flows may have certain hemodynamic effects on the vertebrobasilar system. Moreover, knowing the side of the dominant VA may be of utmost importance if VA is at risk during spine surgery. Therefore, we aimed to determine the relationship between the TF and Vas. Twenty six patients (15 male, 11 female) who were treated for cerebrovascular pathologies between January 2013 and June 2014 were included in the study. Ages varied between 28 and 60 years. Screening was performed by cervical computed angiography. Two measurement (maximum right-left and anterior-posterior diameter) were obtained for each transvers foramina at levels C3-6. We found that dominance of left VA in 16 cases (61.53\%). Moreover there was a strong correlation between TF and VA diameters. TF matched with the side of dominant VA in 21 of $26(80.76 \%)(\mathrm{p}<0.05)$. There was a harmony between the dominant VA and larger side of TF. Thus our findings demonstrate that the larger side of TF can be used reliably to predict the side of the dominant VA.

Keywords: Vertebral artery, transverse foramina, cervical vertebra

\section{0-10}

The nucleus of Darkschewitsch: three-dimensional reconstruction, photorealistic imaging, morphometric and volumetric evaluation

Terim Kapakin $\mathrm{KA}^{*}$, Kapakin $\mathrm{S}^{* *}$

*Department of Pathology, Faculty of Veterinary Medicine, Atatürk University, Erzurum, Turkey; **Department of Anatomy, Faculty of Medicine, Ataturk University, Erzurum, Turkey

The purpose of this study was to create computer-aided design (CAD) models of the nucleus of Darkschewitsch and evaluate it morphometrically and volumetrically. With the morphometrical evaluation of reconstructions of the nucleus of Darkschewitsch, on the right side, the nucleus of Darkschewitsch distances were $1.1917 \mathrm{~mm}$ vertically, $0.03595 \mathrm{~mm}$ laterally, and $0.06512 \mathrm{~mm}$ anteroposteriorly. On the left side, the nucleus of Darkschewitsch distances were $1.1918 \mathrm{~mm}$ vertically, 0.03600 $\mathrm{mm}$ laterally, and $0.06515 \mathrm{~mm}$ anteroposteriorly. The volumes of the right and left nucleus of Darkschewitsch of rat were $0.00069 \mathrm{~mm} 3$ and $0.00068 \mathrm{~mm} 3$ respectively. The surface areas of the right and left nucleus of Darkschewitsch were calculated $0.12250 \mathrm{~mm} 2$ and $0.12444 \mathrm{~mm} 2$ by using Surfdriver software. The mean distance between the right and the left nucleus of Darkschewitsch was $0.03676 \mathrm{~mm}$. Three-dimensional reconstruction of the nucleus of Darkschewitsch made the nucleus of Darkschewitsch to visualize, precieve and interpret and enabled to rehearse prior to surgical intervention.

Key words: Rat, the nucleus of darkschewitsch, three-dimensional (3D) model, photorealistic 


\section{0-11}

Face transplant questioned-experimental or scientific?

Öztürk L, Boduç E, Görür İ

Department of Anatomy, Faculty of Medicine, Ege University, Izmir, Turkey

Objectives: Scientific knowledge is the knowledge which is obtain through scientific methods. Scientific method is based on mind,observation and experiment. The criterion of scientific knowledge is to be methodological. Scientific knowledge is systematic, consistent, and objective. it is also suitable to invite criticism. Unlike scientific knowledge than tecnical information is the scientific knowledge includes theoretical knowledge instead of application information. Technical knowledge is not always derived from scientific knowledge. İt is derived from social, commercial, political, ethic, aesthetic and psychic origin concerns. The purpose of science is to discover the rules of nature dominate. As well as to evaluate the systematic information obtained for considering for the benefit of the nature,human and humanity. In the scientific thought,there is no order to create a new mindset by changing and forcing the rules of nature which is dominated. Scientific integrity predicts being moral, fair, sincere and having respect to labor during the search for truth in action. The scientist is not a science technican who applies known technical information. The scientist is a wise who fully internalizes moral philosophy. Presentation to the public of initiatives or work in scientific institutions is an another responsibility. The fulfillment of this responsibility as required is important for social credibility and prestige of the scientist. The responsibilit of the scientist is to follow the initiatives, the works and the publications which is located in the area of expertise. Also examine the idea or process advanced with a critical approach, and clarify the scientific response in case of any suspicion and deviation.

Methods: In seven (7) cadavers we removed scraping the soft tissue of face, sometimes partially and sometimes completely, including the periosteum according to the face transplant method of dealing with that is recorded on the articles with the scientific responsibility as mentioned above. During the exercises on cadaver, we also mastered about the details and importance of these interventions.

Results: We investigated face transplats performed given training is dominated by biomedicine in our country with in line with the three main theories. The face transplants performed to review three basic theories that we hold are; 1-Cell Theory (Pioneers:Matthias Schleiden,Theodar Schwann, Lorenz Oken,Rudolf Wirchow; All living things are composed of cells), 2-Heredity (gene and chromosome theory) (Pioneers: Theodor Boveri and Walter Sutton; The full information encoded in the features of living things are in cells.) 3-The theory of evolution (Pioneers:Charles Darwin and Alfred Russel Wallace; All living things have a common origin and are formed by long-term slow changes.)

Conclusion: Face transplant attempt, analyzing with the light of the theory of bio-medical's underlying and also given education in our country, is not scientific; it is experimental. Moreover, it will not only create biological problems on the experimental subjects of facial transplantation.

\section{0-12}

\section{Morphometric analysis of the orbit and orbital structures with three-dimensional multidetector computed tomography}

Turamanlar $\mathrm{O}^{*}$, Acar T**, Unlu E***, Gonul Y*, Demirtas I**, Songur A*

*Department of Anatomy, Faculty of Medicine, Afyon Kocatepe Universty, Afyonkarahisar, Turkey; ${ }^{* *}$ Department of Anatomy, Faculty of Medicine, Şifa University, Izmir, Turkey; ${ }^{* * * D e p a r t m e n t ~ o f ~ R a d i o l o g y, ~}$ Faculty of Medicine, Afyon Kocatepe University, Afyonkarahisar, Turkey

Anatomic characteristics of orbit and the soft tissue therein may exhibit differences. In this study, face, head and maxillofacial BT (160-slice MDBT) images of 100 people (58 males, 42 females) taken, were retrospectively evaluated. Comparisons were made between decades, gender, parameters and both orbit. In grading of decades, parametres "minimum distance between medial walls of orbit" and "left rectus inferior muscle" were found to be statistically meaningful. Statistically meaningful differences were found in comparison of minimum distance between medial walls of orbit parameter between 3rd and 6th decades, and left rectus inferior muscle parameter between 8 th decade and $2 \mathrm{nd}$, 3rd and 4th decades. In comparison of female and male groups in regard of parameters, the parameters "maximum distance between internal and external walls of right orbit", "maximum distance between internal and external walls of left orbit", "maximum distance between upper and lower walls of right orbit", "distance between right for.infraorbital and lower wall of orbit", "distance between left for.infraorbital and lower wall of orbit", "interzygomatic line length", "skull transvers diameter", "left rectus superior muscle", "right rectus lateralis muscle", "left rectus lateralis muscle" and "right optical nerve width" exhibited statistically meaningful differences between the groups. In comparison of parameters of right and left orbit, the parameters "maximum distance between internal and external walls of orbit" and "maximum distance between upper and lower walls of orbit" were found to be statistically meaningful. The relations between "skull transvers diameter" and "interzygomatic line length", "skull transvers diameter" and "maximum distance between internal and external walls of right orbit", "interzygomatic line length" and "maximum distance between internal and external walls of left orbit" and "maximum distance between internal and external walls of right orbit" were found to be statistically meaningful.

Keywords: CT, diameter, morphometry, optic nerve, orbit

\section{0-13}

\section{A detailed anatomic study of certain landmarks of the posterolateral corner of knee}

Elvan $\mathrm{O}^{*}$, Kurtoğlu $Z^{*}$, Aktekin $\mathrm{M}^{*}$

*Department of Anatomy, Faculty of Medicine, Mersin University, Mersin, Turkey

To reveal the morphologic properties of the structures which were important in the posterolateral corner surgeries was aimed. Morphometric properties of lateral collateral ligament (LCL) 
and popliteus tendon (PT) were focused on. Posterolateral corner of the knee joint was bilaterally dissected in 10 male cadaveric knees (aged between 45 and 93). The distances of NPC to Gerdy tubercle (GT), caput fibula (CF) and LCL were measured. The distance of inferior lateral genicular artery and veins (ILG) to CF and lateral epicondyle was evaluated. The width and thickness of superior and inferior parts and length of the LCL were measured. The relation of LCL with biceps femoris muscle tendon (BT) and PT considering synovial structures of this region was evaluated. Width and thickness of PT at upper and middle parts were measured. The shape of femoral attachment of PT, the positional relation of PT with LCL and joint capsule were identified. The statistical relation of PFL's morphometric outputs with LCL and PT was evaluated. In 7 cases, LCL overlapped the PT; in 12 cases femoral attachment of the PT surpassed the front side of femoral attachment of LCL (4.60 \pm 2.99 $\mathrm{mm})$. In 1 case, it stayed completely in front of LCL. In 12 cases, there was synovial sac around the inferior part of LCL. In 6 cases this sac covered the LCL superficially and deeply like vagina synovialis. In 8 cases it was found like a bursa superficially on the LCL. In the others, BT and LCL were merged with each other and then ended. In 3 cases, there was a synovial sac between the LCL and PT at the femoral side. The upper part of LCL was larger than the lower part $(\mathrm{p}=0.0001)$. There was no difference between upper and lower parts of LCL $(\mathrm{p}>0.05)$. No difference was found between the wideness of upper and middle parts of PT. The schematic model which was describing the positions of NPC and ILG and specifying the morphometric features of LCL and PT was designed. The model which was designed from the findings of this study may be used as a practical pathfinder in the posterolateral corner surgeries.

Keywords: posterolateral corner, lateral collateral ligament, popliteus tendon

\section{0-14}

The anatomy of the posterolateral sulcus of the spinal cord: its clinical significance

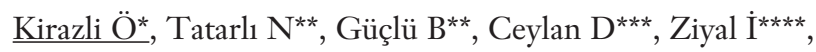
Keleş $\mathrm{E}^{* * * * *}$, Çavdar $\mathrm{S}^{* * * * * *}$

*Department of Anatomy, Faculty of Medicine, Marmara University, Istanbul, Turkey; **Department of Neurosurgery, Dr. Lutfi Kirdar Kartal Education and Research Hospital, Istanbul, Turkey; ***Department of Neurosurgery, Faculty of Medicine, Sakarya University, Sakarya, Turkey; $* * * *$ Institute of Neurological Sciences, Marmara University, Istanbul, Turkey; $* * * * *$ Department of Neurosurgery, Faculty of Medicine, Koc University, Istanbul, Turkey; $* * * * * *$ Department of Anatomy, Faculty of Medicine, Koç University, Istanbul, Turkey

Objectives: The posterolateral sulcus (PLS) of the spinal cord is an important land mark for the dorsal root entry zone (DREZ) operation and for the removal of the laterally located spinal intramedullary tumors. In order to achieve a successful surgery, detailed knowledge of the anatomy and the variation of the region is necessary.

Methods: Five formalin embalmed adult human cadavers (1 female and 4 males) were used. The number of posterior rootlets in each spinal level were identified and documented. The dorsal roots were examined under $10 \mathrm{X}$ microscope magnification. Transverse paraffin sections (10 İm) were cut and mounted on slides. To visualize white and the gray mater of the spinal cord slides were stained with luxol fast blue. The PLS and the dorsal horns were defined and measurements were made at X4, magnification, and photographed using a Nikon camera.

Results: Histological examinations of the PLS showed a single layer of pia which extended between the dorsal and the lateral columns. Any separations between the dorsal and the lateral columns that did not contain pia were not evaluated as sulcus. There was no arachnoid covering within the sulcus. The number of dorsal rootlets at cervical levels ranged between 2-10 and the mean number was $7.6 \pm 1.4$. The number of dorsal rootlets at thoracic level ranged between 3-9 and the mean number was $6.5 \pm 0.7$. The number of dorsal rootlets at lumbar level ranged between $4-8$ and the mean number was $6.1 \pm 0.4$. The location of the extension of the pia into the PLS showed variations. Length and width measurements of the dorsal horn showed that the average width was largest at lower cervical and least at lower thoracic, whereas the average length was largest at upper thoracic and smallest at lower lumbar.

Conclusion: Being aware of the variations of the PLS and the average number of rootlets at each spinal level can increase the success of regional surgery.

Keywords: Posterolateral sulcus, spinal cord, anatomy

\section{0-15}

\section{Augmented reality in anatomy education}

Kapakin S

Department of Anatomy, Faculty of Medicine, Ataturk University, Erzurum, Turkey

Augmented reality (AR) reaching to last user in the 2000s is a cutting edge technology that enables to combine the real world with virtual world. Augmented reality is a new application area which you can embed in real time three-dimensional (3D) objects generated by computer into real life videos. The aim of this study is to define the concept of augmented reality and examine potential uses in anatomy education. Three basic components of the augmented reality technology are the display, tracking system and mobile computer. The camera connected to computer captures the images in traditional way. When the camera detects the marker in the image, captures the information encoded in it and it sends this information to the computer. The computer decode information and projects $3 \mathrm{D}$ objects on the marker. It appears as if $3 \mathrm{D}$ objects were parts of the real world. The computer can pursue the size and movements of the $3 \mathrm{D}$ objects. The augmented reality makes lectures more attractive. It makes boring lectures funny. It makes difficult topics easier to learn. It provides easy access to course material which is difficult to obtain. It improves quality of education. Augmented reality enables a mobile technology user to interact with virtual world embedded within the physical world.

Keywords: Anatomy, augmented reality (AR), education 


\section{0-16}

Estimation of liver volume and surface area of the newborns' cadaveric liver using stereological methods

Avnioğlu $\mathrm{S}^{*}$, Unur $\mathrm{E}^{* *}$, Ülger $\mathrm{H}^{* *}$, Acer $\mathrm{N}^{* *}$, Çınar Ş***, Sağıroğlu $A^{* * * *}$

*Health School, Kahramanmaraş Sütçü Imam University, Kahramanmaraş, Turkey; **Department of Anatomy, Faculty of Medicine, Erciyes University, Kayseri, Turkey; ***First and Emergency Aid, Iurindi Vocational School of Health Service, Ballkesir University, Ballkesir,

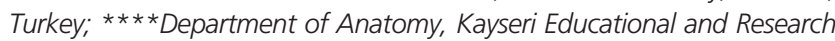
Hospital, Kayseri, Turkey

Many changes are observed in size and morphology of many diseases of the liver. The treatment follow up of these changes are important in diagnosis. It is possible to get an idea about the size of liver by the physical examination. However, the best results can be presented with imaging methods. In this study, dimensional measurements were made in five newborns' cadaveric livers in the laboratory of the Anatomy Department at Erciyes University, Medical Faculty with USG and calipers, and stereological measurements were also made on the images of MRI and the sliced livers. In this study, Cavalieri principle, a reliable method, was used to measure liver volume of the newborns with MR images. The volume which is calculated according to the principle of Archimedes is the actual volume of the liver and this value was accepted as the gold standard. We compared the estimated values obtained from ultrasound, MR images and slicing method with this measurement accepted as the gold standard. We calculated the mean liver volumes obtained with MRI, USG, slicing method and the gold standard to be $59.00 \pm 40.46 \mathrm{~cm}^{3}$, $115.86 \pm 102.51 \mathrm{~cm}^{3}, 85.88 \pm 68.22 \mathrm{~cm}^{3}, 70.00 \pm 49.96 \mathrm{~cm}^{3}$, respectively. We calculated the mean liver surface areas in 4 orientations, using cycloid probe. The results were $403.46 \pm 171.39 \mathrm{~cm} 2$, $266.81 \pm 113.83 \mathrm{~cm}^{2}, 265.76 \pm 179.76 \mathrm{~cm}^{2}, 186.82 \pm 76.77 \mathrm{~cm}^{2}$ slicing method, axial, coronal and sagittal planes, respectively. It was found from the results obtained from MRI slices that the volume values calculated in aksial plane in $1.6 \mathrm{~mm}$ slice thickness were the closest values to the gold standard. In conclusion, we are of the opinion with the present study that, we can contribute to the clinic on the size and morphology of liver disease associated with many serious clinical evaluation and planning of liver transplantation in the clinic.

Key words: Hepatometry, liver volume, surface area, stereology, cavalieri principle

\section{0-17}

Anatomy applications in mobile devices

Sertel $\mathrm{S}^{*}$, Meyvac1 T**

*Department of Anatomy, Faculty of Medicine Kocaeli University, Kocaeli, Turkey; **Faculty of Medicine Kocaeli University, Kocaeli, Turkey

Objectives: The aim of the study was to investigate the anatomy applications that can be downloaded to mobile devices in terms of quality and quantity.
Methods: Nowadays, applications containing products of one of the operating systems used in mobile device (smart phone, tablet etc.); Apple application store were reviewed.

Results: There are approximately 482 Applications in the apple application store. 125 free and 357 paid applications can be reached in Apple aplication store. Paid applications are priced between 1.99-169.99 tl. Paid applications are often priced at $1.99,6.49$ ve $10.49 \mathrm{tl}$. İn this system, applications contain anatomy education, terminology, pronunciation, dissection, clinical, radiologic, topographic, slice, macroscopic, microscopic, special anatomy and quiz programs. The reliability and feasibility of anatomy and recommendations in these applications were controversial $(\mathrm{p}<0,05)$.

Conclusion: It was reported that $60 \%$ of the population in developed countries is currently using smart applications in their mobile phones. In our country this ratio is $20 \%$ and is increasing rapidly. Anatomy is a major part of health professions education nowadays and it is possible to take advantage of tecnology more with regard to this issue. With the guidance of anatomist, this technology can be used in more reliable and efficent way.

Keywords: Anatomy, education, mobile applications

\section{0-18}

\section{Anatomical landmarks in endoscopic transsphenoidale} pituitary surgery

\section{Uygun $\mathrm{S}^{*}$, Berker $\mathrm{M}^{* *}$}

*Department of Anatomy, Faculty of Medicine, Hacettepe University, Ankara, Turkey; ${ }^{* *}$ Department of Neurosurgery, Faculty of Medicine, Hacettepe University, Ankara

Nowadays, Endoscopic transsphenoidal approach is accepted as the standard method for pituitary adenomas. Endoscope presents two-dimensional panoramic view of surgical field. The orientation of the surgical field becomes easier by the effective use of endoscope in short time and finding anatomic landmarks. Anatomic landmarks are the most important anatomic formations that surgeon has to find to form safe surgical field. Endoscopic Transsphenoidal Pituitary Surgery is carried out in four stages including preoperative preparation, nasal stage, sphenoidal stage and sellar stage. Superior-middle-inferior nasal conchas and sphenoid ostium are landmarks of nasal stage. By going forward through the inferior line of Concha Nasalis, concha nasalis media is leaned to lateral and by doing this concha nasalis superior becomes visible. Concha nasalis superior is approximately $1,5 \mathrm{~cm}$ above choanae and ostium sphenoidale with 1-2 $\mathrm{mm}$ diameter is just at the bottom of concha nasalis superior. Sphenovomerine suture is used for passing from nasal stage to sphenoidal stage. When sphenovomerine suture is removed, sellae base becomes clearly visible. Then, the sellae base becomes a surgical field with carotid prominence at the laterals and field from planum sphenoidale to clivus. Tumor is removed by preserving neurovascular structures. After the tumor is removed, it is checked whether there is CSF leak or not. If there is no leak, sellar reconstruction is unnecessary. If dura mater is preserved, it 
is re-placed and surgical field is covered with surgicel-spongostan. Preserved part of sinus mucosa is laid on surgical field and then the operation is finished.

Keywords: Endoscopic transsphenoidale pituitary surgery, sphenoid ostium, sphenovomerine suture, internal carotic artery, landmark

\section{0-19}

Morphological evaluation of the posterior cranial fossa dural openings in baby cadavers

Özdoğmuş Ö*, Saban E*, Özkan $M^{*}$, Yıldız SD*, Verimli U*, Çakmak Ö*, Arifoğlu Y**, Şehirli ÜS*

*Department of Anatomy, Faculty of Medicine, Marmara University, Istanbul, Turkey; **Department of Anatomy, Facult of Medicine, Bezmialem University, Istanbul, Turkey

The morphometric relationship among the cranial nerves piercing dura mater in the petroclival region is of utmost importance in posterior cranial fossa surgeries. This study aims to detect how this situation alters in baby cadavers. Reciprocal distances between the points where the crainal nerves pierce dura mater were measured in 20 baby cadavers and the results were evaluated via paired t-test using GraphPad Prism v 5.0 statistical software. According to the statistical results obtained, a significant difference was observed in between the points bilaterally on the right and the left side, where facial nerve, vestibulocochlear nerve, glossopharyngeal nerve, vagus nerve and accessory nerve pierce the dura mater (left $4.225 \pm 0.734$, right $3.925 \pm 0.634$ ). To the best of our knowledge this study is the only morphometric evaluation regarding the baby cadavers in the literature. Identifying the dural inlets and outlets of the cranial nerves is of utmost importance in sparing these structures in surgical procedures and contributes to the success rates in such surgeries.

Keywords: Posterior cranial fossa, fetus, dural outlets, morphometry

\section{0-20}

\section{Effects of chronic agomelatine treatment on dorsal hippocampal neuron numbers}

$\underline{\text { Soztutar E}}^{* * *}$, Can ÖD***, Demır Ozkay Ü***, Ucel UI***, Ulupinar $\mathrm{E}^{*}, * *$

*Department of Anatomy, Faculty of Medicine, Eskisehir Osmangazi University, Eskisehir, Turkey; **Department of Health Science Institute, Interdisciplinary Neuroscience, Eskisehir Osmangazi University, Eskisehir, Turkey; ***Department of Pharmacology, Faculty of Pharmacy, Anadolu University, Eskisehir, Turkey

Both in experimental animal studies and human brain imaging studies, it has been shown that volume of the hippocampus decreases in depression cases. Pharmacologic and somatic antidepressant treatments might increase the hippocampal neurogenesis and restore the negative effects on hippocampal atrophy by increasing the neurotrophic factors expression. Agomelatine is a novel antidepressant acting as a MT1 and MT2 melatonin recep- tor agonist and a 5-HT2C receptor antagonist. It has positive effects on learning and memory functions in addition to its anxiolytic effect. However, in previous studies, short-term effects of this drug have been investigated whereas antidepressant treatment lasts for long-terms and their effects on central nervous system appear more slowly. Therefore, in this study, we aimed to investigate the effects of chronic (20 weeks) Agomelatine administration on hippocampal neuron numbers by using stereological methods. Male Sprague-Dawley rats, reared in standard conditions, were grouped as controls and Agomelatine-treatment (40 $\mathrm{mg} / \mathrm{kg}$ oral doses) groups. After behavioral tests, rats were sacrificed via cardiac perfusion and their brains were dissected. Serial sections that are containing the dorsal hippocampus stained with Nissl method. In these sections, total neuron numbers in the dentate gyrus (DG) and cornu ammonis (CA1-3) regions of the hippocampus were estimated by using the optical fractionator probe of the Stereoinvestigator program. In Agomelatine treatment group, both granular cell number in the dentate gyrus (330.486 \pm 72.158$)$ and pyramidal neuron numbers in the CA1-3 regions $(469.596 \pm 158.247)$ were significantly higher than those of control groups $(207.611 \pm 30.195$ and $246.261 \pm 23.712$, respectively). These results suggest that, in addition to antidepressant, anxiolytic and regulatory effects of the sleep cycle, chronic Agomelatine treatment has nootropic effects and stimulates the hippocampal neurogenesis.

This study was supported by the Scientific Research Commission of the Anadolu University, Grant \#: 1209F139.

Keywords: Agomelatine, Hippocampus, Optical Fractionator, Dentate Gyrus

\section{0-21}

Anatomic variations of intrahepatic bile ducts: analysis of intrahepatic cholangiography in 46 donor hepatectomies

Dirican $\mathrm{A}^{*}$, Soyer $\mathrm{HV}^{*}$, Kose $\mathrm{E}^{* *}$, Kinacı E*, Cuglan $\mathrm{S}^{*}$, Ates $\mathrm{M}^{*},{ }^{* *}$, Ozbag $\mathrm{D}^{* *}$

*Department of General Surgery, Faculty of Medicine, Inonu University, Malatya, Turkey; **Department of Anatomy, Faculty of Medicine, Inonu University, Malatya, Turkey

Objectives: Variations in intrahepatic bile ducts (IHBD) is a challenge for hepatic resections. The aim of this study was to describe intrahepatic branching patterns of bile ducts to perform safe liver resections.

Methods: Variations manifested by intraoperative cholangiography in patient underwent donor hepatectomy for living donor liver transplantation was enrolled. Couinaud classification (1957) was used to classify the anatomical variations in IHBDs.

Results: The number of patients included in this study was 46. There were 21 male and 25 female donors. The mean age was 33.6 (19-60). The anatomy of IHBDs was typical in 26 cases (56.5\%). Triple confluence in five cases $(10.8 \%)$, anomalous drainage of the right posterior segmental duct into the common hepatic duct in 11 cases $(23.9 \%)$, presence of an accessory duct to the common hepatic duct in one case $(2.1 \%)$, unclas- 
sified or complex variations in three cases $(6.5 \%)$ were shown. There was no other defined type of variation.

Conclusion: Atypical pattern of IHBDs was not negligible (43.5\%) especially for surgeons dealing with living donor liver transplantation. To avoid unwanted biliary complications, liver surgeons should be alert to the possible variations.

Keywords: Intrahepatic bile duct, classification, anomaly

\section{0-22}

Three dimensional remodelling of temporal bone as multipurpose education and training material

Taser $\mathrm{F}^{*}$, Akcer $\mathrm{S}^{*}$, Oghan $\mathrm{F}^{* *}$, Sanal B $\mathrm{B}^{* * *}$

*Department of Anatomy, Faculty of Medicine, Dumlupinar University, Kütahya; ${ }^{*}$ Department of ENT, Faculty of Medicine, Dumlupinar University, Kütahya; ***Department of Radiology, Faculty of Medicine, Dumlupinar University, Kütahya

Temporal bone is very complicated bone with outer, middle and inner ear structures on its outer and inner surfaces. Since temporal bone is a small bone, demonstration of all these structures is very difficult on anatomy education and practices. Also temporal bone dissection is very important during residency training of ENT as surgical method. It is very difficult to find out a cadaver for this dissection training courses. So we aimed that remodeling of temporal bone using appropriate computer programs and three dimensional printing it several times enlarged dimensions using similar materials with bone to use more effective at student education and resident training and dissection programs on this study. CT DICOM images of a patient without any deformity on temporal bone with $0.5 \mathrm{~mm}$ slice thickness were converted to three dimensional images and transformed an appropriate format to print out on $3 \mathrm{D}$ printer. These 3 to 5 times enlarged models were printed using first plastic materials and then calcium phosphate. Middle and inner ear structures were exposed with temporal bone dissection. The anatomic models made with this method could be use for student and resident education, also practices of surgical methods on temporal bone.

\section{0-23}

Effects of grape seed extract and vitamin E on oxidative stress and apoptosis in the hippocampus of streptozotocin-induced diabetic rats

Yonguc GN*, Dodurga $Y^{* *}$, Adiguzel E***, Gundogdu G***, Kucukatay $\mathrm{V}^{* * * *}$, Ozbal S***** , Yilmaz I $\mathrm{I}^{\star * * * * *}$, Cankurt $\mathrm{U}^{* * * * *}$, Yilmaz $\mathrm{Y}^{* * * * * * *}$, Akdogan I***

*Department of Anatomy, Faculty of Medicine, Izmir University, Izmir, Turkey; **Department of Medical Biology, Faculty of Medicine, Pamukkale University, Denizli, Turkey; $* * *$ Department of Anatomy, Faculty of Medicine, Pamukkale University, Denizli, Turkey, $* * * *$ Department of Physiology, Faculty of Medicine, Pamukkale University, Denizli, Turkey; *****Department of Histology and Embryology, Faculty of Medicine, Dokuz Eylul University, Izmir, Turkey; $\star \star \star \star * \star$ Department of Pharmacology, Izmir Bozyaka Training and Research Hospital, Izmir, Turkey; $* * * * * *$ Department of Food Engineering, Faculty of Engineering and Architecture, Mehmet Akif Ersoy University, Burdur, Turkey
The aim of the present study is to show the effects of Grape Seed Extract (GSE) and vitamin E (Vit E) on oxidative stress and apoptosis in the hippocampus of streptozotocin induced diabetic rats. Control, diabetic, diabetic treated with GSE (Diabetic+GSE) and vitamin E (Diabetic+Vit E) (n=6) groups were used. GSE and vitamin $\mathrm{E}$ given orally $(100 \mathrm{mg} / \mathrm{kg} /$ day $)$ during six weeks. Following parameters were evaluated; oxidative stress index (OSI), TUNEL staining and Bcl-2, Bcl-XL, Bax, Caspase-3, -9, -8, Cyt-c, TNF- $\alpha$, NF-kB gene expressions. OSI levels were significantly increased in plasma and hippocampus of Diabetic group compared to control, and decreased in Diabetic+GSE and Diabetic+Vit E groups compared to diabetic. TUNEL positive neurons significantly increased in hippocampus of diabetic group compared to control, and decreased in the Diabetic+GSE and Diabetic+Vit E groups compared to diabetic. This decrease was more prominent in Diabetic+GSE group. In the hippocampus of Diabetic group, Bcl-2, Bcl-XL gene expressions were significantly decreased; Bax, Caspase-3, 9, -8, Cyt-c, TNF- $\alpha$, NF-kB gene expressions were significantly increased compared to control. In Diabetic+GSE and Diabetic+Vit E groups Bcl-2 gene expressions were significantly increased, Bcl-XL gene expression did not differ compared to diabetic group. In Diabetic+GSE group expression of Bax, Caspase-3, -9, -8, Cyt-c, TNF- $\alpha$, NF-kB genes; in Diabetic+Vit E group expression of Caspase-3, -9, TNF- $\alpha$, NF-kB genes were significantly decreased compared to diabetic. In conclusion, these findings suggest that GSE (more prominently) and vitamin $\mathrm{E}$ decrease oxidative stress and neuronal apoptosis occurring in the hippocampus of streptozotocin diabetic rats. This study was supported by Pamukkale University Scientific Research Fund (2011BSP026). Preparation of the grape seed extract which was used for treatment was supported by The Scientific and Technological Research Council of Turkey (TUBITAK, SBAG-3994.108S157) and Pamukkale University Scientific Research Fund (2008TPF005). This abstract has been accepted for a poster presentation in 27th European College of Neuropsychopharmacology Congress in Berlin, on 18-21 October 2014 and has been selected for a Grant.

Keywords: Diabetes, grape seed extract, vitamin E, hippocampus, oxidative stress, apoptosis

\section{0-24}

Tomographic evaluation of bone morphology in posterior mandibular region for safe placement of dental implants

Yildiz S*, Bayar GR**, Guvenc I***, Kocabiyik N*, Cömert $\mathrm{A}^{* * * *}$, Yazar $\mathrm{F}^{*}$

*Department of Anatomy, Gulhane Military Medical Academy, Ankara, Turkey; **Department of Oral and Maxillofacial Surgery, Gulhane Military Medical Academy, Ankara, Turkey; ***Department of Radiology, Gulhane Military Medical Academy, Ankara, Turkey; $* \star * *$ Department of Anatomy, Ankara University, Faculty of Medicine, Ankara, Turkey

The aim of this study was to examine the morphology of submandibular fossae at edentulous posterior regions of dried mandibles, and to determine a safe range for proper lingual 
angulation during the placement of a dental implant in the posterior mandibular region, with a computerized tomographic scan study. Spiral computed tomographic images of 78 dry adult human mandibles were evaluated to determine the deepest area in the submandibular fossa. Then, the proper lingual angulations for the placement of a dental implant at these regions were measured. Pearson's correlation coefficient was calculated to show the relation between the depths of submandibular fossa and lingual implant angulations. Differences between the lingual implant angulations, and the depths of submandibular fossa on each side of the mandibles, "Paired T-Test" was used. Depths of the submandibular fossa and lingual implant angulations varied between 1.1-4.6 mm, 62-84 degrees on right side of the mandibles, and 1.1-4.5 mm, 65-83 degrees on left side of the mandibles. There was statistically moderate negative correlation between the degree of lingual implant angulations and the depth of submandibular fossa on each side of the mandible. Within the limits of this study, the depth of the submandibular fossa was measured as $\geq 2 \mathrm{~mm}$ in around $71.5 \%$ of examined regions, and lingual implant angulations were between 62-84 degrees. These results may be considered by clinicians who are planning the dental implant placement in posterior mandible to avoid potential risk of lingual cortical plate perforation.

Keywords: Dental implant, submandibular fossa, perforation

\section{0-25}

Morphometric and morphologic description of foramen occipitale and foramen mastoideum for surgical applications in suboccipital region

Türk F* Akyer ȘP* $^{*}$ Özdemir $M^{* *}$, Akdoğan I*

*Department of Anatomy, Faculty of Medicine, Pamukkale University, Denizli, Turkey; ${ }^{*}$ Department of Neurosurgery, Faculty of Medicine, Pamukkale University, Denizli, Turkey

Objectives: The description of emissary veins connecting plexus venosus suboccipitalis with cranial venous sinuses is important for surgery. The detailed morphometric and morphologic descriptions of foramen occipitale (FO) and foramen mastoideum (FM) inform about the presence and the locations of emissary veins through these foramina, and is scarce in the literature.The objective of this study was to investigate the presence, locations, numbers and sizes of FO and FM.

Methods: In the present study, 53 dried adult human skulls were examined in the Anatomy Department of Pamukkale University. The patency of the foramen in each case was confirmed by passing a probe.The distances between FM and processus mastoideus ( $\mathrm{PM})$, and asterion, the distances between FO and crista occipitalis externa (COE), and protuberentia occipitalis externa (POE), and posterior border of foramen magnum (FMa), and the diameters of FM and FO were measured by caliper.

Results: The overall prevalence of FO was \%32.6 on the right and \%30.4 on the left, the overall prevalence of FM was \% 79.5 on the right and \%71.4 on the left. The mean distance between $\mathrm{FO}$ and posterior border of FMa was a $18.54 \mathrm{~mm}, \mathrm{FO}$ and COE was a $6.43 \mathrm{~mm}, \mathrm{FO}$ and POE was $28.30 \mathrm{~mm}$. The mean distance between FM and asterion was $20.73 \mathrm{~mm}, \mathrm{FM}$ and PM was $31.09 \mathrm{~mm}$. The mean diameter of the $\mathrm{FO}$ was $1.12 \mathrm{~mm}$ on the right and $1.00 \mathrm{~mm}$ on the left. The mean diameter of the FM was $1.47 \mathrm{~mm}$ on the right and $1.71 \mathrm{~mm}$ on the left.

Conclusion: The detailed description of variational differences of the locations, presence, numbers of FO and FM and their properties about placement and size are important to create awareness and guidance for surgeons making surgery in this region.

Keywords: Emissary vein, occipital foramen, mastoid foramen, suboccipital surgery

\section{0-26}

\section{The effects of preobesity to quality of life in patients with ankylosing spondylitis}

\section{Toy $\mathrm{S}^{*}$, Ozbag D*, Altay Z**}

*Department of Anatomy, Faculty of Medicine, Inönü University, Malatya, Turkey; **Department of Physical Therapy and Rehabilitation, Faculty of Medicine, Inönü University, Malatya, Turkey

Objectives: Ankylosing spondylitis (AS) is a prototype of disease of spondyloarthropathies which is chronic and charactarized by inflammation. As in many chronic diseases, quality of life is affected negatively by the disease in patients with ankylosing spondylitis. Body Mass Index (BMI), total body fat which is correlated with the weight is found by weight divided by square of height (weight $(\mathrm{kg}) /$ height $(\mathrm{mC})$ )). This method is currently the most commonly used methods. In this study we aimed to investigate the factors that affect quality of life in patients with AS.

Methods: 28 patients, diagnosed AS according to the Modified New York Criteria, who applied to Inonu University Medical Faculty Physical Theraphy and Rehabilitation clinic were included in the study and control group was selected as 30 healthy adults. The patients were matched with the control group by age, gender, body mass index (BMI), education and work status. The disease activity was measured by Bath AS disease activity index (BASDAI) and functional status was measured by Bath AS functional index (BASFI). ASQoL was used to evaluate the quality of life of patients with AS, Short Form - 36 (SF-36) was carried out to the patients by comparing with the control group.

Results: High Body Mass Index (BMI) and low education status have been determined as the factors that affect the quality of life in patients with AS. Besides, we observed that BASDAI values were higher in women patients. Compared to the control group, the quality of life were worse in patients with AS. We have observed there was a meaningful correlation between increases BASFI scores and high BMI in patient with AS.

Conclusion: High BMI in patients with AS is one of the factors which affecting the quality of life. In which area of life is found to be affected we believe that it would be in terms of guiding strategies.

Keywords: Ankylosing spondylitis, body mass index (BMI), preobesity, quality of life 


\section{0-27}

Properties of glutamic acid decarboxylase distribution in the septal area GABAerjic neurons

Verimli U, Sehirli US

Department of Anatomy, Faculty of Medicine, Marmara University, Istanbul, Turkey

The septal area, a limbic prosencephalon region involved in the pathogenesis of certain disorders such as epilepsy, is briefly composed of Lateral (LS) and Medial Septal Nuclei(MS). There are controversies regarding the distribution of gamma amino-butyric acidergic (GABAergic) neurons of these two nuclei in the literature. The main inconsistency is about the intensity difference of the GABA synthesizing enzyme Glutamic Acid Decarboxylase (GAD). General opinion is that the GABAergic neurons in the region posses GAD-67 subtype more in density. On the contrary, the GAD-65 subtype is known to be located in the synaptic terminals and is the subtype responsible for the synaptic functions. The aim of our study is to test this inconsistency regarding the enzyme subtype distribution in this region. In this study we used 5 GAD-65 and 5 GAD-67 23 days old transgenic mice brains. We acquired consecutive sections and stained them via anti green fluorescence protein (anti- GFP). One out of five sections were examined and neuron count was performed. In order to eliminate the risk of false positive staining, cortical regions were also examined and similar staining was detected in both subtypes. The results obtained from both subtypes were compared statistically via student t-test. According to our findings, GAD-65 subtype is more prominent in both nuclei in contrast to the literature $(\mathrm{p}<0.0001)$. We think the results of the current study may contribute to solving the controversies in the literature regarding the enzyme subtype intensity of the region.

\section{$0-28$}

\section{Investigation of corpus callosum by planimetry methods in patients with temporal epilepsy}

Caglar $\mathrm{V}^{*}$, Alp SI ${ }^{* *}$, Tugtag B*, Sener $\mathrm{U}^{* * *}$, Ozen OA*, Alp $\mathrm{R}^{* * * *}$

*Department of Anatomy, Faculty of Medicine, Namik Kemal University, Tekirdag, Turkey; **Vocational School of Health Service, Namik Kemal University, Tekirdag, Turkey; $* * *$ Department of Physiology, Faculty of Medicine, Namik Kemal University, Tekirdag, Turkey; ${ }^{* * * *}$ Department of Neurology, Faculty of Medicine, Namik Kemal University, Tekirdag, Turkey

Objectives: The purpose of this study was to evaluate the effects of temporal epilepsy on corpus callosum morphometry in patients with temporal lobe epilepsy (TLE).

Methods: The diagnosis of the epileptic syndrome was based on International League Against Eplepsy criteria and this study was conducted on MR images of 25 epilepsy patients and 26 control subjects. We classified the patients according to their duration of epilepsy: $<10$ and ? 10 years. Sex and age were studied for demographics. Furthermore, duration of epilepsy were studied for the clinical evaluation. Digitally traced boundaries of the surface areas were automatically calculated for midline section and the machine administered the projection area of the corpus callousm.
The projection area length of the corpus callosum was also estimated.

Results: We found statistically significant atrophy in CC of individuals who are 25 years or older. The corpus callosum of patients with TLE was determined to be smaller than healthy indivuduals. In gender comparisons of patients with TLE is found that CC of women is smaller than men's. There is a relationship between epilepsy duration and atrophy in CC.

Conclusions: The present study shows a clear influence of TLE on the structure of the CC. The duration of epilepsy is the most important factors to determine of influence of TLE on CC.

Keywords: Corpus callosum, temporal lobe epilepsy, PAL value, $M R$

\section{0-29}

\section{Assessment of radiological angular measurements on the patients with the foot pain complaints}

\section{Deniz $G^{*}$, Kaya A**, Kavakli A*, Ogeturk $M^{*}$}

*Department of Anatomy, Faculty of Medicine, Firat University, Elazig, Turkey; **Department of Physical Medicine and Rehabilitation, Faculty of Medicine, Firat University, Elazig, Turkey

Objectives: Foot pain is one of the common pathology in the middle ages. Among the causes of foot pains are epin calcanei, rheumatic diseases and medial longitudinal arch problems like pes planus, and pes cavus. There are lots of methods to assess these diseases. In our study, we aim to compare foot radiography of the patients with foot pain complaints and healthy individuals to search the relationships between foot pathologies.

Methods: This study was included 30 pes cavus, 30 pes planus, 30 epin calcanei and 30 control groups (between the ages of 30 60). All individuals' right and left foot lenght, metatarsophalangeal width, calcaneus pitch, talo-horizontal, talo-first metatarsal and lateral talocalcaneal angles of lateral radiographies were evaluated. On the statistical analysis, multivariete varians and correlation analysis were used.

Results: There was no statistically significant difference in all the cases of gender, age, weight and body mass index $(p>0,05)$. For the calcaneus pitch angles; $95 \%$ pes cavus, $32 \%$ pes planus, for the talo-first metatarsal angles; $88 \%$ pes cavus, $96 \%$ pes planus, and for the lateral talocalcaneal angles; $57 \%$ pes planus were diagnosed. Between the calcaneus pitch angles of epin calcanei and pes planus and lateral talocalcaneal angles a very strong positive correlation ( $\mathrm{r}=0.800, \mathrm{r}=0.619, \mathrm{p}<0.001)$, and between the calcaneus pitch angles and talo-horizontal angles a significant positive correlation $(\mathrm{r}=0.457, \mathrm{r}=0.502, \mathrm{p}<0,05)$ was found. On the patients with epin calcanei, $10 \%$ pes cavus, and $90 \%$ pes planus were found.

Conclusion: The angular change that occurs resulting from deformation of food biomechanics causes various deformities. The beginning of these deformities, pes planus comes first. Therefore, we believe that in researching the patients with foot complaints, considering the interrelated deformities would be helpful to plan the treatment.

Keywords: Foot pain, radiography of foot, anthropometry. 


\section{0-30}

Importance of corona mortis in totally extraperitoneal inguinal herni repair: single center experience

$\underline{\text { Ates }}^{*},{ }^{* *}$, Kose $\mathrm{E}^{* *}$, Kınac1 $\mathrm{E}^{*}$, Sarıcı B*, Cuglan $\mathrm{S}^{* *}$, Korkmaz MF**, Dirican $\mathrm{A}^{*}$

*Department of General Surgery, Faculty of Medicine, Inonu University, Malatya, Turkey; **Department of Anatomy, Faculty of Medicine, Inonu University, Malatya, Turkey

Objectives: With the advances in laparoscopic surgery, laparoscopic hernia repair getting popularity in last two decades. Totally extraperitoneal (TEP) inguinal hernia repair is one of the methods. This method can be associated with vascular complications especially during the fixation of the mesh. A rare but important nominee is so called 'Corona Mortis or Crown of Death" an anastomosis between obturator vessels and external iliac vessels. It can be damaged accidentally during TEP procedure. In this study, the preperitoneal inguinal vascular anatomy of patients underwent TEP procedure were evaluated.

Methods: 317 patients underwent TEP procedure from January 2005 to July 2014 were retrospectively evaluated. Prepertioneal vascular anatomy of the patients were evaluated.

Results: The hernia was unilateral in 243 (76.6\%) patients and bilateral in 74 (23.4\%) patients. Of these 391 hernia, 243 were direct inguinal hernia, 129 were indirect, 19 were femoral hernia. In patients underwent bilateral laparoscopic inguinal dissection (LID), there was a thick arterial connection (an aberrant obturator artery - AOA) between the inferior epigastric artery and obturator artery in four cases bilaterally and in two cases unilaterally (right sided). Nine patients had bilateral irregular arterial plexus which is generated by connection between an irregular AOA running over the superior pubic ramus and an aberrant inferior epigastric artery (AIEA) running medially over the inguinal ligament towards the pubis. In patients underwent unilateral LID, 12 of them had thick AOA connection and an arterial plexus as decribed above was evident in 69 patients. Additionally in 65 cases (14 bilateral and 51 unilateral) there was a pubic branch of epigastric artery (AIEA) running next to inguinal ligament towards the pubis but not form an anastomosis. We did not encounter any venous anastomoses in this study. It could be due to the collapse in veins under the pressure of $14 \mathrm{mmHg} \mathrm{CO} 2$ that was used to obtain preperitoneal space. Finally, of these 317 patients, in 96 (30.2\%) patients there was an arterial plexus can be called as Corona Mortis at least in one side and in 161 (50.8\%) patients there was a vulnerable arterial structure at least in one side.

Conclusion: Laparoscopic hernia repair or any other surgical producers performed in the preperitoneal space require an extra attention to prevent unwanted injuries to vascular structures. During TEP procedure, tacks applied to the hernia sac or to the mesh on cooper ligament can damage the Corona Mortis or pubic branch of inferior epigastric artery (AIEA) and can cause an uncontrollable bleeding. The risk of presence of vulnerable vessels can reach to nearly $50 \%$.

Keywords: Laparoscopic surgery, extraperitoneal inguinal herni, corona mortis

\section{0-31}

Arachnoid granulation and dural venous sinuses of the subdural space in terms of anatomical and radiological evaluation

$\underline{\text { Imre }} \mathrm{N}^{*}$, Kocabiyik N*, Guvenc I**, Yazar F*

*Department of Anatomy, Gulhane Military Medical Academy, Ankara, Turkey; **Department of Radiology, Gulhane Military Medical Academy, Ankara, Turkey

Arachnoid granulations were first described 300 years ago by Antonia Paccione in spite of the still neuroimaging literature, there is a lack of knowledge on this subject. For example, the importance of the relationship of the vessels of the arachnoid granulations is uncertain. Ever arachnoid granulations were reported with in the cerebral venous sinus lumen along with Cerebral venography, contrast CT and conventional MR. Arachnoid granulations numbers increase with age and are known to be more prominent. We aimed to evaluate this granulations in terms of age, gender, size, frequency and localisation with $3 \mathrm{~T}$ devices. Contrast Enhanced MR images of the brain were examined retrospectively. Axial T2-weighted, T2-weighted coronal and axial T1-weighted contrast applied, coronal, and sagittal measurements were made from images. According to the dural sinus, localisations of the aracnoid granulations were evaluated and their size were measured. This study carried out 3T MR imagines obtained from 38 people. Arachnoid granulations were found in the superior sagittal sinus of the frontal section 16 , of the parietal section 43 , of the occipital section 8. Arachnoid granulations were found 9 in right tranvers sinus, 12 in left transvers sinus, 10 in straight sinus. Arachnoid granulations sizes were range from 1 to 6.5 (average 2.30) $\mathrm{mm}$. For some inexperienced physicians, these granulations with dural venous sinus thrombosis or tumors within the sinus can misleading. In this study, mapping anatomical of the arachnoid granulation is aimed. Obtained data from this study should be considered in radiological and clinical evaluations.

Keywords: Arachnoid granulation, subarachnoid cavity, 3T MR

\section{0-32}

Formation of solid tumor by using low number tumor cells in Ehrlich ascites tumor model

Ertekin T*, Nisari $\mathrm{M}^{*}$, Sarıca $\mathrm{ZS}^{* *}, \mathrm{Al} \mathrm{Ö}^{*}$, Ülger $\mathrm{H}^{*}$

*Department of Anatomy, Faculty of Medicine, Erciyes University, Kayseri, Turkey; ${ }^{* *}$ Experimental and Clinical Research Center, Erciyes University, Kayseri, Turkey

Cancer is the most progressive and devastating disease that threat of mortality to the entire world despite significant advances in medical technology for its diagnosis and treatment. The Ehrlich mouse Ascites Tumor has become one of the widely used experimental cancer model. In this study, we have investigated relationship between the formation rate of solid cancer and injection of a low number of Ehrlich ascites tumor (EAT) cells. 24 male Balb/C mice were divided into 3 groups: Experimental groups 1 (n: 8) and 2 (n: 8) were injected subcutaneously with $1.5 \times 10^{5}$ and $3 \times 10^{5}$ EAT cells to induce solid tumor growth respectively. The control 
group (n: 8) were treated with normal saline ( $0.1 \mathrm{ml} / \mathrm{mouse})$. The changes of body weight and tumor volume in all animals were recorded. Although body weights of two experimental groups were elevated because of the tumor mass but statistical differences was not determined among the groups. Initially tumor formation was induced in all of the animals of experimental groups, but subsequently the tumor mass was lost in 2 and 3 mice of experimental groups 1 and 2, respectively. Although inbred animals were used in this study, we suggest that individual differences of mice may cause this situation. However, further studies with more animals should be made and the probable causes of tumor reduction and disappearance should be investigated. Also, this case should be taken into account in studies that cancer models are formed.

Keywords: Ehrlich tumor, experimental model, mice

\section{0-33}

\section{The localisation of carotid bifurcation according to various anatomical landmarks}

Kürkçüoğlu A*, Pelin $C^{*}$, Öktem H*, Aytekin $C^{* *}$

*Department of Anatomy, Faculty of Medicine, Baskent University, Ankara, Turkey; **Department of Radiology, Faculty of Medicine, Baskent University, Ankara, Turkey

Objectives: For the surgical procedures performed in head and neck region the anatomical vascular variations should be taken into consideration. Surprisingly, there is a paucity of published reports on the anatomy of the carotid bifurcation (CB). Furthermore, there is a lack of consensus among various anatomy texts as to the correlation of the common carotid artery (CCA) and its surrounding structures. To help minimize operative morbidity and mortality in patients undergoing operations, involving the CCA, a clear understanding of the anatomy of the region is essential. The aim of this study was to describe the carotid bifurcation (CB) to its surrounding structures to locate it properly by using different landmarks.

Methods: Angiographical images of 68 male and 32 female a total of 100 subjects were evaluated, and the CB levels were investigated according to the cervical vertebrae, hyoid bone and mandibular angle level. The study was approved by the ethical committee of in Baskent University Hospital (KA 14/19).

Results: When the levels of CB were evaluated; two bifurcations were observed at the level of $\mathrm{C} 2$ vertebral body, one bifurcation at $\mathrm{C} 7$ vertebral body, and most of the CCA bifurcations were observed at the level of the intervertebral disc between $\mathrm{C} 4$ and $\mathrm{C} 5$ vertebra bodies (\%31.6) in males. In females, the most commonly observed bifurcation level was at C4 vertebral body (right side; \%26.3 and left side\% 15.7). The distances of CAA to mandibular angle and hyoid bone were also evaluated. No significant side difference and sex difference with the above mentioned parameters were observed.

Conclusion: Detailed information on the branching pattern of the common carotid artery and its bifurcation levels will prevent the clinician causing a vascular injury during catheterization in radiological interventions or surgical approaches.

Keywords: Carotid bifurcation, common carotid artery, variation

\section{0-34}

The comparison of the anatomical terminology in the last 25 years

Ocak $M^{*}$, Akdemir Aktaş $\mathrm{H}^{*}$, Uzuner $\mathrm{MB}^{*}$, Geneci $\mathrm{F}^{*}$, Aşkit Ç**, Sargon MF*

*Department of Anatomy, Faculty of Medicine, Hacettepe University, Ankara, Turkey; ** Faculty of Language, History and Geography of the United States in Latin Language and Literature, Ankara University, Ankara, Turkey

The Anatomical terminology forms the basis of all health sciences and it is very important in communication skills in all over the world. Since the 19th century, the terminology is renovating continuously in accordance with the scientific innovations and clinical requirements. In this study, the changes in Anatomical terminology observed in the last 25 years were detected. The changes in Anatomical terminology were examined in Nomina Anatomica (1989), FCAT's List (1996) and Terminologia Anatomica (1998). In their comparison, 129 terms were removed from the terminology and 1359 terms were added from Nomina Anatomica to Terminologia Anatomica. 196 of these terms were added after the FCAT's List. Additionally; in the last 25 years, grammar changes were done in 563 terms. In the examination of the newly added terms to Anatomy terminology, we can conclude that these terms were still be used in the ancient anatomy text-books, but they were not present in the ancient terminology books. In the last two terminology text-books, they were all added. In the examination of the FCAT's list it was detected that some terms were removed from the terminology, however they were once more added to terminology in Terminologia Anatomica. The other reasons of newly added terms to terminology can be listed as making the regional classifications more clear, scientific innovations and lack of usage of some terms in clinical applications. The reasons of grammar changes in Latin words can be listed as follows: To define the accurate localizations of the anatomical structures more clearly, singular and plural term changes for better descriptions, to make the names of arteries and nerves' branches more understandable and to give the details of anatomical structures in their names. At last, the whole content of three Nominas was compared according to general organization. In Nomina Anatomica, there was a special section for histology and embryology; however in Terminologia Anatomica; these histologic terms were not found separately, instead, were taking part under gross anatomy. Secondly; the English terminology just near to their Latin terms were added to Anatomical terminology. Then; it was observed that the indexes of Latin terminology and English terminology were included to Terminologia Anatomica and the eponyms were at first added to FCAT' List. Sex symbols were also added to the terminology in FCAT at first. As a result of all these innovations, we can conclude that the Anatomical terminology is getting larger day by day and these newly added terms are bringing a new scientific approach to the science of anatomy.

Keywords: terminologia anatomica, nomina anatomica, nomenclatura anatomica 


\title{
Poster Presentations
}

\author{
(P-1 - P-165)
}

\section{P-1}

Manufacturing solid replicas of the basal nuclei (the basal ganglia) of the human brain by using fused deposition modeling (FDM) technique

Kapakin S

Department of Anatomy, Faculty of Medicine, Ataturk University, Erzurum, Turkey

The aim of this study was to fabricate tangible hard copies of the basal nuclei of the human brain by using FDM Technique. We used imaging data from the visible human dataset, which belongs to the visible human project (VHP), introduced by the National Library of Medicine (NLM). The visible human data consists of different modalities: CT, MRI, and anatomical cryosections. In this study, anatomical cryosections were used since they have high contrast and resolution than the other modalities. The cryosections of interest were used to reconstruct the basal nuclei of the human brain three-dimensionally. These CAD (Computer Aided Design) models in DXF format could be converted into STL format. These models in STL format were sent to rapid prototyping machine. FDM printing is one of the most widely used forms of $3 \mathrm{D}$ printing in which the material is deposited in layers to create a $3 \mathrm{D}$ printed object. Tangible hard copies of the caudate nucleus, putamen, globus pallidus and substantia nigra were obtained. The FDM was appropriate for office environments, and the characteristics of FDM were more favorable for operating theater conditions.

Keywords: Human brain, basal nuclei, basal ganglia, rapid prototyping, fdm, fused deposition modeling

\section{P-2}

Virtual simulation of the functional endoscopic sinus surgery

Kapakin S

Department of Anatomy, Faculty of Medicine, Ataturk University, Erzurum, Turkey

Functional endoscopic sinus surgery is the primary approach used today in the surgical treatment of sinusitis and nasal polyps. The aim of this study was to perform these operations in the virtual environment. The Visible Human Dataset (VHD) was used as the input imaging data. The Surfdriver software package was applied on these images to reconstruct the paranasal sinuses as three-dimensional (3D) DXF (Data Exchange File) models. The CAD (Computer Aided Design) model in DXF file format was transferred into Cinema 4D to be used in rendering the photorealistic image by the Advanced Render Module. These models were also post-processed in
Cinema 4D for photorealistic images. We have used surface approach to prepare a reconstructions on the paranasal sinuses. Because it is especially difficult to approach by dissection and to delineate in static images in an atlas. In this technique, it is essential to preserve normal anatomy and physiology as much as possible and protect patients from bleeding and creating a leak of cerebrospinal fluid.

Keywords: Paranasal sinuses, virtual simulation, functional endoscopic sinus surgery

\section{P-3 \\ The obese swimmers even though exercise, identification of body fat paramaters with the method of bioelectrical impedance analysis}

$\underline{\text { Sertel } S^{*}}$, Colak T*, Bamac B*, Meyvaci $\mathrm{T}^{* *}$, Unal $\mathrm{S}^{* * *}$, Tasdemir R*, Aksu E*

*Department of Anatomy, Faculty of Medicine, Kocaeli University, Kocaeli, Turkey; ${ }^{*}$ Department of Anatomy, Faculty of Medicine, Kocaeli University, Kocaeli, Turkey; ${ }^{* * *}$ Department of Nutrition and Dietetics, School of Health Sciences, Arel University, Istanbul, Turkey

Objectives: Obesity is a health problem that increases day by day even though taking precautions in our country as it happens all in the world. It main reason is the amount of energy that taken with food is more than spent energy. In some situations intensive obesity seems especially in children. In our reserarch, the aim is identification of body composition parameters with the method bioelectrical impedance analysis (BIA) that seems in obesity swimmers who has more physical activity and examine the causes.

Methods: In our research, we use BIA that is not have risk for illness and it is easy to apply to devices. Attached to swim club, determined obesity age is $10.57 \pm 1.93$ used Tanita Body Composition Analyzer TBF 410GS device for weight $(\mathrm{kg})$ and amount of body fat $(\mathrm{kg})$, fat free mass $(\mathrm{kg})$ and body fat ratio(\%) antropometric features. Assessment of all datas that used as statistical revelence level $\mathrm{p}<0.005$ and PASW (version 18 SPSS).

Results: Assessment of cases of height $(\mathrm{cm})$, weight $(\mathrm{kg})$, body mass index $\left(\mathrm{kg} / \mathrm{m}^{2}\right)$, body fat $(\mathrm{kg})$, fat free mass $(\mathrm{kg})$ and body fat ratio (\%) belonging to variation that accounts for male is respectively $152.6 \pm 9.12,58.34 \pm 16.61,24.16 \pm 5.31,16.37 \pm 10.36,32.07 \pm$ $8.79,40.15 \pm 6.39$; for female is respectively $152.90 \pm 8.94,58.93 \pm$ $16.26,24.38 \pm 5.22,18.52 \pm 10.14,36.31 \pm 8.61,38.56 \pm 6.27$.

Conclusion: It is thought that swimmers that do regular training at the result of seeing clildhood obesity arise from children wrong feeding behavior, family's inability of knowledge level about feeding and regular training of land exercises. With the BIA methods identification of weight, body mass index, body 
fat, fat free mass and body fat ratio is easy, safe and it is important for arrangement the treatment of obesity for children athletes and arrangement of feeding behavior.

Keywords: Obesity, BIA, body mass index, body fat, fat free mass and body fat ratio

\section{P-4}

Evaluation of the collodiaphyseal angle according to the body mass index in the cases osteopenia and osteoporosis

Sertel S*, Colak T*, Bamac B*, Meyvaci T**, Tasdemir R*, Dalg1c $M^{*}$

*Department of Anatomy, Faculty of Medicine, Kocaeli University, Kocaeli, Turkey; **Faculty of Medicine, Kocaeli University, Kocaeli, Turkey

Objectives: The osteoporosis is a systemic skeletal disease which is characterized with the increase in the risk of fracture as a result of the deformation in the micro-architectural structure of the bone tissue and low bone mass. The aim of this study is to evaluate whether the collodiaphyseal angle is different in the cases with osteopenia and osteoporosis having different body mass index.

Methods: In this study, the cases have been grouped according to their body mass index (BMI) and t-score in the bone density measurement report. In each group, the reports belonging to totally 180 men and 180 women have been analyzed in 30-person subgroups. The collodiaphyseal angle in the report has been measured with the digital goniometer. As the statistical significance level $\mathrm{p}<0.05$ has been determined and PASW (version, 18 SPSS) has been used.

Results: When the results belonging to the women are examined, a significant difference has been found in terms of the averages of the collodiaphyseal angle $(\mathrm{p}<0.001)$. When the differences among the groups are examined in terms of the collodiaphyseal angle in details, it has been determined that the average of the cases with osteoporosis having BKI between 18.5-24.9 $\mathrm{kg} / \mathrm{m}^{2}$ is significantly higher than the average of the cases with osteopenia having BMI between 18.5-24.9 kg/m2 and 30-39.9 $\mathrm{kg} / \mathrm{m}^{2}$ (respectively p values $0.001 ; 0.002$ ). When the results belonging to the men are examined, a significant difference has been found between the groups in terms of the averages of the collodiaphyseal angle ( $\mathrm{p}=0.030)$. When the differences between the groups are examined in details in terms of the collodiaphyseal angle variable, it has been observed that the average of the cases with osteopenia having BKI between $18.5-24.9 \mathrm{~kg} / \mathrm{m}^{2}$ is significantly lower than the average of the cases with osteoporosis having BMI between $25-29.9 \mathrm{~kg} / \mathrm{m}^{2}(\mathrm{p}=0.033)$.

Conclusion: According to these results, it has been observed that the body mass index and bone mineral density have effects on the averages of the collodiaphyseal angle in both groups. We propose the realization of the more comprehensive studies including wider case groups in this content.

Keywords: Collodiaphyseal angle, bone mineral density, body mass index
P-5

Historical development of endoscopic transsphenoidal pituitary surgery

Uygun $\mathrm{S}^{*}$, Cetin $\mathrm{N}^{*}$, Berker $\mathrm{M}^{* *}$

*Department of Anatomy, Faculty of Medicine, Hacettepe University, Ankara, Turkey; ${ }^{* *}$ Department of Neurosurgery, Faculty of Medicine, Hacettepe University, Ankara, Turkey

The first transsphenoidal surgical approach to pituitary adenomas has been performed by Herman Schloffer with superiolateral nasoethmoidal approach in Austria Innsburg on March 16,1907. Hirsch has applied endonasal transseptal transsphenoidal approach in 1910. In 1960s, by the usage of microscope in operations, endoscopic endonasal transsphenoidal approach has been being used for pituitary adenomas. The first complete endoscopic approach to sella turcica has been performed in France by Jankowski and his friends to three patients in year 1992. In 1997 at Pittsburg University, neurosurgery expert Jho and otolaryngologist Carrau have performed complete pituitary adenoma excision surgery with their 50 case series. Till now, Enrico de Divitiis and Paolo Cappabianca (Napoli), Giorgio Frank (Bologna) from Italy, Amin Kassam from USA and Mustafa Berker from Turkey have contributed to the usage of complete endoscopic techniques for both glandula pituitary surgery and pathologies related with skull base. In the following years, recognition of endoscope by brain surgeons, approach to sellar and parasellar region gained a new perspective. Nowadays, endoscopic transsphenoidal approach has taken place of microscopic surgery for brain surgeons and has become standard approach at many clinical centers.

Keywords: Endoscopic transsphenoidal pituitary surgery, endoscope, hypophysis tumour

\section{P-6 \\ Creating VRML (Virtual Reality Modeling Language) form of the visceral organs of the rabbits}

Terim Kapakin $\mathrm{KA}^{*}$, Kapakin $\mathrm{S}^{* *}$

*Department of Pathology, Faculty of Veterinary Medicine, Ataturk University, Erzurum, Turkey; **Department of Anatomy, Faculty of Medicine, Ataturk University, Erzurum, Turkey

The aim of the this study is to create VRML form of the visceral organs of the New Zealand Rabbits and to share them on the web. A total of ten adult healthy New Zealand Rabbits of both sexes aged 1.5-2 years and weighing between 3.5 and $4 \mathrm{~kg}$ were used. Animals were intravenously anaesthetized with $5 \mathrm{mg} / \mathrm{kg}$ of ketamine-HCl (KetamidorTM Richer Pharma AG, Wels, Austria) and $20 \mathrm{mg} / \mathrm{kg}$ propofol (PropofolTM amp, Fresenius Kabi, Austria). Under anaesthesia, in prone position the animal was scanned by a 16-slice computed tomography system (Aquilion, Toshiba Medical Systems). The imaging parameters were as follows: $16 ? 0.5 \mathrm{~mm}$ collimation; $1.0-\mathrm{mm}$ slice thickness, and $1.0-\mathrm{mm}$ reconstruction interval. Dose and scanning parameters have been performed by radiologists in School of Medicine, Atatürk University, Erzurum, Turkey, on the basis of the standardized protocol which considers the documented scanning practices and 
the recent studies to generate optimum image quality while maintaining individual radiation exposure at the lowest level. The coronal images obtained were transfered to a personal computer to perform surface-rendered reconstructions by using software SurfDriver 3.5.6. Those models are processed further to form VRML form in the Cinema 4D software. VRML form enabled us to transport, share and use those data on the personal computers without big computer terminals. Those data were used in the training of the students, researchers, and surgeons on the web.

Keywords: Rabbit, three-dimensional reconstruction, virtual reality modelling language, VRML

\section{P-7}

\section{Morphometry of distal femur}

Cinar S*, Tokpınar A**, Ozcelik O***, Ertekin T*, Nisari $\mathrm{M}^{* *}$

*First and Emergency Aid, Ivrindi Vocational School of Health Service, Balıkesir University Balikesir, Turkey; ${ }^{*}$ Department of Anatomy, Faculty of Medicine, Erciyes University, Kayseri, Turkey; ***Semra and Vefa Kucuk College of Health, Nevsehir Haci Veli Bektas University, Nevsehir, Turkey

Morphology of the distal femur assists elucidate the causes of osteoarthritis, the outcome of osteotomy for osteoarthritisa and the optimal design and procedure for total knee arthroplasty. In this study, morphometric evaluation was performed on 20 right and 20 left femurs which are selected from bone collection at the Erciyes University, Faculty of Medicine, Department of Anatomy laboratory. Measurements of distal femur include the following parameters; the height and width of intercondylar notch, medial condylar and lateral condylar and patellar groove angle. For morphometric evaluation, picture of dry bone samples took and measurements were performed using computerized imagej program. The obtained data were analyzed using SPSS 21 software package. The mean values of height and width of right and left femurs for fossa intercondylaris $2.57 \pm 0.23 \mathrm{~cm}-1.86 \pm 0.29 \mathrm{~cm}$ and $2.56 \pm$ $0.31 \mathrm{~cm}-1.81 \pm 0.26 \mathrm{~cm}$, for lateral condylar $5.41 \pm 0.35 \mathrm{~cm}-2.19 \pm$ $0.26 \mathrm{~cm}$ and $5.55 \pm 0.39 \mathrm{~cm}-2.09 \pm 0.25 \mathrm{~cm}$, for medial condylar $5.20 \pm 0.37 \mathrm{~cm}-2.06 \pm 0.32 \mathrm{~cm}$ and $5.34 \pm 0.37 \mathrm{~cm}-2.10 \pm 0.31 \mathrm{~cm}$ respectively. The mean patellar groove angle of right femurs was $151.23^{\circ} \pm 7.98$ and left femurs was $147.93^{\circ} \pm 7.18$. When the measurements, taken from right and left femur, compared, no statistically significant differences was determined. In addition correlation of patellar groove angle measurement and the other parameters were investigated and we did not determine any correlation. We believe that to know of the values obtained in our study could be useful to other anthropometric studies and anatomic evaluation of the distal femur region before the orthopedic operations

Keywords: Femur, morphometry, ImageJ

\section{P-8}

The morphometric measurements of the lateral sacral mass on sacrum

$\underline{\text { Polat Koc T* }}$, Ertekin $\mathrm{T}^{*}$, Acer $\mathrm{N}^{*}$, Cinar $\mathrm{S}^{* *}$

*Department of Anatomy, Faculty of Medicine, Erciyes University, Kayseri, Turkey; **/vrindi Health Services Vocational School, Balikesir University, Balikesir, Turkey
Sacrum is an important bone structure which is used in the treatment of deformities and injuries of the lumbosacral, sacral, and sacroiliac joints. Thus, if the normal anatomic structure and morphometric values of the sacrum is well known, possible complications which may arise during the operations to be applied to this area can be prevented. The aim of the current study was to make detailed morphometric measurements of pars lateralis and to calculate surface area of the joint on dry sacrum samples. This study was performed by using a digital caliper sensitive to $0.01 \mathrm{mil}$ limeter $(\mathrm{mm})$ on 30 dry sacrum samples obtained from Department of Anatomy in Erciyes University. The age and gender discrimination was not made on dry bone specimens. The auricularis face area of sacrum was calculated, by using ImageJ software. No significant difference was determined between all bilateral morphometric measurements of sacrum $(p>0.05)$. The mean area of the right and left auricularis surface were calculated as $1028.15 \pm 232.92 \mathrm{~mm}^{2}, 1042.45 \pm 220.72 \mathrm{~mm}^{2}$ respectively. We believe that the results of this study will be beneficial for surgeons and clinicians for the surgical approaches to sacrum especially in sacral instrumentation.

Keywords: Sacrum, morphometry, joint, ImageJ

\section{P-9}

\section{The morphometric survey of odontoid process in Turkish population}

Yilmaz MY*, Cakilli M*, Ustun O*, Yilmazer OS*, Gunes $\mathrm{S}^{*}$, Yavuz $Y^{* *}, \underline{\text { Sen Esmer T*** }}$

*Student of Medical School (Term 3), Faculty of Medicine, Ankara University, Ankara, Turkey; **Department of Biostatistics, Faculty of Medicine, Ankara University, Ankara, Turkey; ***Department of Anatomy, Faculty of Medicine, Ankara University, Ankara, Turkey

Objectives: Our aim is to investigate the morphometry of odontoid process in Turkish population and compare it with the literature.

Methods: We studied on 55 dry axis in Ankara University, Department of Anatomy. Minimum anteroposterior and transvers diameter of odontoid process (ADOP and TDOP), anterior and posterior height of odontoid process (AHOP and PHOP), anterior and posterior height of body of axis (AHBA and PHBA) and circumference of neck of odontoid process (CNOP) were measured with digital caliper.

Results: All measurements were recorded. The ossification of cruciate ligament was found in $18.18 \%$ (10 axis) of all bones. In $58.18 \%$ of axis, anterior odontoid fixation was convenient for the fixation of odontoid fracture.

Keywords: Axis, odontoid process

\section{P-10}

Morphometric effects of mianserin treatment on liver tissue in streptozotocin-induced diabetes model

Corumlu U*, Demir Ozkay U**, Ulupinar E*

*Department of Anatomy, Faculty of Medicine, Eskisehir Osmangazi University, Eskisehir, Turkey; **Department of Pharmacology, Faculty of Pharmacy, Anadolu University, Eskisehir, Turkey 
Diabetes Mellitus (DM) is a chronic disorder characterized mainly by hyperglycemia, but also altered metabolism of lipids, carbohydrates and proteins. Emotional reactions, adaptation problems, cognitive complaints and depression are the most common psychiatric conditions in the diabetic patients. Mianserin is a tetracyclic structured, third generation atypical antidepressant drug having noradrenergic and serotonergic effects. It has been reported that $5 \%$ of the cases have liver damage as a consequence of the anti-depressant medication and toxicity caused by over doses plays an important role in these injuries. In this study, whether any change takes place in the liver tissue of diabetic rats treated with different doses of Mianserin was investigated by using histomorphometric methods. Wistar male rats and diabetic rats applied a single dose of streptozotocin were treated with $30 \mathrm{mg} / \mathrm{kg}$ and $45 \mathrm{mg} / \mathrm{kg}$ dosage of Mianserin daily. Following a 2-week treatment period, rats were perfused and 4 İm-thick sections taken from livers were stained with hematoxylin-eosin. Mean diameter of the central veins and sinusoids-to-parenchyma ratios of the liver were estimated by morphometric methods. Statistical analyzes of the data were performed by using one-way analysis of variance followed by Bonferroni multiple comparison tests. Mean diameters of the central veins were not significantly different between healthy controls and diabetic animals after treatment with different doses of Mianserin. The ratio of the sinusoids to liver parenchyma was $4.314 \pm 1.22$ in the control group, whereas it was $3.092 \pm 0.52$ in the diabetic group. After treatment with Mianserin, this ratio was similar $(4.640 \pm 0.45$ and $3.394 \pm 0.37$ for 30 and $45 \mathrm{mg} / \mathrm{kg}$, respectively). These results have shown that acute Mianserin treatment has no hepatotoxic effect on the liver sinusoids in streptozotocin induced experimental diabetic model. In the future studies, it is planned to compare the chronic and high dosage effects of this drug.

Keywords: Diabetes mellitus, mianserin, liver injury

\section{P-11}

The evaluation of the protective effect of intravenous lipid emulsion treatment on liver in diazinon intoxication

Tas $U^{*}$, Ayan $M^{* *}$, Uysal $M^{*}$, Esen $M^{* *}$, Meydan $S^{* * *}$, Cicek $M^{*}$, Sarsilmaz $M^{* * *}$

*Department of Anatomy, Faculty of Medicine, Gaziosmanpasa University, Tokat, Turkey; **Department of Emergency Medicine, Faculty of Medicine, Gaziosmanpasa University, Tokat, Turkey; $* * *$ Department of Anatomy, Faculty of Medicine, Sifa University, Izmir, Turkey

Objectives: Organophosphate compounds currently substance used to kill insects are very toxic materials. Several organophosphates including diazinon can be ingested or inhaled, or absorbed through the skin but it may be as a maximum of acute toxicity when administered by oral intake. Intravenous lipid emulsion (ILE) treatment is used as a new treatment method in cases of systemic toxicity caused by local anesthetics. This study aimed to research the positive effect of intravenous lipid emulsion on rat liver tissue in the toxication model of diazinon.
Methods: 21 male Wistar albino rats, (weighing 180-200 g) randomly divided into three equal groups. Groups organized as; Group I control, Group II diazinon, Group III diazinon + lipid emulsion treatment. Group I, only $1 \mathrm{ml}$ corn oil was given by gavage. Group II, $335 \mathrm{mg} / \mathrm{kg}$ diazinon were given by gavage. Group III, in addition to diazinon $20 \%$ lipid solution (3 $\mathrm{ml} / \mathrm{kg}$ ) were administered via tail vein into rats. Animals were killed by exsanguination under ketamine/xylasine anesthesia, and liver tissues removed. After routine histological procedures, 5 micrometer histological sections were taken by rotary microtome. Tissue sections were examined for apoptotic changes by Bax, Caspase-3 and TUNEL immunohistochemical staining.

Results: An increase of apoptotic cell death and formation of degenerative changes occurred in the diazinon group. Additionally, a decrease apoptosis was observed in the diazinon + lipid emulsion treatment group when compared with the diasinon group.

Conclusion: The findings from our study suggest that lipid emulsion treatment has protective efficacy on the diazinoninduced liver toxicity.

Keywords: Diazinon, toxicity, apoptosis, liver

\section{P-12}

\section{Effects of aging on interververtebral disc tissue}

$\underline{\text { Tas } \mathrm{U}^{*}}$, Uysal $\mathrm{M}^{*}$, Aytekin $\mathrm{K}^{* *}$, Bicakci $\mathrm{H}^{* * *}$, Acikgoz $\mathrm{R}^{* * *}$, Ozyurt B*, Sarsilmaz $M^{* * *}$

*Department of Anatomy, Faculty of Medicine, Gaziosmanpasa University, Tokat, Turkey; ${ }^{*}$ Department of Orthopaedic, Tokat State Hospital, Tokat, Turkey; ***Sifa University, Faculty of Medicine, Department of Anatomy, Izmir, Turkey

Objectives: In our study we aimed to demonstrate the number of chondrocytes in rat lumber discs from both young and aged animals using by stereological methods. A lot of study has demonstrated that the intervertebral disc is an avascular tissue element contains cells and extensive extracellular matrix. The annulus fibrosus is predominantly collagenous; the matrix of the central nucleus pulposus is rich in proteoglycans. Intervertebral discs go through age-related degenerative changes. The annulus fibrosus becomes weaker with age and, the cartilage end plates begin weakening, clefts begin to form, and there is sclerosis of the subchondral bone.

Methods: Twenty Wistar-albino rats were included in the study. The rats were separated into two groups: 2-month-old rats $(\mathrm{n}=10)$ as the young group, 18 -month-old rats $(\mathrm{n}=10)$ as the old group. The intervertebral disc tissues obtained from the lumbar spine (L1-L4, 4 discs) were used for stereological analysis.

Results: In our study we demonstrated decreased number of chondrocytes in aging rats when the compared to the young.

Conclusion: This study demonstrated that the aging process lead to decrease the number of chondrocytes in inter vertebral disc.

Keywords: Intervertebral disc, aging, stereology 


\section{P-13}

Dermatoglyphic features in migraine

$\underline{\text { Sabanciogulları } V^{*}}$, Cevik $S^{* *}$, Erdal $M^{* * *}$, Bolayir $E^{* *}$, Koşar MI****

*Department of Anatomy, Faculty of Medicine, Cumhuriyet University, Sivas, Turkey; ${ }^{*}$ Department of Neurology, Faculty of Medicine, Cumhuriyet University, Sivas, Turkey; ${ }^{* *}$ Kayseri Women and Child Hospital, Kayseri, Turkey; $* * * *$ Department of Anatomy, Faculty of Medicine, Mugla Sitki Kocman University, Mugla, Turkey

Migraine is a common headache disorder. Migraine is observed $18 \%$ in women, while $6 \%$ in men. Especially, decline in quality of life and can lead to serious work force loss in individuals aged 30-49. Although the exact cause of the disease is unknown, environmental factors and genetic predisposition are accused in etiology. Dermatoglyphics are specific patterns formed by coming together of epidermal ridges. They are formed in intrauterine period and remain unchanged a lifelong. However, some disorders occurred depending on genetic predisposition might cause deteriorations in pattern and number of dermatoglyphics. Thus, the fingertip and palm dermatoglyphic samples of patients diagnosed with multiple sclerosis were compared with the control group and results were presented with literature. Dermatoglyphics data obtained from 51 patients and 70 healthy controls with digital scanner were transferred to a computer. Image J program was used and atd, dat, adt angles, a-b ridge count, sample types of all fingers and ridge counts were calculated. The obtained data was analyzed by SPSS 15.0 software. In statistical evaluation, t-test, Mann-Whitney $U$ and chi-square test were used. In both hands of the patients with migraine, $a-b$ ridge count, ridge counts in all fingers and total ridge counts increased. This increase was statistically significant except for the right hand little finger. Furthermore, atd angle was higher in a statistically significant on both hands of patients with migraine compared to healthy controls. Deviations from normality in the distribution of dermatoglyphic samples supports the accused genetic predisposition in the migraine etiology. However, further studies are needed to be able to identify individuals with congenital predisposition to migraine.

Keywords: Migraine, etiology, dermatoglyphics

\section{P-14}

\section{The historical adventure of anatomy of the heart}

Caglar $\mathrm{V}^{*}$, Celik N**, Sevkioglu B*

*Department of Anatomy, Faculty of Medicine, Namik Kemal University, Tekirdag, Turkey; **Department of Medical History and Ethics, Faculty of Medicine, Namık Kemal University, Tekirdag, Turkey

Medicine has existed with humanity and it is an indispensable element of human life. Throughout history, the heart has been believed to possess an important function in the body. Our aim historical development of the heart is evaluate in terms of the anatomical developments. In the ancient civilizations, it was demonstrated that there were both blood and the air in the vessels in the body. Moreover, the heart beats have been mentioned. In ancient Greece, it was identified that the arteries and veins had different functions. In Alexandria Medical School were discussed the tricuspid valve and its functions. According to Galen some blood from the right ventricle to the left ventricle transfers via pores found in the septum. This theory domiated Western medicine until 17 th centruy due to "strict obedience to authority". Avicenna, as the successor of Galen has been recognized. Ibn al-Nafis unlike Galen and Avicenna clearly described that blood came from the right heart to the lungs and mixed there with air and then returned to the left heart. Ibn al-Nafis discovery of pulmonary circulation in 13th centruy. In the historical process, the first study subject were about anatomical and physiological structures of the heart. Then the process have included all stages form determination of blood groups to a heart transplant.

Keywords: Anatomy of heart, Ibn al-Nafis, Galen, Avicenna

\section{P-15}

\section{Morphological comparison of solitary and normal kidney on ct images}

Caglar $\mathrm{V}^{*}$, Kurt $\mathrm{O}^{* *}$, Uygur $\mathrm{R}^{*}$, Sener $\mathrm{U}^{* * *}$, Ozcaglayan $\mathrm{O}^{* * *}$, Kasirga $\mathrm{Z}^{*}$, Tugtag $\mathrm{B}^{*}$

*Department of Anatomy, Faculty of Medicine, Namik Kemal University, Tekirdag, Turkey; **Department of Urology, Faculty of Medicine, Namik Kemal University, Tekirdag Turkey; ${ }^{* *}$ Department of Physiology, Faculty of Medicine, Namik Kemal University, Tekirdag, Turkey; $* * *$ Department of Radiology, Faculty of Medicine, Namik Kemal University, Tekirdag, Turkey

Objectives: This study was undertaken to compare the morphological differences between individuals with one functioning kidney and individuals with two functioning kidneys through the measurement of dimensions and stereological renal volume estimations.

Methods: The target sample consisted of patients who underwent an abdominal CT examination after attending Namik Kemal University's Clinical Research and Application Center seeking medical attention. The volumetric estimations of CT images were based on the Cavalieri Principle.

Results: Of the overall study population, 22 had one healthy kidney and 30 had two healthy kidneys. Average renal dimensions were volume $159 \mathrm{~cm}^{3}$, length $10.7 \mathrm{~cm}$, width $6.6 \mathrm{~cm}$, and thickness $5 \mathrm{~cm}$ among anatomically normal individuals. The corresponding figures in the sole-functioning kidney group were volume $274 \mathrm{~cm}^{3}$, length $12 \mathrm{~cm}$, width $7 \mathrm{~cm}$, and thickness $6 \mathrm{~cm}$.

Conclusions: Sole-functioning kidneys were longer, thicker, wider, and more voluminous compared with normal kidneys. We believe that the morphological differences between solitary and normal kidneys observed in this study in terms of dimensions and volume may represent a contribution to the existing literature that may assist in accurate volume and dimension estimations for clinical diagnosis and treatment of renal conditions.

Keywords: Kidney volume, kidney length, CT, Cavalieri principle 


\section{P-16}

The importance of fetal ultrasonographic parameters for anatomical study

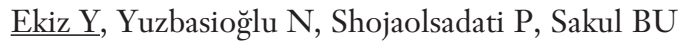

Department of Anatomy, Faculty of Medicine, Istanbul Medipol University, Istanbul, Turkey

Ultrasonography (USG) is widely used especially in determination of fetal gestational age detection of congenital anomalies, and evaluation of fetal growth and development. Fetus is visible and measurable by USG, assessing the fetus functionally could be possible by morphometric evaluation of fetal morphology and development. Any maternal and fetal harmful effect of sonography has not been detected, because of this property it is recommended that USG should be used during certain periods of pregnancy used as a screening and diagnostic method. USG is used for estimation of gestational age and assessing fetal growth and development by using various parameters such as crown-rump lenght (CRL), head circumference (HC), femur length (FL), foot length (fl), biparietal diameter (BDP), abdominal circumference (AC) and nasal bone lenght (NL). For example, CRL is a parameter that is used during the first trimester. $\mathrm{HC}, \mathrm{FL}$ and $\mathrm{fl}$ are parameters used in the second and third trimesters. In addition, it was revealed that USG data of living fetuses and data from aborted fetuses were also found to be similar. It is important to know the normal and pathological values of fetal parameters by using both the direct measurement of anatomical dissection and USG studies. Because data obtained from certain periods of pregnancy is important to evaluate the developmental anatomical features of the fetus and detect any congenital and developmental disorder risk. In this paper, it was aimed to evaluate the importance of USG parameters which are routinely assessed and some anatomical parameters recommended in recent papers in term of anatomical and ultrasonografic studies and also monitoring the fetal development.

Keywords: Anatomy, fetal development, fetal period, gestation, ultrasonography

\section{P-17}

The relationship of fossa cranii posterior, cerebrum and cerebellum morphometry with tonsillar herniation

Tastemur $\mathrm{Y}^{*}$, Sabanciogulları $\mathrm{V}^{*}$, Salk I**, Sonmez $\mathrm{M}^{*}$, Cimen $M^{*}$

*Department of Anatomy, Faculty of Medicine, Cumhuriyet University Sivas, Turkey; **Department of Radiology, Faculty of Medicine, Cumhuriyet University, Sivas, Turkey

Tonsillar herniation is a condition which is manifested as herniation of the brain parts originated from hindbrain through foramen magnum to vertebral canal. Although etiology of tonsillar herniation is unclear, it is been suggested that it may be related to congenital or acquired reasons. Especially it is been speculated that primer mesodermal insufficiency may effect the size of fossa cranii posterior. So that reason we aimed to perform measurements on cranium, cerebrum and cerebellum and to enlighten tonsillar herniation etiology. The magnetic resonance images of 1052 patients (629 female and 423 male) who have no disease which can effect bones. The mean age was $36.58 \pm 22.34$ (1-94 age). Measurements were performed by using T1, T2 midsagittal and axial images. While nine parameter have been used to evaluate cranium morfometry, another nine parameter have been use to evaluate cerebrum and cerebellum morfometry. Collected data has been analyzed in SPSS (14.0) statistic software by using t-test and Mann-Whitney U test. The significance level was set at 0.05 . In individuals with tonsillar herniation, while front-back diameter of foramen magnum, cerebellum height and sagittal diameter of cerebellum were increased, maximum cranial height, supraocciput length, clivus length and the height of fossa cranii posterior were decreased. Also in all age groups, there was no statistically difference between healthy control and the people with tonsillar herniation in terms of tentorial slope angle. Mean herniation value was $4.85 \pm 3.09 \mathrm{~mm}$ in people with tonsillar herniation. Our results about cranium morfometry support the theory in which it is been speculated that hypoplastic posterior cranial fossa due to mesodermal insufficiency may be a factor play role in tonsillar herniation etiology.

Keywords: Tonsillar herniation, posterior cranial fossa, magnetic resonance imaging, cerebral and cerebellar morfometry

\section{P-18}

Assessment of musculoskeletal system knowledge and skills of the medical students

Ulasli AM*, Yaman F*, Gonul Y**, Gokalp H**, Ozen MT ${ }^{* * *}$, Ruzgar $\mathrm{H}^{* * *}$, Gulsari $\mathrm{Y}^{* *}$, Dundar $\mathrm{U}^{*}$

*Department of Physical Medicine and Rehabilitation, Faculty of Medicine, Afyon Kocatepe University, Afyonkarahisar, Turkey; **Department of Anatomy, Faculty of Medicine, Afyon Kocatepe University, Afyonkarahisar, Turkey; ***3rd Grade Students, Faculty of Medicine, Afyon Kocatepe University, Afyonkarahisar, Turkey

Objectives: Musculoskeletal system (MSS) knowledge and clinical skills have been shown to be an area of weakness in medical education and practitioners, and neglected in daily clinical practice. The purpose of this study was to evaluate and compare MSS knowledge and skills of the third and sixth year medical students.

Methods: The study included 41 volunteers from third year and 35 volunteers from sixth year medical students. Participants were asked to mark long head of biceps brachii muscle tendon (BTLH), lateral epicondyle (LE), greater trochanter (GT) and medial knee joint space $(\mathrm{MK})$ areas were with physical examination. After students marked the choosen anatomical landmarks one by one on the same subject, the accuracy of the results were checked with ultrasonography.

Results: Total success rate was $51.8 \%$ in third year students while it was $68.6 \%$ in sixth year students and the difference between two groups was statistically significant $(p=0.004)$. Students performance to locate LE and GT was high but they were failed to locate BTLH and MK. 
Conclusion: This study demonstrated that medical students improved their MSS skills that they obtained in preclinical education period, during clinical education period. Nevertheless this improvement was not adequate. Regarding increasing number of patients admitting to hospitals with the complaints of MSS, medical faculties should focus on improving MSS clinical skills teaching and develope effective strategies to meet this requirements.

This research is puplished in Kocatepe Medical Journal: A. M. Ulaşli, F. Yaman, Y. Gönül, H. Gökalp, M. T. Özen, H. Rüzgar, Y. Gülsarı, and Ü. Dündar, "Assessment of Musculoskeletal System Knowledge and Skills of the Medical Students," vol. 15, no. 2, pp. 147-151, 2014.

Keywords: Medical education, musculoskeletal system, anatomy

\section{P-19}

Anti-inflammatory and antiapoptotic effect of interleukine-18 binding protein on the spinal cord ischemia-reperfusion injury

Karavelioglu E*, Gonul $\mathrm{Y}^{* *}$, Kokulu $\mathrm{S}^{* * *}$, Hazman Ö̈***, Bozkurt $\mathrm{F}^{* * * * *}$, Koçak $\mathrm{A}^{* * * * *}$, Eser $\mathrm{O}^{* * * * * * *}$

*Department of Neurosurgery, Faculty of Medicine, Afyon Kocatepe University, Afyonkarahisar, Turkey; ${ }^{*}$ Department of Anatomy, Faculty of Medicine, Afyon Kocatepe University, Afyonkarahisar, Turkey; $* * *$ Department of Anesthesia and Reanimation, Faculty of Medicine, Afyon Kocatepe University, Afyonkarahisar, Turkey; $* * * *$ Department of Chemistry, Faculty of Science and Arts, Afyon Kocatepe University,

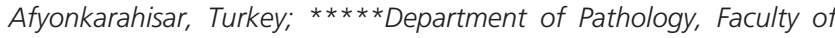
Veterinary, Afyon Kocatepe University, Afyonkarahisar, Turkey; $* * * * * \star$ Department of Histology, Faculty of Medicine, Dumlupinar University, Kutahya, Turkey; $* * * * * * \star$ Department of Neurosurgery, Faculty of Medicine, Balikesir University, Balikesir, Turkey

Objectives: The aim of this study is to investigate the probable anti-inflammatuary and anti-apoptotic effect of Interleukine-18 binding protein (IL-18BP) on spinal cord ischemia reperfusion (I/R) injury in rats.

Methods: Twenty one adult male Wistar-Albino rats, weighing 250-300 g (mean $270 \mathrm{~g}$ ) were divided randomly into three groups of seven rats as: Group 1: Sham (n: 7) only laparatomy, Group 2: I/R (n: 7), Group 3: I/R and IL-18BP $100 \mu \mathrm{g} / \mathrm{kg}$ (n:7). Proinflammatory cytokines IL-1 $\beta$, IL-6, IL-18, TNF- $\alpha$ and IFN- $\gamma$ levels were determined by ELISA method in the blood samples of rats. Apoptosis was evaluated immunohistochemically using apoptotic marker caspase 3 in the spinal cord tissue samples.

Results: Both IL-18 and TNF- $\alpha$ levels were significiantly decreased in the IL-18BP group compared to the sham group. The highest caspase 3 scores were in I/R group while the lowest scores were in sham group. The mean Tarlov score of the I/R group was significiantly lower than the sham group. However, the mean Tarlov score of the IL-18BP group was significantly higher than the I/R group.

Conclusion: IL-18BP has anti-inflammatuary efficiency on spinal cord I/R injury. The immunohistopatological result of the present study also demonstrated that IL-18BP has anti- apoptotic effect on spinal cord I/R injury. IL-18BP was shown to be effective in spinal cord ischemia/reperfusion model.

This research is puplished in Inflammation indexed in (SCI)science citation index: Karavelioğlu E, Gönül Y, Kokulu S, Hazman O, Bozkurt F, Koçak A, Eser O. Anti-inflammatory and Antiapoptotic Effect of Interleukine-18 Binding Protein on the Spinal Cord Ischemia-Reperfusion Injury. Inflammation. 2014 Jun;37(3):917-23.

Keywords: Interleukine-18 binding protein, spinal cord, ischemia reperfusion, cytokines, caspase-3

\section{P-20}

\section{Cervical vertebra osteophytes causing dysphagia: two case reports}

Fazliogulları $Z^{*}$, Nayman $\mathrm{A}^{* *}$, Kibar $\mathrm{E}^{* * *}$, Karabagli $\mathrm{H}^{* * * *}$, Ozturk K*** Karabulut AK*

*Department of Anatomy, Medical Faculty, Selcuk University, Konya, Turkey; **Department of Radiology, Medical Faculty, Selcuk University, Konya, Turkey; ${ }^{* \star *}$ Department of Otolaryngology, Medical Faculty, Selcuk University, Konya, Turkey; $* * * \star$ Department of Neurosurgery, Medical Faculty, Selcuk University, Konya, Turkey

The osteophytes which are elongated from anterior part of the vertebral body to the pharynx and esophagus can cause symptoms such as dysphagia, odynophagia, otalgia or foreign body sensation in the throat. The rare cause of the dysphagia is the pressure of these osteophytes on the posterior pharyngeal wall and esophagus, and it is commonly seen at middle age and elderly. The incidence of the asymptomatic anterior cervical vertebral osteophytes is $20-30 \%$. It can be symptomatic when enlarged and it is rarely reported in literature. While large osteophytes can cause dysphagia by simple mechanic effect, small osteophytes can be effective at the level of the cricoid cartilage. Esophagus is anatomically fixed at the levels of the cricoid cartilage and the diaphragm. Remainder part of the esophagus is more flexible. Pathophysiologically, esophagus is more exposed to the pressure of the cervical vertebra body at the cricoid cartilage. Therefore the small osteophytes at the cricoid region even can cause dysphagia. Two cases, who consulted for complaints such as swallowing difficulty, sensation of sting and sore and coughing during feeding, were assessed to evaluate swallowing function using flexible laryngoscopy after physical examination in otolaryngology clinic at our faculty. It is identified some fixed mass on the posterior wall of the hypopharynx and the superior part of the esophagus. Some osteophyte structures, which pressure on to the posterior esophagus from the corpus vertebra at the level of the C3-4, were observed in radiological examination. Due to continuing patients' complaint after medical treatment, surgery was performed in collaboration with neurosurgery clinic. After surgery, it is observed that both patients' complaints were disappeared in the long term follow-up. In addition to these complaints, cervical osteophytes can be rarely caused some problems by obstructing intubation during anesthesia too and it should be considered that it can be encountered this type of respiratory problems in emergencies.

Keywords: Cervical osteophytes, dysphagia, esophagus. 


\section{P-21}

Case report: the right vertebral artery originated from the right occipital artery and absence of transverse foraminae

Oner $Z^{*}$, Oner $S^{* *}$, Sagir Kahraman $A^{* * *}$

*Department of Anatomy, Faculty of Medicine, Inonu University, Malatya, Turkey; ${ }^{* *}$ Department of Radiology, Teaching and Research Hospital, Karabuk University, Karabuk, Turkey; $* * \star$ Department of Radiology, Faculty of Medicine, Inonu University, Malatya, Turkey

Variations in the origin of the vertebral artery are congenital anomalies that occur during the embryological development. Multiple variations related to vertebral artery origin have been reported in the literature. Abnormal vertebral artery origin usually is determined as incidental findings during angiographic or postmortem anatomical studies. Although most of the cases are asymptomatic, in patients with vertebral artery anomaly has been described symptoms such as dizziness. The anomalous origin of the right vertebral artery is rare and separated three categories: 1. Originated from the aorta, 2. Originated from the carotid arteries, 3. Duplicated origin. In this case, we aimed to present the right vertebral artery originated from the right occipital artery and concomitant anomalies of the transverse foramina that not been reported previously in the literature.

Case report: In a 32 year old female patient followed in our hospital because of vertigo, right vertebral artery was not observed on MRI and then CT angiography examination was performed. CT angiography showed hypoplasia of the right transverse foramina in the levels of $\mathrm{C} 1, \mathrm{C} 5, \mathrm{C} 6$ vertebra and aplasia of the right transverse foramina in the levels of $\mathrm{C} 2, \mathrm{C} 3, \mathrm{C} 4$ vertebra. The right vertebral artery originated from the right occipital artery continues to its normal course by entering to the cranium through the foramen magnum at the level of atlantooccipital junction.

Keywords: Anomalous origin, right vertebral artery, occipital artery, CT angiography

\section{P-22}

The beneficial effects of $\mathrm{N}$-acetyl-cysteine, amifostine and ascorbic acid in methotrexateinduced lung injury

Elbe $H^{*}$, Akbulut AS**, Dogan $Z^{* * *}$, Kaymaz T ${ }^{* * * *}$, Turkoz $\mathrm{Y}^{* * * * *}$

*Department of Histology and Embryology, Faculty of Medicine, Inonu University, Malatya, Turkey; **Department of General Surgery, Faculty of Medicine, Inonu University, Malatya, Turkey; $* * *$ Department of Anatomy, Faculty of Medicine, Adiyaman University, Adiyaman, Turkey; $\star * \star *$ Department of Molecular Biology and Genetics, Faculty of Sciences and Arts, Inonu University, Malatya, Turkey; $* * * * *$ Department of Biochemistry, Medical Faculty Inonu University, Malatya Turkey

Objectives: In this study, we aimed to evaluate that beneficial effects of N-acetyl-cysteine (NAC), amifostine (AMF) and ascorbic acid (ASC) in methotrexate (MTX)-induced lung injury.
Methods: 55 Sprague Dawley rats were divided into 5 groups. Control, MTX (20 mg/kg/single dose/ip, was carried out 2 nd day of experiment), MTX+NAC (50 mg/kg/day/po, 7 days), MTX+AMF $(50 \mathrm{mg} / \mathrm{kg} /$ day/ip, 7 days), MTX+ASC (10 $\mathrm{mg} / \mathrm{kg} /$ day/ip, 7 days). At the end of the study lungs were removed and routine histological procedures were done. Hematoxylin and eosin and Masson's trichrome-stained sections were evaluated and histopathological damage score (infiltration, hemorrhage, alveolar congestion, fibrosis) was calculated (0-3, maximum score $=12)$. Tissue malondialdehyde (MDA) and glutathione (GSH) levels were measured.

Results: Control group was normal in histological appearance. In MTX group, infiltration, hemorrhage, alveolar congestion and fibrosis were observed. The mean damage score was $8.62 \pm 0.49$ in MTX group. In treatment groups, histopathological changes were reduced. The mean damage score was $4.62 \pm 0.32$ in MTX+NAC group, $5.12 \pm 0.35$ in MTX $+\mathrm{AMF}$ group and $4.87 \pm 0.39$ in MTX+ASC group. When compared with the MTX group, significant differences were detected in treatment groups ( $\mathrm{p}=0.001$, for all). In MTX group, an increase in MDA levels and a decrease in GSH levels were identified when compared with the control group $(\mathrm{p}<0.05)$. In treatment groups, a decrease in MDA levels and an increase in GSH levels when compared with the MTX group $(\mathrm{p}<0.05)$.

Conclusion: We concluded that NAC, AMF and ASC treatments are beneficial in MTX-induced lung injury.

Keywords: Methotrexate, N-acetyl-cysteine, amifostine, ascorbic acid, lung injury

\section{P-23}

The effects of $\mathrm{N}$-acetyl-cysteine, amifostine and ascorbic acid on methotrexate-induced cerebrum and cerebellum damage: a comparative study

$\underline{\text { Dogan } Z^{*}}$, Akbulut AS ${ }^{* *}$, Elbe $\mathrm{H}^{* * *}$, Erdemli ME****, Turkoz $\mathrm{Y}^{* * * *}$

*Department of Anatomy, Faculty of Medicine, Adiyaman University, Adiyaman, Turkey; **Department of General Surgery, Faculty of Medicine, Inonu University, Malatya, Turkey; $* * *$ Department of Histology and Embryology, Faculty of Medicine, Inonu University, Malatya, Turkey; ${ }^{* * *}$ Department of Biochemistry, Faculty of Medicine, Inonu University, Malatya, Turkey

Objectives: In this study, we aimed to evaluate that the effects of $\mathrm{N}$-acetyl-cysteine (NAC), amifostine (AMF) and ascorbic acid (ASC) on methotrexate (MTX)-induced cerebrum and cerebellum damage.

Methods: 55 Sprague Dawley rats divided into 5 equal groups. Control, MTX $(20 \mathrm{mg} / \mathrm{kg} /$ single dose/ip, was carried out 2 nd day of experiment), MTX+NAC (50 mg/kg/day/po, 7 days), MTX+AMF $(50 \mathrm{mg} / \mathrm{kg} /$ day/ip, 7 days $)$, MTX+ASC $(10$ $\mathrm{mg} / \mathrm{kg} /$ day/ip, 7 days). At the end of the study cerebrum and cerebellum were removed and routin histological procedures were done. H-E-stained sections were evaluated and histopathological damage score (For cerebrum; perivascular edema, infiltration, congestion, hemorrhage, vacuolization) (For cerebellum; 
loss of purkinje cell, purkinje cell degeneration, hemorrhage) was calculated (0-3). Sections were examined using a Leica DFC280 light microscope and a Leica Q Win Image Analysis system (Leica Micros Imaging Solutions Ltd., Cambridge, UK). Statistical analysis was carried out using the SPSS 13.0 (SPSS Inc., Chicago, III., USA) statistical program. For comparison Kruskal-Wallis and Mann-Whitney U tests were used. All data are expressed as arithmetic mean \pm SE. $\mathrm{p}<0.05$ was regarded as significant.

Results: Cerebrum and cerebbelum tissues of control group was normal in histological appearance. In cerebrum-MTX group, perivascular edema, infiltration, congestion, hemorrhage, neuronal vacuolization and cerebellum-MTX group, loss of purkinje cell, purkinje cell degeneration, hemorrhage were observed. In treatment groups, histopathological changes were reduced. When compared with the MTX group, significant differences were detected in treatment groups $(\mathrm{p}<0.05$, for all tissues).

Conclusion: We concluded that NAC, AMF and ASC treatments are beneficial in MTX-induced cerebrum and cerebellum damage in rats.

Keywords: Methotrexate, N-acetyl-cysteine, amifostine, ascorbic acid, lung injury

\section{P-24}

Pilot study: the evaluation of physicians opinions about given anatomy education in faculty of medicine

Cuglan $\mathrm{S}^{*}$, Irmak Sapmaz $\mathrm{H}^{*}$, Y1lmaz N*, Tekin $\mathrm{C}^{* *}$, Colak $\mathrm{C}^{* * *}$, Ozbag D*

*Department of Anatomy, Faculty of Medicine, Inonu University, Malatya, Turkey; ${ }^{*}$ Department of Public Health, Faculty of Medicine, Inonu University, Malatya, Turkey; ***Department of Biostatistics and Medical Informatics, Faculty of Medicine, Inönü University, Malatya, Turkey

Objectives: In this study, we aimed to assess the opinion of the physicians working in Inonu University Faculty of Medicine about their anatomy education in medical school.

Methods: Twenty physicians were enrolled to study from Inonu University Faculty of Medicine. Participants were questioned with forty-five items in the survey. There were questions about participants demographic characteristics and determining their educational information in the survey. Additionally, there were questions that evaluated their general thoughts and perceptions about anatomy and theoretical and practical lectures of anatomy.

Results: The mean age of the participants was $33,2 \pm 9,1$ in the study. The $45 \%(n=9)$ of the participants was male and $55 \%$ $(n=11)$ was female. The $60 \%(n=12)$ of the participants was research assistants and $40 \%(n=8)$ was a instructor of the faculty. The $65 \%(n=13)$ of the participants was working in internal medicine and $35 \%(n=7)$ was working in basic medical sciences. Fifty percent of physicians were stated that they had used cadaver, bone and maquette together in their laboratory practice. The $25 \%(n=5)$ of the participants stated that not to see any cadavers, $40 \%(n=8)$ of the participants stated that had seen only one cadavers. Eighty percent of participant gave "no" answer to the question of whether choosing the anatomy as a specialist. When asked about reason, $40 \%(n=8)$ percent did not give any answer. Six physicians confessed that they did not prefer anatomy due to requiring a lot of memorization. Eighty percent of participant was stated that basic informations were explained in great detail. It is said that laboratory practices more memorable than theoretical lectures. Seventy percent of participant was concluded that using model instead of cadavers could not be enough.

Conclusion: This was a pilot study. We planned to extend this study with more physicians. We consider that this data would contribute to improve of anatomy education.

Keywords: Faculty of medicine, education of anatomy, physician

\section{P-25 \\ Bilateral pneumatization of the superior turbinates: a case report}

Gun C, Yenigun A, Fazliogullari Z, Unver Dogan N

Department of Anatomy, Faculty of Medicine, Selcuk University, Konya, Turkey

The superior concha is medial process of the ethmoidal labyrinth and describes as a small curved lamina, posterosuperior to the middle concha.It roofs the superior meatus and it is the shortest and shallowest of the tree conchae. The sphenoidal sinus opens to the sphenoethmoidal recess which is above the superior concha. Nasal turbinates humidify, filtration and warm air and arrangement of airflow. Concha bullosa is the name given to pneumatization of the nasal turbinates. The superior nasal concha provides a constant landmark by sphenoid sinus. A 35-year-old, non-smoker man was admitted to our hospital with chronic headache complaint. His pain is anterior part of the head and both of his eyes. Nasal endoscopy revealed that nasal septum was midline and inferior turbinates were hypertrophic. Bilateral pneumatization of the superior turbinates was determinated by computed tomography. Patient was discharged with medical treatment and clinical follow up. Concha bullosa is the most common anatomic variation of the osteomeatalcomplex. It was reported that pneumatization of the superior concha was determinated 44 of the 200 sides (100 patients) for a prevalence of $22 \% .27$ cases had superior concha bullosa; 17 patients had bilaterally and 10 patients had unilaterally. There was a significant association between unilateral or dominant concha and contralateral septal deviation. It was reported that concha bullosa rate in septal deviated patients was 45.34 while rate in non-septal deviated patients was 18.95 . Septal deviation, concha bullosa and Haller cells are the most common structural abnormalities that may cause rhinosinusitis. They obstruct opening of sinuses and that cause of secretion retention, obstructed ventilation and finally rhinosinusitis and polyp formation.

Keywords: Superior concha, concha bullosa, headache 


\section{P-26}

Historic signature in the atlas of anatomy

Donmez D*, Kutoglu T*, Sinav A**

*Department of Anatomy, Faculty of Medicine, Trakya University, Edirne, Turkey; **Faculty of Medicine, Department of Anatomy,

Sanko University, Gaziantep, Turkey

Eduard PERNKOPF lived between the years 1888-1955 was an Austrian anatomist. He created an anatomy atlas. It caused controversy. The reason was controversial majority of dissection studies conducted during holocaust and Eduard PERNKOPF was a member of the National Socialist Party. Almost all the illustrations in the Atlas swastika and SS symbols in the signature section there was. In this context, it was benefited the author who lived at he same time of holocaust. We found some similarities with Sobotta atlas which has been using widely. In Sobotta, (1982 Urban \& Schwarzenberg, Helmut Ferner and Jochen Staubesand edited) had 27 illustrations same with Pernkopf Atlas. Lepier was the head painter of Pernkopf Atlas whose illustrations in Sobotta. Sobotta 1982 Volume 1.43 illustrations of 614 Lepier signed. Sobotta 1982 Volume 2.72 illustrations of 545 Lepier signed. University of commission in 1998, according to the report explained the cadavers from the Gestapo camp had been used in research and teaching. According to allegations, 1380 people were executed in Nazi Camp and their bodies was taken by the University of Vienna and dissected cadavers were used in the Pernkopf atlas. Illustrations not to evidence determine the ethnic origin of cadavers directly, but some minor details raised suspicion. Lepier had drawn an illustration of the head was shaved, there were roughly. Another painter Karl Endtresser had drawn male cadaver which had been circumcised. Atlas regarding the ethical debate, there are several different ideas from scientists. It is clear that Removing the atlas from publishing can't change the past. it is important thing to be aware about priceless studies.

Keywords: Atlas, pernkopf, holocaust

\section{P-27}

\section{Bilateral "S" shaped curled internal carotid artery case}

Aydın Kabakcı AD*, Akın D*, Cicekcibasi AE*, Yilmaz MT*, Keskin $\mathrm{S}^{* *}$

*Department of Anatomy, Meram Medicine Faculty, Necmettin Erbakan University, Konya, Turkey; **Department of Radiology, Meram Medicine Faculty, Necmettin Erbakan University, Konya, Turkey

Objectives: Internal carotid artery arises from common carotid artery from carotid bifurcation in cervical region at $\mathrm{C} 4$ vertebrae level and heads towards the skull base. It supplies blood for brain, eye, forehead and some parts of the nose. Internal carotid artery enters the cranial cavity by the external opening of the carotid canal of the temporal bone. It leaves the canal from the internal opening and proceeds from the roof of foramen lacerum and carotid sulcus and then enters the cavornous sinus. After that it leaves cavernous sinus by piercing dura mater. It also pierces the arachnoid mater and heads back towards the anterior perforated substance region. Internal carotid artery and the arterial branches from vertebral artery forms the Willis polygon by anastomos- ing. And then the blood gets distributed to all parts of the brain by vertebral and carotid systems.

Case report: We have spotted bilateral internal carotid artery variation on a computed tomography images of a 67-year-old patient who applied to Necmettin Erbakan University Meram Medical Faculty Radiology Department for CT-angiography. Cervical part of internal carotid artery was observed not as straight but as curled twice, dispite the classical knowledge.

Results: The firts classification study for cervical part of internal carotid artery had been performed by Weibel and Fields (2000). They had mentioned in their study that internal carotid artery shows 4 kinds of variations as Type 1: Internal carotid artery steering straight, Type 2: Internal carotid artery steering as $S$ or $\mathrm{C}$ shaped and this curling being towards lateral or medial, Type 3: Internal carotid artery being curled once or twice and Type 4: Internal carotid artery forming a spiral on itself. In our case, internal carotid artery was found to be consistent with Type 3.

Conclusion: The course of internal carotid artery in cervical region is highly important for the success of the operations performed on this area. Therefore the knowledge of the morphological differences of the artery should be possessed.

Keywords: Internal carotid artery, curling, variation

\section{P-28}

\section{Morphometric evaluation of foramen magnum in dried human skulls}

Akin $\mathrm{D}^{*}$, Kabakci Aydin $\mathrm{AD}^{*}$, Buyukmumcu $\mathrm{M}^{*}$, Sindel $\mathrm{M}^{* *}$, Ogut $\mathrm{E}^{* *}$, Yilmaz MT*, Sahin $\mathrm{G}^{*}$

*Department of Anatomy, Meram Medicine Faculty, Necmettin Erbakan University, Konya, Turkey; **Department of Anatomy, Faculty of Medicine, Akdeniz University, Antalya, Turkey

Objectives: In this study, we aimed to conduct morphometric measurements belonging to foramen magnum that forming the craniocervical junction and the major anatomical structures passing inside.

Methods: This study was conducted on 100 human dried skulls (46 male and 54 female) in collections of Necmettin Erbakan and Akdeniz University Anatomy Labratory. Sex determination in human skulls were occured with using topographic points (Superciliary arch, external occipital protuberance). In this study, it was performed that morphometric measurements about foramen magnum (Antero-posterior lenght of foramen magnum-APL1, transverse diamater of foramen magnum (TD), Antero-posterior lenght of occipital condyle (APL2), width of occipital condyle (WOC), the lenght of between the rear end of the occpital condyle and the rear end of the foramen magnum (L1-OC/FM), the lenght of between the front end of the occpital condyle and the front end of the foramen magnum (L2-OC-FM), the distance between right and left condylar fossa). Also, morphological apperance of foramen magnum was classified according to Chethan et al. (2011) as 1Round shape, 2-Egg shape 3-Tetragonal, 4-Oval, 5-Irregular, 6Hexagonal, 7-Pentagonal and 8-Rectangular. Datas were assessed using SPSS 21.0 statistic program.

Results: As a result, it was found that mean APL1 37.00 \pm 2.77 mm (Male: $37.42 \pm 2.44$, Female: $36.65 \pm 3.00$ ), mean TD $32.85 \pm$ 
$5.04 \mathrm{~mm}$ (Male: $33.91 \pm 6.72$, Female $31.94 \pm 2.70$ ). Regarding to APL1 and TD, no statistical differences were found between genders $(p>0.05)$. In addition to this, foramen magnum were found to be tetragonal in the ratio of $32 \%$.

Conclusion: Morphometric measurements of foramen magnum were performed in this study. We believe that the obtained datas will be helpful during surgery of foramen magnum.

Keywords: Foramen magnum, occipital condyle, morphometry

\section{P-29}

\section{Variation of right renal artery}

Keskinoz EN*, Kabakcı Aydın $\mathrm{AD}^{* *}$, Akın D**, Ozbek O***, Ozen $\mathrm{KE}^{* *}$

*Department of Anatomy, Faculty of Medicine, Acıbadem University istanbul, Turkey; **Necmettin Erbakan University Meram Medicine Faculty, Department of Anatomy, Konya, Turkey; ***Department of Radiology, Meram Medicine Faculty, Necmettin Erbakan University Konya, Turkey

Objectives: Renal arteries which supply the kidney originate from anterolateral or lateral branches of abdominal aorta below the superior mesenteric artery at the level of L1-L2 vertebrae. Due to the persistence of embriyonic vessels, lateral branches of mesonephrons, in adulthood, anatomy of the renal artery shows variation. Variation of double renal artery is the most common one among the renal morphological variations.

Case report: A 67 year old male patient who admitted to emergency department with severe abdominopelvic pain.The case showed variation of the renal artery during CT angiography. In the literature, different values ranging from the $11 \%$ to $30.9 \%$ are given related with the variation of root of the renal artery.

Results: According to classical anatomy textbooks in $70 \%$ of individuals represent single renal artery which is derived from the abdominal aorta at the level of $\mathrm{L}_{1}-\mathrm{L}_{2}$ vertebra. However, the description of classical renal vascularization is formed by one artery and one vein is only found less than $25 \%$ of the cases. On the other hand the frequency of variation of the renal artery shows social, ethnical and racial differences.

Conclusion: Failure of transplantation of the kidney is supplied by double renal artery is higher than the kidney is supplied by single artery. We believe that our results in this study will increase the awareness of variations and effect the success of surgical procedure positively.

Keywords: Renal artery, variation, computed tomography

\section{P-30}

Hippocampal volume changes observed in the diabetic rats treated with mianserin

Polat $E^{*}$, Soztutar $\mathrm{E}^{*},{ }^{* *}$, Ucel UI*** Ulupinar $\mathrm{E}^{*},{ }^{* *}$

*Department of Health Science Institute, Interdisciplinary Neuroscience, Eskisehir Osmangazi University, Eskisehir, Turkey; **Department of Anatomy, Faculty of Medicine, Eskisehir Osmangazi University, Eskisehir, Turkey; ${ }^{* *}$ Department of Pharmacology, Faculty of Pharmacy, Anadolu University, Eskisehir, Turkey
Diabetes mellitus is a systemic disease causing complications in many organs such as cardiovascular system, kidney, eye and brain. Changes in the brain are observed as alterations in the expression of neuropeptides, neurotoxicity, astrogliosis and decreased hippocampal synaptic plasticity. Mianserin is a selective 5-HT2A serotonin receptor and partial adrenergic receptor agonist drug mainly used for treatment of the major depression. In this study, we aimed to investigate the effects of Mianserin on the volume of the hippocampus of rats with experimental diabetes mellitus. Rats were divided into 4 groups; healthy controls (HC), diabetes mellitus group received streptozotocin injection $(\mathrm{DM})$ and treatment groups in which Mianserin was administered by oral gavage at the dosage of $30 \mathrm{mg} / \mathrm{kg}(\mathrm{DM}+30 \mathrm{mg} / \mathrm{kg})$ and $45 \mathrm{mg} / \mathrm{kg}(\mathrm{DM}+45$ $\mathrm{mg} / \mathrm{kg}$ ) per day, for two weeks. After completion of the treatment, rats were perfused and sections from their brains obtained by systematic randomize sampling method were stained with the Nissl method. The volumes of dentate gyrus and CA1-3 regions of the hippocampus were estimated by the Cavalieri method. Statistical comparisons of the data were done by employing the one-way variance analysis test. The volume of CA1-3 region in the DM group $\left(1.12 \mathrm{~mm}^{3}\right)$ was significantly $(\mathrm{p}<0.01)$ increased compared to HC group $\left(0.69 \mathrm{~mm}^{3}\right)$. On the other hand, in the Mianserin treated groups, these volumes displayed a reduction in a dose dependent manner $\left(1.01 \mathrm{~mm}^{3}\right.$ and $0.52 \mathrm{~mm}^{3}$ for $30 \mathrm{mg} / \mathrm{kg}$ and $45 \mathrm{mg} / \mathrm{kg}$ doses, respectively). Similarly, volume of the dentate gyrus were significantly higher $(\mathrm{p}<0.05)$ in diabetic rats $(0.59$ $\left.\mathrm{mm}^{3}\right)$ than those of HC group $\left(0.39 \mathrm{~mm}^{3}\right)$. While Mianserin treatment did not cause a significant difference in the volume of the granular and pyramidal cell layer at the dosage of $30 \mathrm{mg} / \mathrm{kg}$, reversed the hippocampal volume to the level of the control groups at the dosage of $45 \mathrm{mg} / \mathrm{kg}$. These differences occurring in the volumes of the hippocampus might originate from the disproportionate volumetric changes in the brain and the hippocampus of the diabetic animals. The positive effects of Mianserin on the hippocampal volume ratios might be explained by the microanatomic cerebrovascular changes, reactive neurogenesis or reduced the level of gliosis.

Keywords: Hippocampus volume, Cavalieri, Mianserin

\section{P-31}

\section{The protective effects of caffeic acid phenethyl ester against testis injury induced by carbon tetrachloride in rats}

\section{Taslidere E, Cetin A, Elbe H, Vardi N, Gul M, Otlu A \\ Department of Histology and Embriyology, Faculty of Medicine, Inonu University, Malatya, Turkey}

Objectives: In the present study, we aimed to investigate the effects of caffeic acid phenethyl ester (CAPE) on testicular injury induced by carbon tetrachloride $\left(\mathrm{CCl}_{4}\right)$

Methods: 28 adult male Wistar-albino rats were divided into 4 groups ( $\mathrm{n}=7)$. Control (5\% ethanol, $1 \mathrm{ml} /$ day/ip), Olive oil ( 0.5 $\mathrm{ml} /$ every otherday/ip), CCl4 (0.5 ml/kg every otherday/ip), $\mathrm{CCl}_{4+} \mathrm{CAPE}(10 \mu \mathrm{mol} / \mathrm{kg} / \mathrm{day} / \mathrm{ip})$. At the end of the study, the 
tissue samples were fixed in $10 \%$ formalin and were embedded in paraffin. Tissue sections were cut at $5 \mathrm{İm}$, mounted on slides, stained with hematoxylin-eosin (H-E). 100 tubules were classified in each slide at a magnification of $20 \mathrm{X}$ as intact, atrophic or degenerated. Sections were examined using a Leica DFC280 light microscope and a Leica Q Win Image Analysis system (Leica Micros Imaging Solutions Ltd., Cambridge, UK).

Results: The testis sections of control and olive oil groups showed normal histological appearance. However in the CCI4 group, $55.00 \pm 4.221 \%$ of tubules were intact, $25.00 \pm 2.67 \%$ of tubules were atrophic, and $20.00 \pm 1.88 \%$ of tubules were degenerative. Also, multinucleated giant cells were observed in some of the seminiferous tubules lumen. In the CCI4 +CAPE group, $72.14 \pm 3.91 \%$ of tubules were intact, $16.42 \pm 2.10 \%$ of tubules were atrophic, and $11.42 \pm 2.36 \%$ of tubules were degenerative. In $\mathrm{CCI}_{4}$ group, number of affected seminiferous tubules were found to be significantly increased when compared with control group $(\mathrm{p}<005)$. On the other hand, the number of affected seminiferous tubules in $\mathrm{CCI}_{4+} \mathrm{CAPE}$ group was statistically significantly lower than in the $\mathrm{CCI}_{4}$ group $(\mathrm{p}<005)$.

Conclusion: We suggest that CAPE may be useful for decreasing the harmfull effects of $\mathrm{CCI}_{4}$.

Keywords: Carbon tetrachloride, CAPE, testis, rat

\section{P-32}

The therapeutic effects of pentoxifylline and caffeic acid phenethyl ester against D-galactosamine induced lung injury in rats

Taslidere E*, Vardi $\mathrm{N}^{*}$, Ates B**, Elbe $\mathrm{H}^{*}$, Y1ldız A*, Karaaslan $\mathrm{M}^{* *}$

*Department of Histology and Embriyology, Faculty of Medicine, Inonu University, Malatya, Turkey; *Faculty of Science and Art, Department of Chemistry, Inonu University, Malatya, Turkey

Objectives: The aim of this study was to investigate histological changes occurred in lung tissue by galactosamine (GAL) and effects of pentoxifylline (PTX) and caffeic acid phenethyl ester (CAPE) on these changes by using histological and biochemical methods in rats.

Methods: In this study, 48 Wistar Albino male rats were used. Rats were divided into 6 groups as follows: control, GAL (500 $\mathrm{mg} / \mathrm{kg})$, GAL+PTX $(20 \mathrm{mg} / \mathrm{kg})$, GAL+ CAPE $(10 \mu \mathrm{mol} / \mathrm{kg})$ PTX and CAPE. Rats, were sacrified and lungs of the rats were removed after 21 days. Samples were processed for light microscopic and biochemical examination. Section were stained with hematoxylin-eosine, and Periodic acid schiff and preparations were examined by using Leica DFC-280 light microscope. Malondialdehyde (MDA) and glutathione (GSH) levels and superoxide dismutase (SOD) and catalase (CAT) activities were assayed.

Results: In histological evaluations, in control groups was seen normal histological structure However in GALgroup were observed hemorrhage, lymphocyte and macrophage accumula- tion. In biochemical analyzes, there was an increase in MDA level, while a decrease was observed in GSH, SOD and CAT activity in group treated to GAL compared to control group. On the other hand, it was seen that, in the group treated to GAL, histological and biochemical injuries in the lung were ameliorated by administration of PTX and CAPE. In these groups, it was found that histopatological score and MDA level were significantly decrease compared to GAL group $(\mathrm{p}<0.05)$ while GSH, SOD and CAT levels were significantly increased $(\mathrm{p}<0.05)$

Conclusion: In this study showed that ameliorative effects of PTX and CAPE on GAL-induced lung injury.

Keywords: Lung, galactosamine, pentoxifylline, CAPE

\section{P-33}

\section{Calculation of morphometric measurements and articular surface area of os coxae}

Atay $\mathrm{E}^{*}, \mathrm{Al} \mathrm{O}^{* *}$, Ertekin T**, Nisari $\mathrm{M}^{* *}$, Unur E**

${ }^{\star}$ First and Emergency Program, Vocational High School of Health Services, Kilis 7 Aralık Universty, Kilis, Turkey; ${ }^{* *}$ Departmant of Anatomy, Faculty of Medicine, Erciyes Universty, Kayseri, Turkey

Objectives: Os coxae, which is involved in the formation of the pelvic skeleton, in terms of its morphometric features are important for sciences such as anatomy, orthopedic and forensic medicine. The aim of the current study was to make a detailed morphometric measurements and to calculate surface area of the articular on dry coxae samples.

Methods: This study was done using a digital caliper sensitive to 0.1 milimeter $(\mathrm{mm})$ on 30 dry coxae samples which were obtained from Department of Anatomy in Erciyes University. The age and gender discrimination wasn't made on dry bone specimens. Morphometric measurements, determined through the 22 parameters, were performed. While 19 of these parameters consist of distance between two points and thicknesses of different parts of the bone, the others were related to determination of articular surface area. Morphometric measurement datas were evaluated by statistical analysis program SPSS16.0.

Results: The mean area of the right and left auricularis surface were calculated as $1614.25 \pm 470.39 \mathrm{~mm}^{2}, 1327.53 \pm 259.79 \mathrm{~mm}^{2}$ respectively. No significant difference was determined between all bilateral morphometric measurements of coxae $(p>0.005)$. To evaluate the correlation between parameters, Pearson correlation analysis was used. Especially, Maximum ilium width (IW) and the shortest distance between Spina iliaca anterior superior (SIAS) and auricularis surface (FA) was observed positively, powerful and meaningful correlation ( $r>0.750$, $\mathrm{p}=0.000)$.

Conclusion: Anatomical features of os coxae are important for many field of sciences such as orthopedic surgical and forensic medicine. Therefore, we hope to available obtained data in these areas.

Keywords: Os coxae, morphometry, articular surface area, anatomy 


\section{P-34}

Immunohistochemical changes in rat testis related to the local beta-hCG injection

Tunc $E^{*}, \underline{\text { Calguner E }} \mathrm{E}^{* *}$, Erdogan $\mathrm{D}^{* * *}$, Elmas $\mathrm{C}^{* * *}$, Oktem $\mathrm{H}^{* * *}$, Goktas $\mathrm{G}^{* * *}$, Gozil R**, Bahcelioglu $\mathrm{M}^{* *}$

*Department of Anatomy, Faculty of Medicine, Maltepe University, istanbul, Turkey; ${ }^{*}$ Department of Anatomy, Faculty of Medicine, Gazi University, Ankara, Turkey; ***Departmert of Histology and Embryology, Faculty of Medicine, Besevler, Gazi University, Ankara, Turkey; $* * *$ Department of Anatomy, Faculty of Medicine, Başkent University, Ankara, Turkey

In recent years, studies made concerning the treatment of obesity, it has been observed that weight loss is accelerated by human chorionic gonadotropin (hCG) hormone preparation used for hypothalamic dysfunction in obesity treatment single dose of subcutaneously to abdominal region. Our aim was to observe immunohistochemical changes in male rats, were fed ad libitum with cafeteria diet to gain weight, related to the local hCG injection in testis. In our study, 10 male obese and 10 non-obese male rats were used. In each group, single dose of subcutaneous beta-hCG injection has been applied to 7 rats for 5 weeks in 5 days of the week, and placebo has been applied to the remaining 3 rats. Following the injection, the tissues were evaluated immunohistochemically. As a result of our studies, there weren't any clear differences in testis and it was thought that the increases and decreases in the number of hormone receptors may be seen in parallel with the increases and decreases in hormone levels. As a conclusion, we believe that the implementation of beta-hCG in addition to diet has no effect on testis.

Keywords: Obesity, beta-hCG, testis

\section{P-35}

\section{Immunohistochemical changes in rat adrenal glands} related to the local beta-hCG injection

Tunc $E^{*}$, Calguner E** Erdogan $\mathrm{D}^{* * *}$, Elmas $\mathrm{C}^{* * *}$, Oktem $\mathrm{H}^{* * * *}$, Goktas $\mathrm{G}^{* * *}$, Gozil $\mathrm{R}^{* *}$, Bahcelioglu $\mathrm{M}^{* *}$

*Department of Anatomy, Faculty of Medicine, Maltepe University, Istanbul, Turkey; **Department of Anatomy, Faculty of Medicine, Gazi University, Ankara, Turkey; ***Departmert of Histology and Embryology, Faculty of Medicine, Gazi University, Ankara, Turkey; $* * * \star$ Department of Anatomy, Faculty of Medicine, Başkent University, Ankara, Turkey

In recent years, studies made concerning the treatment of obesity, it has been observed that weight loss is accelerated by human chorionic gonadotropin (hCG) hormone preparation used for hypothalamic dysfunction in obesity treatment. Our aim was to observe immunohistochemical and ultrastructural changes in rat, were fed ad libitum with cafeteria diet to gain weight, related to the local hCG injection in adrenal glands. In our study, 10 female obese, 10 male obese, 10 non-obese female and 10 non-obese male rats were used. In each group, single dose of subcutaneous beta hCG injection has been applied to 7 rats for 5 weeks in 5 days of the week, and placebo has been applied to the remaining 3 rats. Following the injection, the tissues were evaluated immunohistochemically. As a result of our studies, there weren't any clear differences in adrenal glands and it was thought that the increases and decreases in the number of hormone receptors may be seen in parallel with the increases and decreases in hormone levels. As conclusion, we observed thet the implementation of beta hCG in addition to diet has no effect on adrenal glands.

Keywords: Obesity, beta-hCG, adrenal glands

\section{P-36}

\section{Correlation between postural balance and some foot anthropometric measurements}

Ulucam E, Yilmaz A, Cikmaz S, Parlak M, Donmez D

Department of Anatomy, Faculty of Medicine, Trakya University, Edirne, Turkey

Objectives: Some foot anthropometric data of the human body was to investigate the possible effects on postural balance.

Methods: In our study, after receiving the ethical approval, 17 healthy male subjects were taken. The FDM System Type FDM 1.5 force platform (Zebris ${ }^{\odot}$ Medical GmbH, Germany) was used for measurements. Subjects, foot length, circumference, width and soles circumference was measured with respectively. Then, to be open and closed eyes, static postural balance measurements were made. COP area (center of pressure), PL (path length), APSD (antero-posterior sliding distance), LSD (lateral shift distance) were detected. The relationships between these data were compared statistically.

Results: The correlation between right foot width and COP area (closed eyes) was moderate $(r=-0,29)$. Left foot width showed a moderate correlation with APKM (eyes open) $(r=-$ 0.36). Right foot circumference detected a moderate correlations with the path length $(r=0.28)$ and AKPM ( $r=-0.34)$. APKM with the left foot circumference (eyes open) was in the relationship between moderate $(r=-0.27)$. Other findings were not detected in any relationship.

Conclusion: In our study of the relationship between static postural balance and foot anthropometric measurements were analyzed. Between foot measurements and static postural balance were poor correlation observed. We believe that can be obtained meaningful results by increasing the number of subjects.

Keywords: Static balance, postural, anthropometry, foot.

\section{P-37}

\section{Case of branching variations of coeliac trunk with circumaortik left renal vein}

Turamanlar $\mathrm{O}^{*}$, Kacar E** Horata $\mathrm{E}^{* * *}$, Madan $\mathrm{G}^{* * *}$

*Department of Anatomy, Faculty of Medicine, Afyon Kocatepe University, Afyonkarahisar, Turkey; **Department of Radiology, Faculty of Medicine, Afyon Kocatepe University, Afyonkarahisar, Turkey; ${ }^{* *}$ Atatürk Vocational School of Health Services, Afyon Kocatepe University, Afyonkarahisar, Turkey 
Objectives: Renal veins pass in front of the renal arteries and pour to the inferior vena cava. The left renal vein extends in front of abdominal aorta. Hepatic artery and the splenic artery are terminal branches of the coeliac trunk which arising from the abdominal aorta.

Case report: In a spiral computed tomography of the upper abdomen scans taken from 56 years old female patient; hepatic artery and splenich artery were found to come from different locations on the abdominal aorta and circumaortic left renal vein variations was detected.

Results: Sirkumaortic left renal vein incidence ranges from 0.3 to $6.3 \%$. The location and anatomy of the renal vascular pedicle is of importance for vascular surgeons, general surgeons, traumatologists, urologists, and radiologists during surgical procedures and for diagnostic reasons. In order to attain a correct diagnosis during radiologic procedures and to perform complication-free surgery, knowledge of the anatomy and the congenital anomalies of this area is important. Coeliac trunk branching variation similar to our case is observed $3 \%$. Without full analyze of the anatomical information and vessel variations of the upper abdominal region may lead to a significant risk of error that may lead to fatal complications in the surgical management of this region.

Conclusion: Surgeons should be pay attention to variations of coeliac trunk and circumaortic left renal vein. Knowing of these variations is important to correct diagnosis and to avoid any iatrogenic injury during surgery.

Keywords: Variation, circumaortic left renal vein, coeliac trunk

\section{P-38}

\section{Effects of ozone on nerve regeneration after sciatic nerve cut injury}

Ogut $\mathrm{E}^{*}$, Aslan $\mathrm{M}^{* *}$, Sarıkcıoglu L*, Yıldırım FB*

*Department of Anatomy, Faculty of Medicine, Akdeniz University, Antalya, Turkey; ${ }^{* *}$ Department of Biochemistry, Faculty of Medicine, Akdeniz University, Antalya, Turkey

Objectives: The aim of our study is investigate the effects of ozone on nerve healing in rat peripheral nerve cut model and examined with biochemical methods.

Methods: In our study, 100 adult, male, Rattus norvegicus. Wistar albino rats 400-450 gr were divided into 4 groups. In group control $(n=20)$ no cut injury and no treatment; in group sham-operated $(n=20)$ only operation stress; in group $1(n=30)$ after the dissection of nerve, only cut injury; in group $2(n=30)$ after the dissection of nerve, cut injury and ozone was given 35 $40 \mathrm{ug} / \mathrm{ml}$ and $5 \mathrm{cc}$ with intraperitoneal for 2 months. At the end of the treatment all groups blood samples and dissected sciatic nerves were stored $-80^{\circ} \mathrm{C}$ until the analysis. The homogenates were centrifuged at $4{ }^{\circ} \mathrm{C}, 10000 \mathrm{xG}, 15$ minutes and antioxidant enzymes were analyzed in supernatants. Statistical differences were evaluated with one way variance analysis and tukey test.

Results: Biochemical analysis showed that ozone group induced a significant increase in plasma SOD (superoxyde dis- mutase), CAT (catalase), GPx (glutathione peroxidase) activities $(\mathrm{p}<0.001)$. Statistical differences seen SOD and MDA (malonyl dyaldehite) levels in the ozone group ( $<<0.05)$.

Conclusion: Repetition of ozone administration creates resistance against oxidative stress via inducing antioxidative system. Ozone produced ROS (reactive oxygen species) on plasma in peripheral nerve cut injury model that triggers numerous biochemical way with raising the level of intracellular antioxidant enzymes in plasma and tissue. This can be considered raising the ability of regeneration after nerve cut injury.

Keywords: Intraperitoneal, ozone, ozone therapy

\section{P-39}

\section{Face transplant and sensory innervation}

Boduc E, Ozturk L, Gorur I

Department of Anatomy, Faculty of Medicine, Ege University, Izmir, Turkey

Objectives: The trigeminal nerve gets the sense of the entire face except small area around the angulus mandible and lobulus auricula. Peripheral extensions of the neurons which has the cell body in trigeminal ganglion take a way in the n. ophthalmicus, n. maxillary and n. mandibularis to their functional area. Face transplant operation is expected to do both aesthetic and functional restoration when it comes to repair of defects of faces. However, the sensory improvement mechanism of face transplant has not been fully elucidated.

Methods: In this study, seven male cadavers which were in the Department of Anatomy of Medicine Faculty of Ege University were used.The two of them are seperated for studying face transplantation with ATS (a. temporalis superficialis) and AF (a. facialis) methods as a technic. Received from donor cadavers stage of facial flap has been imitated. The branches of n. trigeminus (n. ophthalmicus, n. mandibularis, n. maxillaris, n. alveolaris inferior which is in the mandible, n. supraorbitalis, n. infraorbitalis and n. mentalis) were disected in the other five cadavers.

Results: For a fully functional sensory recovery of facial tissue and also improving trigeminal nerve damage is still being studied during the postoperative process of the face transplantation. The sense of touch, light touch, sensory discrimination, hot and cold sensory perception was evaluated with the result of tests which were conducted in the first two weeks after the operation, in the fourth month and the sixth month. But that sheds light on cortical sensory perception and proprioceptive feedback mechanism has not been solved yet.

Conclusion: In doing surgery on cadavers that mimicked, despite the rigor of all surgery, during the face transplantation we observed a wide range of tissue destruction is inevitable. We have also determined that this damage is also important for the trigeminal nerve. A mask of the face can not be detected in terms of functionality or social communication can not respond to the expectations of people shows us that the work we do.

Keywords: Face transplantation, sensory innervation of face, n. trigeminus 


\section{P-40}

Investigation of the protective effect of acetylsalicylic acid against central nervous system damage caused by rotenon

$\underline{\text { Kose } E^{*}}$, Ekici $\mathrm{S}^{* *}$, Karademir $\mathrm{Z}^{* *}$, Elbe $\mathrm{H}^{* * *}$, Vardi $\mathrm{N}^{* * *}$, Parlakpinar $\mathrm{H}^{* * * *}$, Sagir $\mathrm{M}^{* * * *}$, Acet $\mathrm{A}^{* * * *}$

*Department of Anatomy, Faculty of Medicine, Inönü University, Malatya, Turkey; **Medical Faculty 6. Class Student, Inönü University, Malatya, Turkey; ${ }^{* *}$ Department of Histology-Embriology, Faculty of Medicine, Inönü University, Malatya, Turkey; ****Department of Pharmacology, Faculty of Medicine, Inönü University, Malatya, Turkey

Objectives: Rotenone has been used an insecticide and piscicide that as a mitochondrial toxin. Recently, it has been used for central nervous system (CNS) damage modelling. In our study, protective effect of the acetylsalicylic acid against CNS damage modelling with rotenone was investigated.

Methods: For this purpose, 28 wistar albino female rats diveded into 4 groups. Group I: Control; Group II (Rotenon): s.c. 3 $\mathrm{mg} / \mathrm{kg}$ rotenone for 7 days; Group III (ASA): p.o. $10 \mathrm{mg} / \mathrm{kg}$ ASA for 7 days; Group IV (ASA+ rotenon): $10 \mathrm{mg} / \mathrm{kg}$ ASA applied before (30-45 minutes) s.c. $3 \mathrm{mg} / \mathrm{kg}$ rotenone injection for 7 days. At the 1st and 8th day of experiment, rotarod, accelerod, hot-plate and tail flick tests performed for evaluated motor coordination, balance and analgesia. Then, all rats were sacrificied by high-dose anesthesia and cerebrum and cerebellum tissues removed for histopathological (H-E) examination.

Results: Histologically, cerebrum and cerebellum tissues have normal appereance in the control and only ASA-treated groups. There were increased perivascular edema, inflammation and neuronal degeneration in the cerebrum specimens of Rotenon-treated rats compared with control. Degenerated Purkinje cell and loss of Purkinje cell were observed in the cerebellum of the rotenon-treated rats. ASA application with rotenone decreased these histopathological damage in the both of cerebrum and cerebellum. Rotenone treatment caused an impairment of the rotard and accelerod tests. ASA application with rotenone improve these tests. In addition, our hot-plate test results were correlated with these findings.

Conclusion: It is thougt that, ASA, which has anti-inflammatory and antioxidant properties, shows beneficial effects on CNS damage due to oxidative stres and inflammation induced by rotenone.

Keywords: Rotenone, CNS damage, acetylsalicylic acid

\section{P-41}

Comparison of ultrasonographic patellar tendon, femoral cartilage thickness and isokinetic muscle strength in football and basketball players

Kafkas ME* $\underline{\text { Kuzllay E }}^{* *}$, Kafkas AS*, Kızılay F*** Durmuş B ${ }^{* * * *}$ *High School, Physical Education and Sports Inönü University, Malatya,

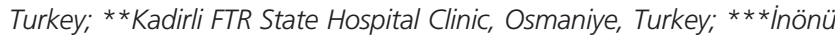
University Turgut Ozal Medical Center Department of Physical Therapy and Rehabilitation, Malatya, Turkey; ${ }^{* * \star}$ Erenköy Physical Therapy and Rehabilitation Training and Research Hospital, Istanbul, Turkey
Objectives: It's aimed that protecting and improving the training plans have been developed to provide guidance that have especially high-risk lower extremity trauma as football and basketball players, taking into account sports injuries incidence, as a result of the dominant side of the knee tendons, cartilage thickness and isokinetic muscle strength measurements.

Methods: Study participated total of 23 athletes form basketball $(n=11)$ and football $(n=12)$ branches. Patellar tendon has width measured along the long transverse axis. Short axis diameter was measured from the midpoint of transverse measurement of this tendon. Femoral cartilage thickness which is including 3-points (midpoint) lateral condyle, medial condyle and the inter-condylar area was measured in the knee fully flexed position. Dominant knee flexion and extension isokinetic muscle strength was measured at $600 / \mathrm{sec}$ angular velocity and hamstring: quadriceps $(\mathrm{H}: \mathrm{Q})$ ratio was measured.

Statistical analysis: Homogeneity tests were done with "Kolmogorov-Smirnov" analysis. The data did not show normal distribution, so "Two Independent Samples T Test" was applied for comparisons between the two branches. All statistical analyzes were performed using SPSS 17.0 software package. Data were expressed as mean $\pm \mathrm{SD}$. The statistical significance level of $\mathrm{p}<.05$ was considered.

Results: Patellar tendon thickness was found $2.16 \pm .43 \mathrm{~mm}$ in basketball, $2.06 \pm .32 \mathrm{~mm}$ in football players. At the dominant knee femoral cartilage thickness (medial-lateral condyle and the inter-condylar region) and patellar tendon thickness measurements no statistically significant difference found between two branches $(\mathrm{p}>0.05)$. In basketball players isokinetic muscle strength $196.40 \pm 8.19 \mathrm{Nm}$ and in football players it was found $210.45 \pm 23.15 \mathrm{Nm}$. Among the branches, dominant knee flexion and extension isokinetic muscle strength which was measured at 600/sec angular velocity and $\mathrm{H}$ : Q ratio were not found statistically significantly different $(\mathrm{p}>0.05)$.

Conclusion: Among athletes of football and basketball branch, the dominant side knee patellar tendon, femoral condylar cartilage thickness and isokinetic muscle strength differences were not statistically significantly different ( $p>0.05)$.

Keywords: Football, basketball, tendon thickness, isokinetic.

\section{P-42}

General anatomical terminology from Nomina Anatomica to Terminologia Anatomica

Geneci $\mathrm{F}^{*}$, Ocak $\mathrm{M}^{*}$, Akdemir Aktas $\mathrm{H}^{*}$, Uzuner $\mathrm{MB}^{*}$, Askit $\mathrm{C}^{* *}$, Sargon $\mathrm{MF}^{*}$

*Department of Anatomy, Medical Faculty, Hacettepe University, Ankara, Turkey; **Ankara Üniversitesi Dil ve Tarih - Coğrafya Fakültesi Latin Dili ve Edebiyatı Anabilim Dalı, Ankara

During the last 120 years, the roadmap of anatomical science, the anatomical terminology, has continuously gone through changes and yet has again rearranged itself. In order to be able to properly and easily understand this terminology made up of thousands of anatomical terms, general anatomical terms are found in the contents of terminological publications. In this 
work, general terminological terms that have been changed and newly added to terminology, found among the 6th edition of Nomina Anatomica (1989) and Terminologia Anatomica (1998) have been studied. It was noticed that 84 new terms found in Terminologia Anatomica were instead missing in Nomina Anatomica. Meanwhile, 2 terms found in Nomina Anatomica, were absent in Terminologia Anatomica. Besides, among these two terminological editions, it was seen that 26 terms had undergone Latin grammatical changes. It was seen that most of the newly added terms, which were mostly composed of the title named 'parts of human body' were added at the beginning of the fragment, and furthermore, these terms were emphasised in related topics. Moreover, it was noticed that the added regional classifications served to make the terminology's content of newly added terms and of some Latin changes more organized and comprehensible. It was also observed that there was not any clear corresponding part of human body related to the found two terms. To sum up, anatomical terminologies are regularly updated and in order to express the terms in the most correct way, studies will still continue in the future.

Keywords: Nomina anatomica, terminologia anatomica, anatomical terminology

\section{P-43}

Alterations on dendritic plasticity of the purkinje neurons in parallel to the environmental conditions

Ergen $\mathrm{FB}^{*}$, Soztutar $\mathrm{E}^{*},{ }^{* *}$, Ay $\mathrm{H}^{* *}$, Ulupinar $\mathrm{E}^{*, * *}$

${ }^{*}$ Department of Health Science Institute, Interdisciplinary Neuroscience, Eskisehir Osmangazi University, Eskisehir, Turkey; **Department of Anatomy, Faculty of Medicine, Eskisehir Osmangazi University, Eskisehir, Turkey

During development, despite the earliest differentiation but the latest completion of its maturation, the cerebellum is one of the most sensitive areas of the nervous system affecting by the adverse environmental conditions. As a consequences of the exposure to positive or negative effects in the perinatal period, alterations occur in the synaptic connections between parallel fibers of the granule cells and dendrites of the Purkinje neurons. In this study, it was aimed to investigate the plastic changes occurring in the Purkinje neurons of the prenatally stressed rats reared in different environmental conditions. Wistar male rats, exposed to immobilization stress in the prenatal period (E14-21), reared in diverse conditions (Enhanced $=\mathrm{EC}$, Standard $=$ SC and isolated = IC) for 6 weeks after weaning. After sacrificing the rats via cardiac perfusion, 150 İm-thick sections from the cerebellar hemispheres were stained with the Golgi-cox method. Analysis of the dendritic spines on the Purkinje cells were done by using the NeuroLucida program. Although there was no differences in the density of spines on the Purkinje neurons of the pups reared in different conditions, significant differences were observed among the percentages of the diverse spine types. Mushroom, thin and stubby spines were distributed equally (34\%, 32\% and $34 \%$, respectively) in the offspring housed in the SC. Whereas in rats housed in the EC, mushroom type spines were increased
(43\%), while the other types were decreased (29\% and 28\%). On the other hand, rats housed in the IC had more thin (37\%) and stubby $(36 \%)$ spines than those of the other groups. As an indication of the more stable type of the synaptic connections, rises in the density of the mushroom spines are in line with the strengthening of the neurotransmission capacity. Therefore, the results obtained in our study indicate that environmental conditions might cause permanent and long-term effects on the plasticity capacity of the animals.

Keywords: Cerebellum, spine, rearing conditions, prenatal stress

\section{P-44 \\ Morphometric evaluation of zygomatic arch in human skull}

Sahin $\mathrm{G}^{*}$, Akın $\mathrm{D}^{*}$, Aydin Kabakci $\mathrm{AD}^{*}$, Sindel $\mathrm{M}^{* *}$, Ay D**, Buyukmumcu MB*

*Department of Anatomy, Meram Medicine Faculty, Necmettin Erbakan University, Konya, Turkey; ** Department of Anatomy, Faculty of Medicine, Akdeniz University, Antalya, Turkey

Objectives: Morphometric evaluation of zygomatic arch in human dried skulls by taking measurements related to bone structue is aimed in this study.

Methods: The study was conducted on human dried skulls among the collection of Necmettin Erbakan and Akdeniz University Labratory of Anatomy. Determination of sex on dried skulls was performed by using various topographic points (Superciliary arch, external occipital protuberance). Dried skulls with one arcus zygomaticus fully intact were also measured beside the ones with both arcus zygomaticus intact. Dried skull with any inconsistency or fructure related to determined measurement points were excluded from the study. Measurements were taken on several points of zygomatic arch (thickness of the anterior, middle and posterior portions of zygomatic arch, distance between anterior and posterior end of the arch, etc.) by single researcher using digital caliper. Data were analyzed using SPSS software version 21.0.

Results: Thickness of arcus among skulls belonging to males, respectively from anterior to posterior have been found $4.84 \pm 1.34 \mathrm{~mm}, 5.04 \pm 1.13 \mathrm{~mm}, 7.2 \pm 1.77 \mathrm{~mm}$ on the right side; $4.85 \pm 1.17 \mathrm{~mm}, 4.9 \pm 1.32 \mathrm{~mm}, 7.58 \pm 2.62 \mathrm{~mm}$ on the left side, where as have been found as; $4.69 \pm 0.86 \mathrm{~mm}, 4.43 \pm 0.95 \mathrm{~mm}$, $7.16 \pm 1.6 \mathrm{~mm}$, on the right side; $4.68 \pm 1.09 \mathrm{~mm}, 4.58 \pm 0.89 \mathrm{~mm}$, $9.82 \pm 2.01 \mathrm{~mm}$ on the left side of the skulls that belong to female. No significant difference has been found between male and female dried skull measurements related to this study.

Conclusion: Measurements taken on zygomatic arch has no significant differance between male and female dried skulls. Data gathered in this study may be usefull for diagnosis and threatment of traumatic events and plastic reconstructive processes related region.

Keywords: Zygomatic arch, morphometry, craniometry 


\section{P-45}

Variations in foramen transversarium of cervical vertebrae: an anatomical study

Yeginoglu $\mathrm{G}^{*}$, Ertemoglu Oksuz $\mathrm{C}^{* *}$, Can MA*

*Faculty of Medicine, Department of Anatomy, Karadeniz Technical University, Trabzon, Turkey; **Vocational School of Health Science, Karadeniz Technical University, Trabzon, Turkey

In this work, anatomical variations of the foramen transversarium of the cervical vertebrae were investigated. Eighty-two foramen transversarium in forty-one cervical vertebrae from our department were studied and classified in four groups: C1, C2, C3-C6, C7. No variation was observed on $\mathrm{C} 1$ and $\mathrm{C} 2$. Variations found on C3-C7 were recorded as: bilateral, unilateral-right, unilateralleft, complete and uncomplete. Results are evaluated and compared with the literature and clinical significance of this variation is discussed by the light of the literature. Anatomical knowledge of these variations in the cervical areas are clinically significant for head-neck surgery and radiology departments as they may affect the course of vertebral vascular bundle.

Keywords: Cervical vertebra, foramen transversarium, anatomical variations

\section{P-46}

\section{The atlas vertebra with double variation}

Yeginoglu G, Can MA

Department of Anatomy, Faculty of Medicine, Karadeniz Technical University, Trabzon, Turkey

Vertebrae forming the vertebral column are classified according to their location and include different features in each region. The vertebrae located in the neck are named "vertebrae cervicales" and two of them located on the top are called Atlas and Axis. They show structural differences from others. Atlas, the first cervical vertebra, doesn't have a body and has two "massa lateralis". The atlas vertebra with a couple variations were detected in the laboratory during the demonstration. One of the variations is there was a abnormal foramen bilaterally behind foramen transversarium, at the junction of the arcus posterior and processus transversus. Other variation is there was a bridge bilaterally on the top of the sulcus arteriae vertebralis on the upper face of arcus posterior. These types of variations are thought to be important for neurologists, neurosurgeons, the radiologist and anthropologists related to clinical cases in this area because the nutritional blood vessels in this area can show differences on account of location and branching.

Keywords: Atlas vertebra, vertebral foramen, variation

\section{P-47}

2.45 $\mathrm{GHz}$ radio frequency radiation exposure induced hippocampal neuron loss and effects of vitamins $\mathrm{E}$ and $\mathrm{C}$

Ozguner $\mathrm{G}^{*}$, Adıgüzel E**, Cömlekci $\mathrm{S}^{* * *}$, Bilkay $\mathrm{C}^{*}$, Dursun $A^{*}$, Ozturk K*, Gungor A***, Sulak O*
*Department of Anatomy, Faculty of Medicine, Süleyman Demirel University, Isparta, Turkey; **Pamukkale University Faculty of Medicine, Department of Anatomy, Denizli, Turkey; ${ }^{* *}$ Department of Telecommunications, Faculty of Engineering, Süleyman Demirel University Isparta, Turkey; $* * * *$ Department of Anatomy, Health Sciences, Pamukkale University, Denizli, Turkey

Objectives: In this study, we aimed to investigate $2.45 \mathrm{GHz}$ radio frequency radiation (EMR) exposure induced changes on rat hippocampal neural numbers and effects of vitamins $\mathrm{E}$ and $\mathrm{C}$. Methods: 28 male Sprague-Dawley rats were used in this study. Rats were divided into three groups: Group I: Control group, (same amount of water given to the group III by oral gavages), Group II: exposed to $2.45 \mathrm{GHz}$ EMR, Group III: exposed to 2.45 $\mathrm{GHz}$ EMR + Vitamins E and C (50 mg vitamin E + $200 \mathrm{mg}$ vitamin C mg / kg body weight / day, oral gavages). Group II and III were exposed to $2.45 \mathrm{GHz} \mathrm{EMR}$, for 60 minutes every day for 30 days. Vitamin E and C, was given 1 hour before the EMR application for Group III. The rats were sacrificed under general anesthesia at the end of 30 days period, and the brains were removed and frozen in a cryostat. Frozen brains were then cut in horizontal plane of $150 \mu \mathrm{m}$ thickness by the cryostat. Sections stained with hematoxylin-esoin (H-E). The number of neurons was estimated using the optical fractional method.

Results: It is observed that the number of hippocampal neurons was reduced in rats exposed to EMR. The hippocampal neuron loss was lower in vitamin $\mathrm{E}$ and $\mathrm{C}$ administered groups when compared to group two. Statistically significant difference was determined between groups.

Conclusion: EMR application induces hippocampal neuron loss in rat brains and vitamin $\mathrm{E}$ and $\mathrm{C}$ have some protective effects.

Keywords: 2.45 radio frequency radiation, hippocampal neuron, rat, vitamin $\mathrm{E}$, vitamin $\mathrm{C}$

\section{P-48}

\section{Vertebral artery arising from the aortic arch}

Sindel $\mathrm{M}^{*}$, Ozgur $\mathrm{O}^{* *}$, Aytaç $\mathrm{G}^{*}$, Sindel $\mathrm{T}^{* *}$

${ }^{*}$ Department of Anatomy, Faculty of Medicine, Akdeniz University, Antalya, Turkey; ${ }^{* *}$ Department of Radiology, Faculty of Medicine, Akdeniz University, Antalya, Turkey

Right and left vertebral arteries originates from the supero-posterior aspect of the subclavian artery in the base of the neck. On the right, subclavian artery arises from the brachiocephalic trunk, on the left it arises from the aortic arch. The left vertebral artery can arise from; the left common carotid artery, the root of the left subclavian artery or directly from the aortic arch. Vertebral arteries derived from a longitidunal canal between the cranial intersegmental arteries in the embryo. If seventh intersegmental artery had fused aortic arch vertebral artery could arise directly from the aortic arch. The prevelance of vertebral artery that derives from aortic arch is reported $1.79-5.8 \%$ in the left, $1-3 \%$ in the right. The aim of this study is to investigate prevelance of the vertebral arteries that originated from aortic arch in the cerebral angiograms retrospectively and indicate the clinical importance of this variation. This study performed in Akdeniz 
University, Faculty of Medicine, Department of Radiology. The origin of left vertebral artery investigated retrospectively in 400 cerebral angiograms that taken for different reasons. In 400 patient 18 (4.5\%) left vertebral arteries that originated from aortic arch are detected. Another vessel variation isn't determined. There are complications associated with anomalous vertebral artery origin as abnormal haemodynamics with intracranial complications, increased likelihood atherosclerosis and dissection risks. Besides awareness of the vertebral artey origin variations is important during the surgical procedures and endovascular applications that related this region.

Keywords: Vertebral artery, serebral angiography, aortic arch

\section{P-49}

Investigation of formation variations of portal vein with multidetector computed tomography

Bolatl $\mathrm{G}^{*}$, Ulusoy $\mathrm{M}^{*}$, Koplay $\mathrm{M}^{* *}$, Acar $\mathrm{M}^{* * *}$, Zararsiz I*

*Department of Anatomy Faculty of Medicine, Mevlana University, Konya, Turkey; ${ }^{* *}$ Department of Radiology, Faculty of Medicine, Selçuk University, Konya, Turkey; ***Department of Physiotherapy and Rehabilitation, Vocational School of Health Services, Mevlana University, Konya, Turkey

Objectives: To determine the types, frequency and clinical implications of formation of variations of portal vein with routine abdominal multi detector CT.

Methods: MDCT images of 182 patients ( 82 male, 100 female) without any pathology were evaluated. Types and frequencies of formation variations of portal vein were determined.

Results: Formation variations of portal vein were observed in $7.6 \%$ in our study population. These variations were observed in 7 male $(8.5 \%)$ and 7 female (7\%) patients. The type of portal vein variation as uniting of superior mesenteric vein, inferior mesenteric vein and splenic vein at the same trunk to form portal vein was determined in 2 males (2.4\%) and 2 females (2\%). Formation of portal vein from inferior mesenteric vein as two branches was observed in 2 male (2.4\%) and 1 female (1\%) patients. Formation of portal vein from left gastric vein uniting with splenic vein was determined in 5 male $(6.1 \%)$ and 4 female (4\%) patients.

Conclusion: Abdominal MDCT imaging was facilitated the detection of formation variations of portal vein. Being aware of these quite common variations would facilitate the determination of probable causes of portal hypertension and will decrease the risk in abdominal surgery.

Keyword: Portal vein, variation, MDCT

\section{P-50}

\section{Cadaver embalming techniques and historical process}

Parlak M, Ulucam E

Department of Anatomy, Faculty of Medicine, Trakya University, Edirne, Turkey

Cadaver is a Latin term derivated from words of "caro data vermibus" (flesh given to worms), means the body of a dead person or corpse. Tahnit (in English embalming) is a term of Arabic origin, which means medication within a certain formula to avoid deterioration of the human or animal body. Embalming cadavers is an issue in anatomy teaching and training that has been wondering by especially medical students and many people. Our study aimed to reveal the recently using embalming techniques and from the past and present development process of the embalming techniques. For this, the literature on the history of embalming, published books on the subject and Turkish Anatomy and Clinical Anatomy of the Association which was published most recently found embalming techniques and methods were investigated. In this study, the results of our; education and training in Anatomy once again been an interesting topic was discussed. This informations will contribute to Anatomy education researchers beginners.

Keywords: Embalming-history, cadaver, anatomy

\section{P-51}

\section{Importance of renal cortical scintigraphy in horseshoe kidney}

Gül SS*, Uysal M**, Koçyiğgit Deveci E***, Irmak Sapmaz $\mathrm{H}^{* * * *}$, Taş $\mathrm{U}^{* *}$

*Department of Nuclear Medicine, Faculty of Medicine, Gaziosmanpasa University, Tokat, Turkey; **Department of Anatomy, Faculty of Medicine, Gaziosmanpasa University, Tokat, Turkey;

$* * *$ Department of Nuclear Medicine, Adana Numune Research and Training Hospital, Adana, Turkey; $* * * *$ Department of Anatomy, Faculty of Medicine, Inonu University, Malatya, Turkey

Horseshoe kidney is fusion anomalies that formed by joining the both kidneys from the midline lower pole. Horseshoe kidney anomaly is accompanied by a high rate of ureteropelvic junction obstruction. Therefore recognition of anomaly is important to avoid the irreversible renal damage even surgical treatment. In this report out of three patients with horseshoe kidneys, it is aimed to introduction and revision of the current literature at the place of renal scintigraphy in the diagnosis and treatment management of this anomaly. Early recognition of horseshoe kidney is valuable for the prevention of kidney damage. In particular, the patients that planned surgery, both the current status of the functional of kidney and abnormality of kidneys can evaluated simultaneously with renal scintigraphic imaging. So scintigraphic imaging has great importance in clinical practice.

Keywords: Horseshoe kidney, fusion anomaly, renal scintigraphy

\section{P-52}

\section{Therapeutic effects of caffeic acid phenethyl ester in streptozotocin-induced diabetic pancreas damage}

Elbe $H^{*}$, Taşlidere E*, Çetin A*, Türköz Y**

*Department of Histology and Embryology, Faculty of Medicine Inonu University, Malatya, Turkey; **Department of Biochemistry, Medical Faculty, Inonu University Malatya, Turkey 
Objectives: In this study, we evaluated histopathological changes and therapeutic effects of Caffeic acid phenethyl ester (CAPE) in streptozotocin (STZ)-induced diabetic pancreas damage.

Methods: 32 male Wistar albino rats were divided into equal 4 groups. Group 1: Control, Group 2: CAPE (10 $\mu$ mole/kg/ip/20 day), Group 3: Diabetes (DM) (55 mg/kg/ip/single dose), Group 4: DM+CAPE. At the end of the study (21 days), pancreas tissues were removed and routin histological procedures were done. Paraffin blocks were cut at $5 \mu \mathrm{m}$ and sections were stained with H-E. Histopathological damage score (acinar cell degeneration, inflammation, islet degeneration, hemorrhage) was calculated $(0-3$, maximum score $=12)$. Sections were examined using a Leica DFC280 light microscope and a Leica Q Win Image Analysis system (Leica Micros Imaging Solutions Ltd., Cambridge, UK). Statistical analysis was carried out using the SPSS 13.0 (SPSS Inc., Chicago, III., USA) statistical program. For comparison Kruskal-Wallis and Mann-Whitney U tests were used. All data are expressed as arithmetic mean \pm SE. $p<0.05$ was regarded as significant.

Results: Control and CAPE groups were normal histologically. In DM group, acinar cell degeneration, inflammation, islet degeneration and hemorrhage were observed. The mean histopathologic damage score $0.25 \pm 0.16$ in group 1 and $0.37 \pm 0.18$. When compared with the DM group $(7.12 \pm 0.47)$ statistically a significant difference was detected $(\mathrm{p}=0.001$, for all). On the other hand, in DM+CAPE group (4.37 \pm 0.32$)$, histopathological changes were reduced $(\mathrm{p}=0.001)$.

Conclusion: We concluded that CAPE has a therapeutic effect via antioxidant property in STZ-induced diabetic pancreas damage.

Keywords: Diabetes, STZ, CAPE, Pancreas damage

\section{P-53}

\section{Therapeutic effects of resveratrol on acethaminophen-induced kidney damage}

Elbe H, Çetin A, Taşlidere E, Otlu A

Department of Histology and Embryology, Faculty of Medicine, Inonu University, Malatya, Turkey

Objectives: Acetaminophen (APAP), an analgesic and antipyretic, is toxic in overdose to the kidney. The aim of present study was to evaluate the renal damage of acethaminophen administration in rats.

Methods: 40 Wistar albino rats were divided into 5 groups. Control, Saline, Resveratrol (RSV) (10 mg/kg/ip), APAP (1000 $\mathrm{mg} / \mathrm{kg} / \mathrm{ip} /$ single dose), APAP+RSV . At the end of the study (24 h) kidneys were removed and routin histological procedures were done. Histopathological damage score (hemorrhage, infiltration, glomerular congestion, vacuolization, loss of microvilli) was calculated $(0-3$, maximum score $=15)$ from H-E and PAS-stained sections. Sections were examined using a Leica DFC280 light microscope and a Leica Q Win Image Analysis system (Leica Micros Imaging Solutions Ltd., Cambridge, UK). Statistical analysis was carried out using the
SPSS 13.0 (SPSS Inc., Chicago, III., USA) statistical program. For comparison Kruskal-Wallis and Mann-Whitney U tests were used. All data are expressed as arithmetic mean \pm SE. $\mathrm{p}<0.05$ was regarded as significant.

Results: Control, saline and resveratrol groups were normal histologically. In APAP group, hemorrhage, infiltration, glomerular congestion, vacuolization and loss of microvilli were observed. The mean histopathological damage scores were $0.37 \pm 0.18$ in group $1,0.12 \pm 0.12$ in group 2 and $0.37 \pm 0.18$ in group 3 . When compared with the APAP group $(8.12 \pm 0.35)$ statistically significant differences were detected $(\mathrm{p}=0.001$, $\mathrm{p}=0.0001, \mathrm{p}=0.001$, respectively). In $\mathrm{APAP}+\mathrm{RSV}$ group (4.75 \pm 0.36$)$, histopathological changes decreased significantly when compared with the APAP group $(\mathrm{p}=0.001)$.

Conclusion: We concluded that resveratrol has a therapeutic effect and reduced histopathological changes on acethaminopheninduced renal damage.

Keywords: Acethaminophen, resveratrol, kidney damage.

\section{P-54}

\section{Investigation of renal artery variations by multidetector CT angiography}

Ulusoy $M^{*}$, Koplay $M^{* *}$, Bolatl $\mathrm{G}^{*}$, Acar $\mathrm{M}^{* * *}$, Zararsı I* ${ }^{*}$ Department of Anatomy Faculty of Medicine, Mevlana University, Konya, Turkey; **Department of Radiology Faculty of Medicine, Selçuk University, Konya, Turkey; ***Department of Physiotherapy and Rehabilitation Vocational School of Health Services, Mevlana University, Konya, Turkey

Objectives: To determine the types, frequency and clinical importance of renal artery variations.

Methods: Renal arteries of 158 patients (77 male, 81 female) who have routine abdominal MDCT angiography imaging were evaluated. Frequency and types of renal artery variations were determined.

Results: Variations of renal artery were observed in 20 male and 20 female patients. Variation at both renal arteries is observed for six of 40 patients (3.8\%). Two types renal artery variations; early arterial branching of renal artery (8.2\%) and extra renal artery $(6.3 \%)$ were observed often in patients. According to entrance to renal artery, early branching of renal artery is classified as hilar renal artery (accessory, 3.2\%) and polar renal artery (aberrant, 3.2\%). Patients with renal artery variations were evaluated clinically. Eleven of patients with renal artery variation have essential hypertension. İt is observed that 9 of these patients have extra renal artery variation and 3 of these patients have early branching variation. 4 extra renal arteries are observed for 5 patients with renal pathology. Nine extra renal arteries and 13 early arterial branching variations are observed for 24 patients without any renal disease.

Conclusion: In this work we determined that renal artery variations are seen often. For diagnosis of essential hypertension, for renal patients and for patients that will have renal transplantation it is important to determine if there are any renal artery variations.

Keyword: Renal artery, variation, MDCT angiography. 


\section{P-55}

Elastofibroma dorsi: radiologic examination of 10 cases

Fazlıogulları $Z^{*}$, Uysal Iİ*, Özbek $\mathrm{S}^{* *}$, Karabulut AK*

*Department of Anatomy, Faculty of Medicine, Selcuk University, Konya, Turkey; **Department of Radiology, Faculty of Medicine, Selcuk University, Konya, Turkey

Elastofibroma dorsi is soft tissue tumor, mostly asymptomatic, beningn and noncapsulated. It is usually located between scapula and thoracic wall. It is firstly identified by Jarvi and Saxen (1961). While the incidence of elastofibroma dorsi is reported as $2 \%$ in asymptomatic, elderly individuals in the assessment of the computerized tomography (CT), it has been showed as $11-24 \%$ in autopsy. It has been reported that it has mostly seen in female individuals of advanced age and especially on the right side. 10 patients, 7 of female and 3 of male, diagnosed as elastofibroma dorsi were assessed according to their thorax CT at Selcuk University Departmant of Radiology. Their mean age was 65 and all of them were asymptomatic except one patient. It is observed that localization of elastofibroma dorsi was bilateral in 6 cases, at the right side in 3 cases and at the left side in 1 case. The average size of $62 \times 26 \times 70 \mathrm{~mm}$ on the right while the left side was measured as $69 \times 28 \times 75 \mathrm{~mm}$. When evaluating its relationship with muscles, it is determined that elastofibroma dorsi mostly localizes deep in serratus anterior muscle at the subscapular region. Meanwhile, elastofibroma dorsi which are in relation to the latissumus dorsi, subscapularis and rhomdoid muscles were also observed. While 9 cases were asymptomatic, only one case, who consulted with symptomatically bilateral mass complaints was surgically resected. Elastofibroma dorsi is easily treated by excision however surgery is not recommended for cases having lesions smaller than $5 \mathrm{~cm}$ and asymptomatic. Since elastofibroma dorsi is a rare lesion and can be easily overlooked in the physical examination, it should be considered in the differential diagnosis and known that it is easily diagnosed by the radiologic methods because of its typical localization and characteristic images of the CT and MR. Thereby it should be taken in to account that incorrect malignancy of diagnosis and following unnecessary biopsy and surgery can be excluded easily.

Keywords: Elastofibroma dorsi, subscapular region, radiology

\section{P-56}

The evaluation of free anatomy applications for smartphones

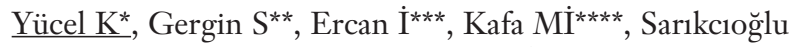
$\mathrm{L}^{* * * * *}$, Öztürk H $\mathrm{H}^{\star * * * * *}$, Tokem Ergür I I******, Kaya $\mathrm{Y}^{\star * * * *}$

*Department of Anatomy, Faculty of Medicine, Yeditepe University Istanbul, Turkey; **Department of Anatomy, Faculty of Medicine, Marmara University Istanbul, Turkey; ${ }^{* * *}$ Department of Biostatistics Faculty of Medicine, Uludag University, Bursa, Turkey; ****Department of Anatomy, Faculty of Medicine, Uludag University Bursa, Turkey; $\star * * * *$ Department of Anatomy, Faculty of Medicine, Akdeniz University Antalya, Turkey; $* * * * * *$ Department of Anatomy, Faculty of Medicine, Mersin University Mersin, Turkey; $* * \star \star * * *$ Department of Anatomy, Faculty of Medicine, Dokuz Eylul University Izmir, Turkey
According to the 2013 data of the Turkish Statistical Institute the rate of individuals using internet by their mobile phones among internet users has almost been doubled compared with that of the previous year $(24 \%-41 \%)$. The use of smartphones is also rapidly increasing among the university students. Many anatomy applications exist for the smartphones. An appopriate anatomy application downloaded on the students' mobile phones who always keep their cell phones with them might ease the process of learning anatomy. For the last few years; smartphone application studies on various medical branches and clinical conditions have taken place in the literature. In these studies, however, either information about the applications was presented or the accuracy of the applications on clinical tests was tested. To our knowledge, there has been no study in the literature so far in which medically relavant smartphone applications have been evaluated quantitatively by the specialists of that medical discipline. Out of the 251 applications at the "Google Store"'related to anatomy 1) free, 2) not a game, 3) not an exam, quiz or "flashcard", 4) not an e-book or on-line lecture, 5) not for kids have been selected for evaluation. There were 29 applications related to more than one system/structure; 6 applications related to one system/structure; and 5 anatomy dictionaries satisfying our criteria. These applications were rated by 13,465 users with an average rating of 4.1 over 5 . It is, however, obvious that the group of these raters is a heterogenous group with an ambiguous background. In our study, the consistency and reliability among the four anatomists and one anatomy resident from five different centers rating the 40 applications would be evaluated. Additionally, two raters would be rating the free anatomy applications at the Apple Store for I-phones. We do believe that it would be beneficial to evaluate the defects of the free anatomy applications for both android and smartphones using IOS operating systems and select the ones those we can recommend to our students.

\section{P-57}

\section{Plantar fasciitis: case report}

Gül SS*, Uysal M**, Hasbek $Z^{* * *}$, Çiçek $M^{* *}$, Taş U**

*Department of Nuclear Medicine, Faculty of Medicine, Gaziosmanpaşa University, Tokat, Turkey; **Department of Anatomy, Faculty of Medicine, Gaziosmanpaşa University, Tokat, Turkey; $* * *$ Department of Nuclear Medicine, Faculty of Medicine, Cumhuriyet University, Sivas, Turkey

The plantar fascia is the thick connective tissue (aponeurosis) which supports the arch on the bottom of the foot. It runs from the tuberosity of the calcaneus forward to the heads of the metatarsal bones. Plantar fasciitis is a common painful disorder affecting the heel and underside of the foot. It is a disorder of the insertion site of ligament on the bone and is characterized by scarring, inflammation, or structural breakdown of the plantar fascia. In this case report, plantar fasciitis with pain in the right foot is presented in a male patient. Three-phase bone scintigraphy with Technetium-99m methylenediphosphonate showed that "Bloody and blood pool phase images confirms the increase of perfusion and hyperemia in the calcaneus and inferior area of the right foot, and the presence of increased focal osteoblastic 
uptake in the inferior part of the right calcaneus was observed in the late-phase static images". The patient was accepted as a case of plantar fasciitis with the help of the scintigraphic, radiographic and clinical findings, and treatment was initiated. The presence of plantar fasciitis should be kept in mind in the differential diagnosis of chronic pain of the foot, and in addition to the radiological method, three-phase bone scintigraphy can also be used in the diagnosis of plantar fasciitis.

Keywords: Plantar fasciitis, plantar fascia, bone scintigraphy

\section{P-58}

Multiple renal artery: a case report

Özen $\mathrm{KE}^{*}$, Öztekin $\mathrm{CH}^{*}$, Akın D*, Özbek O**, Şeker $\mathrm{M}^{*}$

*Department of Anatomy, Meram Medical Faculty, Necmettin Erbakan University, Konya, Turkey; **Department of Radiology, Meram Medical Faculty, Necmettin Erbakan University Konya, Turkey

Objectives: Renal arteries arise from aorta at the level of L1 and L2 vertebrates. Their origins are just below the superior mesenteric artery. Left renal artery's origin is slightly above the origin of right renal artery. Frequency and type variations of renal arteries are reported in either anatomic or arteriographic studies. The most common anomaly is presence of more than one renal artery. Variations of renal arteries are important and may cause serious medical situations like necrosis and ischemia at the corresponding level regardless diameter, route and origin of renal artery.

Case report: Multiple right and left renal arteries are observed in a 55 years old male patient during MDCT examination at Necmettin Erbakan University Meram Medical Faculty Department of Radiology. Diameters of right renal arteries were $0.21 \mathrm{~cm}$ and $0.42 \mathrm{~cm}$ while diameters of left renal arteries were $0.48 \mathrm{~cm}$ and $0.22 \mathrm{~cm}$. Origins were between L1 and L2 vertebrates. Distance to superior mesenteric artery of right renal arteries were $1.85 \mathrm{~cm}$ and $1.91 \mathrm{~cm}$. Distance to superior mesenteric artery of left renal arteries were $1.85 \mathrm{~cm}$ and $2 \mathrm{~cm}$.

Conclusion: Endovascular techniques like balloon angioplasty and stent implantation are common for treatment of renal artery pathologies. Besides these intra-arterial therapeutic techniques, during surgical procedures like renal transplantation or radiologic procedures like renal angiography, variations of renal arteries come into question. We believe that this study has importance for radiologists and surgeons.

Keywords: Renal artery, computerized tomography, variation

\section{P-59}

Development of the female external genital organs during the fetal period

Özgüner G, Öztürk K, Bilkay C, Dursun A, Sulak O, Koyuncu E

Deparment of Anatomy, Faculty of Medicine Süleyman Demirel University, Isparta, Turkey

Objectives: In this study we aimed to determine normal size of the female external genital organs in the human fetus by exam- ining morphological structure and, to form a development scale during the fetal period.

Methods: This study was performed on 113 spontaneously aborted human female fetuses aged between 10 and 40 weeks of gestation. Fetuses without any external and internal pathology or anomaly were included in this study. Fetuses were divided into groups according to gestational weeks, months and, trimester. General external parameters of fetuses were measured. Following parameters belong to the female external genital organs were measured and, types of hymen were determined:

- distance between the posterior - anterior labial commissure

- size of labia majus (length, width and, height)

- bilabial diameter

- size of labia minus (length, width and, height)

- size of clitoris (length, width and, height)

- vertical and horizontal diameters of vaginal orifice,

- distance between the external urethral orifice - vaginal orifice,

- distance between the clitoris and external urethral orifice

- length of perineum

Results: The mean of the each parameter was computed by gestational weeks, months and, trimester groups, and data were presented as mean \pm standard deviation. There were statistically significant differences between trimester groups, except vertical and horizontal diameters of the ostium vagina. The bilabial diameter was measured from midpoint of the lateral labial margin to the midpoint of the opposite lateral labial margin. The bilabial diameter was $1.95 \mathrm{~mm}, 4.36 \mathrm{~mm}, 10.81 \mathrm{~mm}$ and $19.01 \mathrm{~mm}$ in the first, second, third and full term fetuses respectively. We observed four types of hymen; hymen anularis 58 (\%51.3), hymen imperforatus $52(\% 46)$, hymen fimbriatus $1(\% 0.9)$ and, hymen septus 1 (\%0.9). A hymenal tag 1 (\%0.9) was also observed in one fetus. We consider that data obtained from this study, will be useful in the assessment of female external genital organ development in fetal period. Results of the study will also be beneficial for clinicians and further clinic studies.

Keywords: Fetus, female external genital, fetal development, morphometry

\section{P-60}

\section{Avascular necrosis of femoral head tracking of 3-phase bone scintigraphy activity}

Gül SS*, Koçyiğit Deveci E**, Uysal $M^{* *}$, Aytekin $\mathrm{K}^{* * * *}$, Taş $\mathrm{U}^{* * *}$

*Department of Nuclear Medicine, Faculty of Medicine, Gaziosmanpasa University, Tokat, Turkey; ${ }^{* \star}$ Department of Nuclear Medicine, Adana Training and Research Hospital, Adana, Turkey; $* * \star$ Department of Anatomy, Faculty of Medicine, Gaziosmanpasa University, Tokat, Turkey;

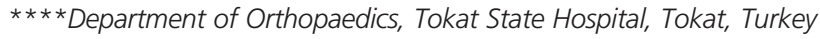

Femoral head avacular necrosis is an aseptic disease that characterized with collapsing and deterioration of nutritional femoral head for any reason. There are classifications according to the radiological examinations. The most frequently used is the Ficat and Arlet staging system. Treatment organized at the patient that come with pain complaint by the $\mathrm{x}$-ray, CT and 
MRI findings of investigations. Although MRI is the gold standard, scintigraphy is more valuable for the diagnostic method in first 5 days by reducing radiopharmaceutical uptake because of hematopoietic cell necrosis at the first 6-12 hours and the osteocytes necrosis at the 12-48 hours. MRI is more sensitive for showing the signal changes in fat cells bone marrow. So; MRI findings can be negative from the beginning of the disease to the fat cell necrosis appear. MRI findings are positive at all stages untill avascular necrosis fully healed. Until fat cell necrosis occurs, contrast-enhanced MRI examination is also reported to be helpful for the diagnosis. In this paper, three-phase bone scintigraphy of avascular necrosis of the right femoral head diagnosed case have presented. At the scintigraphy we observed the osteoblastic activity involvement that center as photopenic and at the arround as increased in femoral head. Contrast-enhanced MRI and three-phase bone scintigraphy is used for the diagnosis of avascular necrosis. Although in the early stages of the disease bone scintigraphy is more sensitive, it can be used for tracking purposes for all stages of the disease. Keywords: Avascular necrosis of the femoral head, whole body bone scintigraphy, technetium 99-m

\section{P-61}

Non-traumatic myositis ossificans: report of three cases Gül SS**, Uysal M**, Koçyiğit Deveci E*** Aytekin K****, Taş $\mathrm{U}^{* *}$

*Department of Nuclear Medicine, Faculty of Medicine, Gaziosmanpasa University, Tokat, Turkey; **Department of Anatomy, Faculty of Medicine, Gaziosmanpasa University, Tokat , Turkey; $* * *$ Department of Nuclear Medicine, Adana Numune Research and Training Hospital, Adana, Turkey; ${ }^{* * *}$ Department of Orthopaedic Tokat State Hospital, Tokat, Turkey

Myositis ossificans (MO), usually was seen after trauma, is an entity that characterized by the formation of new bone in the soft tissue which should not ossifying normally. MO can cause limitation in the joints and pain. Typically occurs in the proximal part of the extremities. Usually seen at 2-3. decades and is more common in men. In this paper, three patients without a history of trauma and atypical located is presented. In the majority of MO cases there is a history of trauma. In MO; fever, edema, sedimentation and high levels of ALP can be seen. The definitive diagnosis is made by X-ray imaging and scintigraphy. Computed tomography and magnetic resonance imaging may be helpful for the diagnosis. X-ray evidence of the disease at the earliest occurs, after 1.5-2 months. In the suspected cases, technetium-99m bone scintigraphy performed with methylenediphosphonate clinch the diagnosis until x-ray findings occur. in the treatment of MO anti-inflammatory drugs, radiotherapy and surgical excision can be used. As a result, it is thought that using the whole body bone scintigraphy for the early and differential diagnosis of MO, showing the extent of the disease is effective.

Keywords: Myositis ossificans, whole body bone scintigraphy, methylenediphosphonate

\section{P-62}

The morfometrical and morphological analysis of the acromion with multidetector computerized tomography

Acar $M^{*}$, Şımşek T*, Ulusoy $M^{* *}$, Zararsız I ${ }^{* *}$, Acar $S^{\star * *}$, Efe $\mathrm{D}^{* * * *}$

*Department of Physical Therapy and Rehabilitation Faculty of Medicine, Mevlana University, Konya, Turkey; ${ }^{* *}$ Department of Anatomy, Faculty of Medicine, Mevlana University, Konya, Turkey; $* * \star$ Department of Physical Therapy and Rehabilitation Konya Numune Hospital, Konya, Turkey; $* * * \star$ Department of Radiology Faculty of Medicine, Mevlana University, Konya, Turkey

Subacromial compression is a clinical condition which appears due to compression of supraspinatus tendon in the subacromial space and causes severe shoulder pain. If subacromial compressions are not treated, this results with rotator cuff ruptures. One of the most important factors for appearance of subacromial compression syndrome and rotaror cuff ruptures is the morphology of acromion. We planned this study as our data would help for differential diagnosis and orthopedics during surgical treatment. This study was conducted on 73 patients (30 females-43 males). For morphometric assessment of the acromion, acromion width (AW), acromion length (AL) and the distance between the acromion and coracoid process (A$\mathrm{CP})$ were measured. We found in 55 (37\%) subjects with flat acromion which is known as Type I, in 71 (48.7\%) subjects with curved acromion which is known Type II and in 20 (13.7\%) subjects with hooked acromion which is known as Type III. To know anatomic details and variations of the region is important for diagnosis and treatment. Furthermore, we think that our study is useful for creation of statistical and reference information of Turkish community.

Keywords: Acromion, variation, MDCT

\section{P-63}

A study on the morphometric development of calcaneal tendon in human fetuses during the fetal period and the evaluation of its clinical importance

Desdicioglu K*, Uguz C ${ }^{* *}$, Sakallı B ${ }^{* * *}$, Koyuncu E ${ }^{* * *}$, Malas MA*

*Department of Anatomy, Faculty of Medicine, Izmir Katip Celebi University, Izmir, Turkey; **Department of Anatomy, Faculty of Medicine, Mugla Sitkı Kocman University, Mugla, Turkey, ***Department of Anatomy, Faculty of Medicine, Suleyman Demirel University, Isparta, Turkey

Objectives: It was aimed that the morphometric development of calcaneal tendon and the structures building it up in human fetuses during the fetal period be anatomically studied and that its clinical importance be evaluated.

Methods: The study comprised a total of 102 fetus legs (51 human fetuses: 26 male and 25 female) whose ages varied between 15-40 gestational week age, without an external pathology or anomaly. The fetuses were divided in groups according to gestational weeks, trimesters and months. In the 
wake of the general external measurements of fetuses, leg dissection was performed. Afterwards, the morphometric parameters of gastrocnemius muscle, soleus muscle and calcaneal tendon were measured.

Results: The averages and the standard deviations of the measured parameters were determined according to gestational weeks, trimesters and months. There was a significant correlation between the measured parameters and the gestational age $(\mathrm{p}<0.001)$. There was no difference between the genders in terms of parameters $(p>0.005)$. All the obtained results were discussed by making a comparison between them and the previous studies made.

Conclusion: We are of the opinion that the data obtained in our study will be of use to the involved clinicians in the evaluation of the development of calcaneal tendon and the structures constituting it during the fetal period, and in clinical studies and applications, as well.

Keywords: Fetal development, morphometry, calcaneal tendon, anatomy.

\section{P-64}

\section{Investigation of lower limb anthropometric measurements at the age of 18-22 students in Cukurova University}

Yücel AH, Özandaç S, Kabakcı AG

Department of Anatomy, Faculty of Medicine, Cukurova University, Adana, Turkey

Objectives: We aimed to investigate lower limb anthropometric measurements, indexes and relationships between measurements at the age of 18-22 students in Cukurova University.

Methods: Lower limb indexes and anthropometric measurements were taken from 90 students (female: 50, male: 40) who are studying in Cukurova University. In our study we have measured demographic data (age, body weight, height, sex and BMI-body mass index-), the length of lower limb, thigh, leg, foot and foot breadth, the circumference measurements of waist, hip, thigh and leg. Measurements, using sliding caliper, digital bascule, stadiometer and non-elastic tape measure were taken. Then, the ratio of lower limb, thigh and leg length to height, the ratio of leg length to lower limb length, the ratio of waist circumference measurement to hip circumference measurement, crural and foot indexes were calculated. SPSS 20.00 were used in this study. Min., max., mean and SD (standard deviation) values were calculated. The study was approved by the Instution's Ethics Commity.

Results: According to the result of measurements performed in students, we got from body weight (female: $60.23 \pm 8.71$, male: $74.3 \pm 9.80 \mathrm{~kg}$ ), height (female: $164.84 \pm 5.39$, male: $176.55 \pm 7.04 \mathrm{~cm}$ ), BMI (female: $22.09 \pm 2.83$, male: $23.84 \pm 2.99$ $\mathrm{kg} / \mathrm{m}^{2}$ ), the ratio of lower limb to height (female: $0.5086 \pm 0.0134$, male: $0.5141 \pm 0.0233$ ), the ratio of thigh lenght to height (female: $0.2126 \pm 0.0197$, male: $0.2267 \pm 0.0227$ ), the ratio of leg length to height (female: $0.1991 \pm 0.0181$, male:
$0.2160 \pm 0.0392)$, the ratio of thigh length to lower limb length (female: $0.4173 \pm 0.0369$, male: $0.4403 \pm 0.0349$ ), the ratio of leg length to lower limb length (female: $0.3919 \pm 0.0338$, male: $0.4228 \pm 0.0927$ ), the ratio of waist circumference measurement to hip circumference measurement (female: $0.7223 \pm 0.0548$, male: $0.8117 \pm 0.0511$ ), crural index (female: $94.01 \pm 8.01$, male: $92.10 \pm 10.91$ ) and foot index (female: $37.19 \pm 3.15$, male: $39.37 \pm 3.23)$.

Conclusion: We compared our findings with those in the literature and assessed them in result of our study.

Keywords: Lower limb, anthropometry, index

\section{P-65 \\ Partial anomalous pulmonary venous return anomaly: case report}

Fazlıŏulları Z*, Karabulut AK${ }^{*}$, Uysal İं* $^{*}$ Nayman A**

${ }^{*}$ Department of Anatomy, Selcuk University, Faculty of Medicine, Konya, Turkey; **Department of Radiology, Faculty of Medicine, Selcuk University, Konya, Turkey

Partial anomalous pulmonary venous return (PAPVR) is a rare abnormality in which one or more pulmonary veins drain into a systemic vein or the right atrium. Anomalous right-sided pulmonary veins might drain into the superior vena cava, inferior vena cava, right atrium, azygos vein, portal vein, or hepatic vein. Besides, anomalous left-sided pulmonary veins could drain into the left brachiocephalic vein, coronary sinus, or hemiazygos vein. Right sided anomalous return is the most common, with left sided anomalous return uncommon. Presented is a case of left upper lobe partial anomalous pulmonary venous connection that was diagnosed incidentally on computed tomography (CT). The patient, 4.5 year-old boy, was admitted to the pediatry clinic of our faculty who was complaining of a persistent cough and a thoracic CT was performed. The lungs were normal, however it was observed that the left superior pulmonary veins were draining into a vertical vein, $24 \mathrm{~mm}$ in length, which then drained into the left brachiocephalic vein. The veins of the right lower and upper lobe were measured as $9 \mathrm{~mm}$ and $8 \mathrm{~mm}$ just before connection of the left atrium respectively and the diameter of the drainage vein of the left lower vein was measured $6 \mathrm{~mm}$. Enlargement in diameter was not observed and the measured value of the diameter of the vertical vein, where it connects to the brachiocephalic vein, is $7 \mathrm{~mm}$. Additionally, no cardiac anomaly was detected in the assessment of the patient's CT and echocardiography. PAPVR usually has no symptoms in the childhood and it is diagnosed in an older age and usually cyanosis is not observed. The decision to perform a surgical correction relies on the number and location of the veins, the magnitude of the left-to-right shunt and associated cardiac anomalies. Knowing these variations has an importance in radiological examinations, catheter placement and surgical procedures.

Keywords: Anomaly, pulmoner vein, computed tomography 


\section{P-66}

A variation of right renal artery duplication with retroaortic left renal vein

Turamanlar $\mathrm{O}^{*}$, Ünlü $\mathrm{E}^{* *}$, Toktaş $\mathrm{M}^{* * *}$, Horata $\mathrm{E}^{* * *}$, Songur $\mathrm{A}^{*}$

*Department of Anatomy, Faculty of Medicine, Afyon Kocatepe Universty, Afyonkarahisar, Turkey; **Department of Radiology, Faculty of Medicine, Afyon Kocatepe Universty, Afyonkarahisar, Turkey; $* * * *$ Department of Anatomy, Faculty of Medicine, Turgut Ozal University, Ankara, Turkey; ${ }^{* *}$ Department of Orthopedic Prosthesis - Orthosis, Atatürk Vocational School Of Health Services, Afyon Kocatepe Universty, Afyonkarahisar, Turkey

Accessory renal artery is frequently encountered variation which occurs in $20-30 \%$ of the population 5 . It may not be clinically significant when it is without any accompanying variation. However, when it occurs with other variations, the incidence in population is lower and there is a greater probability that the variations will result in pathological conditions. Renovascular variations are of particular importance in the treatment of renal and renovascular conditions. Multiphasic upper abdominal computed tomography of a 68 year old male patient with renal cell carcinoma of right kidney revealed duplication of right renal artery and retroaortic left renal vein variation. Differential diagnosis should be made with great care in patients with renal neoplasms since variations may complicate diagnostic procedures. Here we present a rare variation. Knowledge of variations accompanied by pathologies as in this case may be important for the diagnosis and treatment.

Keywords: Variation, retroaortic left renal vein, duplicated right renal artery

\section{P-67}

Comparative evaluation of static and dynamic balance between basketball players and sedentary group

Uzun $A^{*}$, Kabadayı $M^{* *}$, Özdemir F*, Gölpınar $M^{*}$, Altunsoy $E^{*}$, Nahir $M^{*}$, Uysal $A^{* *}$

*Department of Anatomy, Faculty of Medicine, Ondokuz Mayıs University, Samsun, Turkey; **Faculty of Yaşar Doğu Sport Sciences, Ondokuz Mayıs University, Samsun, Turkey

The aim of the present study is to describe and compare between basketball players and sedentary group in the dynamic balance, static balance as open and closed eyes performance. The study included 20 students from Faculty of Yaşar Doğu Sports Sciences players of the basketball team and 19 volunteer sedentary students of Ondokuz Mayis University. Taking measurements of the isokinetic balance measurement instrument (CSM brand Prok tecnobody) was used. The dynamic balance test was applied in double-leg stance position. Static balance was measured in double-leg stance position as open and closed eyes, respectively. The data was analyzed using independent sample t-test; $p$ value at 0.05 or less was accepted as significant difference. The average track error in dynamic balance test of basketball players and sedentary group were $30 \pm 15.67 \%$ and $20.78 \pm 11.41 \%$, respectively. The static balance test scores in static balance as open eyes of basket- ball players and sedentary group were $14.55 \pm 8.84,11.50 \pm 3.84$ and as closed eyes were $11.50 \pm 3.84$ and $9.89 \pm 2.68$, respectively. The dynamic balance of the sedentary group performance were lesser average track error than basketball players $(\mathrm{p}<0.05)$. There were no statistically significant differences in static balance performance between basketball players and sedentary group ( $>0.05$ ). Dynamic balance performance in basketball players was lower than sedentary group, due to their physical properties.

Keywords: Dynamic balance, static balance, basketball player, sedentary group

\section{P-68}

\section{The effect of the diameter of the lower calyx} infundibulum on the success of ESWL

\section{Köse E*, Oğuz F** Beytur A**}

*Department of Anatomy, Faculty of Medicine, Inonu University, Malatya, Turkey; **Department of Urology, Faculty of Medicine, Inonu University, Malatya, Turkey

The success of the treatment of lower calyx stones less than other calices because of the structure of lower pole kidney. The infundibulum, anatomical section between calicies and renal pelvis, is one of the factors that affect the broken stones thrown out of the calyx. Extracorporeal shock wave lithotripsy (ESWL) is widely using for treatment kidney stones extensively. In this study, the effect of the diameter of the infundibulum on the success of ESWL was investigated by analysis the data from patients with lower pole calyx stones. The patients divided into two groups; group I: the diameter of infundibulum $4^{5}$ $\mathrm{mm}$, group II: the diameter of infundibulum $>5 \mathrm{~mm}$. In conclusion, when considering the patients have similar stone characteristics, the success of the lithotripsy in patients with infundibular diameter large than $5 \mathrm{~mm}$ more than others.

This study published in İnönü Üniversitesi Sağlık Bilimleri Dergisi 2013;1:43-45.

Keywords: Kidney stone, lithotripsy, infundibulum

\section{P-69}

\section{Morphometry of groove of vertebral artery}

Örüncü MB*, Özer $M^{*}$, Örten $S^{*}$, Özdemir T*, Çakır T*, Ateş $\mathrm{C}^{* *}$, Esmer $\mathrm{AF}^{* * *}$

*Ankara Üniversitesi Tıp Fakültesi Dönem IV Öğrencileri, Ankara, Turkey; ${ }^{*}$ Ankara Üniversitesi Tıp Fakültesi Biyoistatistik Anabilim Dalı, Ankara, Turkey; ***Department of Anatomy, Faculty of Medicine, Ankara University, Ankara, Turkey

The vertebral artery can be damaged during the surgery of foramen magnum or posterior cranial fossa and this damage can cause serious neurologic deficits, loss of consciousness and cardiovascular or respiratory problems. The goal of our study is to explore the groove of vertebral artery to reduce the risk of the damage of vertebral artery. 86 atlas were studied in this study. The development of canal for vertebral artery, the distance between posterior tubercle and medial and lateral margin 
of groove of vertebral arrtery were recorded. The mean distance between posterior tubercle and medial margin of groove was $12.2 \pm 2.4 \mathrm{~mm}$ on left side and $11.6 \pm 2.6 \mathrm{~mm}$ on right side. The mean distance between posterior tubercle and lateral margin of groove was $16.2 \pm 2.9 \mathrm{~mm}$ and $15.4 \pm 3.2 \mathrm{~mm}$ on left and right side respectively. We determined the distance of midline of vertebral artery and detect the surgical safe zone for this region. And also recorded the development of the canal for vetebral artery which can be related to entrapment syndromes.

\section{P-70}

\section{Morphometry and topographic anatomy of supraorbital notch}

Aşık E*, Kılınç E*, Akan F*, Ertekin T*, Solmaz ZS*, Oztuna $\mathrm{D}^{* *}$, Esmer $\mathrm{AF}^{* * *}$

*Ankara Üniversitesi Tıp Fakültesi Dönem IV Öğrencileri; Ankara, Turkey; ${ }^{* *}$ Ankara Üniversitesi Tıp Fakültesi Biyoistatistik Anabilim Dalı, Ankara, Turkey; ***Department of Anatomy, Faculty of Medicine, Ankara University, Ankara, Turkey

Objectives: After leaving supraorbital notch or foramen, the supraorbital nerve which is one of the terminal branch of frontal nerve innervates the area between upper eyelid and lamboid suture. For the blockage of this nerve, the supraorbital notch where the nerve is unbranched is the convenient point for using less anesthetic and reducing the number of injection. The purpose of this study is to evaluate the morphometry of this notch and determine the correct point of injection.

Metods: The distance between the notch and midline and the height of the notch according the nasion were measured on 50 adult craniums by using digital caliper.

Results: The mean distance between the notch and midline was $21.4 \pm 3.86 \mathrm{~mm}$ on left side and $21.6 \pm 4.76 \mathrm{~mm}$ on right side. The mean height of the notch according the nasion was $9.2 \pm 3.05 \mathrm{~mm}$ bilaterally. Thedevelopment of the supraorbital foramen was found in $30 \%$ of right orbits and $26 \%$ of left orbits. And it was obvious that the entrapment neuropathy of this nerve is more likely in supraorbital foramen than notch.

\section{P-71}

\section{Mastoid process: a craniometric investigation in human skulls}

Aydın Kabakçı AD*, Akın D*, Büyükmumcu $M^{*}$, Sindel $M^{* *}$, Öğ̈ut E**, Yilmaz MT*, Şahin $\mathrm{G}^{*}$

*Department of Anatomy, Meram Medicine Faculty, Necmettin Erbakan University, Konya, Turkey; **Department of Anatomy, Faculty of Medicine, Akdeniz University, Antalya, Turkey

Objectives: In this study, we aimed to conduct morphometric measurements belonging to mastoid process in human dried skulls.

Methods: This study was conducted on 93 human dried skulls in collections of Necmettin Erbakan and Akdeniz University
Anatomy Labratory. Sex determination in human skulls were occured with using topographic points (Superciliary arch, external occipital protuberance). In this study, it was performed that morphometric measurements about mastoid process (Medio-lateral diamater of mastoid process, antero-posterior diamater of mastoid process, the distance between asterion and the root of the zygomatic arch, the distance between asterion and apex of the mastoid process, the distance between asterion and suprameatal spine, the distance between asterion and porion, the distance between proion and apex of the mastoid process, the distance between opisthion and apex of the mastoid process, the distance between, bimastoid breadth, area of the mastoid triangle). Also, the localization of asterion was classified according to franfurkt horizontal plane as 0-same level, 1-above, 2-below. Data were assessed using SPSS 21.0 statistic program.

Results: As a result, it was found that mean lenght of right mastoid process $26.22 \pm 5.46 \mathrm{~mm}$ and left mastoid process $26.93 \pm 3.71 \mathrm{~mm}$ in males; mean lenght of right mastoid process $24.94 \pm 5.49 \mathrm{~mm}$ and left mastoid process $24.95 \pm 4.64 \mathrm{~mm}$ in females. The mean mastoid breadth was determined 106.07士 $4.44 \mathrm{~mm}$ in males, $101.26 \pm 7.30 \mathrm{~mm}$ in females. A significant difference was found between genders in lenght of left mastoid process, the distance between right asterion and porion, the distance between opisthion and apex of the mastoid process, area of right mastoid triangle $(\mathrm{p}<0.05)$.

Conclusion: Morphometric measurements of mastoid process were performed in this study. We believe that the obtained data will be helpful during posterior and posterolateral surgery of skull.

Keywords: Mastoid process, morphometry, craniometry

\section{P-72}

\section{An osteometric study on humerus bones}

Aydın Kabakçı AD, Akın D, Büyükmumcu M, Yilmaz MT, Çiçekcibaşi AE, Cihan E

Department of Anatomy, Meram Medicine Faculty, Necmettin Erbakan University, Konya

Objectives: Humerus is the longest and thickest bone of the upper extremity. As a long bone, it has two ends including bone body, upper ends and lower ends. In this study, we aimed to conduct morphometric measurements belonging to human humerus bones.

Methods: This study was conducted on 60 femur (28 right, 32 left) in collections of Necmettin Erbakan University Meram Medicine Faculty Anatomy Labratory. Digital calipers, osteometric board and precision scales were used for humerus bone measurements. Measurements of humerus bone were classified as measurements of upper end, measurements of bone body and measurements of lower end. Each bone weight was determined with the help of precision scales. Also nutrician foramen number and localization was determined.

Results: In this study, it was determined that mean right humerus lenght $30.41 \pm 1.73 \mathrm{~mm}$, mean left humerus lenght 
$30.04 \pm 2.39 \mathrm{~mm}$. It was identified that mean right humerus weight was $115.05 \pm 28.06 \mathrm{gr}$, mean left humerus weight was $111.63 \pm 33.34$ gr. In $9(15 \%)$ humerus, supratrochlear foramen has been observed. 6 of them were oval and 3 of them were round. Nutrician foramen has been observed in 2 (3.3\%) humerus. Also, it was identified medium and weak correlation between many parameters.

Conclusion: Morphometric features of humerus were performed in this study particularly.We believe that the obtained datas from this study may be qualities of reference for sex determination from humerus.

Keywords: Humerus, morphometry, osteometry

\section{P-73}

Drugs used in pregnancy effects on fetal development

Nalbant A, Yüzbaşığlu N, Şakul BU

Department of Anatomy, Faculty of Medicine, Istanbul Medipol University, İtanbul, Turkey

Although drug use during pregnancy to restrict, in some situations the mother have to using drug. However, the use of these drugs can adversely affect fetal development. This study is a short review, in order to shed light on new studies of drugs used during pregnancy to evaluate the effects on fetal development. When used antiepileptic drugs during pregnancy as diphenylhydantoin are causes, a decrease in fetal and placental weight, fetal morphological changes, delayed ossification, visceral and skeletal variations in the structures. Again some antiepileptic drugs was observed to increase abnormal fetal movement skills engine, autistic features and congenital malformations. The use of corticosteroids in pregnancy have been found to be increased risk of premature labor, with fetal growth and deleterious effects on brain functions. Particularly betamethasone was reduce to cerebral myelination depending on the stage of brain development. Pain reliever and fever reducer capable of acetaminophen use during pregnancy caused an abortion, stillbirth or premature birth and fetal malformations. Magnesium sulfate is used to preeclampsia and eclampsia in pregnancy and causes mean blood flow velocity in the fetus and can reduce the time and cerebral palsy, premature or stillbirth. Using psychoactive drugs for maternal depression, according to their content, causes the fetus to the nutrients and oxygen to a decrease, brain development affects fetal hypoxia, spontaneous abortion increases the risk of low birth weight. In addition, thyroid drugs used in pregnancy affects the development of the fetus, neurophysiological, antihistamines have also been identified to be cause the intrauterine growth retardation and fetal death. When used drug during pregnancy fetal development is largely a negative impact. Therefore, a good way to understand the pathophysiology of fetal and maternal pregnancy in order to assess further studies are needed with pharmacotherapy.

Keywords: Drug use during pregnancy, fetal development, antiepileptics, corticosteroids, diabetic drugs

\section{P-74}

The protective effects of nerolidol against thioacetamide-induced induced liver damage in Wistar albino rats

\author{
Cetin $\mathrm{A}^{*}$, Çiftçi $\mathrm{O}^{* *}$, Başak $\mathrm{N}^{* * *}$
}

*Department of Histology and Embryology, Faculty of Medicine, Inonu University, Malatya, Turkey; ${ }^{*}$ Department of Medical Pharmacology, Faculty of Medicine, Inonu University, Malatya, Turkey; ***Department of Pharmaceutic Toxicology, Faculty of Pharmacy, Inonu University, Malatya, Turkey

Objectives: Thioacetamide (TAA) is a well-known toxicant and its long term exposure could induce liver fibrosis and cirrhosis. Nerolidol (3,7,11-trimethyl-1,6,10-dodecatrien-3-ol) is a sesquiterpene alcohol found in essential oils from plants such as Baccharis dracunculifolia. This study was planned to investigate protective effects of nerolidol to histopathological changes in TAA induced liver tissue.

Methods: In this study, twenty eight male Wistar Albino rats randomly divided into 4 groups of 7 as follows: Group 1: Control, Group 2: TAA (200 mg/kg/ip), Group 3: Nerolidol (100 mg/kg/ gavage); Group 4:TAA (200 mg/kg/ ip) + Nerolidol (100 mg/kg/gavage). Rats were sacrified under anesthesia and liver tissue samples were removed for histological and biochemical investigation. Tissues processed by routine tissue techniques and embedded in paraffin. 5- $\mu \mathrm{m}$ thick sections of tissues were cut, mounted on slides, stained with Hematoxylin-Eosin (H-E) and exam-ined under Leica DFC280 light microscope, Leica Q Win and Image Analysis System. We determined TBARS, SOD, CAT, GPx and GSH levels as spectrophotometric methods.

Results: The results indicated that nerolidol prevents oxidative damage caused by TAA via decrease lipid peroxidations (TBARS) and increase antioxidant defense systems (SOD, CAT, GSH, GPx). With histopatological evaluation, in TAA group such as radial arrangement of hepatocytes were disrupted with TAA exposure. Moreover, eosinophilic stained and pyknotic nuclei cells, mononuclear cell infiltration, vascular congestion and cellular swelling were observed in TAA group. On the other hand, in TAA+Nerolidol group, histopathological findings were not as extensive as in the TAA group.

Conclusion: In conclusion, it was determined that TAA caused the adverse effects as histological alterations and oxidative stress in liver tissue of rats. However, nerolidol treatment eliminated these toxic effects.

Keywords: TAA, nerolidol, hepatotoxicity, oxidative stress

\section{P-75}

Anthropometric analysis of orbital region and age-related changes in adult women

Koç $F^{*}$, Deniz $M^{*}$, Uslu Aİ*, Doğru $S^{* *}$

*Department of Anatomy, Faculty of Medicine, Harran University, Sanliurfa, Turkey; **Vocational School of Health Services, Harran University, Sanlıurfa, Turkey 
Objectives: Normal morphometric values of the orbital region; vary according to age, sex and ethnicity. The purpose of this study was to determine the normal values of orbital region and analysis age related changes in adult women.

Methods: Measurements were performed on 311 healthy women, aged between 20 and $87(47.80 \pm 15.05)$. Subjects were divided into three age groups: young (20-44), middle aged (45-59) and old adults (60 and up). The anthropometric measurements of orbital region were performed on the photographs by using IT version 3.00 software programs.

Results: The mean values of interpupillar distance and nasofrontal age were $64.20 \pm 5.06 \mathrm{~mm}, 149.21 \pm 9.12^{\circ}$, respectively. In young, middle and old groups the mean values of outer canthal distance were $92.98 \pm 5.85 \mathrm{~mm}, 91.00 \pm 7.04 \mathrm{~mm}$ and $88.02 \pm 6.13 \mathrm{~mm}$; inner canthal distance were $31.30 \pm 3.07 \mathrm{~mm}$, $31.72 \pm 3.51 \mathrm{~mm}$ and $31.66 \pm 3.44 \mathrm{~mm}$; palpebral fissure area were $163.41 \pm 29.98 \mathrm{~mm}^{2}, 144.01 \pm 30.90 \mathrm{~mm}^{2}$ and $129.11 \pm 27.21$ $\mathrm{mm}^{2}$; palpebral fissure length were $29.96 \pm 1.76 \mathrm{~mm}, 28.45 \pm 1.96$ $\mathrm{mm}$ and $26.81 \pm 1.95 \mathrm{~mm}$; palpebral fissure width were $9.27 \pm 1.21 \mathrm{~mm}, 8.57 \pm 1.25 \mathrm{~mm}$ and $7.98 \pm 1.18 \mathrm{~mm}$; inner canthal angle were $39.29 \pm 6.04^{\circ}, 37.57 \pm 6.58^{\circ}$ and $35.78 \pm 5.80^{\circ}$; outer canthal angle were $53.90 \pm 8.90^{\circ}, 51.48 \pm 8.87^{\circ}$ and $49.87 \pm$ $9.78^{\circ}$; visible iris area were $96.04 \pm 15.04 \mathrm{~mm}^{2}, 88.63 \pm 16.27 \mathrm{~mm}^{2}$ and $81.73 \pm 14.03 \mathrm{~mm}^{2}$ respectively. There was no significant difference in the interpupillary distance and inner canthal distance between ages $(\mathrm{p}>0.05)$. There was a significant difference in the other measurements between in three age groups $(\mathrm{p}<0.05)$.

Conclusion: We believe that knowing of the normal size of the structures in the orbital region and age-related changes; will be useful to maintain the facial proportions in the plastic and reconstructive surgery and to protect aesthetic appearance of the face.

Keywords: Anthropometry, orbital region, face, women, age

\section{P-76}

\section{Nuclei that are newly added to the brainstem and divided to lower groups}

Akdemir Aktas $\mathrm{H}^{*}$, $\underline{\text { Uzuner } \mathrm{MB}^{*}}$, Geneci $\mathrm{F}^{*}$, Ocak $\mathrm{M}^{*}$, Askit $\mathrm{C}^{* *}$, Sargon $\mathrm{MF}^{*}$

*Department of Anatomy, Faculty of Medicine, Hacettepe University Ankara, Turkey; **Department of Ancient Languages and Literatures, Faculty of Languages, History and Geography, Ankara University, Ankara, Turkey

Central nervous system has always maintained its secrecy even though there have been new developments in the science of anatomy during the years. Many new terms related to the central nervous system have been added to Terminologia Anatomica which was published in 1998. A large part of these terms is composed of brainstem's nuclei and tracts. In this paper, brainstem's nuclei that are found in Nomina Anatomica (1989) and in Terminologia Anatomica (1998) have been compared. In Terminologia Anatomica, 29 new nuclei were noticed to have been added to medulla oblongata, 8 nuclei were added to the pons and 21 new nuclei were added to the mesencephalon. Moreover, it is seen that nuclei that are mentioned in Nomina Anatomica and in Terminologia Anatomica are further divided into lower groups. It was noticed that the seven nuclei in medulla oblangata, the eight nuclei in the pons and the three nuclei in the mesencephalon were divided into lower groups. To sum up, the existence of a high number of the nuclei added to the brainstem and divided to lower groups reveals the fact that the nuclei are recently being discovered as a result of clinical and scientific studies of central nervous system and at the same time it shows the high possibility for them to become a source of new studies in the future.

Keywords: Brainstem, nucleus, terminologia anatomica

\section{P-77}

\section{Anatomical terminology of cranial nerves: changes and additions made in the last 25 years}

Uzuner $\mathrm{MB}^{*}$, Geneci $\mathrm{F}^{*}$, Ocak $\mathrm{M}^{*}$, Akdemir Aktas $\mathrm{H}^{*}$, Askit $\mathrm{C}^{* *}$, Sargon $\mathrm{MF}^{*}$

*Department of Anatomy, Faculty of Medicine, Hacettepe University Ankara, Turkey; ${ }^{*}$ Department of Ancient Languages and Literatures, Faculty of Languages, History and Geography, Ankara University, Ankara, Turkey

Language is a living asset; it gets developed simultaneously together with the development of humanity. $\left(^{*}\right)$ Wilhelm von Humboldt defines language as follows: "Language is not itself a product, it's an activity". Every language forms the spirit of a nation and on the other hand the nation's spirit reveals that language. In this same way, Latin language constitutes the spirit of anatomy and at the same time this language is manifested in anatomy's spirit. In this paper, we discuss the comparison of the terms related to cranial nerves found in Nomina Anatomica (published in 1989) and Terminologia Anatomicalarda (published in 1998), based on their terminology. Changes were noticed to have happened in 18 Latin terms. We could see that 15 of these Latin terms had undergone grammatical changes, 2 of them had undergone a change in meaning and 1 of them had gone through both a grammatical change and a change in meaning. If an assessment of Latin anatomical terms regarding grammar is made, it can be seen that some nouns have been transformed into adjectives, while other ones have been converted from singular to plural form. It was noticed that the terms that were analysed based on their meaning were brought into a more specific state and because of this fact, the localizations of the defined terms were understood more easily.In the examination of the roots of the anatomical terms ; it was observed that a very few terms were included to terminology from Greek language. To conclude; in order to find the most suitable and correct terms, updates in anatomical terminology today still continue and they are likely to go on also in the future.

${ }^{*}$ ) Bedia Akarsu, "Wilhelm von Humboldt'ta Dil-Kültür BağlantıS1, İst 1955, Dil ve Kültür başlıkları

Keywords: Cranial nerves, Wilhelm von Humboldt, nomina anatomica 


\section{P-78}

Examination by elliptical formula and Cavalieri principle of sinus maxillaris in the newborns

Degermenci $\mathrm{M}^{*}$, Ertekin $\mathrm{T}^{*}$, Ulger $\mathrm{H}^{*}$, Acer $\mathrm{N}^{*}$, Coskun $\mathrm{A}^{* *}$

*Department of Anatomy, Faculty of Medicine, Erciyes University, Kayseri, Turkey; **Department of Radiology, Faculty of Medicine, Erciyes University, Kayseri, Turkey

The growth of paranasal sinuses starts prenatal and they continue growing along the all directions together with nasal cavity and other structures on face between the ages of 1 and 7 . As well as the sizes of paranasal sinuses varies between individuals, it may also exhibit different levels of growth in both sides of an individual. It is hard to determine the difference between normal and pathalogical sizes of paranasal sinuses of children and the newborn. In this study, it is aimed to identfy the volume of sinus maxillaris of the newborns by using two different methods and to determine relation between these two methods. The volume of maxillary sinuses were calculated on CT images of 20 individuals, (including 10 males and 10 females) between ages 0 and 1, having been scanned retrospectively in PACS which is used from Erciyes University. The average volume of right sinus maxillaris of male and female individuals were calculated by using eliptical formula as $0.49 \pm 0.34 \mathrm{~cm}^{3}$ and $1.41 \pm 2.53 \mathrm{~cm} 3$ respectively while the average volume of left sinus maxillaris of male and female individuals were measured as $0.52 \pm 0.42 \mathrm{~cm} 3$ ve $1.44 \pm 2.31 \mathrm{~cm}^{3}$. On the other hands, the stereological methods showed that the average volume of right sinus maxillaris of male and female individuals were measured as $0.55 \pm 0.39 \mathrm{~cm}^{3}$ and $0.54 \pm 0.42 \mathrm{~cm}^{3}$ respectively while the average volumes of left sinus maxillaris of male and female were $1.34 \pm 2.22 \mathrm{~cm}^{3}$ and $1.29 \pm 1.99 \mathrm{~cm}^{3}$. No statistically significant difference between male and female individuals were determined according to the measurements $(\mathrm{p}>0.05)$. When two methods are compared, statistically significant difference between two methods was determined only for the volume measurements of right sinus maxillaris of female individuals $(\mathrm{p}<0.05)$. We think that our study will be helpful for the other future studies about examination of volume of sinus maxillaris of the newbors preoperatively and intraoperatively.

Keywords: Stereology, elliptical, maxillary sinus, cavalieri principle

\section{P-79}

\section{Femoroacetabular impingement syndrome:} magnetic resonance imaging findings

Durur Subası I*, Sunar $M^{* *}$, Durur Karakaya A***, Kapakin $\mathrm{S}^{* * * *}$

*Department of Radiology, Faculty of Medicine, Atatürk University, Erzurum, Turkey; **Department of Anatomy, Faculty of Medicine, Erzincan University, Erzincan, Turkey; ${ }^{* *}$ Department of Radiology, Regional Training and Research Hospital, Erzurum, Turkey; $* * * *$ Department of Anatomy, Faculty of Medicine, Ataturk University, Erzurum, Turkey

Objectives: Magnetic resonance imaging findings of femoroacetabular impingement syndrome is aimed to present.
Case report: Magnetic resonance imaging findings of the patients with cam and pincer type femoroacetabular impingement syndromes are presented. Cam type femoroacetabular impingement syndrome shows a bump-like structure at the head-neck region of femur. Pincer type femoroacetabular impingement is observed with overcoverage in the acetabulum.

Conclusion: In recent years, for non-dysplastic hip joints, the concept of femoroacetabular impingement syndrome has began to be considered as the cause of early-onset osteoarthritis. Femoroacetabular impingement is basically thought to be result from repettative shear stresses between the femoral head and acetabular ring during large range of motion. Although lack of clarity this may be due to femoral or acetabular factors. Basically two forms of femoroacetabular impingement have been described: Cam and pincer. Forms between these two are also available. Cam type femoroacetabular impingement observed with especially young athletic males. An abnormal shape is observed in the femoral head (prominent bony structure at the anterolateral femoral head and neck). Shearing forces lead to chondral or labral mower injury especially during flexion of the hip joint. Pincer type is seen with middle-aged women. This type shows abnormal shape of the acetabulum. Anterior acetabular overcoverage (acetabular retroversion), protrusion of the acetabulum, coxa profunda is shown to cause. Magnetic resonance imaging demonstrates abnormal anatomical findings of cam or pincer type femoroacetabular impingement, as well as findings of osteoarthritis such as the joint space narrowing, osteophytes, subchondral sclerosis and cysts.

Keyword: Anatomy, femoroacetabular impingement, magnetic resonance imaging

\section{P-80 \\ Carpal bones: three-dimensional reconstruction, photorealistic imaging and physical biomodeling}

Sunar $M^{*}$, Kapakin $S^{* *}$, Durur Subaşı $\mathrm{I}^{* * *}$

*Department of Anatomy, Faculty of Medicine, Erzincan University, Erzincan, Turkey; **Department of Anatomy, Faculty of Medicine, Ataturk University, Erzurum, Turkey; ***Department of Radiology, Faculty of Medicine, Ataturk University, Erzurum, Turkey

The goal of this study is to create tangible hard copies of the carpal bones with rapid prototyping and use them in surgical applications. The Surfdriver software package is applied to the images obtained from different imaging modalities to reconstruct the carpal bones as three-dimensional DXF (data exchange file) models. These models are post-processed in Cinema 4D software for virtual reality modelling language (VRML) and STL (Standard Triangulation Language) formats. Solid replicas are manufactured in a rapid prototyping machine by using the STL format. Surgeons can rehearse the surgical procedures by using custom made replicas. They can be ready for complex situations of surgery in advance and prevent unpredictable adverse events in the surgery.

Keywords: Carpal bones, photorealistic imaging, reconstruction 


\section{P-81}

The protective effect of intravenous lipid emulsion treatment against diazinon induced renal injury: an experimental study

Ayan $\mathrm{M}^{*}$, Uysal $\mathrm{M}^{* *}$, Tas $\mathrm{U}^{* *}$, Irmak Sapmaz $\mathrm{H}^{* * *}$, Bicakci $\mathrm{H}^{* * * *}$, Sarsilmaz $\mathrm{M}^{* * * *}$

*Department of Emergency Medicine, Faculty of Medicine, Gaziosmanpasa University, Tokat, Turkey; **Department of Anatomy, Faculty of Medicine, Gaziosmanpasa University, Tokat, Turkey; $* * *$ Department of Anatomy, Faculty of Medicine, Inonu University, Malatya, Turkey; $* * * *$ Department of Anatomy, Faculty of Medicine, Sifa University, Izmir, Turkey

Objectives: Diazinon (DZN) is a lipid soluble synthetic organophosphorus insecticide widely used in agricultural and household applications. DZN exposure occurs through inhalation, ingestion and dermal contact. Intravenous lipid emulsion (ILE) has been shown to reduce toxicity caused by some lipid soluble drugs. In this study, we evaluated the protective effect of intravenous lipid emulsion treatment on the kidney tissue in diazinon intoxicity.

Methods: 21 male Wistar albino rats, (weighing 180-200 g) randomly divided into three equal groups. Groups organized as; Group I control, Group II diazinon, Group III diazinon + lipid emulsion treatment. Group I, only $1 \mathrm{ml}$ corn oil was given by gavage. Group II, $335 \mathrm{mg} / \mathrm{kg}$ diazinon were given by gavage. Group III, in addition to diazinon 20\% lipid solution (3 $\mathrm{ml} / \mathrm{kg}$ ) were administered via tail vein into rats. At the end of the experimental period, animals were killed by exsanguination under ketamine/xylasine anesthesia. Kidney tissues removed and 5 micrometer histological sections were taken. Tissue sections were examined for histopathological changes by Hematoxylin and eosin staining and for apoptotic changes by Caspase-3 immunohistochemical staining.

Results: An increase of Caspase- 3 immunoreactivity was observed in the diazinon group when compared with the control group. Additionally, a decrease of Caspase-3 immunoreactivity was observed in the diazinon + lipid emulsion treatment group.

Conclusion: The findings from our study suggest that lipid emulsion treatment has protective effect on diazinon induced kidney toxicity.

Keywords: Diazinon, toxicity, apoptosis, kidney

\section{P-82}

\section{Thyroid gland hemiagenesis}

Uysal $M^{*}$, Hasbek $Z^{* *}$, Tas $U^{*}$, Meydan $S^{* * *}$

*Department of Anatomy, Faculty of Medicine, Gaziosmanpasa University, Tokat, Turkey; **Department of Nuclear Medicine, Faculty of Medicine, Cumhuriyet University, Sivas, Turkey; $* * *$ Department of Anatomy, Faculty of Medicine, Sifa University, Izmir, Turkey

Thyroid hemiagenesis (TH) is a developmental disorder of the thyroid gland, it is common in women and often it shaped left lobe agenesis. TH is a rare congenital anomaly and it is the result for one of thyroid lobe development disruption. Prevalence among adolescents was founded to be $0.2-0.05 \%$. Often, TH may be associated with other thyroid diseases that Grave's disease, Hashimoto's thyroiditis, subacute thyroiditis, simple goiter, toxic adenoma, carcinoma, colloidal nodules, such as parathyroid hyperplasia. Thyroid ultrasound is not detected in the affected lobe. Thyroid scintigraphy is not visible radiopharmaceutical uptake in thyroid region. This report is intended for draw attention to TH cases which uncommon but clinical follow-up necessary.

Case report: 43 -year-old female patient is followed due to impaired thyroid function tests. Thyroid scintigraphy with technetium-99m pertechnetate; the right lobe uptake was normally, the left lobe the uptake was detected. The case was confirmed by thyroid ultrasound. TH common in women (3: 1 ratio) and is mostly in the form of agenesis of the left lobe (4:1 ratio). Half of the patients do not have isthmus. Thyroid scintigraphy is a method commonly used in the evaluation of patients with abnormal thyroid function. However, thyroid scintigraphy gives incorrect results for especially hyperfunction nodule, thyroiditis, carcinoma, atrophy, or display errors conditions. Thus ultrasound and other radiological imaging techniques should be used for the differential diagnosis.

Keywords: Thyroid development, hemiagenesis, thyroid scintigraphy

\section{P-83}

\section{An interesting case: Ramsay Hunt syndrome}

Uysal $M^{*}$, Ayan $M^{* *}$, Somuk BT ${ }^{* * *}$, Esen $M^{* *}$, Tas $U^{*}$

*Department of Anatomy, Faculty of Medicine, Gaziosmanpasa University, Tokat, Turkey; ${ }^{*}$ Department of Emergency Medicine, Faculty of Medicine, Gaziosmanpasa University, Tokat, Turkey; $* *$ Department of Otolaryngology, Faculty of Medicine, Gaziosmanpasa University, Tokat, Turkey

The Ramsay Hunt syndrome (RHS) is a clinical situation which occurs of the reactivation of varicella zoster virus and can be resulting in facial nerve involvement. Severe ear pain, rash vesicular outer ear, facial nerve paresis in tracing acute paralysis and redness are often seen in RHS patients. In this case report, 78 years old male patient with redness and vesicular rash on the left side of the neck and at the left auricle, admitted to the Emergency Department of Gaziosmanpasa University is presented. The patient was admitted to another hospital for skin lesions and eczema treatment is started. But when because of dermatological lesions become painful, patient was admitted to our emergency department of our faculty. Despite the absence of facial nerve paralysis, RHS was kept in mind and antiviral therapy was started by our department. Due to the development of facial nerve paralysis, the patient was admitted to the emergency service at the next day again, and hospitalization of the patient to the Ear, Nose and Throat disease service is provided. RHS is a serious clinical condition which can affect the patient's quality of life, and antiviral therapy and symptomatic treatment for patients 
should be started within the shortest possible time. If the duration of treatment is delayed, complications and morbidity rates are increasing. As in our case, not accompanied by facial nerve paralysis RHS cases, the presence of the syndrome skipping the initial period of treatment may be delayed. In cases of facial nerve paralysis is accompanied, we believe that the existence of this syndrome to be kept in mind by clinician.

Keywords: Facial nerve palsy, Ramsay Hunt syndrome, vesicular rash

\section{P-84}

\section{Osteitis pubis with a case report}

Gul SS*, Uysal $M^{* *}$, Hasbek $Z^{* * *}$, Çiçek $M^{* *}$

*Department of Nuclear Medicine, Faculty of Medicine, Gaziosmanpasa University, Tokat, Turkey; **Department of Anatomy, Faculty of Medicine, Gaziosmanpasa University, Tokat, Turkey;

***Department of Nuclear Medicine, Faculty of Medicine, Cumhuriyet University, Sivas, Turkey

Osteitis pubis that cause of the pelvic pain has been known as a noninfectious inflammation of the pubic bone. The prevalence has been well studied in athletes, though the prevalence in the community is unknown. Patients are usually suffering from progressive type suprapubic pain radiating to the groin. Diagnosis of osteitis pubis is usually made clinically but it is confirmed by magnetic resonance imaging, pelvic radiography and bone scintigraphy. In this case report, we present a case of osteitis pubis. A whole body three-phase bone scintigraphy with Technetium-99m methylenediphosphonate was performed to female patient with pain in the pelvic region. Threephase bone scintigraphy showed that "Bloody and blood pool phase images confirms the increase of perfusion and hyperemia in the region of the symphysis pubis, and the presence of increased focal osteoblastic uptake in the right part of the region of the symphysis pubis". These findings were evaluated in accordance with osteitis pubis. Osteitis pubis especially is seen in athletes engaged in football, ice hockey and basketball sports that are based on the sudden and very repetitive activities. In contrast to the literature, our case was a housewife. The use of bone scintigraphy during diagnosis will guide the clinician in the patient population which osteitis pubis is seen less often.

Keywords: Osteitis pubis, bone scintigraphy, symphysis pubis

\section{P-85}

Associations among recurrent laryngeal nerve, inferior horn of thyroid cartilage, Berry's ligament and Zuckerkandl's tubercle

Kastan OZ*, Calguner E**, Agirdır BV ${ }^{* * *}$, Sindel $M^{* * * *}$

*Akdeniz University Faculty of Nursing, Antalya, Turkey; **Department of Anatomy, Faculty of Medicine, Gazi University Ankara, Turkey; $* * *$ Department of Otorhinolaryngology, Faculty of Medicine, Akdeniz University Antalya, Turkey; $* * * *$ Department of Anatomy, Faculty of Medicine, Akdeniz University, Antalya, Turkey
During neck surgery, localizing and monitoring the nerve has become "sine qua non" rule in order to avoid damaging recurrent laryngeal nerve. Any anatomical structure should not be cut without seeing the nerve, single landmark should not be relied upon and branching differences should be taken into consideration. During the neck surgery; Zuckerkandl's tubercle, Berry's ligament, the inferior horn of thyroid cartilages has become crucial anatomical landmarks in order to protect the integrity of the recurrent laryngeal nerve. In this study, sixty sides of thirty male postmortem human cadavers obtained from Department of Anatomy, Akdeniz University was used. The proximal part of the recurrent laryngeal nerve, before the inferior thyroid artery arises from its source has been observed in 52 sides $(87 \%)$ inside the tracheo-esophageal groove and in 8 sides $(13 \%)$ running laterally to the trachea. The recurrent laryngeal nerve was encountered passing behind and through the branches of the inferior thyroid artery in 55 sides $(92 \%)$ and $6(8 \%)$ respectively. At all sides; the nerve was piercing the larynx $0.6 \pm 0.1 \mathrm{~mm}$ below the inferior horn of thyroid cartilage, passing next to the inner-lower side of Berry's ligament and running under the lower middle part of Zuckerkandl's tubercle. In conclusion, these landmarks and their upper mentioned distances to the laryngeal nerve can be taken into consideration as important surgical guides.

Keywords: N. laryngeus recurrens, Cartilago thyroidea, Berry ligamenti, Zuckerkandl tüberkülü

\section{P-86}

\section{Analysis of the thymus using MDCT imaging in adults}

Yilmaz $N^{*}$, Unver Dogan $\mathrm{N}^{*}$, Sivri $\mathrm{M}^{* *}$, Dogan $\mathrm{KH}^{* * *}$, Ozbek $\mathrm{S}^{* *}$

*Department of Anatomy, Faculty of Medicine, Selcuk University, Konya, Turkey; **Department of Radiology, Faculty of Medicine, Selcuk University, Konya, Turkey; ***Department of Forensic Science, Faculty of Medicine, Selcuk University, Konya, Turkey

The thymus is a lymphoid organ that has many immunologic functions, primarily $\mathrm{T}$-cell differentiation. The thymus varies in size, shape and density between individuals. It was aimed to quantify CT characteristics of the normal thymus in an adult population in this study. Data obtained analysis of MDCT (Multidetector Computed Tomography) (Siemens Somatom Sensation, Erlanger, Germany, 2005) images of 15 males (mean age $49.20 \pm 4.65$ ), 15 females (mean age $49.93 \pm 5.36$ ), total 30 (mean age $49.56 \pm 19.12$ ) adults which have not any thymus malformation in Selcuk University Department of Radiology in 2013-2014. Categorizing the shape of the thymus proved somewhat difficult. In many patients the gland had a complex shape, which could be described as arrowhead, bilobal or rounded. Thymus was mainly arrowhead shape (93\%) in males, $47 \%$ arrowhead, $47 \%$ rounded and $6 \%$ bilobal in females. Thymus was at right in 4, at left in 1 and at middle line in 10 of males. It was at left in 3 and at middle line in 12 of females. Thymus was mainly located at T4-5 vertebral level (40\%). Level of thymus, densities and vertical dimensions of thymus were not significant- 
ly different, but transverse dimensions and types were significantly different when compared between sexes. Fat ratio of thymus undergoes change by age. Comprehensive studies describing the normal thymus size and morphology are few in the literature. As MDCT images clearly define the appearance of the thymus, they are especially used in the routine by radiologists to separate normal changes and abnormal structures.

Keywords: Thymus, MDCT, morphology

\section{P-87}

\section{The right aortic arch and aberrant left subclavian} artery: a case report

Unver Dogan $\mathrm{N}^{*}$, Fazliogullari $\mathrm{Z}^{*}$, Uysal II*, Karabulut $\mathrm{AK}^{*}$, Nayman $\mathrm{A}^{* *}$

*Department of Anatomy, Faculty of Medicine, Selcuk University, Konya, Turkey; ${ }^{* *}$ Department of Radiology, Faculty of Medicine, Selcuk University, Konya, Turkey

Anomalies of the aortic arch and its main branches are rare: estimations from autopsy series indicate a frequency of $3 \%$. The most common finding is a left aortic arch accompanied by an aberrant right subclavian artery. One of the less common anomalies is the occurrence of a right aortic arch associated with an aberrant left subclavian artery. In a 45-year-old male patient's thorax computed tomography (CT), at Selcuk University Department of Radiology variation of the branches of aortic arch was observed. Brachiocephalic trunk was absent, arcus aorta was at right and left subclavian artery was passing behind esophagus aberrantly in the case. There were four branches of the arch of the aorta. These branches were the left common carotid artery, the right common carotid artery, the right subclavian artery and the left subclavian artery respectively. There wasn't any other major vascular anomaly in the patient. Approximately $0.1 \%$ of population has a right sided aortic arch, and about half of these have an aberrant left subclavian artery. Although an aberrant left subclavian artery may occur in isolation, it is the commonest anomaly associated with a right aortic arch. This variation is generally asymptomatic but in some cases, compression of the esophagus or trachea may occur if its origin is dilated. In these cases, it may present with either stridor or dysphagia. Aberrant arteries' significance is set forth in the radiological and surgical literature. The importance of thorax CT in determining the vascular anatomy and its variations was emphasized with this case.

Keywords: Right aortic arch, variation, aberrant left subclavian artery

\section{P-88}

Foot anthropometry in employees working at air transportation ground services

Kilic N, Durgun B

Department of Anatomy, Faculty of Medicine, Çukurova University, Adana
Objectives: The knowledge of spesific population standards and measurements relating to the foot is important for all shoe design since the foot measurements varies according to age, gender and race. The aim of this study is to obtain the data related to the foot in employees working at air transportation ground services, which has never been determined before.

Methods: In this study, foot measurements were obtained from 35 employees who work at air transportation ground services (21 male and 14 female) aged between 16-55 years. Measurements were performed by using tape measure and anthropometric set. These measurements are the foot length, truncated foot length, height of medial longitudinal arch, foot breadth, bimalleolar breadth and bimalleolar circumference.

Results: The study group included 21 male (\%60) and 14 female (\%40). For total, mean age was $34.2 \pm 8$ years, mean height is $170.4 \pm 7.7 \mathrm{~cm}$ and mean weight is $76.3 \pm 18.4 \mathrm{~kg}$. The mean right and left foot lengths were $25.6 \pm 1.9 \mathrm{~cm}$, right and left truncated foot lengths were $18.5 \pm 1.4 \mathrm{~cm}$. Heights of medial longitudinal arches were $2.0 \pm 0.6 \mathrm{~cm}$ for right foot and $2 \pm 0.5$ $\mathrm{cm}$ for left foot. The mean foot breadth at the narrowest part were $8.7 \pm 1.1 \mathrm{~cm}$ for right foot, $8.6 \pm 1.0 \mathrm{~cm}$ for left foot and the mean foot breadth at the widest part were $9.9 \pm 0.9 \mathrm{~cm}$ for right foot, $9.9 \pm 0.8 \mathrm{~cm}$ for left foot. The mean of bimalleolar breadth were $7.0 \pm 0.7 \mathrm{~cm}$ for right foot and $7.1 \pm 0.7 \mathrm{~cm}$ for left foot. The mean of bimalleolar circumference were $25.7 \pm 2.3$ for right foot and 25.7 \pm 2.6 for left foot.

Conclusion: The results of this study were expected to help for determining spesific population standards for the design of shoes and then to provide foot comfort and health for employees who work at air transportation ground services industry which has millions of employees and long working times.

Keywords: Foot, foot anthropometry, foot dimensions, air transportation

\section{P-89}

The arterial blood supply of the pancreas: an anatomical and surgical study

Kocabiyik N*, Ozsoy S**, Baykal B***, Zeybek N ${ }^{* * * *}$, Yazar F*, Karapirli $\mathrm{M}^{* * * * *}$, Korkusuz I $\mathrm{I}^{\star * * * *}$

*Department of Anatomy, Gulhane Military Medical Faculty, Ankara, Turkey; ${ }^{*}$ Department of Forensic Medicine, Gulhane Military Medical Faculty, Ankara, Turkey; ***Department of Histology and Embryology, Gulhane Military Medical Faculty, Ankara, Turkey; $* * * \star$ Department of General Surgery, Gulhane Military Medical Faculty, Ankara, Turkey; $* * * * *$ Forensic Medicine Institution Ankara Group Presidency, Ankara, Turkey; $* * * * * *$ Forensic Medicine Institution Ankara Group Presidency Morgue Specialty Department, Ankara, Turkey

There is a controversy in the literature regarding definition or interpretation of pancreatic arteries. This study is planned to study the results of studies, which were previously performed by analyzing wide radiological series, on cadavers. All of the important pancreaticoduodenal arterial, venous and nervous arches are localized between the fusion fascie of Treitz an pancreatic parenchyma. The arterial supply of the head and collum of pancreas is from anterior, intermediate, posterior pancreati- 
coduodenal and prepancreatic arterial arches. That, the head and collum of the pancreas are reported to be supplied from minor resources in the literature in addition to pancreaticoduodenal arterial arches and there is still controversy among the authors, made us think that there is still a need for detailed examination of the vessels of the pancreas in anatomical and surgical aspects. This study is performed on 60 formaline fixed pancreas and surrounding tissues. Superior pancreaticoduodenal artery arising from gastroduodenal artery, inferior pancreaticoduodenal artery arising from superior mesenteric artery and dorsal pancreatic artery, splenic artery and their branches are tracked and measurements regarding pancreas morphology are performed. Variational situations observed other than classical arterial supply or coming from a different source are recorded. Acquired results are compared to the results in the literature. This study may yield a new perspective to the arguments on the blood supply of the pancreas, acquired results may reveal the variational differences in the arteries that directly supply the pancreas and these data may contribute to surgery during pancreatic resections.

Keywords: Pancreas, arterial blood supply, surgery

\section{P-90}

Morphometric evaluation of the lunate fossa (a study on clinical and anatomical lunate fossa)

Onder $\mathrm{M}^{*}$, Aldemir $\mathrm{C}^{* *}$, Oguz Yolcular B**, Oguz $\mathrm{N}^{*}$

*Department of Anatomy, Faculty of Medicine, Akdeniz University, Antalya, Turkey; ${ }^{* *}$ Clinic of Orthopaedics and Traumatology, Antalya Education and Research Hospital, Antalya, Turkey; ${ }^{* *}$ Department Of Biostatistic and Medical Informatics, Akdeniz University Faculty of Medicine, Antalya, Turkey

Objectives: Distal radius fractures are the most common clinical cases. In the treatment of distal radius fractures commonly used method is the application of volar plate. One of the problems commonly encountered by surgeons in volar plate application is to send the screw plate to the joint space. To minimize this problem, depth of lunate fossa should be known. Furthertmore, transverse and sagittal diameter of lunate fossa and width of radius should be kept in mind.

Methods: In our study, we used 25 left and 25 right radius from Department Of Anatomy Laboratory, Akdeniz University Faculty Of Medicine, Antalya. The distances depth, transverse and sagittal diameter of lunate fossa and width of radius were measured with MicroscribeG2X. Data were processed and analyzed with SPSS (Statistical Package for Social Sciences) version 12.0. Descriptive analysis was applied to dataset and the results were presented as mean \pm standard deviation. Independent Samples T test was used for comparison of quantitative variables with normal distribution. Data were processed and analyzed with SPSS (Statistical Package for Social Sciences) version 13.0 and a p-value greater than 0.05 was considered statistically significant.

Results: According to measurements of anatomical structures at left and right distal radius means and standard deviations were: The depth of lunate fossa: on the left $2.41 \mathrm{~mm} \pm 0.52 / \mathrm{on}$ the right $2.53 \mathrm{~mm} \pm 0.79$. The sagittal diameter of lunate fossa: on the left $19.66 \mathrm{~mm} \pm 1.62 /$ on the right $18.78 \mathrm{~mm} \pm 1.64$. The transverse of lunate fossa: on the left $11.26 \mathrm{~mm} \pm 0.84$ /on the right $11.08 \mathrm{~mm} \pm 0.86$, respectively. There were no statistically significant differences between the left and right depth of lunate fossa, the sagittal diameter of lunate fossa and transverse of lunate fossa $(\mathrm{p}=0.448, \mathrm{p}=0.060, \mathrm{p}=0.531)$.

Conclusion: We consider that these measurements should be kept in mind in volar plate application to the distal radius is sending the screw through the joint space.

Keywords: morphometry, volar plate, distal Radius, lunate surface, lunate fossa, carpal articular surface, population of Turkish

\section{P-91}

\section{Location and incidence of zygomaticotemporal foramen, supraorbital and supratrochlear foramen or notch in skulls}

\author{
Beger O, Gilan IY, Aktekin M \\ Department of Anatomy, Faculty of Medicine, Mersin University, \\ Mersin, Turkey
}

Objectives: It was aimed to obtain detailed knowledge about the location and incidence of the zygomaticotemporal foramen (ZTF), supraorbital (FSO) and supratrochlear (FST) foramina or supraorbital (ISO) and supratrochlear (IST) notches in skull.

Methods: Distances of FSO/ISO and FST/IST to midline were measured in 29 dry skulls. In 35 skulls, FZTs which were located on the temporal surface of the zygomatic bone were classified in 3 groups regarding its location as on the level of zygomatic arch (AZS), below the level of zygomatic arch (AZAS), and on the frontal process $(\mathrm{PF})$.All measurements were performed with digital caliper. Data were analyzed statistically.

Results: The total numbers of FZTs were 160 and those were found on PF, AZS and AZAS with the ratios of $52 \%, 38 \%$ and $10 \%$, respectively. Widths of ZTFs were so small that it was impossible to measure with digital caliper. Only 8 (5\%) FZTs entered from the temporal surface and exited from the lateral surface of zygomatic bone. No statistically significant difference was detected regarding the number of foramina in either side, so the results were given in total. A single foramen was observed in $16 \%$ of the sides while 2 foramina in $31 \%, 3$ in $24 \%, 4$ in $22 \%$, 5 in $5 \%$, and 6 in $2 \%$ of the sides. The average distances of FSO, ISO, FST or IST to midline were measured as $28.53 \pm 1.04 \mathrm{~mm}$, $22.70 \pm 1.24 \mathrm{~mm}, 21.22 \pm 1.15 \mathrm{~mm}$ or $21.33 \pm 0.49 \mathrm{~mm}$, respectively. Double FSO was observed in $14 \%$ of sides while double FST was observed in $3 \%$. The frequency of bilateral FSO, ISO, FST or IST in the skulls was $62 \%, 3 \%, 24 \%$ and $13 \%$, respectively.

Conclusion: The nerves passing through the foramina which were the regions of interest in this study play role in migraine genesis and surgical decompression or chemodenervation of them can aid in alleviating migraine headache symptoms. Precise knowledge about the anatomy of these foramina will provide more effective results in surgical decompression.

Keywords: Zygomaticotemporal foramen, supraorbital and supratrochlear foramen/notch, nerve decompression, migraine treatment 


\section{P-92}

\section{Case of accessory and duplicated arteria hepatica variations}

Turamanlar O*, Kacar E* ${ }^{* *}$ Cartıll $\mathrm{O}^{*}$, Akbal I*

*Department of Anatomy, Faculty of Medicine, Afyon Kocatepe University, Afyonkarahisar, Turkey; ${ }^{* *}$ Department of Radiology, Faculty of Medicine, Afyon Kocatepe University, Afyonkarahisar, Turkey; ${ }^{* * *}$ Atatürk Vocational School df Health Services, Afyon Kocatepe Universty, Afyonkarahisar, Turkey

Objectives: Hepatic artery that branch of truncus coeliacus is examined in two parts. Common hepatic artery extends up from truncus coeliacus to the exit point of gastroduodenal artery. Hepatic artery proper extends up from the starting point of gastroduodenal artery to the exited the point of bifurcation right and left branches. Apart from these classical branching, quite different variants of hepatic artery have been identified. About this case, we aimed to present a rare variation of hepatic artery proper.

Case report: In multiphasic abdominal CT scans of 62 year old male patient it was detected that, left gastric artery originating from left hepatic artery and dublication of right hepatic artery originate from, proximal part of superior mesenteric artery and coeliac trunk.

Conclusion: Hepatic artery variations rate is observed 40$45 \%$. Accessories or displaced right hepatic artery generally originate from superior mesenteric artery and accessories or displaced left hepatic artery generally exit from left gastric artery. Like our case, left hepatic artery arise from left gastric artery and right hepatic artery arise from superior mesenteric artery variations encountered nearly $0.5-2 \%$. In our case accessory right hepatic artery variation found in $1 \%$. Association of these variations in our case, not only reduce the incidence in the literature but also increases originality. In recent years, modern surgery and transplantation procedures, take into account hepatic artery anomalies so the these issues importance and value increase. Thus, we believe that our case will gain a new perspective to clicians about the strategies for diagnosis and treatment methodologies of patients have variations like our case.

Keywords: Variation, hepatic artery, CT

\section{P-93}

Morphometric measurements of proximal and distal humerus segments

$\underline{\mathrm{Al} O}$, Atay E, Ertekin T, Nisari M, Ulger H

*Department of Anatomy, Faculty of Medicine, Erciyes University, Kayseri, Turkey

The humerus is longest and largest bone in the upper limb. In addition, knowing the length of the humerus segments is important for orthopedic surgery and forensic medicine. For this purpose, humeral length $(\mathrm{HU})$ and some morphometric parameters in proximal and distal segments are measured in 30
(15 right, 15 left) adult dry humerus that is gender unknown. In this study; the mean distances between the articular segment of the humeral head and the greater tuberosity (A), the mean distances between humeral head and collum anatomicum (B), the mean distances between proximal and distal point of olecrane fossa $(\mathrm{C})$, the mean distances between distal part of olecrane process and trochlea of humerus (D), the mean distances between proximal edge of olecrane fossa and distal part of trochlea of humerus (E), maximum horizontal distances between two points of greater tubercule $(\mathrm{F})$, maximum horizontal distances between two points of lesser tubercule $(G)$, maximum horizontal distances between most prominent point on lesser tubercule to midpoint on greater tubercule $(\mathrm{H})$, maximum distances between the medial and the lateral epicondyles (J), maximum distances between the medial and the lateral end of trochlea at the anterior surface $(\mathrm{K})$, maximum thickness at most concave part of trochlea (L) parameters were measured by digital caliper. The measurements were evaluated with SPSS 21 statistical software package. Average HU was calculated as $307.3 \pm 21.5 \mathrm{~mm}$ in the right humerus, $303.5 \pm 16.3 \mathrm{~mm}$ in the left humerus. Statistically significant correlation was found between $\mathrm{HU}$ and $\mathrm{D}, \mathrm{E}, \mathrm{F}, \mathrm{H}, \mathrm{J}, \mathrm{K}, \mathrm{L}$ parameters in right humerus; B, F, J, K parameters in the left humerus. As a result; it is expected that the humerus length measurements give an idea about humeral segments and all of these measurements would be able to support anthropological, archaeological, orthopedic and forensic works.

Keywords: Humerus, segments, morphometry

\section{P-94}

\section{Volume calculation of frontal lobe structures in Parkinson disease by using DiffeoMap software}

Palanci $\mathrm{O}^{*}$, Ocak H${ }^{* *}$, Ozyasar A F**, Kalaycioglu A**, Acer $\mathrm{N}^{* * *}$ *Department of Anatomy, Bayburt Vocational School of Health Services, Bayburt University, Bayburt, Turkey; **Department of Anatomy, Faculty of Medicine, Karadeniz Technical University, Trabzon, Turkey; ***Department of Anatomy, Faculty of Medicine, Erciyes University, Kayseri, Turkey

Parkinson disease is a neurodegenerative illness which is characterized by some symptoms such as tremor, rigidity and bradykinesia. Atrophy is a good marker of this disease. In this study we aimed to compare frontal lobe volumes of 5 Parkinson disease patients with aged matched 5 control people who have no histroy of any neurological, psychiatric or cognitive sufferings. Mr scans, obtained from these participants, were analyzed with DiffeoMap software. MRI is a valuable tool for Parkinson disease since it reveals atrophy cases related with the disease and accelerates diagnosis stage. Results of this study might be useful for evaluation of Parkinson disease patients. More studies are required with larger data for supporting results of these type of studies.

Keywords: Parkinson, diffeomap, frontal lobe 


\section{P-95}

Plexus brachialis comprising of fasciculus anterior (?) and posterior: a case report

Elvan $\mathrm{O}^{*}$, Aytac $\mathrm{G}^{* *}$, Bobuş Kara $\mathrm{A}^{*}$

*Department of Anatomy, Faculty of Medicine, Mersin University, Mersin, Turkey; **Department of Anatomy, Faculty of Medicine, Akdeniz University, Antalya, Turkey

During the dissections performed in a group of fetuses, we encountered an unusual branching pattern of the brachial plexus, on the left side of a 32 week -age male fetus. The brachial plexus in this case was formed by the union of the anterior rami of the 5 th cervical- 1 st thoracal spinal nerves. But, the anterior rami of the $\mathrm{C} 5,6$ and 7 joined to form a trunk (Tr1) while, the anterior rami of the C7, 8 and a branch of T1 joined to form another trunk ( $\operatorname{Tr} 2)$. Then these two trunks ( $\operatorname{Tr} 1$ and $\operatorname{Tr} 2)$ meet to form a fasciculus that can be called as fasciculus anterior (?). This variative fasciculus, gave off musculocutaneus, median and ulnar nerves in this case which had no lateral and medial fasciculi. The posterior fasciculus was formed by the union of a branch of $\mathrm{C} 5$, and $\operatorname{Tr} 1$ and two branches from $\operatorname{Tr} 2$. Despite the formation of this variative posterior fasciculus, there was no abnormality in its course and branching pattern.

Keywords: Plexus brachialis, branching pattern, fetus, variation

\section{P-96}

The effectivity of whole body bone scintigraphy for the diagnosis and follow-up of reflex sympathetic dystrophy

Gül SS*, Uysal M**, Çiftçi ÖD*, Meydan S***, Taş U**

*Department of Nuclear Medicine, Faculty of Medicine, Gaziosmanpaşa University, Tokat, Turkey; ${ }^{*}$ Department of Anatomy, Faculty of Medicine, Gaziosmanpaşa University, Tokat, Turkey; ${ }^{* *}$ Department of Anatomy, Faculty of Medicine, Şifa University, Izmir, Turkey

Reflex Sympathetic Dystrophy (RSD) is a clinical syndrome that characterized with distal extremity pain, edema and trophic changes, besides vasomotor instability, this disease's pathophysiology has not been fully elucidated. This disease usually occurs following trauma at hands and feet. Although imaging techniques help in the diagnosis of this disease, studies have been showed that valuable of three-phase bone scintigraphy in the early diagnosis. In this report, it has been presented three cases with clinically diagnosed RSD, and it has been emphasized three-phase bone scintigraphy with Technetium-99m methylenediphosphonate (TVC) to confirm the diagnosis and to determining the success of treatment. Early diagnosis and treatment of RSD is very important for determining the prognosis. In particular, for suspected cases that clinic is not fully seated, three-phase bone scintigraphy is very important in the early stages of RSD.

Keywords: Reflex sympathetic dystrophy, vasomotor instability, bone scintigraphy

\section{P-97}

Renal ptosis case diagnosed with renal scintigraphy

Gül SS*, Uysal M**, Koçyiğgit Deveci E ${ }^{* * *}$ Meydan $M^{* * * *}$, Taş $\mathrm{U}^{* *}$

*Department of Nuclear Medicine, Faculty of Medicine, Gaziosmanpaşa University, Tokat, Turkey; **Department of Anatomy, Faculty of Medicine, Gaziosmanpaşa University, Tokat, Turkey; ${ }^{* *}$ Department of Nuclear Medicine, Adana Numune Research and Training Hospital, Adana, Turkey; ${ }^{* * *}$ Department of Anatomy, Faculty of Medicine, Şifa University, Izmir

Renal ptosis (RP) is an abnormal condition in which the kidney drops down into the pelvis. It is believed to result from deficiency of supporting perirenal fasciae. RP is asymptomatic in most patients. However, RP can be characterized by violent attacks of colicky flank pain, nausea, hematuria and proteinuria. Diagnosis is contemplated based upon patient symptoms. Diagnosis is confirmed by intravenous urography and renal DMSA. In this report a patient with renal ptosis diagnosed after the scintigraphy is presented.

Case report: A female patient admitted to our hospital because of abdominal pain, was referred to the nuclear medicine department. It is seen that excretion and concentration functions of the left kidney were found to be at normal levels by the dynamic renal scintigraphy made by Technetium- $99 \mathrm{~m}$ with diethylene triamine pentaacetic acid to the patient. It also observed that the right kidney is not in the expected anatomic location, it is at the upper part of the bladder and close to the anterior segment as ptotic. Information of bladder function could not be obtained due to the superposition. In a separate session with Technetium99m dimercaptosuccinic acid static renal scintigraphy, dilatation in the right kidney compatible with hypoactive areas monitored observed but renal cortical scarring did not detected. Participation in the global renal function was calculated as $41 \%$ for the right kidney, 59\% for left kidney. Because of clinical findings are unclear in patients with renal ptosis, imaging has a great of importance. Renal scintigraphy is thought to be more important in these patients because of determining the both of renal localization and renal functions.

Keywords: Renal ptosis, renal cortical scintigraphy, technetium-99m

\section{P-98}

The investigation of relationship between head-neck anthropometric measurements and balance analysis

Uluçam E, Çıkmaz S, Yılmaz A, Parlak M, Dönmez D, Karahan $M$

Departmant of Anatomy, Faculty of Medicine, Trakya University, Edirne, Turkey

Objectives: The relationship of some head-neck anthropometric measurements with the data obtained from balance analysis is aimed to reveal.

Methods: This study was participated 15 healthy male volunteers. Subjects were measured at the same time of day (10:00- 
12:00). Cranio corpo graphy section of the CMS20P-2 (Zebris ${ }^{\circ}$ Medical GmbH, Germany) was used for measurements. The subjects of the head length, head circumference, head width, neck circumference, neck width and length anterior-posterior diameter were measured. As the balance values, the longitudinal deviation, lateral sway width, angular deviation, self spin, longitudinal sway, lateral sway, angle of torticollis, were evaluated. The relationships between data were compared statistically.

Results: The head length showed moderate correlation with lateral sway width $(r=-0.29)$, self spin $(r=-0.35)$ and lateral sway $(r=0.28)$. A moderate positive relationship between the head length and longitudinal deviation was found. Both neck circumference and neck width a moderate correlation with longitudinal sway was found. Neck circumference and neck width values showed a moderate correlation with longitudinal sway ( $\mathrm{r}=0.46$ and $\mathrm{r}=0.36$ ).

Conclusion: In our study, between the balance and the headneck anthropometry showed that the correlation is moderate.

Keywords: Balance, anthropometry, head, neck.

\section{P-99}

Sever's disease: calcaneal apofizitis

Uysal M*, Gül SS ${ }^{* *}$, Hasbek $Z^{* * *}$, Koçyiğit Deveci E****, Taş U* *Department of Anatomy, Faculty of Medicine, Gaziosmanpaşa University, Tokat, Turkey; ${ }^{*}$ Department of Nuclear Medicine, Faculty of Medicine, Gaziosmanpaşa University, Tokat, Turkey; ${ }^{* *}$ Department of Nuclear Medicine, Faculty of Medicine, Cumhuriyet University, Sivas, Turkey; $* * * *$ Department of Nuclear Medicine, Adana Numune Research and Training Hospital, Adana, Turkey

Calcaneal apophysitis is the most common cause of heel pain in children and defined as Sever's disease. Typically, it defines in the rapid growth phase of children and it appears at the beginning of exercise season. Disease is particularly prevalent in adolescents and children those 8-15 years of age, very active, athletic and overweight. In a child presenting with heel pain should be considered for Achilles tendinitis, retrocalcaneal bursitis, calcaneal stress fractures, calcaneal cysts, osteomyelitis, and plantar fasciitis in the differential diagnosis. Although Sever's Disease is a common clinical presentation, it is very little document in the national and international literature about Sever's Disease. In this report, it presented a case with Sever's Disease in order to contribute to the literature.

Case report: Male patients with bilateral heel pain were performed three phase whole-body scintigraphy with technetium99m methylenediphosphonate: "Perfusion and blood pool images showed hyperemia in calacaneus with both feet areas. Late-phase static images viewed increased Osteoblastic activity involvement in bilateral symmetrical tuber calcanei as focal style. Patient was been evaluated Sever's Disease as a result of clinical and scintigraphic findings and conservative treatment was started. Foot x-rays are usually normal in the early stages of Sever's Disease. Thus, three-phase bone scintigraphy may be useful especially in cases where the diagnosis is suspected.

Keywords: Sever's disease, calcaneal apophysitis, whole body bone scintigraphy

\section{P-100}

\section{A rare variation of posterior cord branching pattern Dursun A, Bilkay C, Albay S \\ Departmant of Anatomy, Faculty of Medicine, Suleyman Demirel University, Isparta, Turkey}

Variations of the brachial plexus are not uncommon. Variations have been reported about branches of the brachial plexus and the distribution of the branches, but variations of posterior cord are partly rare. During routine dissection for undergraduate teaching, variations of the brachial plexus was found in the right upper extremity of a 72-year-old formalin-fixed female cadaver who had no pathological lesions, traumatic lesions or surgical procedures in the neck, thoracic or axillary region. In the right brachial plexus, it was observed that posterior cord had only two branches which were axillary and radial nerves. Upper subscapular nerve, thoracodorsal nerve and lower subscapular nerve emanated from axillary nerve. There was no other variations of brachial plexus has been observed in both sides. We thought it was quite rare variation that includes the upper subscapular nerve, the thoracodorsal nerve and the lower subscapular nerve are derived from axillary nerve we have discussed in this article. The knowledge of the lower subscapular nerve, the upper subscapular nerve and the thoracodorsal nerve emerge from the axillary nerve is mostly important for successful regional anesthesia, surgical interventions and radiological examination in this region.

Keywords: Posterior cord; subscapular nerves; thoracodorsal nerve; axillar nerve; variation.

\section{P-101}

\section{Three headed biceps brachii muscle: a case report}

Bilkay C, Candan B, Albay S

Departmant of Anatomy, Faculty of Medicine, Suleyman Demirel University, Isparta, Turkey

During our routine dissection studies for education, we encountered an accessory head of biceps brachii in the left upper extremity of a 73-year-old formalin-fixed male cadaver. The other two heads of the muscle and other arm muscles were as usual. Humeral accessory head of biceps brachii muscle have been classified according to the location of the head of biceps brachii muscle originate by some researchers as infero-medial humeral, superior humeral, infero-lateral humeral head. In this case, the accessory head originated from middle third of the humerus, superior and medial to the origin of brachialis muscle and inserted to the common tendon of biceps brachii. In the literature this head was named as infero-medial humeral head and its incidence changes between $7.7 \%-12 \%$ according to ethnicity. In our country, its incidence changes between $2.54 \%$ $-6.15 \%$. We think that knowledge of this type of the variation is important for prevention of possible surgical complications and diagnostic procedures.

Keywords: Biceps brachii muscle, variation, accessory head 


\section{P-102}

\section{Immunohistochemical changes in rat hypophysis related to the local beta-hCG injection}

Öktem $H^{*}$, Tunç $E^{* *}$, Çalgüner $E^{* * *}$, Erdoğan $D^{* * * *}$, Gözil R*** Bahçelioglu $M^{* * *}$, Göktaş $\mathrm{G}^{* * * *}$, Elmas Ç****

*Department of Anatomy, Faculty of Medicine, Baskent University, Ankara, Turkey; **Department of Anatomy, Faculty of Medicine, Maltepe University, Istanbul, Turkey; ${ }^{* *}$ Department of Anatomy, Faculty of Medicine, Gazi University, Ankara, Turkey; ${ }^{* * *}$ Department of Histology and Embryology, Faculty of Medicine, Gazi University, Ankara, Turkey

Objectives: Recently, it has been observed that weight loss is accelerated by human chorionic gonadotropin (hCG) hormone preparation used for hypothalamic dysfunction in obesity treatment in both sexes. Beta-hCG is also used for in vitro fertilization and in treatment of hypogonadotropic hypogonadism. Our aim was to observe the immunohistochemical changes caused by local injections of beta-hCG made for purpose of weight loss and to present them to inform those receiving such therapy.

Methods: In our study, 10 obese female, 10 male obese, 10 non-obese female and 10 non-obese male rats were used. In each group, single dose of subcutaneous beta-hCG injection has been applied to 7 rats for 5 weeks in 5 days of the week, and placebo has been applied to the remaining 3 rats. Following the injection, hypophysis were evaluated immunohistochemically.

Results: Progesterone receptor immunoreactivity was similar in all groups. The hypophysis tissue was seen that there are low retention in some cells within non-obese group having no betahCG implementation. In non-obese group having beta hCG implementation the reaction was seen in the settled cells under capsule. Within the obese group having no beta-hCG implementation, the reaction was higher than the previous group on the cells under capsule. In the obese group having beta-hCG implementation, it was seen that the reaction was lower in the tissue in comparison with the previous group. In conclusion the implementation of beta-hCG in addition to the diet has no effect on hypophysis.

Keywords: Beta-hCG, obesity, hypophysis, immunohistochemistry

\section{P-103}

\section{Unusual termination of the external jugular vein and cephalic vein}

Desdicioglu K, Erdoğan Öztürk K, Malas MA

Deparment of Anatomy, Faculty of Medicine, İmir Katip Çelebi University, Izmir, Turkey

During the routine cadaver dissection we performed in the dissection laboratory, it was observed that on the male adult cadaver aged 60 , the termination patterns of external jugular vein in the left neck region and cephalic vein in the upper left extremity were different. It was monitored that of external jugular vein in the left neck region of the cadaver joined with suprascapular vein from the left scapular region, that they constituted a common venous trunk and that this common venous trunk communicated with the venous angle. Separately, cephalic vein in the upper left extremity was determined to end in of external jugular vein in the neck region, instead of axillary vein. We are of the opinion that having the knowledge of these variations that we have described about external jugular vein and cephalic vein will be of use to the surgeons, radiologists and anesthetists involved in this region.

Keywords: Cadaver, vein, variation

\section{P-104}

\section{Cephalometric analysis in adult population by using computed tomography}

Erdoğan Öztürk K$K^{*}$, Tatlısumak E ${ }^{* *}$, Y1lmaz Ovalı G**, Pabuşçu $\mathrm{Y}^{* * * *}$, Tarhan $\mathrm{S}^{* * *}$

*Deparment of Anatomy, Faculty of Medicine, Izmir Katip Çelebi University, Izmir, Turkey; **Department of Anatomy, Faculty of Medicine, Celal Bayar University, Manisa, Turkey; Department of Radiology, Faculty of Medicine, Celal Bayar University, Manisa, Turkey

The aim of our study was to obtain normal craniofacial cephalometric measurements by using computed tomography (CT) in adult population, to investigate their relations with age and sex and with other measurements, and the evaluation of whether there was a difference between the measurements of right and left facial halves. CT images of 315 patients (159 male, 156 female) between the ages of 18-90 were used from the archive of radiology department of Celal Bayar University. The images of patients with fracture, abnormality and/or operated in the cranial region were excluded from the study. Totally 21 measurements, 6 of them bilateral, were obtained on the 3 different axial CT sections extending through cranial vault, orbita and zygomatic regions. The means of the measurements for total population and both sexes were calculated. Study group was separated into six groups according age and the changes of the measurements related to age were investigated. Relations between the measurements were evaluated with Pearson correlation analysis. Bilateral measurements of right and left facial halves were compared and the dominant side was determined. Statistical analysis were done with SPSS 15.0 program. $\mathrm{P}<0.05$ was accepted as significant. All measurements were larger in males and the differences of most of the measurements were statistically significant. It was determined that the midinterorbital and interzygomatic buttress distances were correlated with age. Bilateral measurements were usually larger at the right facial half. Asymmetry was not changing with sex and age. We suggest that data obtained from the normal individuals in the present study will be useful for the diagnosis and treatment of the craniofacial abnormalities and traumas. The presence of the correlations of midinterorbital distance and interzygomatic buttress distance with age shows that also bone structure changes have effects on facial aging. The presence of asymmetries in normal individuals is considered that a mild asymmetry occurs during facial procedures may be acceptable.

Keywords: Cephalometry, craniofacial measurements, facial asymmetry 


\section{P-105}

Evaluation of accessory canals in the anterior maxillary region by cone-beam CT: a preliminary study

Aydın $\ddot{U}^{*}$, Pelin $C^{* *}, \underline{\text { Külah K}}$

*Department of Dentomaxillofacial Radiology, Faculty of Dentistry, Baskent University, Ankara, Turkey; **Department of Anatomy, Faculty of Medicine, Baskent University, Ankara, Turkey

Objectives: Use of cone-beam computed tomography (CBCT) permits detailed investigation of the anatomical structures in the maxillofacial region. The present retrospective study aims to evaluate the accessory canals in the anterior region of maxilla by using CBCT.

Methods: CBCT scans of 30 patients, made either unilaterally or bilaterally, were included in the study. Of these 30 patients, 16 were females and 14 were males aged between 12-67 years. Anterior maxillary region including the distal aspects of the canines were evaluated in 49 sides. Scans in which the course of the accessory canals were not visible because of pathologic lesions or impacted teeth were excluded from the study. All the CBCT scans were evaluated by two observers in various planes by using the original software of the machine by which the scans were made. All the accessory canals with a diameter of at least $1.0 \mathrm{~mm}$ were evaluated regarding presence, location, course, as well as the age and gender of the patient. Frequency distribution test was used in statistical analysis.

Results: In six of the 49 sides, seven (14.3\%) accessory canals with a minimum diameter of $1.0 \mathrm{~mm}$ at the widest point were observed. Of these six patients, five were males and one was female and their ages were ranging from 20 to 55 years. The diameters of the accessory canals observed in CBCT scans were between 1.0 and 1.5 $\mathrm{mm}$. The foramina where the accessory canals terminated were located at the palatal side of the incisive teeth.

Conclusion: Accessory canals other than nasopalatine canal may be found in the anterior maxilla and of great importance for the surgical procedures in the region.

Keywords: Accessory canal, cone-beam computed tomography, maxilla

\section{P-106}

Evaluation of the hand antropometric measurement in ADHD children and the clinical significance of the 2D: 4D ratio in this population

Buru E*, Gozil R*, Iseri E**, Ozkan $\mathrm{S}^{* * *}$, Bahcelioglu $\mathrm{M}^{*}$

*Department of Anatomy, Faculty of Medicine, Gazi University, Ankara, Turkey; **Department of Child and Adolescent Psychiatry, Faculty of Medicine, Gazi University, Ankara, Turkey; ${ }^{* *}$ Department of Public Health, Faculty of Medicine, Gazi University, Ankara, Turkey

Attention deficit/hyperactivity disorder (ADHD) is characterized with decreased storage time of the information related to the lack of attention and behavioural changes such as hyperactivity and anxiety with hand features. Therefore; we compared individuals with ADHD and under treatment, measuring their hand and digit parameters especially 2D: 4D ratio with the healthy group.
Our purpose was to investigate the possibility to use the 2D: 4D ratio in clinics as an easy prediagnostic parameter. A total of 540 people (104 ADHD, 436 controls) between $7-17$ years old were included in our study. The case group was composed of 27 girls (26\%) and 77 boys (74\%) while the control group was constituted of 196 girls (45\%) and 240 boys (55\%). The hand longitudinal length, width, interval length, lengths of the first, second, third, fourth and fifth digits were measured from all participants right and left hands and 2D: 3D, 2D: 4D, 2D: 5D, 3D: 4D, 3D: 5D, 4D: 5D ratios were calculated. The delivery type and complications, chronic diseases and hand preferences were investigated in ADHD group. ADHD was more observed in $7-8$ years old group especially in boys. There was no statistically significant difference between the ADHD group and control group in 2D:4D ratio in both hands $(\mathrm{p}<0.05)$. Also, in ADHD group, no significant statistical changes were observed related to delivery type, complications and chronic diseases ( $p>0.05$ ). However, in ADHD boys, the 2D: 4D ratio was statistically significant in right handiness individuals. Our results pointed out that the ADHD and some other child psychiatric disorders could be early diagnosed related to the anthropologic parameters. So, the treatment of these individuals could be assigned in early period.

Keywords: Finger-length ratio, ADHD

\section{P-107}

Variations of brachioradialis muscle and superficial branch of radial nerve: a case report

Gozil R, Kastamoni Y, Bahcelioglu M

Department of Anatomy, Faculty of Medicine, Gazi University, Ankara, Turkey

The authors report a case of a male cadaver found to have two headed brachioradialis muscle tendon (BRMT) on both upper limb as revealed by routine dissection. The superficial branch of radial nerve (SBRN) descends in the forearm under the brachioradialis muscle (BRM) and then, pierced the deep forearm fascia between the BRMT and extensor carpi radialis longus tendon. In our case, we observed that BRMT was divided in two heads in both limbs and SBRN was passing between these two heads. Besides, on the right upper extremity, it was found that BRM with two heads.

Keywords: Brachioradialis muscle, tendon variation, superficial branch of the radial nerve, Wartenberg's syndrome

\section{P-108}

Beneficial effects of melatonin on arsenic-induced liver damage in rats

Uygur R*, Aktas $\mathrm{C}^{* *}$, Sener $\mathrm{U}^{* * *}$, Caglar $\mathrm{V}^{*}$, Yildirim $\mathrm{O}^{* * * *}$, Baltaci $\mathrm{BB}^{*}$, Uygur E*** Erboga $\mathrm{M}^{* *}$, Gurel A****, Ozen OA*

*Department of Anatomy, Faculty of Medicine, Namik Kemal University, Tekirdag, Turkey; **Department of Histology and Embryology, Faculty of Medicine, Namik Kemal University, Tekirdag, Turkey; ***, Department of Physiology, Faculty of Medicine, Tekirdag, Namik Kemal University Turkey; $\star * * \star$ Department of Gastroenterology, Faculty of Medicine, Namik Kemal University, Tekirdag, Turkey; $* * * *$ Department of Medical Biochemistry, Faculty of Medicine, Namik Kemal University, Tekirdag, Turkey 
The liver has long been identified as a target organ of arsenic exposure. Arsenic is a potent environmental toxicant and its accumulation in the liver causes hepatotoxicity. In our study, the investigation of effects of melatonin on arsenic-induced liver damage in rats was planned. In our study, 27 male rats were divided into three groups: control (saline, $5 \mathrm{ml} / \mathrm{kg} / \mathrm{day}$, intragastrically), arsenic (sodium arsenite, $5 \mathrm{mg} / \mathrm{kg} /$ day, intragastrically), and arsenic+melatonin (sodium arsenite, 5 $\mathrm{mg} / \mathrm{kg} /$ day, intragastrically and melatonin, $25 \mathrm{mg} / \mathrm{kg} /$ day, intraperitoneally) group. At the end of 15 days, the rats were weighed and sacrificed then liver tissues were collected. Superoxide dismutase (SOD), catalase (CAT), and glutathione peroxidise (GSH-Px) activities as well as malondialdehyde (MDA) levels were determined by spectrophotometry. The amount of arsenic in liver tissue was analyzed by Inductively Coupled Plasma/Optical Emission Spectrometer (ICP-OES). There were no significant differences in respect to weights of rats among the groups. Arsenic significantly decreased the enzyme activities of SOD, CAT, and GSH-Px as well as increased the levels of MDA in liver tissues. Melatonin treatments significantly increased of reduced SOD, CAT, and GSH-Px enzyme activities and decreased the elevated tissue MDA levels in liver tissues. The amount of arsenic in liver tissues significantly increased in arsenic group. There were no significant differences in respect to the amount of arsenic in liver tissues between the arsenic group and melatonin group. These data suggested that melatonin has beneficial effects against arsenic-induced liver damage by decreasing oxidative stress and lipid peroxidation.

Keywords: Arsenic, melatonin, liver, oxidative stress

\section{P-109}

Atypical localized giant-cell tumor of bone: case report Gul SS*, Uysal M**, Hasbek Z***, Bicakci H***, Tas U**

*Department of Nuclear Medicine, Faculty of Medicine, Gaziosmanpasa University, Tokat, Turkey; ${ }^{* *}$ Department of Anatomy, Faculty of Medicine, Gaziosmanpasa University, Tokat, Turkey; ${ }^{* *}$ Department of Nuclear Medicine, Faculty of Medicine, Cumhuriyet University, Sivas; $\star * * *$ Department of Anatomy, Faculty of Medicine, Sifa University, Izmir, Turkey

Giant-cell tumor of bone (GCT) composed of undifferentiated cells of a neoplastic alarm that is fusion of a large number of multinucleated giant cells and stromal cells or proliferated mononuclear cells. It usually seen at the distal femur, proksimal tibia and distal radius. This neoplasm typically seen between the ages of 25-35 and it is rare to see under age of 20 and over the age of 50. Although computed tomography is useful for determining the extent of the lesion, it does not provide much advantage on the standard radiography about the tumor's characteristics and aggressiveness. In this paper we presented an atypical area located GCT of bone at the proksimal right femur, by a three-phase whole-body bone scintigraphy.

Case report: At the bloody and blood pool images of right femur proximal third portion, area increasing of perfusion and hyperemia, at the late-phase static images of the right femur proximal diaphyseal region, an increased heterogeneous osteoblastic activity expansile characterized involvement was falowed at the three-phase bone scintigraphy of a female patient with right leg pain. The patient was diagnosed as GCT with clinical and radiological assessment. DHT is encountered more frequently in women as compared to men. In accordance with literature in our case, GCT was seen at a female patient. However, in contrast to the literature data, the localization of tumor was at an atypical place in our case. It should be kept in mind that the lesion can localizated in atypically place as in this case, though GCT usually places at distal femur, proximal tibia and distal radius.

Keywords: Giant-cell tumor of bone, whole body bone scintigraphy, technetium-99m

\section{P-110}

\section{Protective effects of cape in carbon tetrachloride- induced liver damage in Wistar albino rats}

Cetin A, Elbe H, Taslidere E, Gul M, Otlu A

Department of Histology and Embryology, Faculty of Medicine, Inonu University, Malatya, Turkey

Objectives: Carbon tetrachloride $(\mathrm{CCl} 4)$ is a substance, led to liver damage with caused to free radical formation. These free radicals creates lipid peroxidation and membrane damage occurs with toxic lipid peroxidation products. If this does not prevent membrane damage, cell death occurs. Caffeic acid phenyl ester (CAPE), a flavonoid- like compound and an active component of propolis from honey bee hives. CAPE has strong, immunomodulatory and antioxidant properties. In this study, we planned to determine the protective effects of CAPE on liver damage induced by $\mathrm{CCl} 4$.

Methods: In this study, 28 rats were randomly divided into 4 groups. Groups: Group 1: Control (5\% ethanol, $1 \mathrm{ml} / \mathrm{day} / \mathrm{ip})$, Group 2: Olive oil (0.5 ml/every other day/ip), Group 3: $\mathrm{CCl}_{4}$ $\left(0.5 \mathrm{ml} / \mathrm{kg}\right.$ /every other day/ip), Group 4: $\mathrm{CCl}_{4+\mathrm{CAPE}}(0.5$ $\mathrm{ml} / \mathrm{kg} /$ every other day/ip $+10 \mu \mathrm{mol} / \mathrm{kg} / \mathrm{day} / \mathrm{ip})$. The tissue samples take in $10 \%$ formaldehyde, processed by routine tissue techniques and embedded in paraffin. 5 - $\mu \mathrm{m}$ thick sections of tissues were cut, mounted on slides, stained with HematoxylinEosin (H-E) and exam-ined under Leica DFC280 light microscope, Leica Q Win and Image Analysis System.

Results: In control and olive oil groups, liver showed normal histological appearance. In $\mathrm{CCl}_{4}$ group, distortion of radial arrangement of hepatocytes, eosinophilic stained and pyknotic nuclei cells, mononuclear cell infiltration, hepatocyte vacuolization, vascular congestion and hemorrhage were observed. Histopathological changes in $\mathrm{CCl}_{4}+\mathrm{CAPE}$ group were not as common in $\mathrm{CCl}_{4}$ group.

Conclusion: In conclusion, the results of this study suggest that CCl4-related liver toxicity in rats could be significantly reduced by CAPE administration.

Keywords: $\mathrm{CCl}_{4}$, CAPE, liver, hepatotoxicity. 


\section{P-111}

Anaglyph form of the ventricular system of the brain in the rat

Terim Kapakin $\mathrm{KA}^{*}$, Kapakin $\mathrm{S}^{* *}$

*Atatürk University Faculty of Veterinary Medicine Department of Pathology, Erzurum, Turkey; **Department of Anatomy, Faculty of Medicine, Ataturk University, Erzurum, Turkey

The aim of this study was to reconstruct the ventricular system of the rat brain three-dimensionally and generate anaglyph form of it. In our case, left and right colour stereo pairs of images of the ventricular system of the rat brain were put together to effectuate anaglyphs by Adobe Photoshop. First, both images were converted to greyscale images, and they were then converted back to RGB (red, green, blue) images. Subsequently, the red component of the left stereo pair was replicated to the red component of the right stereo pair, eliminating the red component of the right image. A duo-chromatic digital image was generated and that could be printed as a photorealistic image by using photographic-quality printers. It can be possible to follow up the changes in the shape and size of the ventricular system of the rat brain. Clinicians can usually detect disease with the change in the shape and size of the ventricular system of the rat brain.

Keyword: Rat, ventricular system of the rat brain, anaglyph

\section{P-112}

Formaldehyde inhibition of irisin production in blood, liver and lung tissues of rats: direct evidences that carnosine alleviate the formaldehyde inhibition of irisin

$\underline{\text { Aydin } S^{*}, * *}$, Ogeturk $M^{*}$, Kuloglu T ${ }^{* * *}$, Kavakli A*, Aydin $S^{* * * *}$ *Deparment of Anatomy, Faculty of Medicine, Firat University, Elazig, Turkey; **Clinic of Cardiovascular Surgery, Elazig Research and Training Hospital, Elazig, Turkey; ***Deparment of Histology and Embryology, Faculty of Medicine, Firat University, Elazig, Turkey; $\star * * *$ Deparment of Medical Biochemistry (Firat Hormones Research Group), Faculty of Medicine, Firat University, Elazig, Turkey

Formaldehyde (FA) is one of the most common organic compounds used in today's world. This commonly used organic compound is dangerous to life and health at 20 ppm by disrupting mitochondrial energy metabolism and possibly irisin production, which has an important role in energy metabolism. To alleviate the possible defects of irisin production and mitochondrial energy metabolism, carnosine might play an important role by combating the effects of free radical. In this study, we provide evidence supporting this assumption. FA (which is known to disrupt mitochondria) was tested over a range of concentrations regarding effects on irisin production, terminal deoxynucleotidyl transferase-mediated deoxy-uridine-triphosphate (dUTP) nick end labeling ((TUNEL), a common method for detecting DNA fragmentation, in blood, liver and lungs tissues of rats in the range of $0.0-15 \mathrm{ppm}$ FA and at 100 $\mathrm{mg} / \mathrm{kg}$ carnosine. Above $5 \mathrm{ppm}$ FA concentrations inhibit irisin production in tissues and serum while having a much higher effect on lung tissues. However, $100 \mathrm{mg} / \mathrm{kg}$ carnosine supplementation reversed the inhibition of irisin production of liver and lungs seen at 5-15 ppm FA, linking the presence of carnosine with the increase in irisin production in serum and tissues. We concluded that carnosine supplementations alleviate the hazardous effects of FA in biological tissues.

Keywords: Formaldehyde, irisin, liver and lung tissues

\section{P-113}

A case of the sural nerve originated from the common fibular nerve

Erdoğan Ozturk K, Çizmeci G, Desdicioğlu K, Malas MA

Department of Anatomy, Faculty of Medicine, Izmir Katip Celebi University, Izmir, Turkey

The sural nerve is formed by merging of medial sural cutaneous nerve and lateral sural cutaneous nerve nearby the middle of posterior of the leg, which arise from the tibial nerve and common fibular nerve respectively,. Then, it descends with small saphenous vein towards to lateral malleolus and extends at the lateral side of the dorsum of the foot to the little toe as lateral dorsal cutaneous nerve. Variations of sural nerve are quite important for the surgeons who work in this region due to frequent usage as biopsy and nerve transmission rate in diagnosis and nerve graft in therapy. We observed during the routine cadaver dissections in our department laboratory that sural nerve in the lower-left extremity of an adult male cadaver has stemmed from common fibular nerve. We also observed that sural nerve traced towards to distal part of the leg on the lateral side of the small saphenous vein. The course of sural nerve in the lower-right extremity was determined as normal. Various studies were determined about sural nerve in the literature. We believe that the variation we defined in this study be beneficial for the radiological and neurological studies as well as surgical operations related to that area.

Keywords: Sural nerve, cadaver, variation

\section{P-114}

Anatomy and variations of plantaris muscle in fetuses

Desdicioglu K*, Uguz C ${ }^{* *}$, Sakallı B***, Koyuncu E ${ }^{* * *}$, Malas MA* $^{*}$

*Department of Anatomy, Faculty of Medicine, Izmir Katip Celebi University, Izmir, Turkey; **Department of Anatomy, Faculty of Medicine, Mugla Sıtkı Kocman University, Mugla, Turkey, ***Department of Anatomy, Faculty of Medicine, Suleyman Demirel University, Isparta, Turkey

Objectives: We have aimed at researching into the morphometric development and variations of the plantaris muscle and its tendon throughout the fetal period and comparing them with the the results of the study performed on adults.

Methods: A total of 102 fetal legs (51 human fetuses: 26 male, 25 female) whose ages varied between 15-40 gestational weeks without any external pathology or anomaly were incorporated in the study. The fetuses were divided into groups according to 
gestational weeks, trimesters and months. After the general external measurements of the fetuses had been made, the leg dissection was performed. Later on, the morphometric parameters of plantaris muscle were measured.

Results: The averages and standard deviations of the measured parameters were determined according to gestational weeks, trimesters and months. There was a significant correlation between the measured parameters and the gestational age $(\mathrm{p}<0.001)$. There was no difference between genders in terms of parameters $(\mathrm{p}>0.005)$. All the obtained results were discussed by making comparisons with the previous studies.

Conclusion: We are of the opinion that the data obtained in our study will be of use to the involved clinicians in the evaluation of the development of plantaris muscle, and in the clinical studies and practices.

Keywords: Fetal development, morphometry, plantaris muscle, anatomy

\section{P-115}

\section{A variation of the thyroid cartilage:} absence of left superior cornua

Kosar $\mathrm{MI}^{*}$, Tetiker $\mathrm{H}^{*}$, Uguz Gencer $\mathrm{C}^{*}$, Balci $\mathrm{Y}^{* *}$, Sahan $\mathrm{M}^{\star * *}$

*Department of Anatomy, Faculty of Medicine, Mugla Sıtkı Kocman University, Mugla/ Turkey; ${ }^{* *}$ Department of Forensic Medicine, Faculty of Medicine, Mugla Sitkı Kocman University, Mugla, Turkey; ***Department of Otolaryngology, Faculty of Medicine, Mugla Sitkı Kocman University, Mugla, Turkey

The thyroid cartilage is the largest of the laryngeal cartilages, and forms mainly the anterior and lateral wall of the larynx. Thyroid cartilage have two superior and two inferior cornua. Superior cornua may be absent congenitally or dislocations may be caused by trauma. The present case is reported to emphasis a rare anatomic variation of the thyroid cartilage. A 70-year old male, at the autopsy performed by a forensic expert was found died from cardiovascular disease. In the autopsy, during the throat examination, hyoid bone and thyroid cartilage was found intact, but left horn of the thyroid cartilage was absent at the anatomical place. This condition was seemed to be an anatomical variation. In this case, we were suggested that the absence of superior cornua was noticed on the left side. Sometimes, there is a complete agenesis of one or several cornua. These anatomical variations have been described in the literature, rarely. The knowledge of this anatomical variation would be useful to interpret different clinical condition.

Keywords: Cornu superior, cartilago thyroidea, laryngeal anomalies, forensic autopsy, congenital defects

\section{P-116}

A morphometric study on proximal femur in dried bones

Akin D, Aydin Kabakçi AD, Yilmaz MT, Seker M, Korkut Z Department of Anatomy, Meram Medicine Faculty, Necmettin Erbakan University, Konya, Turkey
Objectives: The femur is the strongest and the longest bone in the body. Generally, it is up ? femur lenght. The purpose of this study is morphometric analysis of proximal femur in dried bones and comparing with studies in the literature.

Methods: This study was conducted on 38 femur (21 right, 17 left) in collections of Necmettin Erbakan University Meram Medicine Faculty Anatomy Labratory. Digital calipers, osteometric board and precision scales were used for femoral bone measurements. Only proximal femur bone measuremens were performed. It was identified whether or not a relationship between femur length and femur weight. Each bone weight was determined with the help of precision scales. Also nutrician foramen number and localization was determined.

Results: In this study, it was determined that mean right maximum femur lenght (RMFL) 42,57 $\pm 2,64 \mathrm{~mm}$, mean left maximum femur length $42.34 \pm 2.04 \mathrm{~mm}$. It was identified that mean right femur weight was $328.612 \pm 72.38 \mathrm{gr}$, mean left femur weight was $292.90 \pm 63.72$ gr. Furthermore, it was found that mean right femur vertical diamater (RFVD) $41.66 \pm 4.02 \mathrm{~mm}$ and transverse diamater (RFTD) $44.21 \pm 3.91 \mathrm{~mm}$, mean left femur vertical diameter (LFVD) $40.34 \pm 2.98 \mathrm{~mm}$ and transverse diamater (LFTD) $41.97 \pm 3.44 \mathrm{~mm}$. It has been determined that high correlation rate between maximum femur lenght and maximum femur wieght on both sides.

Conclusion: Morphometric measurements of proximal femur were performed in this study. We believe that the obtained datas will be helpful for construction of femoral prothesis.

Keywords: Femur, morphometry, anatomy

\section{P-117}

\section{Investigation of dose-dependent effects of mianserin on diabetic nephropathy model}

\section{Alpay M*, Can DO**, Demir Ozkay U**, Yucel F*}

*Department of Anatomy, Faculty of Medicine, Eskisehir Osmangazi University, Eskisehir, Turkey; **Department of Pharmacology, Faculty of Pharmacy, Anadolu University, Eskisehir, Turkey

Mianserin is a tetracyclic antidepressant drug which has noradrenergic and serotonergic effects. According to international treatment guidelines, it is one of the first preferred drug in the treatment of depression patients having low renal index. However, there is no morphometric study investigating the effects of Mianserin on kidneys. The purpose of this study was to investigate the effects of Mianserin on the kidneys of the diabetic rats. Male Wistar rats were divided into six groups: (1) Healthy controls (HC), (2) healthy rats with the daily application of $30 \mathrm{mg} / \mathrm{kg}(\mathrm{H}+30 \mathrm{mg} / \mathrm{kg})$ and (3) $45 \mathrm{mg} / \mathrm{kg}$ Mianserin $(\mathrm{H}+45 \mathrm{mg} / \mathrm{kg})$, per oral (4) diabetic rats (DM) by injection of a single dose of streptozotocin $(50 \mathrm{mg} / \mathrm{kg})(5)$ diabetic rats with the daily application of $30 \mathrm{mg} / \mathrm{kg}(\mathrm{DM}+30 \mathrm{mg} / \mathrm{kg})$ and (6) 45 $\mathrm{mg} / \mathrm{kg}$ Mianserin (DM+45 mg/kg). After two-week application of Mianserin, rats were perfused cardiacally and then 4 İmthickness sections taken by a systematic random sampling method from each kidney stained with Hemotoxylin-Eosine. Alterations occurring in the kidneys were estimated by using 
morphometric methods. Results were compared with one-way analysis of variance test following Bonferroni multiple comparison tests. In the DM group, ratio of the Bowman's space to renal corpuscule was found significantly higher $(23.93 \pm 8.91)$ than other groups. This ratio was decreased in diabetic groups treated with Mianserin in a dose dependent fashion (12.05 \pm 7.32 and $9.92 \pm 3.30$ ). However, in healthy groups, no significant difference was observed following Mianserin treatment. On the other hand, number of renal corpuscule per unit renal cortical area $(\mathrm{Na})$ and the mean diameter of the renal corpuscule were not significantly different among groups. These results show that Bowman's space expands with regard to the diabetes and Mianserin treatment reverses these diabetic alterations.

Keywords: Mianserin, kidney, diabetes mellitus

\section{P-118}

\section{Investigation of the upregulation of VEGF in mice retina after retinal laser photocoagulation using argon and pattern scan laser system}

Konac E*, Sonmez $\mathrm{K}^{* *}$, Bahcelioglu $\mathrm{M}^{* * *}$, Take Kaplanoglu $\mathrm{G}^{* * * *}$, Varol N*, Sarac GN**** Ozcan PY**

*Department of Medical Biology and Genetics, Faculty of Medicine, Gazi University, Ankara, Turkey; **Ulucanlar Eye Research and Education Hospital, Ankara, Turkey; ***Department of Anatomy, Faculty of Medicine, Gazi University, Ankara, Turkey; $* * * *$ Department of Histology and Embryology, Faculty of Medicine, Gazi University, Ankara, Turkey

Panretinal photocoagulation still remains the gold standard for the treatment of ischaemic and neovascular diseases of the retina. Pulse energy is directly proportional to the pulse duration and power. Vascular endothelial growth factor (VEGF) is a well-known potent angiogenic factor and also acts as a proinflammatory cytokine and vascular permeability factor. The aim of this study was to investigate the differences in the expression of VEGF in mice subjects treated with argon (AG) or pattern scan laser (PASCAL) systems. Male C57BL/6 mice were randomly assigned to one of three groups: Group 1 (G1) AG photocoagulator $(\mathrm{n}=16)$, Group 2 (G2) PASCAL $(\mathrm{n}=16)$ and Group 3 (G3) control group $(n=6)$. Molecular and morphological analyses of VEGF were performed on day 1, 2 and 5 by ELISA, Real-time PCR and immuno-histochemical analysis. G1 group showed VEGF mRNA level increased 2.4 folds on day 2, whereas it decreased on day $5(\mathrm{p}<0.001)$ compared to the $\mathrm{G} 3$. In G2, VEGF mRNA level increased 1.8 folds on day 1 and 2.2 folds on day 5 compared to the G3. In G1, VEGF protein level increased significantly on day 2, whereas it decreased on day 5 ( $\mathrm{p}<0.001$ ) compared to G3. In group G2, the VEGF levels significantly increased as compared to control groups at both 2 and 5 days after laser photocoagulation using PASCAL laser ( $\mathrm{p}<0.001$, both time points). The peak expressions of VEGF protein in samples which underwent PASCAL and conventional laser were found on day 5 and day 2 respectively. In PASCAL photocoagulation pattern, VEGF-induced vascularization started late and gradually increased; in AG, VEGF- induced vascularization started early on day 2, then decreased on day 5 .

Conclusion: AG laser may imposed a higher risk for early complications due to early increase in VEGF levels, whereas the complications due to increment in VEGF level is not expected to be different between conventional and PASCAL laser treatment in the late phase.

Keywords: Conventional Laser, Pattern Scan Laser (PASCAL), VEGF, gene expression, angiogenesis

\section{P-119}

Osgood-Schlatter disease: a case report

Sagıroglu A*, Eldeleklioglu $\mathrm{S}^{* *}$, Acer $\mathrm{N}^{* * *}$

*Department of Anatomy, Kayseri Education and Research Hospital, Kayseri, Turkey; ${ }^{* *}$ Kayseri Education and Research Hospital, Children's Health and Diseases, Kayseri, Turkey; ${ }^{* *}$ Department of Anatomy, Faculty of Medicine, Erciyes University, Kayseri, Turkey

Objectives: Osgood-Schlatter disease (OSD) is a common cause of pain and tenderness at the tibial tuberosity in active adolescents who play sports. It is more common in boys. The common age for boys to have OSD between 12 and 15 years, and for girls, between 8 and 12 years. The etiology of OSD is not known exactly. There are some etiologic theories, such as degeneration of the patellar ligament, aseptic necrosis, systemic disease, endocrinopathy and infection. 20-30\% of cases are affected in both knees. We consider that our case may contribute to diagnose for similar studies to be conducted.

Results: We reported of a 13 -year-old boy who presented to Kayseri Education and Research Hospital of emergency department with a 1-week history of progressively worsening right knee pain. There was no limited range of motion or overlying erythema on the skin. The $\mathrm{x}$-ray radiography supported the OSD diagnosis.

Conclusion: Radiographic studies are helpful in diagnosis and treatment of OSD and similar pathologies.

Keywords: Osgood-schlatter disease, tibial tuberosity, knee pain, adolescent

\section{P-120}

\section{Immunohistochemical evaluation of potential changes in the hippocampus of rats fed with whey protein support}

\section{Tuc Yucel A*, Gurgen AG**, Kocturk S***}

*Department of Anatomy, Vocational Health Service, Celal Bayar University, Manisa, Turkey; ${ }^{*}$ Celal Bayar University Vocational Health Service, Department of Histology and Embryology, Manisa, Turkey; $* * *$ Department of Clinical Biochemistry, Faculty of Medicine, Dokuz Eylul University, Izmir, Turkey

The uninformed use of whey protein support among young people and amateur sportspeople has increased considerably. The aim of this study was to compare the effects of whey protein on 
brain tissue in unexercised rats in the long and short terms. Thirty male Wistar albino rats were divided into three groups; a control group, a group receiving short-term whey protein (5 days, $252 \mathrm{~g} / \mathrm{kg}$ ) (WS) and a third group receiving long-term whey protein (4 weeks, $252 \mathrm{~g} / \mathrm{kg}$ ) (WL). Neurofilament (NF), glial fibrillary acidic protein (GFAB), brain-derived neurotrophic factor (BDNF) and N-methyl-D-aspartate receptor (NMDAR) signaling molecules in the hippocampal region in dissected brain tissues were assessed using immunohistochemistry. GFAB and NF reaction in the hippocampal region was moderate in all three groups. There was no significant difference in terms of expressions. At BDNF immunostaining, reactions were weak and similar in all three groups. NMDAR expression in the control group hippocampus was moderate, while there was a very minor decrease in the short- and longterm groups. These findings show that the use of whey protein causes no changes in the release of NF, GFAB and BDNF neural factors in the hippocampal region of the brain. This in turn indicates that no neural transmission or neuroglial cell life problems occur. However, whey protein did cause a very minor decrease in NMDAR signaling molecule expression in the hippocampus compared to the control group. This suggested that sportspeople using whey protein may experience memory problems, albeit minor ones.

Keywords: Whey protein, brain, NF, GFAB, BDNF, NMDAR

\section{P-121}

\section{Investigation of the effect of whey protein on signaling molecules and testosterone release in the seminiferous tubules in the testis}

Gurgen $S^{*}$, Tuc Yucel A** , Kocturk $\mathrm{S}^{* * *}$

*Department of Histology and Embryology, Vocational Health Service, Celal Bayar University, Manisa, Turkey; ${ }^{*}$ Department of Anatomy, Vocational Health Service, Celal Bayar University Manisa, Turkey; $* * *$ Department of Clinical Biochemistry, Faculty of Medicine, Dokuz Eylul University, Izmir, Turkey

It is unclear whether whey protein, popular with many sportspeople due to its high calorific value and because it increases body mass, has adverse effects on the male reproductive system. The aim of this study was to investigate the effects of whey protein on apoptotic signaling molecules and testosterone release in the testis. Thirty male Wistar albino rats were divided into three groups, a control group, a group receiving short-term whey protein (5 days, $252 \mathrm{~g} / \mathrm{kg}$ ) (WS) and a group receiving long-term whey protein (4 weeks, $252 \mathrm{~g} / \mathrm{kg}$ ) (WL). Immunohistochemical techniques were applied to P-53, Bax, Bcl-2 and cytochrome-c signaling molecules and testosterone in extracted testis tissue. In the control group, seminiferous tubules P53 reaction was observed in some primary spermatocytes (PSs) and Sertoli cells (SCs), while in the WS group, expression ranging from moderate to strong was determined in most PSs and SCs. In the WL group, strong immune reaction was observed in both PSs and SCs. No Bax immunoreaction was encountered in any group. Bcl-2 expression was moderate in control group PSs but weaker in the other two groups.
Cytochrome-c expression in the control group was weak and granular in form in secondary spermatocytes (SSs), while expression in the WS and WL groups was again observed only in SSs and in strongly granular form. Testosterone immunoreaction exhibited strong release in control group Leydig cells, while expression was moderate in WS and WL. Whey protein use, and particularly long-term use, causes damage via the P-53 signaling molecule in SSs, which are of vital importance for sperm nourishment and support in the seminiferous tubules. It also causes excessive expression of the cytochrome-c molecule in SSs through the mitochondrial pathway. Both long- and short-term use can lead to some degree of decrease in testosterone release from Leydig cells.

Keywords: Whey protein, testis, P-53, Bax, Bcl-2, cytochrome-c, testosterone

\section{P-122}

\section{An examination of the effect of metoclopramide on proliferation signal molecules in newborn liver tissue}

Tuc Yucel A*, Gurgen SG**, Umur N***, Gozukara $\mathrm{C}^{* * * *}$, Kabaroglu $\mathrm{C}^{* * * *}$, Onur $\mathrm{E}^{* * * *}$

*Department of Anatomy, Vocational Health Service, Celal Bayar University, Manisa, Turkey; **Department of Histology and Embryology, Vocational Health Service, Celal Bayar University, Manisa, Turkey; ${ }^{* *}$ Department of Biochemistry, Vocational Health Service, Celal Bayar University, Manisa, Turkey; ${ }^{* * *}$ Department of Medical Biochemistry, Faculty of Medicine, Celal Bayar University, Manisa, Turkey; $* * * * *$ Department of Clinical Biochemistry, Faculty of Medicine, Ege University, Izmir, Turkey

Metoclopramide is an anti-emetic drug with hyperprolactinemic effects widely used in nausea and vomiting, and recently in the lactation period. There are no studies at the molecular level in the literature concerning potential side-effects in the newborn caused by the use of metoclopramide during breastfeeding. The purpose of this study was to investigate the potential proliferative changes in newborn liver tissue caused by metoclopramide. 18 young adult female Wistar albino rats that had recently given birth, together with their pups, were divided into 3 groups; Group 1: healthy control. Group 2: administered a low dose of metoclopramide $(10 \mathrm{mg} / \mathrm{kg}$ for 7 days, twice daily, ip), and Group 3: administered a high dose of metoclopramide (50 mg/kg 21 days, twice daily, ip). The experiment continued throughout the lactation period. Pups' hepatic tissues were removed under light microscopy at the end of the 21st day. 5bromo-2-deoxyuridine (BrdU), Ki-67 and proliferating cell nuclear antigen (PCNA) were used in immunohistochemical staining. Control group BrdU and PCNA immunoreactions in hepatocytes around the central vein were of moderate intensity and nuclear. Numbers of BrdU and PCNA positive cells decreased in hepatocytes in the low-dose group, and particularly in the high-dose group. At Ki-67 immunostaining, in complete contrast, cells showing reaction around the central vein were few in number in the control group, while there was a marked increase in numbers in the low- and high-dose groups. PCNA, used to show rate of proliferation in the cell, is 
a DNA replication protein synthesized in the late $\mathrm{G} 1$ and S phases of the cycle. BrdU is only used to show cells in the $S$ phase. The decrease in cells in the $S$ phase in livers in both the low- and high-dose metoclopramide groups shows that this agent compromises DNA synthesis in the cell. Higher Ki-67 in the experimental groups compared to the control group suggests that it causes an excessive proliferation index.

Keywords: Metoclopramid, Liver, BrdU, Ki-67, PCNA

\section{P-123}

The relationship of hand dominance and dominant eye to some diseases of university students

Aliosmanoglu B*, Kockar C**, Unal KS ${ }^{* *}$, Aliosmanoglu A***

*Department of Anatomy, Institute of Health Sciences, Gaziantep University Gaziantep, Turkey; ${ }^{*}$ Department of Nursing, Health High School, Hasan Kalyoncu University, Gaziantep, Turkey; ***Doğal Yaşam Special Education and Rehabilitation Center, Gaziantep, Turkey

Objectives: Cerebral lateralization is different abilities that are shown during specific neurological functions of the cerebral hemispheres in the acquisition and control. Cerebral dominance refers to the dominance of one of the brain hemispheres. There are studies about right hemisphere dominance in the direction of slowing the development of the body's immune mechanisms. In these studies, the clinical condition of the subject associated with cerebral the lateralization, in some patient subgroups left hand dominance and increased rates are seen to be two handedness. In our study, we had investigated the hand preference and eye dominance relationships with some of the diseases.

Methods: 95 students were applied exactly Old Field Preference Survey, Hand Preference Questionnaire, Beck Depression Inventory, dominant eye test. Subjects were asked if they had the diagnosis of any cardiovascular, respiratory system, digestive system, urogenital system, nervous system, musculoskeletal system, endocrine system diseases or rheumatic diseases, allergy, diabetes, vision, hearing and speech problems.

Results and Conclusions: Left-handedness rate is $21.2 \%$, right-handedness is $71.4 \%$, and both handedness rates is $7.4 \%$. $50.5 \%$ of the participants have the dominant eye as left. $21.5 \%$ of the right handed have respiratory disease, $20.0 \%$ allergy in the storyline, $1.5 \%$ rheumatic disease, $9.2 \%$ nervous system disease, $23.1 \%$ the vision problems. For left handed, $20.0 \%$ have the respiratory system disease, $10 \%$ allergy history, $20.0 \%$ the nerve system disease, $20.0 \%$ visual impairment, $1.5 \%$ rheumatic disease were observed. Compared with hand preference and rheumatic diseases, the two-handeds were observed in favor with $p$ ? 0005 level $(p=0.004)$. When compared with hand preference and to be diagnosed with the disease, highly significant ratio was observed in favor of right handed patients $(\mathrm{p}<0.001, \mathrm{p}=0.000)$.

Keywords: Cerebral lateralization, hand dominance, dominant eye, immunity

\section{P-124}

The evaluation of the protective effect of lumbricus extract for brain and the effect on antioxidant system in unilateral common carotid artery ischemia/reperfusion model

Canbaz Kabay S*, Öz S**, Özden $\mathrm{H}^{* * *}$, Burukoğlu D****, Kuş $\mathrm{G}^{* * * * *}, \underline{\text { Yeğin } \mathrm{B}^{* * *}}$, Üstüner $\mathrm{CM}^{* * * * *}$, Şentürk $\mathrm{H}^{* * * * * *}$, Misırlıoğlu $M^{* * * * * *}$, Y1ldız $F^{* * * * * * *}$, Aydemir D***

*Department of Neurology, Faculty of Medicine, Dumlupınar University, Kutahya, Turkey; **Vocational School of Health Services, Eskisehir Osmangazi University, Eskisehir, Turkey; ${ }^{* *}$ Department of Anatomy, Faculty of Medicine, Eskisehir Osmangazi University, Eskisehir, Turkey; $* \star * *$ Department of Histology and Embryology, Faculty of Medicine, Eskisehir Osmangazi University, Eskisehir, Turkey; $* * * * *$ Open Education Faculty, Anadolu University, Eskisehir, Turkey; ${ }^{* *}$ Department of Anatomy, Faculty of Medicine, Eskisehir Osmangazi University, Eskisehir, Turkey; $* * * * * *$ Department of Medical Biology, Faculty of Medicine, Eskisehir Osmangazi University, Eskisehir, Turkey; $* * * * * \star *$ Department of Biology, Faculty of Science, Eskisehir Osmangazi University, Eskisehir, Turkey; $* * * * * * \star$ Department of Biology, Faculty of Science, Eskisehir Osmangazi University, Eskisehir, Turkey; $* * * * * * *$ Department of Medical Laboratory Techniques, Toros University, Mersin, Turkey;

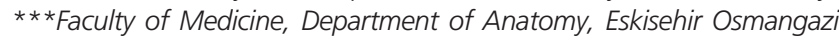
University, Eskisehir, Turkey

Objectives: The main purpose of the study is to research the protective effect of the Lumbricus Extract (LE) for prevention from the damage of the brain in the common carotid artery ischemia/reperfusion model.

Methods: In this study, 50 Spraque Dawley male rats were used. They divided in to 5 groups. Group-A was the control group which was applied only dissection of the left common carotid artery. Group-B was the ischemia group which dissected and clemped left common carotid artery. Group-C was the group which applied reperfusion 24 hour later after 15 minute left common carotid artery ischemia application following 20 $\mathrm{mg} 14$ day / single $2 \mathrm{ml}$ dosage of LE extract by gavage. Group$\mathrm{D}$ was the group which applied reperfusion 24 hour later after 15 minute left common carotid artery ischemia application following $40 \mathrm{mg} 14$ day / single $2 \mathrm{ml}$ dosage of LE extract by gavage. Finally, Group-E was the group which applied reperfusion 24 hour later after 15 minute left common carotid artery ischemia application followed $80 \mathrm{mg} 14$ day / single $2 \mathrm{ml}$ dosage of LE extract by gavage. The malondialdehyde (MDA) and antioxidant enzyme levels of the subjects were measured. Histologic evaluation was made after hematoxylin and eosin coloring.

Results: Histopathologic findings: For the Control Group (Group A) it was observed that there were normal like neurons and glial cells in the cortical area. Intensive damage and necrotic neurons were observed in the cortical area. However, the normal looking neurons together with very few necrotic neurons in the cortical area were observed in the $20 \mathrm{mg} / \mathrm{kg} \mathrm{LE}$ group. It was also observed the damage in the cortical area continued. On the other hand, normal-like neurons and glial cells as well as decreased damage in the cortical area were observed for the $40 \mathrm{mg} / \mathrm{kg} \mathrm{LE}$ group. In $80 \mathrm{mg} / \mathrm{kg}$ LE group it was seen 
that cortical damage were decreased and there were nearly normal neurons and glial cells. The MDA levels were increased in the ischemia and ischemia/reperfusion groups while this increase was prevented in the treatment groups. Similarly, Catalaz (CAT) and Super Oxide Dismutase (SOD) levels were decreased in the ischemia and ischemia/reperfusion groups while this decrease was prevented in the treatment groups.

Conclusion: Lumbricus extract may have an anti-thrombotic and anti-apoptotic impact. There are also studies that exhibit they are stimulating the nerve regeneration. In our study, we observed that LE has a protective effect against the brain damage created by left common carotid artery ischemia/reperfusion.

Keywords: Lumbricus, ischemia/reperfusion injury, antioxidant, brain

\section{P-125}

Tendinous slips crossing between flexor hallucis longus and flexor digitorum longus tendons in the sole of the foot

Uzel $M^{*}$, Gumusalan $\mathrm{Y}^{* *}$, Tugtag B** Cetınus $\mathrm{E}^{* * *}$

*Department of Orthopaedics and Traumatology, Faculty of Medicine, Kahramanmaraş Sütçü Imam University, Kahramanmaras, Turkey; ${ }^{* *}$ Department of Anatomy, Faculty of Medicine, Fatih University, Istanbul, Turkey; ${ }^{* *}$ Clinic of Orthopaedics and Traumatology, Ministry of Health, Haseki Research and Training Hospital, Istanbul, Turkey

Objectives: Flexor hallucis longus (FHL) and flexor digitorum longus (FDL) muscles are long flexor muscles of the toes. These muscles provide a wide and stable supporting area on which the body will bear forward while the foot was in contact with the ground. FHL and FDL muscles flex the toes especially distal interphalangeal joints when there is no contact of the foot with the ground. They work simultaneously together with the intrinsic muscles in order to sustain close contact of digital pillows with the ground when the foot is on the ground and carrying a burden. By this means, weight bearing area is widened and metatarsal heads are stabilized in order to use as a lever on which the body will be oriented forward. It is necessary to increase the awareness of clinicians about the structural changes of tendons and intertendinous crossings especially for the accurate diagnosis and treatment of foot injuries as well as in tendon transfer procedures.

Methods: Twenty-five lower extremities belonging to adults without having congenital structural anomalies which were amputated due to ischemic reasons were preserved in deep freezer at $-18^{\circ} \mathrm{C}$. After loosening in room temperature for hours, dissections of leg and foot were performed in those specimens. The tendon of FHL was crossing over the tendon of FDL from posterosuperolateral to the medial side just beneath the navicular bone and approximately $2.5 \mathrm{~cm}$ laterally. The morphologic features of intertendinous connections were investigated at this crossing point which is named as "Henry's knot" and where the tendons course in a common synovial sheath.

Results: Tendinous connections between FHL and FDL tendons were found in 18 cases (72\%) out of 25 lower extremities dissected. Intercrossing tendon connection (Type III) was detected in 8 cases (44\%), only a tendinous slip crossing from FHL to FDL tendon (Type I) was observed in 10 cases (55\%). However, a connection only from FDL to FHL tendon (Type II) was not encountered in our dissections. In addition, in $40 \%$ of dissected cases, quadratus plantae muscle was observed to have a substantial tendon commencing from the calcaneus and coursing medial to the muscle and this tendon was seen to access to the branching site of FDL tendon from lateral and intermingle it from above. The tendon was seen to slip into two parts and coursed separately towards the tip of the toes. In summary, the tendon connections between FHL and FDL coursed medial to the quadratus plantae muscle, contributed to the flexion of 2 nd and 3rd toes, and tendon of quadratus plantae together with FDL formed a functionally common tendon complex.

Conclusion: At the crossing point of FHL and FDL in the sole of the foot where it is named as Henry's knot or chiasma plantare, it was reported in classical anatomical textbooks that there may be various types of intertendinous connections, however there is no satisfactory information on their form, structure, frequency and function. The tendinous connections observed between FHL, FDL and quadratus plantae muscles in the feet without any congenital anomaly are probably because of their development from the same common embryologic origin. Relatively strong intertendinous connections may be encountered in substantial cases at the crossing site of FHL and FDL tendons in the foot. This connection may cause tenodesis effect when the function of the main tendon is lost. Various types of structural changes of tendons should be remembered by anatomists and orthopaedic surgeons dealing with foot surgery especially in the diagnosis and treatment of muscle, tendon or neural injuries following atraumatic or penetrating lesions of the sole of the foot. Such intertendinous crossings may also cause problems in tendon transfer procedures as well as in pes equinovarus surgery and may ease the explanation of complications.

Keywords: Tendinous connections, foot, flexor hallucis longus muscle, flexor digitorum longus muscle

\section{P-126}

\section{Contribution of peroneus brevis muscle to} the extension of 5 th toe

\section{Tugtag $\mathrm{B}^{*}$, Gumusalan $\mathrm{Y}^{*}$, Uzel $\mathrm{M}^{* *}$}

*Department of Anatomy, Faculty of Medicine, Fatih University, Istanbul, Turkey; ${ }^{*}$ Department of Orthopaedics and Traumatology, Faculty of Medicine, Kahramanmaras Sütcü Imam University, Kahramanmaras, Turkey

Objectives: Functional loss may be encountered in muscletendon units in the toes due to various reasons. Understanding of the anatomical origin and variations of muscles is important in the evaluation of functional losses and treatment. In this study, anatomical features and variations of muscles extending to the 5 th toe and causing its extension were investigated. 
Methods: Dissections of the feet were performed in lower extremities amputated by the Department of Orthopaedics and Traumatology. Tendon variations, muscle-tendon extensions and muscular morphologies were classified according to origins-insertions and the measurements were recorded.

Results: Tendinous attachments extending from the peroneus brevis $(\mathrm{PB})$ muscle to the dorsal aponeurosis of the 5 th toe were observed in 8 cases when 25 lower extremities were dissected. These tendons were in $3 \times 1,2 \times 1$ and $3 \times 2 \mathrm{~mm}$ dimensions. In addition, some tendons were seen to be divided from $\mathrm{PB}$ as two separate tendons approximately $10 \mathrm{~cm}$ proximal to the metatarsophalangeal joint and some fibers were observed to attach to the lateral aspect of the tendon of extensor digitorum longus (EDL) muscle extending to the 5th toe. In such cases, when $\mathrm{PB}$ muscle and/or its tendinous extension was pulled proximally, it was able to extend the 5 th toe.

Conclusion: It has been reported in the literature that the primary extensor muscle of the 5 th toe is EDL and it develops from extensor hallucis longus muscle together with $\mathrm{PB}$ and peroneus tertius (PT) muscles. However, PT does not contribute to the extension of the toe since it inserts to the dorso-medial aspect of 5 th metatarsal bone. On the other hand, PB causes eversion of the foot, thus contributes slightly to the extension of 5th metatarsal bone, but does not send extensor tendon bands to 5 th toe. In our dissections, additional tendon bands arising from PB muscle and extending distally were observed with a rate of $32 \%$ and inserted to the dorsal aponeurosis of the 5 th toe. When traction was applied to PB tendon, slight extension was observed in the 5 th toe. These additional tendons may cause partial extension of the 5 th toe in cases of break up or absence of EDL tendon or tenodesis effect which may prevent the flexion posture of the 5 th toe. The origination of muscles from the common source and extending from the anterior and peroneal compartments of the leg to the 5 th metatarsal and phalanges accounts for the reason of frequent existence of anatomic variations in this region. As the consequence of developmental abnormalities, these anatomical variations should be borne in mind during the evaluation of functional losses, formational disorders and other abnormalities of the 5 th toe.

Keywords: Peroneus brevis muscle, 5th toe, foot, extension, tendon insertional anomaly

\section{P-127}

Protective effects of quercetin against arsenic-induced testicular damage in rats

$\underline{\text { Baltac1 BB*}}$, Uygur R*, Caglar $V^{*}$, Aktas $C^{* *}$, Aydin $\mathrm{M}^{* * *}$, Ozen $\mathrm{OA}^{*}$

*Department of Anatomy, Faculty of Medicine, Namik Kemal University, Tekirdag, Turkey; **Department of Histology, Faculty of Medicine, Namik Kemal University, Tekirdag, Turkey; ***Department of Biochemistry, Faculty of Medicine, Namik Kemal University, Tekirdag, Turkey

Arsenic is a toxic and carcinogenic substance. Arsenic is known to have negative effects on male reproductive functions. In this study, protective effects of quercetin against arsenic-induced testicular damage in rats were investigated. In this study, 27 Sprague-Dawley male rats were divided into three groups. Control group (10 ml/kg/day SF), arsenic group (10 mg/kg/day sodium arsenite), arsenic + quercetin group $(10 \mathrm{mg} / \mathrm{kg} / \mathrm{day}$ sodium arsenite $+50 \mathrm{mg} / \mathrm{kg} /$ day quercetin). At the end of the 15 days, rats were sacrificed. Testis tissues were collected and stained with hematoxylin and eosin for histopathological analysis. Apoptotic cells were studied by using TUNEL assay. Cell proliferation was studied by PCNA method. The activities of superoxide dismutase (SOD), catalase (CAT), and glutathione peroxidase (GSH-Px) as well as malondialdehyde (MDA) were measured by spectrophotometry. As a result of exposure to arsenic in rat, our study showed that reduced germ cells and degeneration of seminiferous tubules, increase TUNEL-positive apoptotic cells in the walls of the seminiferous tubules and decreased PCNA-positive cells in testes by histopathological methods. After quercetin treatment, we have demonstrated that structural deterioration in testes was ameliorated, decreased TUNEL-positive apoptotic cells, increased PCNApositive cells. After arsenic exposure; decreased SOD, CAT and GSH-Px activities, increased in the MDA levels in testes tissues was detected by biochemical analyses. After quercetin administration, an increase of SOD, CAT and GSH-Px activity and a decrease of MDA levels were determined. These data suggested that quercetin has protective effects against arsenicinduced testicular damage by decreasing morphological damage, apoptosis, lipid peroxidation, and oxidative stress.

Keywords: Arsenic, quercetin, testis, apoptosis, oxidative stress

\section{P-128}

\section{Evaluation of morphometric features of temporomandibular joint by using cone-beam computed tomography}

\section{Kolsuz E*, Bilecenoğlu B**, Orhan KS}

*Department of Oral and Maxillofacial Radiology, Faculty of Dentistry, Ankara University, Ankara, Turkey; ${ }^{*}$ Department of Anatomy, Faculty of Dentistry, Ankara University, Ankara, Turkey; ${ }^{* *}$ Department of Oral and Maxillofacial Radiology, Faculty of Dentistry, Ankara University, Ankara, Turkey

Since the middle of twentieth century mandibular ramus osteotomy has become a preferred surgical procedure for the correction of various jaw deformities. Most important postoperative result of mandibular ramus osteotomy is the changes in temporomandibular joint (TMJ) and these changes may result in TMJ disfunction. Altough many studies have reported various changes in condylar and disc positions after mandibular osteotomies by different imaging techniques, there are less information about TMJ morphometric features. Aim of this study is to define morphometric features of TMJ by using cone-beam computerized tompgraphy (CBCT) in patient without TMJ complaints. Fort his purpose image reconstructions were obtained and prepared by using a Planmeca ProMax ${ }^{\circledR}$ 3D Max CBCT (Planmeca Oy, Helsinki, Finland). All the patients 
included in the study has no TMJ or orthodontic complaints and various measurements regarding TMJ was done on previously taken images. Images of 20 male and 20 female patients were viewed randomly by two observers three times and following measurements were made. In coronal sections; angle between condyle long axis-horizontal axis, angle between ramus long axis-vertical axis, angle between condyle long axis-ramus long axis, angle between condyle long axis-vertical axis, angle between ramus long axis-horizontal axis, latero-medial length of condyle, distance between lateral pole of condyle and mandibular fossa, distance between medial pole of condyle and mandibular fossa, distance between tip of condyle and mandibular fossa. In sagittal sections; distance between tip of condyle and mandibular fossa, distance between mid-point of condyle and tip of condyle and distance from mid-point of condyle to mandibular fossa. In axial sections the angle between each condyles long axis was measured. According to our results the changes in TMJ could be easily understood after mandibular ramus osteotomies and especially our results are very valuable for preoperative assesment of TMJ prosthesis patients.

Keywords: Temporomandibular joint, cone-beam computerized tomography, mandibular ramus osteotomy

\section{P-129}

Size and course of the inferior gluteal nerve in fetal period

\section{Candan B, Sulak O}

Department of Anatomy, Faculty of Medicine, Suleyman Demirel University, Isparta, Turkey

Objectives: The aim of this study is to determine size and course of the inferior gluteal nerve during the fetal period.

Methods: 400 inferior gluteal nerve, obtained from 200 human fetuses, aged between 9-40 weeks were used in this study. At first, gluteus maximus muscle was retracted bilaterally and the inferior gluteal nerve was exposed. Exit location, thickness, and length of the inferior gluteal nerve till the point it entered the gluteus maximus muscle and variations of the inferior gluteal nerve were determined. Also the inferior gluteal nerve distribution in the gluteus maximus muscle was evaluated.

Results: The inferior gluteal nerve exited the medial of the sciatic nerve and passing above the piriformis muscle then entered the gluteus maximus muscle. Only one case, the inferior gluteal nerve exited the lateral of the sciatic nerve and passing through the piriformis muscle and entered to the gluteus maximus muscle $(0.2 \%)$. Sizes of inferior gluteal nerve were increased with gestational age. The inferior gluteal nerve entered the gluteus maximus muscle $1 / 4$ of the lower lateral part of the muscle and then it distributed in the muscle in all cases.

Conclusion: The knowledge of course, development and variations of inferior gluteal nerve is important in surgical approach, diagnosis and treatment of the related diseases. When the studies carried out so far were evaluated it was seen our study is the first one during fetal period. It was concluded that our study will constitute the basis on this subject for future studies.

Keywords: Variation, fetus, inferior gluteal nerve

\section{P-130}

Double-headed piriformis muscle in fetal cadaver: 3 case report

Candan B, Sulak O

Department of Anatomy, Faculty of Medicine, Suleyman Demirel University, Isparta, Turkey

Objectives: The aim of this report is to determine of the incidence of double-headed muscle clinically and evaluation of variation of the piriformis muscle during the fetal period.

Methods: Case have been identified during the course of a master thesis entitled "Development of sciatic nerve during fetal period" which was carried out of 200 (103 males and 97 females) human fetuses aged between 9-40 weeks of gestation. Variations were recorded and photographed.

Results: When the gluteus maximus muscle was retracted, the piriformis was observed as double-headed; in one case on the right side $(0.5 \%)$ and in two cases on the left side ( $1 \%)$ during dissection. Both of the muscle heads were uncovered, and their locations were identified. We observed two head of the piriformis muscle which start from the front face of the Sacrum, unit the tendons and end at the greater trochanter. And we observed the sciatic nerve exits below the double-headed piriformis muscle in 3 cases.

Conclusion: In previous studies it has been defined that blood vessels and nerves of this region emerge above, middle and below the muscle. Thus, any anatomical variation of this muscle has clinical importance. In the available literature, doubleheaded piriformis muscle is rare and if found, could be a cause for the undiagnosed chronic pain in the gluteal region, as this muscle may compress the sciatic nerve. Submission of variations of the piriformis muscle is important for piriformis syndrome and surgery of gluteal region. We hope that our findings may guide for future studies.

Keywords: Variation, fetus, piriformis muscle, nervus ischiadicus

\section{P-131}

$2.45 \mathrm{GHz}$ radio frequency radiation exposure-induced changes on rat testes and protective effects of vitamins $\mathrm{E}$ and $\mathrm{C}$

Bilkay $\mathrm{C}^{*}$, Ozguner $\mathrm{G}^{*}$, Dursun $\mathrm{A}^{*}$, Comlekci $\mathrm{S}^{* *}$, Deniz $\mathrm{K}^{* * *}$, Erten $\mathrm{S}^{* * *}$

*Department of Anatomy, Faculty of Medicine, Süleyman Demirel University, Isparta, Turkey; **Department of Telecommunications, Faculty of Engineering, Süleyman Demirel University, Isparta, Turkey; $* * *$ Department of Pathology, Faculty of Medicine, Erciyes University, Kayseri, Turkey

Objectives: In this study, we aimed to investigate $2.45 \mathrm{GHz}$ radio frequency radiation (EMR) exposure induced changes on rat testes and protective effects of vitamins $\mathrm{E}$ and $\mathrm{C}$.

Methods: 28 male Sprague-Dawley rats were used in this study. Rats were divided into three groups: Group I: Control group, (same amount of water given to the group III by oral gavages), 
Group II: exposed to $2.45 \mathrm{GHz}$ EMR, Group III: exposed to $2.45 \mathrm{GHz}$ EMR + Vitamins E and C (50 mg vitamin E + $200 \mathrm{mg}$ vitamin $\mathrm{C} \mathrm{mg} / \mathrm{kg}$ body weight / day, oral gavages). Group II and III were exposed to $2.45 \mathrm{GHz}$ EMR, for 60 minutes every day for 30 days. Vitamin $\mathrm{E}$ and $\mathrm{C}$, was given 1 hour before the EMR application for Group III. The rats were sacrificed under general anesthesia at the end of 30 days period, and the testes were dissected. Routine tissue procedure was applied and the sections were stained with hematoxylin-eosin (H-E).

Results: The weights and volumes of the testes were reduced in the EMR exposed groups. In histopathological examinations, diameter of tubulus seminiferus and Johnsen scores were reduced in the EMR exposed groups. However, no statistically significant difference was observed between groups. It is determined that, the changes were lower in vitamin $\mathrm{E}$ and $\mathrm{C}$ administered groups when compared to group two. There was no statistically significant difference was observed in Leydig cell scores.

Conclusion: EMR applications causes changes in rat testes and, vitamin $\mathrm{E}$ and $\mathrm{C}$ have some protective effects.

Keywords: Testis, rat, $2450 \mathrm{mhz}$ radio frequency, vitamin E, vitamin $\mathrm{C}$

\section{P-132}

Examining bulbus oculi's morphometric characteristics among cataract patients

Ateşoglu S*, Senol D**, Balsak S*, Alakus MF*, Ozbag D**

*Diyarbakır Gazi Yaşargil Training and Research Hospital, Diyarbakır, Turkey; **Department of Anatomy, Faculty of Medicine, Inönü University, Malatya, Turkey

Objectives: This study aimed at retrospectively investigating how such morphometric measures of right bulbus oculi (RBO) and left bulbus oculi (LBO) as camera anterior, lens, vitreous, and total axial length varied among cataract patients by age and gender.

Methods: This study employed the camera anterior, lens, vitreous, and total axial length measures obtained from the RBOs and LBOs of the patients coming to Diyarbakır Gazi Yaşargil Training and Research Hospital Eye Diseases Clinic for cataract operation in preop. Based on such measures, the data were subjected to t-test and correlation analysis. IBM SPSS Statistics 22.0 for Windows was used in analyses.

Results: The data obtained from 118 subjects 63 (53\%) of whom were male, and 55 (47\%) of whom were female were included in analysis. While the average age of the males was 70 \pm 11 , that of the females was $70 \pm 13$. The t-test indicated that vitreous and total axial length obtained from $\mathrm{RBO}$ and total axial length obtained from LBO varied significantly by gender, but camera anterior and lens obtained from RBO and LBO and vitreous obtained from LBO did not vary significantly by gender. The correlation analysis demonstrated that camera anterior and vitreous obtained from RBO and LBO had a poor negative relationship with age; and lens obtained from RBO and LBO had a poor positive relationship with age.
Conclusion: It was concluded that such RBO and LBO measures as camera anterior, lens, vitreous, and total axial length had a poor relationship with age and gender.

Keywords: Camera anterior, lens, vitreous, axial length, age, gender

\section{P-133 \\ The history of anthropometric studies from past to present}

$\underline{\text { Senol D, }}$ Cay M, Karatas T, Ozbag D

Department of Anatomy, Faculty of Medicine, Inonu University, Malatya, Turkey

The first modern studies on human anthropometry started to appear in the 18th century. C.F. Jampert (1754), G.L. de Buffon (1749-67), and Philibert Gueneau de Montbeillard (1777) left their marks in this field with the study dealing with the physical development of children, the study revealing the differences between cross-sectional and longitudinal studies, and the first longitudinal study of growth respectively. In the late 1800s, Cesare Lombroso and Eugene Vidocq supported their criminal studies with anthropometry. Quetelet was one of the first people launching the evaluation of anthropometric data via mathematical and statistical methods in the 19th century. In Turkey, the first study involving anthropology and focusing on human being directly was the work of Şemsettin Sami entitled "Insan [Human Being]" published in the 19th century. The oldest study about the growth and development of Turkish children was carried out by Nafi Atıf Kansu in 1917. Nurettin İrdelp, Neched Eumer, Mouchet, and others who were among the founders of the Turkish Anthropology Research Center conducted the study entitled "İstanbul'daki Türk, Rum, Ermeni ve Musevi çocuklarının neşvünemaları üzerine tetkikler [Examinations on the growths of the Turkish, Greek, Armenian, and Jewish children in Istanbul]" between 1926 and 1927 and published it Tïrk Antropoloji Mecmuast [Turkish Anthropology Journal] in 1927. In the study entitled "Üsküdar süt ve mektep çocukları dispanseri çalışmasından; çocuklarda büyüme nispetleri [Growth ratios of children based on the study conducted in Üsküdar Dispensary for nurslings and schoolchildren]" and carried out in 1938, S. B Tümay divided poor, middle class, and rich children into groups and evaluated them. Ş. A. Kansu, N. Gökçül, N. Çınar, K. Kökten, and M. Kinay contributed to the development of anthropometry with their studies in 1939. They were followed by N. İlbars and Z. Yalım in 1940. The studies until Yalım's research found out that height and weight values of the Turkish schoolchildren were much lower than the anthropometric values of their peers in the USA and Europe. Published between 1954 and 1957, the study of E. Y. Bostanci performed 35 anthropometric measurements on 832 male and 847 female schoolchildren in Ankara and evaluated such children in terms of various indices. This study was followed by Çiner who conducted an anthropometric study on 1838 women in the 20 to 40 age group in 1960 and Hertzberg et al. who carried out a study on 
915 Turkish soldiers within the scope of a NATO research in the same period (1960-61). The most recent study in this field is the one conducted by Erksin Güleç et al. on 2100 women and men across the country in 2004 to 2005.

\section{P-134}

\section{Examining the dynamic balance scores of individuals with different somatotype characteristics}

Senol D*, Ozbag D*, Kafkas ME**, Acak M**, Baysal O***, Sahin Kafkas $\mathrm{A}^{* *}$, Taskiran $\mathrm{C}^{* *}$, Cay $\mathrm{M}^{*}$, Yagar $\mathrm{D}^{* * *}$, Ozen $\mathrm{G}^{* *}$ *Department of Anatomy, Faculty of Medicine, Inönü University, Malatya, Turkey; **School of Physical Education and Sports, Inönü University, Malatya, Turkey; ${ }^{* *}$ Department of Physical Therapy and Rehabilitation, Faculty of Medicine, Inönü University, Malatya, Turkey

Objectives: This study aimed to investigate the influences of different somatotype characteristics of İnönü University School of Physical Education and Sports (SPES) students that did not have any symptom on their dynamic balance scores.

Methods: 179 students from different grades of İnönü University SPES participated in the study. The somatotype characteristics of all participants were calculated through the "Heath-Carter" formula. The dynamic balance scores of the participants were measured through the "Biodex Balance Simulator (Model: 945-302)”. Balance measurements were performed as overall balance, anterior/posterior balance, and medial/lateral balance. The first statistical operation performed in the study was the "Kolmogorov Smirnov" test aimed at determining whether or not the data were homogenous. After it was found that the data did not have a homogenous distribution, the "Kruskal Wallis H" test was performed for multiple group comparisons. The data obtained in the study were analyzed via SPSS for Windows 21.0. The research data were presented in terms of arithmetic mean (X), standard deviation (sd), and $\mathrm{p}<.05$ significance level.

Results: Based on the measurements performed, 13 different somatotype body types were determined. At the end of the dynamic balance measurements, it was seen that overall balance varied between 1.2 and 12.3 and had an average of 5.0 2.2 ; anterior/posterior balance varied between 0.7 and 8.2 and had an average of $3.7 \pm 1.6$; and finally medial/lateral balance varied between 0.7 and 11.1 and had an average of $3.4 \pm 1.9$. Furthermore, it was understood that the highest overall balance score was achieved by the individuals with the Mesomorph Endomorph somatotype; the highest anterior/ posterior balance score was achieved by the individuals with the Mesomorph Ectomorph somatotype; and the highest medial/lateral balance score was achieved by the individuals with the Mesomorph Ectomorph somatotype.

Conclusion: Based on the $\mathrm{p}$ values obtained in the present study, no significance difference was found between overall balance, anterior/posterior balance, and medial/lateral balance performances and somatotypes.

Keywords: Dynamic balance, somatotype, anthropometry

\section{P-135}

Examining the influence of somatotype differences on the isokinetic knee muscular force ratios of young people without any symptom

$\underline{\text { Senol D*}}$, Ozbag D*, Kafkas ME**, Acak M**, Baysal O**, Sahin Kafkas $A^{* *}$, Taskiran $C^{* *}$, Cay $M^{*}$, Yagar $D^{* * *}$, Ozen $G^{* *}$ *Department of Anatomy, Faculty of Medicine, Inonu University, Malatya, Turkey; **School of Physical Education and Sports, Inonu University, Malatya, Turkey; ${ }^{* *}$ Department of Physical Therapy and Rehabilitation, Faculty of Medicine, Inonu University, Malatya, Turkey

Objectives: This study aimed to investigate the isokinetic knee muscular force ratios of İnönü University School of Physical Education and Sports (SPES) students that had different somatotype characteristics but did not have any symptom.

Methods: 179 students from İnönü University SPES that did not have any illness participated in the study. Among the students participating in this study, height and weight values, skinfold thickness in four different places (triceps, subscapular, suprailiac, calf), bone width in two different regions (knee and elbow width), and two circumference values (arm, calf) were calculated. The somatotype characteristics of all participants were calculated through the "Heath-Carter" formula. The knee flexion and extension muscular forces of the participants were measured via the "Biodex System 3" Isokinetic test and the Exerciser (Model: $830-220)$ at such different angular speeds as $90 \mathrm{o} / \mathrm{s}, 120 \mathrm{o} / \mathrm{s}$, and $150 \mathrm{o} / \mathrm{s}$. The dominant and the non-dominant knee muscular forces of all participants were tested. The "Kolmogorov Smirnov" test was performed for determining whether or not the data were homogenous. After it was found that the data did not have a homogenous distribution, the "Kruskal Wallis H" test was performed for multiple group comparisons. The data obtained in the study were analyzed via SPSS for Windows 21.0. The research data were presented in terms of arithmetic mean $(\mathrm{X})$, standard deviation (sd), and $\mathrm{p}<.05$ significance level.

Results: It was determined that there were 13 different somatotype groups. It was found out that the individuals with the Balanced Mesomorph somatotype had the highest isokinetic knee muscular force ratio at all angular speeds; they were followed by the individuals with the Endomorph Mesomorph somatotype; and the individuals with the Mesomorph Ectomorph somatotype had the third highest isokinetic knee muscular force ratio. No significant difference was found in regard to the other groups. Based on the Kruskal Wallis H-Test analysis, it was seen that the somatotype difference led to a significant difference in dominant and non-dominant knee flexion peak force values at the $90^{\circ}$ angular speed; in dominant knee flexion and extension peak force values at the $120^{\circ}$ angular speed; and in dominant flexion and extension peak force values at the $150^{\circ}$ angular speed.

Conclusion: It was understood that the participants having the Balanced Mesomorph somatotype among different somatotype characteristics had higher isokinetic knee muscular force ratios in comparison to the other groups. Thus, it may be accepted as a criterion for selecting athletes for branches that require force in particular. However, there is still a need for research to be conducted on larger sample groups or athletes of different levels.

Keywords: Isokinetic force, anthropometry, somatotype, flexion, extension 


\section{P-136}

A survey of scientific publications in the field of anatomy conducted in Turkey over 2000-2014

Metin Tellioglu A, Karakas S, Polat AG

Department of Anatomy, Faculty of Medicine, Adnan Menderes University, Aydin Turkey

Objectives: In our study, we aimed to reveal the statistical data of the international scientific publications in the field of anatomy made in our country since 2000 .

Methods: Statistical data at the Turkey based publications, which have at least one anatomy specialist identified and have been published in journals at the ISI Web of Science database and covered by the SCI (Science Citation Index) and SCI-E (Expanded), were screened. The time period for the study is between the years 2000 and 2014. Number of publications by year, rate of increase in the number of publications, classification of broadcast (articles, papers, etc.), category of publications, and their citation rates were recorded. We determined the top 10 journals which have the most comprising publications, and the top 10 universities which facilitated these publications.

Results: Since 2000, we reached a total of 1385 publications in the field of anatomy. These publications are comprised of 1,285 original articles; 35 meeting presentations, 32 abstracts, 27 letters to the editor, and 23 compilations. Regarding to the number of publications, there is a gradual decrease in recent years, despite a significant increase observed in year 2009. In Turkey, ??the most published and cited categories are the surgical anatomy, morphology and neuroscience. The top three journals with highest number of publications are Surgical and Radiologic Anatomy, Saudi Medical Journal, and Clinical Anatomy. The top three publishing institutions are Ankara University, Hacettepe University and Ege University.

Conclusion: Our study provides us an understanding of the topics anatomists have favored to study recent years. A large number of published research articles are about surgical anatomy, morphology and neuroscience. Obviously, the quality of publications and their number of citations are more important than their scalar quantity. We believe the future studies should focus on the multidisciplinary areas with more citation prospects.

Keywords: Anatomy, scientific publications, SCI, web of science

\section{P-137}

Views of Adnan Menderes University Faculty of Medicine 2nd year students about anatomy education

Metin Tellioglu A, Karakas S, Gogebakan K, Polat AG

Department of Anatomy, Faculty of Medicine, Adnan Menderes University, Aydin Turkey

Objectives: The method as to how the anatomy education in medical schools ought to be and which education models to be selected as to reach the optimum way to present the course is a topic of discussion locally and globally. Therefore, we set our sights on getting to know the thoughts of the students on anatomy education who are looking to practice their knowledge clinically in the future.

Methods: The study encompassed students in their second years who have completed their full courses of anatomy education in 2013-2014 academic year of Adnan Menderes University Medicine Faculty. Out of 138 students, 120 of them (64 females, 56 males) accepted to participate with in the study. A survey of 25 questions which inquired parameters such as instruction methods, resources used, time allocated to anatomy etc. and the students were asked to answer those questions.

Results: While 109 of the group (90.8\%) consider the anatomy courses to be essential, $11(6.7 \%)$ concur other wise. The most important factor in anatomy courses to be efficient was deemed to be the instructor $(57.5 \%)$ and the use of supplementary materials such as 3D images $(89.2 \%)$, video instructions $(87.5 \%)$ and radiologic images $(70 \%)$ shared high ratios as well. A total of 116 students $(96.7 \%)$ shared a request for the courses to be held with in smaller groups and while 61 participants $(50.8 \%)$ would rather the application courses on cadavers, another 39 (32.5\%) preferred models over those. 104 students not edthattheydid not utilize printed materials of the library to study, 85 of them declared the same with online databases and there were 105 students who spoke about using books at home along with atlas to study.

Conclusion: So as to serve the anatomy education at its optimum, the possible innovations with regards to the perspective of the individuals subject to this very education should be taken into consideration. In our modern world which very other day with technological advancements, finest education for the branch of anatomy would be procured via help of technological resources.

Keywords: Anatomy education, student views, survey

\section{P-138}

\section{Period of plastinated cadaver in medical education}

Uludag S, Senol D, Cay M, Ozbag D

Department of Anatomy, Faculty of Medicine, Inönü University Malatya, Turkey

Keystone of medical education is Anatomy. On the other hamd, the must of anotomy education is to conduct it over cadavers. Most medicine curriculum depends on cadavers to teach macroscopic anatomy. The shortages experienced in the supply of cadavers is a known fact for the anatomy world. The reason for this is the fall in the number of unclanatomy. However, a way of reversing this situation in our country has emerged recently through plastinated cadavers. Plastination is a method where anatomical samples are freed from fats and water and coated with a polymer substance, that is, tissue fat and water are replaimed cadavers and decrease in cadaver donation and one of the probable solutions is to increase the number of cadaver donation. The question how long medical education can be maintained at our country with insufficient 
cadavers is asked loudly for a long time in the world of aced by durable polymer. This method was found by Prof. Dr. Gunther von Hagens of Heidelberg University in 1978. In the last 20 years, plastination has become an important tool to protect well dissected species and human parts and organs. To increase the use life of dissected cadavers used for a long time by plastinating them shall provided various benefits by keeping them in more hygienci conditions and away from damages of formaldehyde. Thus, more cleaner and suitable environment may be provided for lectures. We can be released from more difficult traditional cadaver keeping methods and their use in classes through plastinated cadavers. Formalin evaporating from the tissues causes miscellaneous damages on eyes, skin and mucosa. Further, it deteriorates the air in the environment and influences the quality of education. Furthermore, tissues and organs being far from their natural appearance prevents the opportunity of holding them for macroscopic examination. The students lose interest in the course due to these reasons and this causes them to be unsuccessful. In addition, the students and academic staff ask for an odorless, dry, robust medium which lasts longer and is more healthier which they shall be able to use without needing protective equipment such as gloves and masks, and a real educational material. In the last decade, the role of anatomic training has significantly changed in the curriculum for undergraduate education in medicine. Plastination is considered to be the most valid method to increase the storage, protection and use time of the cadavers used as educational material and to introduce the best samples as "ideal educational material". Recently, plastinated cadaver is offered as a revolution to the students for human and veterinary anatomy. While the discussion about the application purposes of and remarks on plastinated cadavers increase, the exhibition of plastinated cadavers and organs attracts the interest of millions of people all around the world. Therefore, ethic rules must also be considered in the plastination process.

Keywords: Plastinated cadaver, plastination, anatomy

\section{P-139}

The comparative analysis of the rolling effects of exercises of different frequency and intensity on bone mineral density in different anatomic regions of body

Korkmaz MF*, Cetin $A^{* *}$, Ates $M^{* *}$, Oztanır MN**, Karatas T**, Beytur L**, Cay $M^{* *}$, Senol D**

*Department of Orthopaedics and Traumatology, Faculty of Medicine, Inonu University, Malatya, Turkey; **Department of Anatomy, Faculty of Medicine, Inonu University, Malatya, Turkey

Objectives: Various studies have showed that physical activity has a positive effect on bone mass during growth and even adolescence. However, details about the nature and size of such relationship are still unclear. This study aimed to investigate the rolling effects of exercises of different frequency and intensity on bone mineral density (BMD) in different anatomic regions of body.

Methods: Among the national team athletes who danced halay and played horon (each group consisted of 26 individuals), those who were not in the 20-27 age range were excluded from analyses. In this way, 3 groups (13 horon players 6 of whom were female and 7 of whom were male [average age $22 \pm 2.3$ years; age range 20-27 years]; 20 halay dancers 11 of whom were female and 9 of whom were male [average age $22 \pm 1.8$ years; age range 20-26 years]; and 19 individuals 10 of whom were female and 9 of whom were male who were not involved in any folk dancing [average age $22 \pm 1.7$ years; age range $20-25$ years]) were included in the study. The paired-samples t-test, the one-way ANOVA, and the Tukey's test were employed to determine the main effects and combined effects on BMD.

Results: There was no significant difference between subjects in terms of age, weight, and height. When the athletes older than 20 who were engaged in sports for 6 to 10 years were considered, no difference was found between the horon players and the halay players in terms of age, weight, height, waist circumference, and right and left calf circumferences ( $p>0.05)$. While no difference was found between the horon players and the halay dancers in terms of lumbar and proximal femur values, the horon players were found to have statistically significantly higher forearm BMD values $(0.56 \pm 0.09)$ (p: 0.009). The lumbar total BMD was found to be the highest in the group not engaged in sports $(1.02 \pm 0.10)$, and it was statistically significant (p:0.004).

Conclusion: Twisting forces in exercises affect the modeling and remodeling of bones as well as their strength and tension through imposing more force on bones besides leading to an increase in muscle mass and neural maturation. Thus, incremental loading stimulates bone modeling, and cortical bone shape changes result in increase in trabecular BMD and bone strength. Based on the obtained data, it was found out that exercises of different frequency and impact had different effects on BMD in different anatomic regions of the body. There is a need for more research to determine the optimal dose of physical activity for children and adults in order to optimize bone mass acquisitions. Providing important evidences, these results may contribute to health institutions and organizations in determining the types of exercises to be done for obtaining the peak BMD.

Keywords: Bone mineral density, exercises of different frequency and intensity, rolling effects

\section{P-140}

\section{Protective and therapeutic effects of dexpanthenol on isoproterenol-induced cardiac damage in rats}

Kalkan $\mathrm{F}^{*}$, Disli OM**, Parlakpinar $\mathrm{H}^{* * *}$, Tanriverdi $\mathrm{LH}^{*}$, Polat $\mathrm{A}^{* * * *}$, Cetin $\mathrm{A}^{* * * * *}$, Vardi $\mathrm{N}^{* * * * *}$, Acet $\mathrm{A}^{* * *}$

*Under-Graduated Student, Faculty of Medicine, Inonu University, Malatya, Turkey; ${ }^{* *}$ Department of Cardiovascular Surgery, Faculty of Medicine, Inonu University Malatya, Turkey; $* * \star$ Department of Medical Pharmacology, Faculty of Medicine, Inonu University, Malatya, Turkey; $* * * *$ Department of Physiology, Faculty of Medicine, Inonu University, Malatya, Turkey; $* * \star \star *$ Department of Histology and Embryology, Faculty of Medicine, Inonu University, Malatya, Turkey 
Objectives: To explore the protective and curative effects of dexpanthenol (DEX) on isoproterenol (ISO)-induced cardiac damage in rat heart.

Methods: Forty rats were randomized into four groups $(n=10)$ : control group; ISO group (150 mg/ $\mathrm{kg})$; DEX+ISO group (DEX $250 \mathrm{mg} / \mathrm{kg}$ administrated 30 minutes before the repetitive two single dose of ISO in first two days, than DEX continued for 2 days); ISO+DEX group (2 days after the repetitive two single dose of ISO and DEX continued for 2 days). Rats were monitored for mean arterial blood pressure, heart rate, $\mathrm{O} 2$ saturation, and electrocardiography. Heart tissue levels of malonilaldehyde (MDA), superoxide dismutase (SOD), catalase (CAT), glutathione peroxidase (GPX), and reduced glutathione (GSH) were determined.

Results: Blood pressure and $\mathrm{O} 2$ saturation values indicated a significant decrease in the ISO group compared to the control group. T wave negativity was observed in 6 of 10 rats in the ISO group, in 1 of 10 rats in the ISO+DEX group, and none of 10 rats in the DEX+ISO group. SOD and GPX levels were significantly lower in the ISO group compared to the control and the DEX+ISO groups. CAT levels were significant decrease in the ISO group compared to the control group. ISO administration induced morphological alterations such as disorganization of cardiomyocytes, loss of myofibrils and cytoplasmic vacuolization in the heart. On the other hand, the histological damage was significantly decreased in the ISO+DEX and DEX+ISO groups when compared to the ISO group.

Conclusion: This study implies the cardioprotective effects of DEX on ISO-induced cardiotoxicity.

Acknowledge: This study was supported by TUBITAK (The Scientific and Technological Research Council of Turkey, project number: 2209/A-2012, belongs to Ferhat Kalkan).

Keywords: Isoproterenol; heart; dexpanthenol; cardiotoxicity.

\section{P-141}

\section{Crista galli pneumatization in the pre-adult and adult stages}

Tetiker $\mathrm{H}^{*}$, Kosar $\mathrm{MI}^{*}$, Cullu $\mathrm{N}^{* *}$, Sahan $\mathrm{M}^{* * *}$, Gencer CU* *Department of Anatomy, Faculty of Medicine, Mugla Sitki Kocman University, Mugla, Turkey; ${ }^{*}$ Department of Radiology, Faculty of Medicine, Mugla Sitki Koçman University, Mugla, Turkey; ${ }^{* \star \star}$ Department of Otolaryngology, Faculty of Medicine, Mugla Sitki Koçman University, Mugla, Turkey

Sinonasal region in human was one of the region that commonly showed anatomical variations. Anatomical variations in the region might be easily diagnosed by paranasal CT evaluation. One of these variations was the crista galli pneumatization. In recent years, there has been opinions that support pneumatization origined from frontal sinus. In this study, we planned to evaluate that presentation of crista galli pneumatization was variety or not in pre-adult and adult period. In this retrospectively designed study, 218 coronal paranasal CT images that were filmed between 2012-2013 years were evaluated. Patients were divided into two groups according to the age of eighteen under or over. In the group under the age of eighteen, 97 cases, ratio of pneumatization was detected $2.1 \%$, in the group over the age of eighteen, 121 cases, crista galli pneumatization were detected $15.7 \%$. According to these results, crista galli pneumatization was found to be increased in adulthood. According to the frontal sinus that was a rudimentary state during childbirth was firstly detected in 6 years as a radiographic and reached on main size in puberty, this increase was parallel to the development of the frontal sinus. Consequently, this was supported the opinions that the crista galli pneumatization might be sourced from frontal sinüs.

Keywords: Crista galli, paranasal sinus, pneumatization, computed tomography

\section{P-142}

\section{Relationships between the upper extremity anthropometric measurements}

Aygun $\mathrm{D}^{*}$, Erdem $\mathrm{H}^{* *}$, Ozandac $\mathrm{S}^{* * *}$, Kizılkanat E**, Boyan $\mathrm{N}^{* *}$, Oguz $\mathrm{O}^{* *}$

*Adana State Hospital-KETEM Polyclinic, Adana, Turkey; **Department of Anatomy, Faculty of Medicine, University of Cukurova, Adana, Turkey; $\star \star \star$ Department of Physiotherapy, Ministry of Family and Social Policies, Adana, Turkey

Objectives: This study was conducted to determine relationships of the second and fourth finger morphometric measurements with hand morphometric measurements and the values with the range of motion, length and circumference of upper extremity.

Methods: The anthropometric measurements were taken from 39 (female: 23, male: 16) students (dominant extremity) who are studying in Cukurova University Faculty of Dentistry, ages between 20-26. In our study upper extremity, arm span, hand, second and fourth finger lengthes, arm and fore arm circumference, hendbreadth, wrist diameter, wrist and finger normal range of motion measurements were taken. The stastical analyses, which were measured by caliper, tape and goniometer, were evaluated by SPSS 20.00 .

Results: The anthropometric measurements were found as: upper extremity length (female: $68.11 \pm 13.63 \mathrm{~cm}$, male: $76.35 \pm 3.40 \mathrm{~cm}$ ), arm span (female: $160.82 \pm 7.40 \mathrm{~cm}$, male: $176 \pm 6.94 \mathrm{~cm}$ ), arm circumference (female: $26.93 \pm 2.50 \mathrm{~cm}$, male: $30.92 \pm 2.71 \mathrm{~cm}$ ), fore arm circumference (female: $23.15 \pm$ $1.63 \mathrm{~cm}$, male: $25.88 \pm 1.94 \mathrm{~cm}$ ), handbreadth (female: $7.47 \pm$ $0.54 \mathrm{~cm}$, male: $9.14 \pm 2.71 \mathrm{~cm}$ ), hand length (female: $18.33 \pm 0.95$ $\mathrm{cm}$, male: $20.32 \pm 0.92 \mathrm{~cm}$ ), second finer length (female: $8.69 \pm$ $0.48 \mathrm{~cm}$, male: $9.53 \pm 0.47 \mathrm{~cm}$ ), fourth finger length (female: $9.08 \pm 0.59 \mathrm{~cm}$, male: $9.98 \pm 0.51 \mathrm{~cm}$ ), wrist diameter (female: $4.97 \pm 0.52 \mathrm{~cm}$, male: $5.74 \pm 0.28 \mathrm{~cm}$ ), wrist circumference (female: $15.40 \pm 0.86 \mathrm{~cm}$, male: $16.70 \pm 1.73 \mathrm{~cm}$ ), wrist flexion and extension normal range of motion values are respectively (female: $90.69 \pm 0.92^{\circ}, 71.82 \pm 2.01^{\circ}$ and male: $90.93 \pm 1.76^{\circ}$, $70.68 \pm 1.92^{\circ}$ ), metacarpophalangeal, proximal interphalangeal and distal interphalangeal range of motion values are respectively female: $92.4783 \pm 2.08^{\circ}, 118.52 \pm 2.64^{\circ}$ and $76.78 \pm 3.76^{\circ}$, male: $91.56 \pm 2.03^{\circ}, 118.87 \pm 1.97^{\circ}$ ve $\left.77.62 \pm 3.22^{\circ}\right)$. 
Conclusion: Although there are significant relationships between the dominant upper extremity lengthes, there is not a significant relationship between the upper extremity anthropometric measurements and normal range of motion values.

Keywords: Upper extremity, anthropometry, normal range of motion.

\section{P-143}

The morphometric measurements of the anatomical structures at the proximal end of the humerus

Onder $M^{*}$, Oguz Yolcular B**, Oguz N*

*Department of Anatomy, Faculty of Medicine, Akdeniz University, Antalya, Turkey; **Department of Biostatistic and Medical Informatics, Faculty of Medicine, Akdeniz University, Antalya, Turkey

Objectives: The morphological features of the anatomical structures at the proximal end of the humerus are significant in surgical applications and anthropology.So, morphometric measurements of the anatomical structures at the proximal end of the humerus were determined with MicroscribeG2X.Finding of the morphometric data is aimed.

Methods: In our study, we used 25 left and 25 right humerus from Akdeniz University Faculty of Medicine, Anatomy Laboratory. proximal end of the humerus ; width and length of the greater tubercle with the lesser tubercle,length of the humerus,sagittal, vertical and transverse diameter of anatomical neck with surgical neck and distance between greater tubercle and lesser tubercle were measured with Microscribe-G2X. Descriptive analysis was applied to dataset and the results were presented as mean \pm standard deviation. Independent Samples T test was performed for comparison of quantitative variables with normal distribution. Mann-Whitney $U$ test was used for comparison of quantitative variables with non-normal distribution. The overall data were analyzed with the Statistical Package for the Social Sciences (SPSS) 13.0 and a p-value greater than 0.05 was considered statistically significant.

Results: According to the measurements of the anatomical structures at the proximal end of the left and right humerus means and standard deviations were: The length of Humerus: on the left $29.58 \mathrm{~cm} \pm 2.13$ / on the right $30.95 \mathrm{~cm} \pm 1.66$. The distance between greater tubercle and lesser tubercle: on the left $2.17 \mathrm{~cm} \pm 0.27 /$ on the right $2.54 \mathrm{~cm} \pm 0.28$. The width of greater tubercle: on the left $2.61 \mathrm{~cm} \pm 0.40$ / on the right $2.86 \mathrm{~cm} \pm 0.37$. The width of lesser tubercle: on the left $1.59 \mathrm{~cm} \pm 0.35$ / on the right $1.76 \mathrm{~cm} \pm 0.32$. The length of greater tubercle :on the left $2.83 \mathrm{~cm} \pm 0.28$ / on the right $2.98 \mathrm{~cm} \pm 0.39$. The length of lesser tubercle: on the left $2.01 \mathrm{~cm} \pm 0.29$ / on the right $2.02 \mathrm{~cm} \pm 0.30$. The vertical diameter of anatomical neck: on the left 4.24 $\mathrm{cm} \pm 0.35$ / on the right $4.53 \pm 0.41$. The transverse diamater of anatomical neck: on the left $3.93 \mathrm{~cm} \pm 0.29$ / on the right 4.09 $\mathrm{cm} \pm 0.43$. The sagittal diameter of surgical neck: on the left 2.34 $\mathrm{cm} \pm 0.29$ / on the right $2.47 \mathrm{~cm} \pm 0.27$. The transverse diamater of surgical neck: on the left $2.56 \mathrm{~cm} \pm 0.26 /$ on the right 2.67 $\mathrm{cm} \pm 0.22$, respectively. The average of the distance between greater tubercle and lesser tubercle at the proximal end of right humerus was found statistically higher compared to the proximal end of left humerus (2.54 $\mathrm{cm} \pm 0.28 ; 2.17 \mathrm{~cm} \pm 0.27$; $\mathrm{p}=0.001)$.

Conclusion: We think that these measurement will be helpful for surgeons and anthropologists.

Keywords: Morphometry, anthropology, prosthesis. humerus, anatomical neck, surgical neck

\section{P-144}

\section{Conflicting uses in anatomy terminology}

Cetin $N^{*}$, Farımaz $M^{*}$, Bayko $S^{*}$, Akkasoglu $S^{*}$, Askit $\mathrm{C}^{* *}$, Sargon $\mathrm{MF}^{*}$

*Department of Anatomy, Faculty of Medicine, Hacettepe University Ankara, Turkey; **Department of Latin Language and Literature, Faculty of Language, History and Geography, Ankara University, Ankara, Turkey

Most common used languages in anatomy nomenclature are Latin and English. Terminologia Anatomica, which is used as a reference book in the science of anatomy standardizes the terms as much as possible and renews itself by revisions over the years. However, some contradictory usages of terminological aspect may arise. In particular; ambiguity, between basic sciences and clinical sciences, may occur. Considering the meanings of the words; incompatible situations may be encountered between the relevant anatomical structures and the names given to these formations. Additionally; several anatomical structures have given names which have no relations with their anatomical relations. When we look through the view of Latin terminologia, we found out that the mistakes are because of wrong adding. During this study Terminologia Anatomica and several clinical text books were compared with each other and a support was taken from a master specialized in Latin terminology. This study reviewed the conflicting usages in anatomical terminology and pointed out the occurrence of the misuses.

Keywords: Anatomy nomenclature, clinical terminology, latin

\section{P-145}

\section{Identification of the important anatomic landmarks during vestibular nerve dissection}

Yegin $\mathrm{H}^{*}$, Bahcelioglu $\mathrm{M}^{* *}$, Gozil R**, Calguner $\mathrm{E}^{* *}$, Goksu $\mathrm{N}^{* * *}$

*Otolaryngology and Head and Neck Surgery, Edirne City Hospital, Edirne, Turkey; **Department of Anatomy, Faculty of Medicine, Gazi University, Ankara, Turkey; ${ }^{* * *}$ Department of Otolaryngology and Head and Neck Surgery, Faculty of Medicine, Gazi University, Ankara, Turkey

The posterior cranial fossa and the cerebello-pontine angle with their complex structures require understanding of their detailed anatomy. The aim of this study was to examine the relationships of the superior petrosal vein, fissure veins, choroid plexus, superior cerebellar, anterior inferior cerebellar, posterior inferior cerebellar, vertebral, labyrinthine arteries to the cranial nerves 
(nervus intermedius, abducens, facial and vestibulocochlear nerves) of the middle complex. For this purpose, a retrospective study was conducted in Gazi University, Faculty of Medicine, Department of Otolaryngology and Head and Neck Surgery. The operational records of the 109 patients admitted to the hospital between 1994 - 2004 were evaluated to clarify the topographic relationships of the neurovascular structures. Meanwhile, the percentage of the unusual presence of the vertebral and inferior posterior cerebellar arteries in the middle complex area was noted. Also, the incidence of the important landmarks such as nervus intermedius, abducens nerve and choroid plexus were demonstrated. The topographic relations of the vestibulocochlear nerve and its cleavage feature were detailed regarding the neighboring vascular structures. In conclusion, the vascular and neural structures encountered during the vestibulocochlear nerve surgery carry special importance. Also, rare structures and variations found in the region have to always keep in mind otherwise they carry high risk of complications.

Keywords: Pontocerebellar triangle, middle complex arteries, middle complex veins, middle complex nerves, variation

\section{P-146}

\section{Bilateral variation of accessory palmaris longus}

Ozkan M, Kirazli O, Yildiz SD, Altay G, Tosunoglu E, Verimli U, Sehirli US

Department of Anatomy, Faculty of Medicine, Marmara University Istanbul, Turkey

We have detected a bilateral accessory muscle in the flexor compartment of a 40 years old $80 \mathrm{~kg}$ male cadaver during routine educational dissections in Marmara University School of Medicine Anatomy Department Laboratories. The muscle originates from the medial epicondyle of humerus, extends towards the flexor retinaculum and demonstrates a "Y" shaped bifurcation distal to the $1 / 3$ of its tendinous part. Belly of the muscle is observed to be located on the medial side of palmaris longus muscle. The muscle has a course deep to palmaris longus in the medial region of the forearm and inserts to flexor retinaculum. Belly of the muscle has a fusiform shape and 10 $\mathrm{cm}$ length having a tendinous continuation of $6 \mathrm{~cm}$. After this tendinous part, the muscle bifurcates into two parts $3 \mathrm{~cm}$ in length. The muscle is supplied arterially by the ulnar artery and it is innervated by the median nerve. To the best of our knowledge, there has been no such variation reported in the literature. We think that defining the anatomy of this muscle may contribute to the clinically important region in the literature.

Keywords: Palmaris longus, accessory muscle, variation

\section{P-147}

The anatomical false in Rembrant's picture which is called as Anatomy Lesson of Dr. Tulp

Bahsi I, Orhan M

Department of Anatomy, Faculty of Medicine, Gaziantep University, Gaziantep
Anatomical studies was perfomed as public in Netherlands in 17 th century were social activities which were made under the name of 'anatomical theater'. People could join these activities by paying certain fee. Rembrandt picturized Dr. Nicolaes Tulp who performs forearm dissection together with a group audience in his picture was named as 'Dr. Tulp's Anatomy lesson'. In the picture, it is seen that Dr. Tulp pulls via a forceps the muscle group on forearm to show the audience and he show fingers movements in his hand. This picture is considered as a precious work in terms of pictorial art. In this study, anatomical errors and variations were examined in the picture was aforementioned. These; 1 . It is thought that the muscle group which is located on forearm's proximal and shown as adherent to lateral epicondyle should be flexor digitorum superficialis which is adherent to medial epicondyle, and also it is thought that it's position was picturized as wrong. 2. It is thought that the muscle group which is located on forearm's proximal and is shown as adherent to lateral epicondyle is not a muscle group, it is flexor digitorum superficialis that is a single muscle. 3 . It is thought that white structure which is located on ulnar side of forearm's distal is a variation of ulnar nerve. 4 . It is thought that the muscle which is located the medial side of forearm's proximal and lie between lateral epicondyle and ulna was picturized as pronator teres. It thought that this muscle should be between medial epicondyle and radius was picturized wrong. 5. It is identified there is not palmaris longus which is absent $10 \%$ as anatomical variation. Consequently, there are anatomical wrongs in Rembrandt's picture. Despite this, artistic value of the picture is very high . It is understood that a person who is equipped in their field cannot look at with the eye of an anatomist; anatomical drawings are different from classical paintings drawings, it requires special skills also it is more valuable scientifically when drawings is made by person who has sufficient knowledge and experience in the field of anatomical drawings.

Keywords: Dr. Tulp's Anatomy Lesson, Rembrandt, forearm dissection

\section{P-148}

\section{Morphometric measurements of calcaneus; Boehler's angle and relationship of the bone with anteroposterior length}

$\underline{\text { Otag } \mathrm{I}^{*}}$, Tetiker $\mathrm{H}^{* *}$, Tastemur $\mathrm{Y}^{* * *}$, Sabanciogullari $\mathrm{V}^{* * *}$, Kosar $\mathrm{MI}^{* *}$, Cimen $\mathrm{M}^{* * *}$

*Vocational School of Health Services, Cumhuriyet University, Sivas, Turkey; ${ }^{* *}$ Department of Anatomy, Faculty of Medicine, Mugla Sitkı Kocman University, Muğla, Turkey; ${ }^{* * *}$ Department of Anatomy, Faculty of Medicine, Cumhuriyet University, Sivas, Turkey

Measurements of bones give us information about the individual and the population it belongs to. In these studies, in addition to head and pelvis skeleton, calcaneus bone is also one of the other studied body bones as it can be preserved well. In the present study, 65 (35 right-30 left) well-preserved calcaneus bones were evaluated in Anatomy Laboratories of Cumhuriyet University and Mugla Sitkı Kocman University. With these 
bones, 10 linear measurements were made and Boehler's angle was measured. In calcaneus measurements, right-left side difference was determined in body height $(\mathrm{BH})$ of the bone $(\mathrm{p}<0.05)$. Boehler's angle was found 20-400, and a significant correlation was found between Boehler's angle and minimum width $(\mathrm{MINW})$ of the bone $(\mathrm{p}<0.01)$. A significant correlation was detected between all other linear bone measurements except for anteroposterior maximum length (MAXL) of calcaneus and width of calcaneal sulcus (WSC) $(\mathrm{p}<0.01)$. By regression of calcaneus measurements, anteroposterior length of the bone can be calculated up to $1 \mathrm{~mm}$. Calcaneus is one of the bones commonly used by anthropologists and forensic scientists as it is preserved well. Morphometric values of calcaneus and the analyses made shall contribute to anatomy science, orthopedic surgery, kinesiology and forensic sciences.

Keywords: Calcaneus, measurement, Boehler's angle, osteology

\section{P-149}

\section{Face transplantation and nervus facialis}

Gorur I, Ozturk L, Boduc E

Department of Anatomy, Faculty of Medicine, Ege University, Izmir, Turkey

Tissue and organ transplantation appeared with the idea of humans that being immortal. One of these composite transplantations is face transplantation. Facial expression muscles need to regain their motor innervations to make these transplantations functional. And this depends on the recovery rate of nervus facialis after the transplantation.

Goal: The goal of this work is to examine these transplantations in terms of basic sciences.

Methods: The two of seven male cadavers which are fixed to $\% 10$ formalin and being in Ege University Anatomy Department are dissected with the methods of arteria temporalis superficialis and aretria facialis pediculus. By the partial dissections in the three cadavers which has no facial skin, the connection of nervus facialis' branching with the facial expression muscles is demonstrated. In the other two cadavers the skin is removed and facial expression muscles are dissected. The information which are derived from the dissected cadavers and from the literature is evaluated together.

Result and Conclusion: These operations have many problems such as scientifically, anatomically, physiologically, psychologically, ethically, technical and economical problems. In this work, the dissections of the flaps of the face which are obtained by imitating the surgical techniques were lasted on the average of 5 hours which is directly proportional with the cadaver studies in the literature. And this is the indicator of the duration of the operations are very long for both patient and the surgent in the sense of the dissections of the flaps and the total period of the operations (such as 15-19 hours). Also postoperative care is very troublesome because the patients have to use immunosupressive medicine for the rest of their lives. And in long term, this causes the side effects that downgrades the life quality of the patients.
Before or after the operation, an exact success percentage about the motor innervation cannot be given yet. Besides, considering the individual variations in facial expression muscles, nervus facialis, arteria and vena facialis, one can say that these operations are 'experimantal operations'.

Keywords: Facial transplantation, nervus facialis, facial expression muscles

\section{P-150}

\section{Evaluation of the upper and lower lip posture in relation with gender in adolescents Class I individuals: a preliminary study}

\author{
Kurkcuoglu $A^{*}$, Karaca $Z^{* *}$, Oguz $\mathrm{O}^{* *}$ \\ *Department of Anatomy, Faculty of Medicine, Başkent University, \\ Ankara, Turkey; ${ }^{*}$ Department of Anatomy, Faculty of Medicine, \\ Cukurova University, Adana, Turkey
}

Objectives: Soft facial tissues as well as lip thickness may show changes basing on age, gender, race and growth and development and the cited issue is important for forensic anthropologists, dentists and plastic surgeons. Forensic anthropologists employ the foregoing information by establishing similarity to facial tissues in determining the identities. Our objective in this study is to evaluate the upper and lower lip thickness in the Turkish race separately in groups consisting of boys and girls and compare the results between the groups and with other races.

Methods: The study was carried out on 80 healthy individuals (40 girls and 40 boys) with Class I skeletal structure (ANB angle of $2^{\circ} \pm 2$ ). The average age of the girls was $23.23 \pm 4.27$ years while the average age of the boys was $24.35 \pm 3.45$ years. The distance of three different points (Ls, Sto, and Li) to line $\mathrm{E}$ (Steiner's aesthetic line) determined on the lateral cephalometric X-ray images of the individuals was measured. These cephalometric images were measured using the software Image-J method in computer. The obtained data were analyzed through statistical methods.

Results: Ls -E, Sto-E and Li -E distances were measured in all cephalometric images. The average value for the Ls-E distance was found as $-11.70 \mathrm{~mm}$ while the minimum value was -26.92 $\mathrm{mm}$ and maximum values: $-1.00 \mathrm{~mm}$; the average value for the Sto-E distance was $-20.73 \mathrm{~mm}$ while the minimum value value was $-37.31 \mathrm{~mm}$ and the maximum value was $-7.59 \mathrm{~mm}$; the average value for the LI-E distance was $-7.17 \mathrm{~mm}$ while the minimum value was $-22.40 \mathrm{~mm}$ and the maximum value was $9.20 \mathrm{~mm}$. The distribution of factors for the measured variable $(p \geq 0.05)$ as a result of the conducted Kolmogrov-Smirnov test was evaluated in accordance with the normal distribution.

Conclusion: Knowing the lip posture provides easiness to orthodontists in planning treatment of patients related to their facial profiles while it provides tips to the forensic anthropologists in the technique of facial reconstruction and to facial cosmetic surgeons in their evaluations as regards the harmony of the bottom part of the face. This study has the nature of a prelimi- 
nary study and we are planning to put forward the gender, and age-related changes that may occur in the upper and lower lip posture in the Turkish race by increasing the number of people.

Keywords: Lip thickness, cephalometry, Class I

\section{P-151}

The beneficial effects of $18 \mathrm{~b}$-glycyrrhetinic acid following oxidative and neuronal damage in brain tissue caused by global cerebral ischemia/ reperfusion in a c57BL/J6 mouse model

Oztanir $\mathrm{MN}^{*}$, Ciftci $\mathrm{O}^{* *}$, Cetin $\mathrm{A}^{* * *}$, Durak MA*, Başak $\mathrm{N}^{* * *}$, Akyuva $\mathrm{Y}^{*}$

${ }^{*}$ Department of Brain and Neurosurgery, Faculty of Medicine, Inonu University, Malatya, Turkey; **Department of Medical Pharmacology,

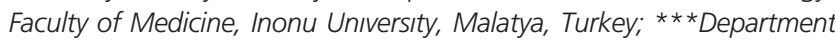
of Histology and Embryology, Faculty Medicine, Inonu University, Malatya; ****Department of Pharmaceutical Toxicology, Faculty Pharmacy, Inonu University, Malatya, Turkey

Objectives: This study investigated the effects of $18 \mathrm{bgly-}$ cyrrhetinic acid (GA) on neuronal damage in brain tissue caused by global cerebral ischemia/reperfusion (I/R) in C57BL/J6 mice.

Methods: In this study we used $40 \mathrm{C} 57 \mathrm{BL} / \mathrm{J} 6$ mice and they were randomly divided into four equal groups $(n=10)$. Shamoperated (SH), I/R, GA (100 mg/kg/day/orally), and I/R+GA. Tissue samples were taken for the determination of biochemical and histological changes at the end of 10th day. Brain samples were processed by routine tissue techniques and embedded in paraffin. 5-İm thick sections of tissues were cut, mounted on slides, stained with Hematoxylin-Eosin (H-E). For immunohistochemical analysis, we used Caspase-3 for determination apoptosis. Sections were exam-ined under a Leica DFC280 light microscope by Leica Q Win and Image Analysis System (Leica Micros Imaging Solutions Ltd.; Cambridge, U.K). Biochemical analysis (TBARS, SOD, CAT, GPx ve GSH) was performed by spectrophotometric methods.

Results: Cerebral I/R significantly induced oxidative stress via an increase in lipid peroxidaitons (decrease TBARS levels) and a decrease in elements of the antioxidant defense systems (CAT, GPx, SOD, GSH).With histopathological evaluation, we detected focal ischemia areas in cerebral cortex, vascular congestion, mononuclear cell infiltration, shrinkage of the cytoplasm, and extensively dark pyknotic nuclei were observed in the neurons. I/R+GA group, histopathological findings were significantly decreased compared with I/R group.

Conclusion: In conclusion, the global I / R causing histological damage and oxidative stress in brain tissue, however GA application eliminates the toxic effects. As a result, GA application were found to be protective against I / R-induced toxic effects.

Keywords: Ischemia-reperfusion, 18b-glycyrrhetinic acid (GA), caspase-3, brain

\section{P-152}

The beneficial effects of chrysin against thioacetamide induced reproductive damage in male rats

Ciftci O*, Cetin $\mathrm{A}^{* *}$, Başak $\mathrm{N}^{* * *}$

*Department of Medical Pharmacology, Faculty of Medicine, Inonu University, Malatya, Turkey; ${ }^{*}$ Department of Histology and Embryology, Faculty of Medicine, Inonu University, Malatya, Turkey; ***Department of Pharmaceutical Toxicology, Faculty of Pharmacy, Inonu University, Malatya, Turkey

The aim of this study is to investigate the beneficial effects of the chrysin supplementation on oxidative stress, sperm characteristics and histological alterations in male reproductive system of rats against thioacetamide (TAA) toxicity. In this study, twenty eight male rats of Wistar Albino origin were randomly divided into 4 groups of 7 as follows: Group 1: Control, Group 2: TAA (200 mg/kg/twice a week), Group 3: Chrysin (50 $\mathrm{mg} / \mathrm{kg}$ ) for 15 consecutive days, Group 4: TAA+Chrysin (200 $\mathrm{mg} / \mathrm{kg} / \mathrm{twice}$ a week $+50 \mathrm{mg} / \mathrm{kg}$ ). Tissue samples were collected on day 15. of TAA treatment. The animals were sacrificed under ether anesthesia and testis tissues were immediately removed. The results showed that TAA caused a significant oxidative damage via induction of lipid peroxidations (elevated TBARS formation) and reduction in the antioxidant defense system potency (decrease SOD, CAT, GSH and GPx levels) in the testis tissue. In addition sperm motility and sperm concentration significantly decreased but abnormal sperm rate and histopathologic testicular damage increased with TAA treatment. Some histopathological changes as loss of maturation in the germinal cells, congestion between seminiferous tubules and arrested cells in division were observed in TAA group. On the other hand, chrysin treatment can prevent oxidative, histopathological and spermatological effects of TAA and can reverse side effects of TAA. Chrysin treatment with TAA may prevent reproductive damages. In the present study, we claim that chrysin supplementation may attenuate the TAA-induced reproductive toxicity in terms of oxidative status of testis, spermatological and histological damage.

Keywords: TAA, chrysin, oxidative stress, testiscular damage, sperm characteristics

\section{P-153}

The protective effects of montelucast on ovary and uterus damage induced by 2,3,7,8-tetrachlorodibenzop-dioxin (TCDD)

Ciftci $\mathrm{O}^{*}$, Cetin $\mathrm{A}^{* *}, \underline{\text { Başak } \mathrm{N}^{* * *}}$

*Department of Medical Pharmacology, Faculty of Medicine, Inonu University, Malatya, Turkey; ${ }^{*}$ Department of Histology and Embryology, Faculty of Medicine, Inonu University, Malatya, Turkey; ***Department of Pharmaceutic Toxicology, Faculty of Pharmacy, Inonu University, Malatya, Turkey

Objectives: In this study, we aimed to investigate the protective effects of montelucast (ML) on ovary and uterus damage induced by 2,3,7,8-tetrachlorodibenzo-p-dioxin (TCDD). 
Methods: In this study, twenty eight female rats (3-4 months) of Wistar Albino origin were randomly divided into 4 groups of 7 as follows: Group 1:Control (Corn oil), Group 2: TCDD (2 $\mu \mathrm{g} / \mathrm{kg} /$ week in corn oil/gavage), Group 3: TCDD + Montelucast ( $2 \mu \mathrm{g} / \mathrm{kg} /$ week in corn oil $+10 \mathrm{mg} / \mathrm{kg} / \mathrm{day}$ ), Group 4: Montelucast group (10 mg/kg/day). The blood and tissue samples were taken from all rats at the end of the experiment for the determination of histological and biochemical changes. Tissue samples were taken for analysis and 5- $\mu \mathrm{m}$ thick sections of tissues stained with Hematoxylen- Eosin staining methods. Sections exam-ined under a Leica DFC280 light microscope by Leica Q Win and Image Analysis System (Leica Micros Imaging Solutions Ltd.; Cambridge, U.K). We determined TBARS, SOD, CAT, GPx and GSH levels as spectrophotometric methods.

Results: The results indicated that ML prevents oxidative damage caused by TCDD via decrease lipid peroxidations (TBARS) and increase antioxidant defense systems (SOD, CAT, GSH, GPx). With histopatological evaluation, uterus and over sections showed normal histological appaerance in control and ML groups. In TCDD group, intense cell infiltration in ovarian tissue and follicular degeneration were observed. In addition to this, we detected significant atretic follicles in the ovary and loss of follicular granulosa cells in TCDD group. We observed epithelium and gland degeneration in uterus in TCDD group. Simple columnar epithelium changed to simple cuboidal epithelim. These findings were significantly decreased in TCDD + ML group.

Keywords: TCDD, ovary, uterus, montelucast, rat

\section{P-154}

\section{Protective effects of apricot on acetaminophene-} induced lung damage

Bayat $\mathrm{N}^{*}$, Yilmaz $\mathrm{I}^{* *}$, Çetin $\mathrm{A}^{* * *}$

*Cengiz Gökçek Gynecologic and Obstetrics Hospital, Gaziantep, Turkey; **Department of Pharmacology, Faculty of Pharmacy, Inonu University, Malatya, Turkey; ***Department of Histology and Embryology, Faculty of Medicine, Inonu University, Malatya, Turkey

Objectives: Acetaminophen ( APAP), chemically named Nacetyl-p-aminophenol, is a widely used pain reliever and fever reducer. Apricot is a fruit which has a high content of carotenoids, $\mathrm{C}$ and $\mathrm{E}$ vitamins and selenium. This study was planned to observe the protective effects of apricot (10\% apricot) on acetaminophen induced lung damage.

Methods: In this study, twenty four Sprauge-Dawley rats were randomly divided into four equal groups. Groups: 1.Group $(\mathrm{n}=6)$ : Control, 2.Group $(\mathrm{n}=6)$ : Acetaminophene (APAP) group (835 mg/kg single dose, orally), 3.Group (n=6): APAP (835mg/kg single dose, oralyl) $+10 \%$ Apricot, 4 . Group (n=6): 10\% Apricot. At the end of the experiment, rats were sacrified under ketamine/xylazine anesthesia. For light microscopic evaluation, lung samples were fixed in $10 \%$ formalin. The lung samples were processed by routine tissue techniques and were embedded in paraffin. Paraffin-embedded specimens were cut into $5 \mathrm{~mm}$ thick sections, mounted on slides and stained with HematoxylenEosin (H-E). Sections exam-ined under a Leica DFC280 light microscope by Leica Q Win and Image Analysis System (Leica Micros Imaging Solutions Ltd.; Cambridge, U.K).

Results: In control and $10 \%$ Apricot groups, lung tissue observed normal histopathological appaerence. In APAP group, alveolar wall thickness, mononuclear cell infiltration, vascular congestion, hemorrhage, enlargement of alveoli were detected. In APAP $+10 \%$ group, all these histopathological findings were decreased significantly.

Conclusion: We observed that $10 \%$ apricot has protective effects on acetaminophen induced lung damage.

Keywords: Acetaminophen, apricot, lung, histopathological damage

\section{P-155}

The ratio of the length of extremity to length of body in basketball players: a comparative study.

Cetin $\mathrm{A}^{*}$, Korkmaz MF**, Sarıkaya E***, Altıkulaç E***, Çiçek $\mathrm{H}^{* * *}$, Çöken $\mathrm{E}^{* * *}$, Avc1 $\mathrm{H}^{* * *}$, Sezgin $\mathrm{K}^{* * *}$

*Department of Anatomy, Faculty of Medicine, Inonu University, Malatya, Turkey; **Department of Orthopedics and Traumatology, Faculty of Medicine, Inonu University, Malatya, Turkey; ***Period II Student, Faculty of Medicine, Inonu University, Malatya, Turkey

We aimed to compare the ratio of the length of extremity to length of body in young individuals who play basketball and the ones who do not. When we compare the length of body/forearm ratio; those who play basketball was 6.71 and for the ones who do not play was 7:09. Accordingly; forearm length was significant in basketball players (p:0.001). While height / (hand length) average ratio was 9.06 for basketball players, it was 10.0 for the ones who do not play basketball. Hand length was also increased in basketball players (p:0.001). While height / (lower extremity length) average ratio was 1.73 for basketball players, it was 1.86 for the ones who do not play basketball. Lower extremity length was also increased in basketball players (p:0.004). Height/(thigh length) average ratio was 1.73 for basketball players, it was 1.86 for the ones who do not play basketball. Thigh length was also increased in basketball players (p:0.002). Height / (leg length) average ratio was 3.51 for basketball players, it was 3.86 for the ones who do not play basketball. Leg length was also increased in basketball players (p:0.0001).

\section{P-156}

\section{Evaluation of abdominal hysterectomy in a} private hospital

Beytur L,$\stackrel{* *}{-}$, Köse $\mathrm{E}^{* *}$, Özbağ D**

*Department of Gynecology and Obstetric, Private Malatya Hospital, Malatya, Turkey; ${ }^{*}$ Department of Anatomy, Faculty of Medicine, Inonu University, Malatya, Turkey 
Objectives: Hysterectomy is called to removal of the uterus by abdominal, vaginal or laparoscopy. Hysterectomy can be done benign or malignant reasons. In this study, the abdominal hysterectomy for benign pathology such as abnormal uterine bleeding, uterine myoma, postmenopausal bleeding, uterine prolapse, benign ovarian cysts, endometrial hyperplasia, endometrial polyps were evaluated.polyps were evaluated.

Methods: The data of patients files with hysterectomy since 2010 were screened retrospectively in Gynecology and Obstetrics outpatient clinic. The vaginal hysterectomy due to uterine prolapse were excluded from the study. Demographic data, operative time, postoperative complications, pathology findings were evaluated.

Results: The mean age of the 37 patients was 52.4 (41-64) year. In $21(56.76 \%)$ of these patients uterine myoma, in 6 patients $(16.22 \%)$ dysfunctional uterine bleeding, in 5 patients $(13.52 \%)$ endometrial hyperplasia, in 2 patients $(5.40 \%)$ adenomyosis, in 2 patients $(5.40 \%)$ postmenopausal bleeding, and in one patient $(2.70 \%)$ the endometrial polyps were present. Abdominal hysterectomy was performed classic phannenstiel incision. The average operation time was 65 minutes (55-90). None of the patients had drains inserted. The mean of hospitalisation time was 3.2 (2-5) days. One patient had postoperative blood transfusion. No complications were reported in other patients.

Conclusion: Hysterectomy procedure, when done with appropriate indications and surgical techniques is a process that is less risk of complications, safe and successful procedure. A rapid improvement in symptoms after the hysterectomy, the patient's quality of life resolves and becomes.

Keywords: Uterus, hysterectomy, abdominal hysterectomy

\section{P-157}

\section{Non invasive method in the treatment of kidney stones placed at lower calyx: retrograde intrarenal surgery}

Beytur A*, Köse E ${ }^{* *}$, Oğuz F*,Çakmak BS*, Topçu I*, Güneş A* *Department of Urology, Faculty of Medicine Inonu University, Malatya, Turkey; **Department of Anatomy, Faculty of Medicine Inonu University, Malatya, Turkey

Objectives: Kidney stone formation is a relatively common condition. In the treatment of the stone, protective measures should be taken primarily, if stone is composed, firstly crushing is done with sound waves from outside the body, when it does not crush the stone, surgery is performed. Previously, open surgery and minimally invasive methods were in use, now stones achieved by entering into the body in a natural way, and the stones are cleaned as a result of technological developments. Stone localization is also important in the treatment of kidney stones. The most difficult ones to treat are stones located to the lower calyx of the kidney. In this study, retrograde intrarenal surgery (RIRS), which is being implemented in our clinic, success was evaluated in the treatment of the lower calyx stones.
Methods: Ten patients were evaluated retrospectively who underwent RIRS due to kidney stone in lower calyx in last 1 year.

Results: There is not any pediatric patient in the study group. The mean age of the patients was $51.6(28-81)$ years. There are three male and seven patients female. The average size of stone was calculated $1.45(1-2) \mathrm{cm}$. In one patient, there was a stone too in the upper calyx as well as in the lower calyx Stones and had cleaned in the same operation. The avaradge hospitalitation time was calculated as 1.1 days (1-2). One patient had residual stone and then the process was repeated. None of the patients had any complications.

Conclusion: Urinary stone disease prevalence is $2-3 \%$. Some of the factors affecting the formation of urinary stone disease are; genetics, gender, age, geography, climate, age, occupation, obesity, fluid intake etc. There is still not any drug prevents the formation of stones in the urinary tract. RIRS is safe to treat kidney stones in the lower calyx.

Keywords: Kidney kidney stone, lithotripsy

\section{P-158}

\section{Variation of the axillary artery}

Cetin A, Çay $M$

Department of Anatomy, Faculty of Medicine, Inönü University, Malatya, Turkey

Arteria axillaris variation was confronted in a 40-years-old male cadaver during the axillary region dissection performed with an evidence-based student group in the anatomy laboratory. The arteria axillaris yielded three branches of the same level at 31 $\mathrm{mm}$ distal from the first costa at the right extremity. The first branch (arteria thoracoacromialis) had a diameter of $4 \mathrm{~mm}$ and divided into two after proceeding $1 \mathrm{~cm}$. While one of the branches moved towards the thorax, the other the deltoid muscle. The second branch (arteria thoracica superior) had a diameter of $3 \mathrm{~mm}$ and went towards the thorax. The third branch (arteria thoracica lateralis) had a diameter of $2 \mathrm{~mm}$ and made towards the thorax lateral wall. The arteria axillaris yielded arteria subscapularis having a diameter of $7 \mathrm{~mm}$ towards the medial at $73 \mathrm{~mm}$ distal from the first costa. The arteria axillaris yielded arteria axillaris at $25 \mathrm{~mm}$ distal from the first costa at the left extremity. In addition, it yielded the arteria thoracoacromialis having a diameter of $3 \mathrm{~mm}$. This branch proceeded towards the neck side. At the same point, the arteria axillaris yielded the arteria thoracica lateralis having a diameter of 2 $\mathrm{mm}$ towards the medial. It yielded another branch having a diameter of $1.7 \mathrm{~mm}$ at the mid-point of the arteria thoracoacromialis and the arteria thoracica lateralis. This branch made towards the arm. This branch was found to be variation. No arteria thoracica superior was observed on the left side. The arteria axillaris yielded a branch having a diameter of 2 $\mathrm{mm}$ at $15 \mathrm{~mm}$ distal from the point of emergence of the arteria thoracoacromialis. This branch made towards the spina scapula. This branch was found to be a variation, too.

Keywords: Axillary artery, variation, cadaver 


\section{P-159}

A study of personal, social and university compliance between Turkish nationals from abroad and foreign students

Özdemir $F^{*}$, Onay I**, Uzun A*, Emirzeoğlu $M^{*}$, Altunsoy E* Nazari B*

*Department of Anatomy, Faculty of Medicine, Ondokuz Mayıs University, Samsun, Turkey; **Department of Primary Education, Faculty of Educaiton, Ondokuz Mayıs University, Samsun, Turkey; $* * *$ Faculty of Medicine, Ondokuz Mayıs University, Samsun, Turkey

The aim of the present study is to describe and compare between the Turkish Nationals from abroad and foreign students in Ondokuz Mayis University, faculty of medicine, about personal, social compliance and facilities of university. The questionnaire had 45 questions and 5 options were used in the present study. The present study included 32 Turkish and 33 foreign students, who were selected through purposive sampling. In data analysis, the questionnaire items were divided into three groups as personal, social and university compliance; independent samples t-test and Pearson's correlation analysis was performed, $\mathrm{p}$ value at or less than 0.05 was accepted as statistically differences. Our result revealed that the Turkish students reported more positive answers of personal compliance questions than the foreign group $(\mathrm{p}<0.05)$. There were significant correlation between the personal category and social categories $(\mathrm{p}<0.05, \mathrm{r}=0.57)$; also between social category and university category $(\mathrm{p}<0.05, \mathrm{r}=0.49)$. The conclusion of the present study, there was no significant differences between the Turkish students and the foreign students about social and university facilities; however about personal adaptation is better in the Turkish students.

Keywords: Turkish national, foreign national, student, medical education, social compliance, personal compliance

\section{P-160}

\section{Early complications of thyroidectomy}

Karataş $T^{*}, *$, Özbağ D**, Çay $M^{* *}$, Şenol D**, Korkmaz $\mathrm{MF}^{*}$,***

*Department of General Surgery, Malatya State Hospital, Malatya, Turkey; **Department of Anatomy, Faculty of Medicine, Inönü University, Malatya, Turkey; $* * \star$ Department of Orthopedics and Traumatology, Faculty of Medicine, Inönü University, Malatya, Turkey

Objectives: Our purpose is to present complications in early postoperative period in 308 cases who underwent thyroidectomy.

Methods: Between March 2003 and March 2013 in Malatya State Hospital, General Surgery Clinic, files and operative notes of 308 patients who underwent thyroidectomy were analysed retrospectively. Gender, age, preoperative diagnosis, primary or recurrent cases, the type of surgery (bilateral subtotal-BST, bilateral total-BTT, unilateral subtotal-UST and unilateral total thyroidectomy-UTT) and early postoperative complications (hypocalcemia, bleeding, hoarseness, infection and seroma) were recorded.

Results: 287 (93.18\%) female and 21 (6.81\%) male are of total 308 patient who underwent thyroidectomy. Age range is of 16 to 75 . Median age is 40.78. $283(91.88 \%)$ multinodular, 18 $(5.84 \%)$ nodular goiter and $7(2.27 \%)$ thyroid cancer were operated. There are $301(97.72 \%)$ primary and $7(2.28 \%)$ recurrent patient. 180 (58.44\%) BTT, 100 (32.46\%) BST, 7 (2.27\%) UST and 4 (1.29\%) UTT were performed. There was seen complications in $20(6.49 \%)$ cases who underwent BTT (19) and BST (1). A complication in the BST was only the bleeding. $10(3.24 \%)$ hypocalcemia (8 primary and 2 recurrent cases), 4 (1.29\%) bleeding, 2 (0.649\%) hoarseness (1 primary and 1 recurrent case), 2 (0.649\%) infection and $2(0.649 \%)$ seroma were observed.

Conclusion: Thyroidectomy was performed mostly in females. The most common type of surgery is BTT. The second is BST. The most frequent complication is hypocalcemia. Bleeding is in second. Hoarseness, seroma and infection are following them. Most of the complications were observed in patients who had BTT. Hoarseness and hypocalcemia rate was higher in recurrent cases who underwent BTT. In thyroid surgery, it should be more careful when it is performed BTT in recurrent cases.

Keywords: Goiter, thyroid surgery, complication

\section{P-161}

\section{Protective effects of apricot on acetaminophene- induced acute heart damage in rats}

$\underline{\text { Yilmaz I* }}$, Çetin A**, Gül S**

*Department of Pharmacology, Faculty of Pharmacy, Inonu University Malatya, Turkey; **Department of Histology and Embryology, Faculty of Medicine, Inonu University, Malatya, Turkey

Objectives: This study was planned to observe the protective effects of \% 10 sun-dried organic apricot (SDOA) supplementation on $835 \mathrm{mg} / \mathrm{kg}$ oral acetaminophen (APAP) induced acute heart tissue damage in rats.

Methods: In this study, twenty four Sprauge-Dawley rats were randomly divided into four equal $(n=6)$ groups. Groups: 1 . Group $(n=6)$ : Control (Rats were not given any toxicological or pharmacological active material, chove and water just were given ad libitum. 2. Group $(n=6)$ : Acetaminophene (APAP) group (835 $\mathrm{mg} / \mathrm{kg}$ single dose orally, chove and water just were given ad libitum.). 3. Group ( $n=6)$ : APAP+ $10 \%$ sun-dried organic apricot supplemented diet: $(835 \mathrm{mg} / \mathrm{kg}$ orally single dose acetaminophen +during 45 days $10 \%$ rate sun-dried organic apricot supplemented diet and water were given ad libitum.). 4. Group ( $\mathrm{n}=6): 10 \%$ sun-dried organic apricot (during 45 days $10 \%$ rate apricot supplemented diet and water were given ad libitum.). At the end of the study (on 45th day), rats were sacrified under ketamine/ xylazine anesthesia. Heart tissue samples were taken for biochemical and histological investigaton. For light microscopic evaluation. For light microscopic evaluation, heart tissue samples 
were processed by routine tissue techniques and were embedded in paraffin. Tissue samples were cut into $5 \mathrm{~mm}$ thick sections, mounted on slides and stained with Hematoxylen- Eosin (H-E). Sections exam-ined under a Leica DFC280 light microscope by Leica Q Win and Image Analysis System (Leica Micros Imaging Solutions Ltd. Cambridge, U.K).

Results: In control and 10\% sun-dried organic apricot groups, heart tissue showed normal histological appearance. Hovewer, APAP treated rats showed some histological changes such as eosinophilic cytoplasm and pyknotic nuclei cells, mononuclear cell infiltration, intracytoplasmic vacuolization, congestion, necrosis and hemorrhage. 10\% sun-dried organic apricot supplementation significantly reduced APAP-induced histopathological changes. Within serum biochemical parameters (AST, ALT, ALP and Totally biluribin), only third group of ALP parameter indicated statistically significant difference than others.

Conclusion: We observed that, $10 \%$ sun-dried organic apricot has protective effects on acetaminophen-induced heart damage in rats.

Keywords: Acetaminophen, apricot, heart, rat

\section{P-162}

Protective effects of apricot on acetaminopheneinduced acute kidney damage in rats

Y1lmaz I*, Çetin $\mathrm{A}^{* *}$, Şahin $\mathrm{H}^{* *}$

*Department of Pharmacology, Faculty of Pharmacy, Inonu University Malatya, Turkey; *Department of Histology and Embryology, Faculty of Medicine, Inonu University Malatya, Turkey

Objectives: This study was planned to observe the protective effects of $10 \%$ sun-dried organic apricot supplementation on $835 \mathrm{mg} / \mathrm{kg}$ oral acetaminophen (APAP) induced acute kidney tissue damage in rats.

Methods: In this study, twenty four Sprauge-Dawley rats were randomly divided into four equal (n=6/group) groups. Groups: 1 . Group ( $\mathrm{n}=6)$ : Control (Rats were not given any toxicological or pharmacological active material, chove and water just were given ad libitum. 2.Group ( $\mathrm{n}=6)$ : APAP group $(835 \mathrm{mg} / \mathrm{kg}$ single dose oral chove and water just were given ad libitum.). 3. Group $(n=6)$ : APAP $+10 \%$ sun dried organic apricot: $(835 \mathrm{mg} / \mathrm{kg}$ orally single dose acetaminophen +during 45 days $10 \%$ sun dried organic apricot supplemented diet and water were given ad libitum. 4 . Group ( $n=6$ ): $10 \%$ sun dried organic apricot (during 45 days $10 \%$ sun dried organic apricot supplemented diet and water were given ad libitum.). At the end of the study (on 45th day), rats were sacrified under ketamine/ xylazine anesthesia. Kidney tissue samples were taken for biochemical and histological investigaton. For light microscopic evaluation, kidney tissue samples were processed by routine tissue techniques and were embedded in paraffin. Tissue samples were cut into $5 \mathrm{~mm}$ thick sections, mounted on slides and stained with Hematoxylen- Eosin (H-E). Sections exam-ined under a Leica DFC280 light microscope by Leica Q Win and Image Analysis System (Leica Micros Imaging Solutions Ltd. Cambridge, U.K).
Results: In control and 10\% sun dried organic apricot groups, kidney tissue showed normal histological structure. The glomerulus, proximal and distal tubules are in normal histological appearance. In APAP group, glomerular and tubular distortion and degeneration, interstitial hemorrhage between tubules, vascular congestion, mononuclear cell infiltration, tubular dilatation and oedema, narrowing of Bowman's space were determined. Moreover within vacuolar degeneration of the proximal and distal tubules as a result of foamy appearance and hydropic changes were observed. Within serum biochemical parameters (AST, ALT, ALP and Totally biluribin), only third group of ALP parameter indicated statistically significant difference than others.

Conclusion: We observed that sun dried organic apricot has protective effects on acetaminophen-induced renal damage in rats.

Keywords: Acetaminophen, apricot, kidney damage, rat.

\section{P-163}

The protective role of chrysin on harmful effects of formaldehyde on the development of hippocampus in rat fetuses; a histopathologic study

Cuglan $\mathrm{S}^{*}$, Yildiz A**, Irmak Sapmaz $\mathrm{H}^{*}$,Bakirci $\mathrm{S}^{* * *}$, Kose $\mathrm{E}^{*}$, Ekinci $\mathrm{N}^{* * * *}$

*Department of Anatomy, Faculty of Medicine, Inonu University, Malatya, Turkey; **Department of Histology and Embryology, Faculty of Medicine, Inonu University, Malatya, Turkey; $* * *$ Department of Anatomy, Faculty of Medicine, Duzce University, Duzce, Turkey; $* * * *$ Department of Anatomy, Faculty of Medicine, Sakarya University, Sakarya, Turkey

Objectives: In this study, we aimed to determine possible harmful effects of formaldehyde exposure on the hippocampus of fetuses. In addition, potential protective role of chrysin $(\mathrm{CH})$ against these effects.

Methods: 58 female rats were divided into 6 groups and were tried to become pregnant. 10 rats which were not pregnant excluded from the study. It was administered every other day during 7-20 days of pregnancy to group I (Control) intraperitoneally (i.p.) saline, group II (CH) by gavage $20 \mathrm{mg} / \mathrm{kg} \mathrm{CH}$, group III (FA-0,1) i.p. 0,1 mg/kg FA, group IV (FA-1) i.p. 1 $\mathrm{mg} / \mathrm{kg} \mathrm{FA}$, group $\mathrm{V}(\mathrm{FA}-0,1+\mathrm{CH})$ i.p. $0,1 \mathrm{mg} / \mathrm{kg} \mathrm{FA}$ and by gavage $20 \mathrm{mg} / \mathrm{kg} \mathrm{CH}$ and group VI (FA-1+CH) i.p. $1 \mathrm{mg} / \mathrm{kg}$ $\mathrm{FA}$ and by gavage $20 \mathrm{mg} / \mathrm{kg} \mathrm{CH}$. $\mathrm{CH}$ was applied one day before FA application. Fetuses obtained by caesarean section. Histological staining was performed to determine the impact on the hippocampus development in fetuses. Stained preparations were examined with a study microscope (Leica DFC280). Statistical analysis was performed.

Results: In the pyramidal layer of the FA groups section was observed mild decrease in neuronal density, neuronal shrinkage and condensation of the nucleus. These observed changes were prominent in the group of FA-1. Histopathological injury of the FA-1 group significantly higher than the control group $(\mathrm{p}<0.05)$. Some sections in the FA groups were observed apoptotic cells in the pyramidal layer. Injury scores in the groups 
treated FA with $\mathrm{CH}$ decreased compared to the group treated with only FA. However, this reduction was not found to be statistically significant $(\mathrm{p}>0.05)$.

Conclusion: It was determined that low dose FA exposure leads to histological damages on the development of the hippocampus of the rat fetuses. Although these effects were prevented by $\mathrm{CH}$, these changes were not statistically significant.

Keywords: Formaldehyde, chrysin, rat fetus, hippocampus

\section{P-164}

Two lobule spleen - A case report

Uğuz Gençer $\mathrm{C}^{*}$, Koşar MI*, Tetiker $\mathrm{H}^{*}$, Balcı $\mathrm{Y}^{* *}$

*Department of Anatomy, Faculty of Medicine, Muğla Sıtkı Koçman University, Muğla, Turkey; **Department of Forensic Medicine, Faculty of Medicine, Muğla Sıtkı Koçman University, Muğla, Turkey

Spleen is an intraperitoneal organ located in the left upper quadrant between fundus of stomach and diaphragm. The largest lymphoid organ, spleen can display various congenital anomalies. The anomalies vary in many types such as persistent lobulation, multilobulated spleen, accessory spleen, wandering spleen, ectopic spleen, asplenia and polysplenia which can be associated with a syndrome. The other organ anomalies may or may not be present with spleen anomalies. In cases with spleen anomalies when the other organ anomalies are not present have no clinical symptoms. These variants are incidentally recognized in the routine examinations, during autopsy and disection. Present study is a case which was observed during routine autopsy in the department of forensic medicine. Two lobule spleen was observed in the normal anatomic position of a 50 year-old male's cadaver. Both lobes have one common hilum and covered by one capsule. In this cadaver heart anomalies and other accompanying organ anomalies were not observed. Awareness of these variants is important in clinical conditions that require splenectomy such as torsion/ infarction or hematologic disorders. Even a small part of the remaing spleen can take over function fully therefore both lobules should be resected. In conclusion, to be aware of anatomic variations and anomalies is important for surgeons and radiologists to avoid diagnostic misinterpretations and treatment.

Keywords: Spleen, congenital anomalies, anatomy, autopsy

\section{P-165}

Anatomy education in complementary medicine

Bozer C

Department of Anatomy, Faculty of Medicine, Trakya University, Edirne, Turkey

Since 1990's The World Health Organization (WHO) has supported Traditional and Complementary Medicine in terms of practice, research primarily in the universities. People in developed countries have preferred natural therapy methods due to adverse effects of drugs used in modern medicine and people have preferred natural therapy methods due to financial inadequacy in developing countries. Also the use of traditional and complementary therapies in medicine has been supported in terms of public health. Like in other countries, traditional and complementary therapies in medicine have gained importance in our country due to its wide health benefits, safety and minimal side-effects compared to chemical agents, in recent years. There were institutions offering courses in these therapy methods in Turkey. Recently, the Ministry of Health constituted the Department of Traditional, Complementary and Alternative Therapies in Medicine for determining the traditional, complementary and alternative therapies, organizing the courses corresponding with these therapy methods, standardization of the education levels of the practitioners and determining the ethical issues and legal liabilities. The therapy methods regulated by the Ministry of Health are; acupuncture, apitherapy, phytotherapy, hypnosis, hirudotherapy, homeopathy, chiropractic, cupping, maggot therapy, mesotherapy, ozone therapy, prolotherapy, reflexology and ostheopathy. Furthermore, there are other therapy methods like neural therapy, bioresonance and kinesiology those have not been regulated by the Ministry of Health yet. In our country, some private and state universities have constituted research, practice and education centers in different titles for the traditional, complementary and alternative therapies in medicine. Currently, a proposal by the Ministry of Health includes the constitution of a national organization with the title; the Institute of Traditional and Complementary Therapies in Medicine. The anatomy education takes an important part in the courses of these therapy methods. New and different fields of education and employment are developing for the anatomists in our country in the courses of these therapy methods.

Keywords: Complementary medicine, anatomy, education 